\title{
THE EDUCATIONAL NEEDS OF HOME-BASED STROKE PATIENTS AND FAMILY CAREGIVERS IN MALAYSIA
}

by

Che Rabiaah Mohamed

A thesis submitted to the Victoria University of Wellington in fulfilment of the requirements for the degree of

Doctor of Philosophy in Nursing

Victoria University of Wellington 


\begin{abstract}
Many research studies have been done in relation to the educational needs of patients and families post-stroke based in Western countries but limited studies have been conducted in non-Western countries. This study explored the educational needs of home-based stroke patients (HBSP) and family caregivers in the Kelantan state located at the northern part of Peninsular Malaysia. The other areas of focus were to identify the resources available for HBSP and their families, the practice of health professionals in relation to the provision of information and education and the early rehabilitation needs for HBSP and family caregivers. A further aim was to develop education strategies that will help patients and their families to minimise risk of complication post-stroke and support the advancement of their quality of life. This study used a qualitative approach guided by a theoretical framework "Stroke Care Community Model (SCCM)" developed for this research. This study was in two phases. Phase 1 involved semi-structured interviews with three kinds of participants: stroke patients $(n=5)$, family caregivers $(n=5)$ and key members from health professional groups $(n=12)$. Phase 1 established the educational activities currently received and needed by patients and families. Phase 2 involved presenting the findings from Phase 1 to the previously interviewed health professionals through focus groups and individual interviews with the aim of establishing priorities and processes to develop education strategies for HBSP and their families. The findings revealed that the provision of information and education does not meet the needs of many patients and families. There is no national or local programme provided to address patients' and families' needs. The educational needs are on a wide range of topics including the nature and the impacts of stroke, how and why stroke happens, how to prevent stroke recurring, prevention of complications and promoting faster recovery. The need for skills on managing patients at home include positioning, strengthening, mobilisation, prevention of complications, feeding, swallowing, managing co-morbid conditions, basic exercise and safety. The findings also revealed some needs are religiously and culturally bound around prayer and traditional healthcare practice such as urutan tradisional (traditional massage). Prayer and reciting Holy Qur'an are part of cognitive training, coping strategies, rehabilitation therapy and a source of psychological support. Therefore, there is a need to take the knowledge of what makes a difference to
\end{abstract}


health outcomes of patients and families in a western context and work with this, utilising what is important in the culture of people at a local level. The next step to progress addressing educational needs requires a structured approach involving the formation of a multi-disciplinary stroke team that incorporates cultural and religious practice.

Keywords; stroke, home-based stroke patients (HBSP), Malaysian stroke, family caregivers, informal caregivers, urutan tradisional (traditional massage), traditional healthcare practice, thematic and content analysis, 


\section{Acknowledgements}

First of all, I wish to express my gratitude to my first supervisor, Dr. Katherine Nelson for her many valuable comments and suggestions, all of which have greatly improved my thesis. I am fortunate indeed to have her as my main supervisor. She has always been accessible, effective, and understanding. Dr. Nelson's consistent guidance, encouragement and support throughout my study have enabled me to complete this doctoral work.

I am also grateful to my second supervisor Associate Professor Pamela Wood for her valuable comments, suggestions and support throughout my study especially in the later part of this course. Her contribution was indeed invaluable. Profound thanks to Associate Professor Cheryle Moss for providing the relevant support and guidance required for this work in the early part of my study. I am indeed grateful for her insights into the various issues concerning my study.

I would also like to thank University Sains Malaysia for providing funded study leave throughout the course of my study. A very special thanks to former dean of School of Health Science, USM, Professor Zainul Fadziruddin Zainuddin for his support for my application to undertake this study leave and throughout the course of study. I would also like to thank Professor Ahmad Zakaria, Dean of the School of Health Science for his support for an extension of my study leave enabling me to complete my study.

Profound thanks to all the funding agencies (1) The Malay Chair Grant, (2) New Zealand Postgraduate Study Abroad Awards and (3) Victoria PhD Submission Scholarship which provided funding toward the completion of my study.

It is my pleasure to acknowledge all the participants: stroke patients, family caregivers, health professionals from Hospital Universiti Sains Malaysia, academic nursing staff and representative from National Stroke Association of Malaysia who cooperated so willingly to make this research possible. 
My eternal gratitude to my lovely husband Abdullah for your love, appreciation, support, and encouragement and patience throughout the course of my study. A special thank you for allowing me to be alone in New Zealand at the end of my study. A special thank you for the sacrifices you made in taking three years unpaid leave to accompany me and our children to New Zealand. To all my children, Ira, Ummi, Wani, Afiq, Alia and Aleef, a special thank you for your love, understanding, and constant support and encouragement throughout this journey. Finally, my special gratitude to my parent, other family members and all relatives and friends in Malaysia.

Terima Kasih (Thank you) 


\section{Table of contents}

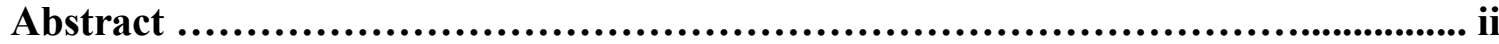

Acknowledgements ................................................................................ iv

Table of contents ......................................................................... vi

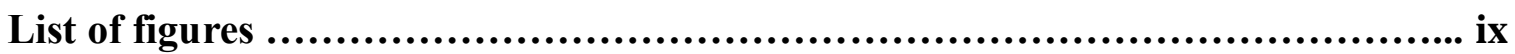

List of tables ............................................................................... ix

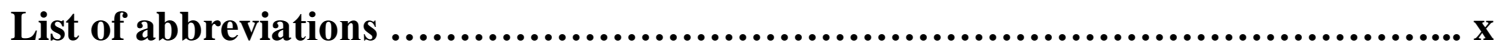

Chapter 1: Introduction to the research .......................................... 1

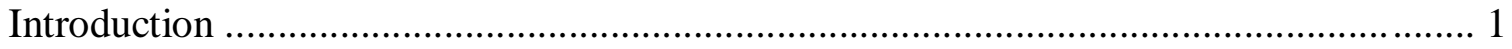

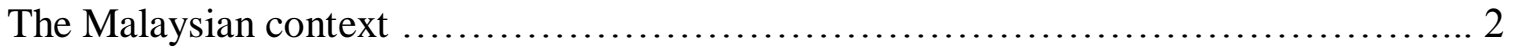

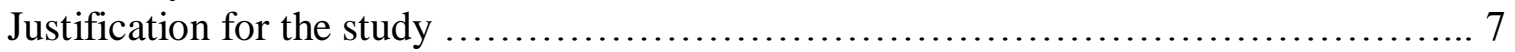

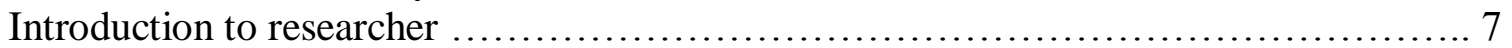

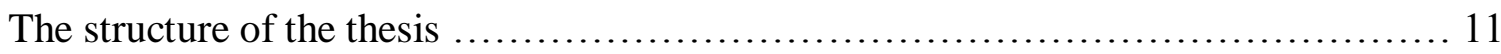

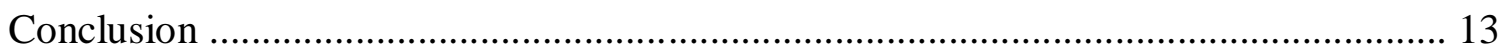

Chapter 2: Malaysia health care system ......................................... 14

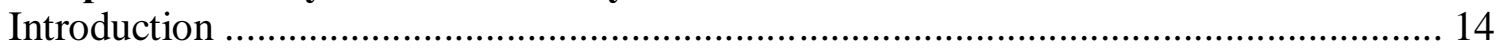

General overview of Malaysian health care system .............................. 14

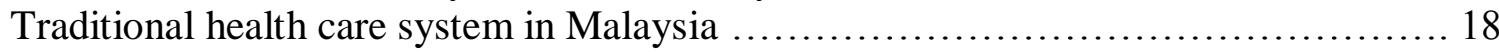

Healthcare services related to stroke patients in Malaysia ............................ 20

The family caregivers or informal healthcare providers of stroke patients in Malaysia . 24

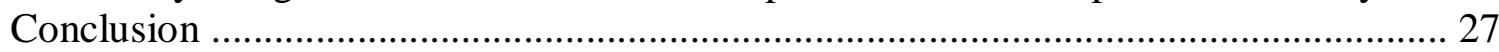

Chapter 3: Literature review ...................................................... 30

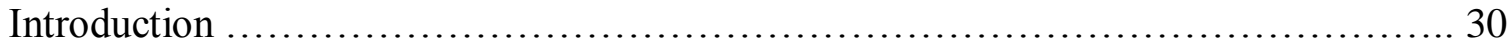

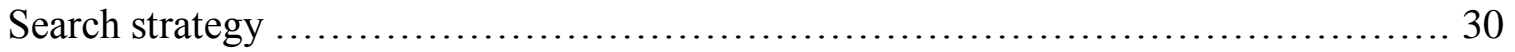

The nature of stroke and stroke statistics ...................................... 32

Stroke patients' health journey: The experience of the impact of stroke ............... 36

The physical effects ...................................................................... 41

The psychological effects .......................................................... 43

The psychosocial effects ........................................................... 45

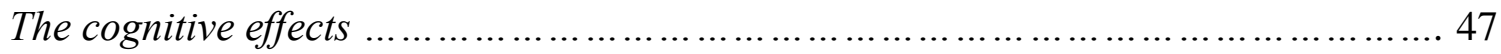

The impacts of caring for a loved one who has experienced a stroke ........................ 47

The needs of stroke patients and family caregivers .............................. 52

Rehabilitation needs of stroke patients .................................................... 52

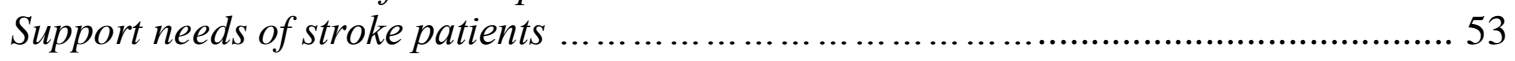

Support needs of caregivers ................................................................................. 55

Studies on the information and education needs of stroke patients and caregivers ......... 57

The information and education needs of the caregivers ................................................ 57

The information and education needs of patients and the caregivers ........................... 60

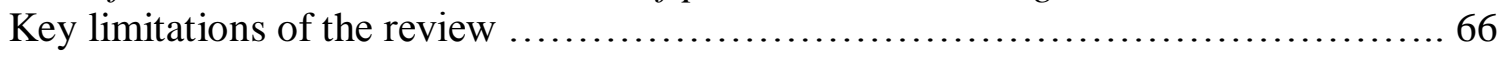

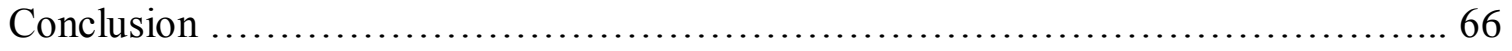


Chapter 4: Theoretical/conceptual framework .....................................68 68

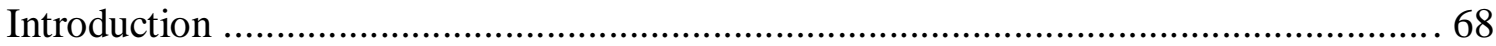

The Wagner's Chronic Care Model (The Wagner's CCM) ........................... 68

The Theoretical Model (SCCM) .................................................. 70

Components of the Stroke Care Community Model (SCCM) ......................... 72

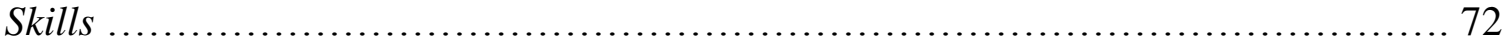

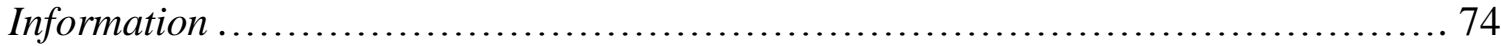

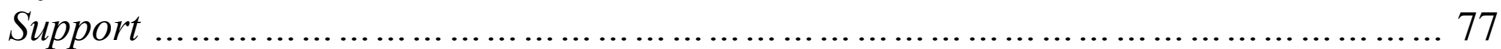

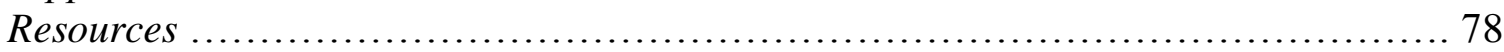

Interface and relationship with the community ................................... 78

Interface and relationship with the healthcare service ........................... 79

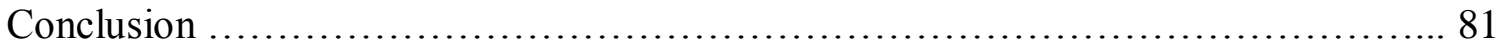

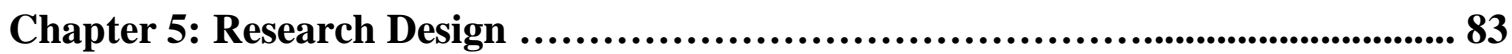

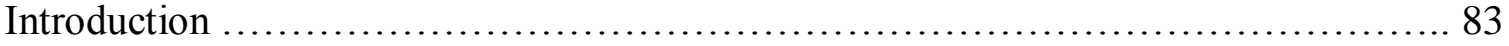

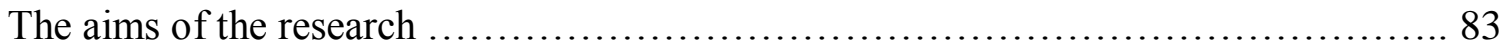

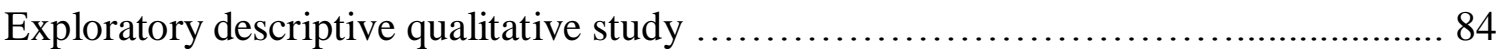

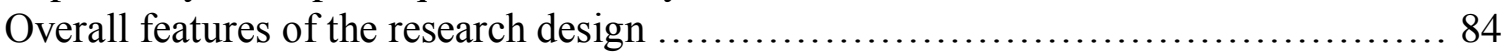

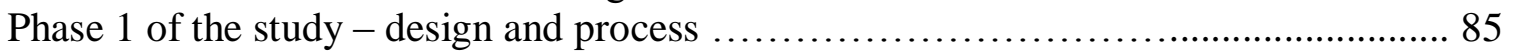

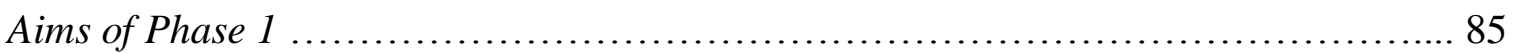

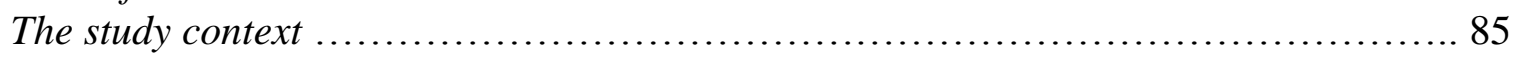

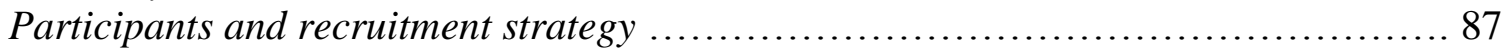

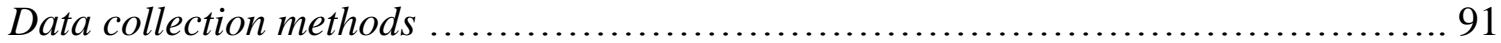

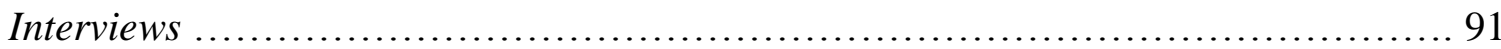

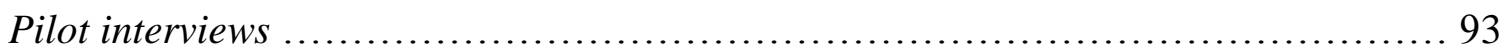

Interviews with stroke patients and family caregivers ................................ 93

Interviews with health professionals ............................................... 94

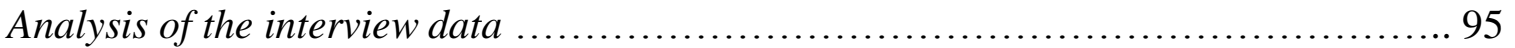

Ethical consideration in Phase 1..................................................... 98

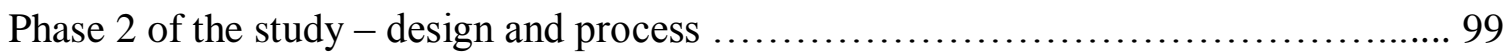

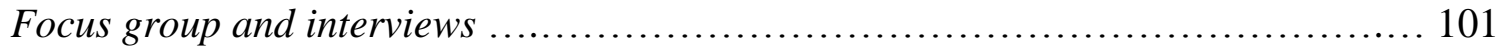

Researcher as moderator and facilitator ............................................ 103

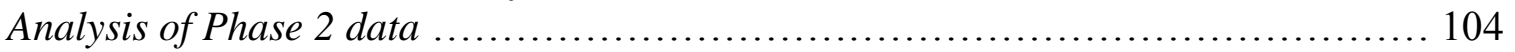

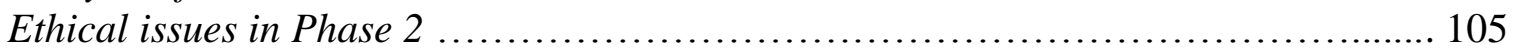

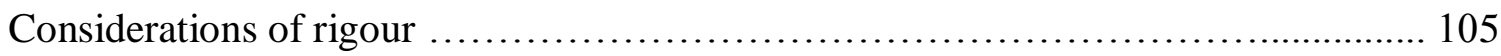

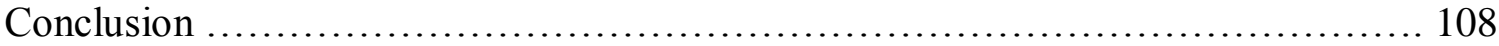

Chapter 6: Process and findings of the analysis of stroke patients and family caregivers ........................................................................ 109

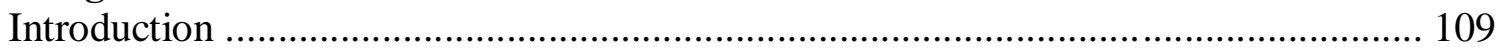

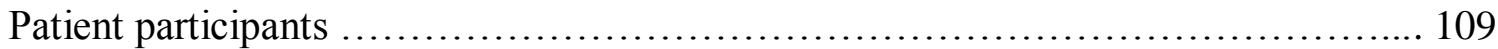

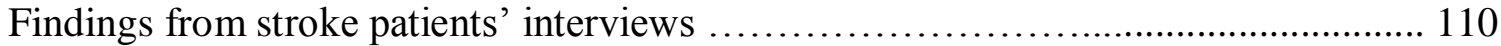

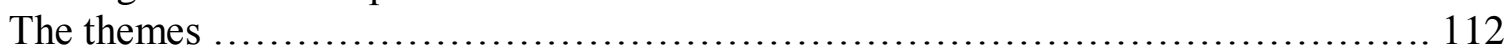

Findings of the caregivers' interviews ........................................... 122

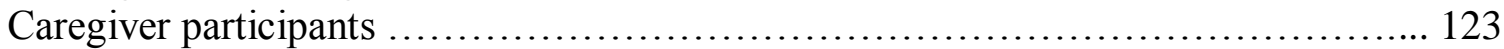

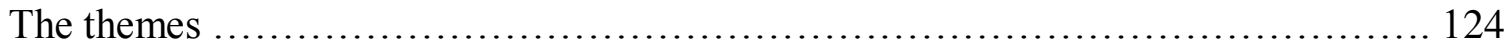


Conclusion .................................................................. 134

Chapter 7: Process and findings of the analysis of health professionals .......... 136

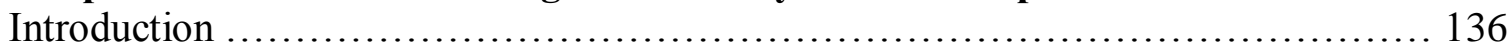

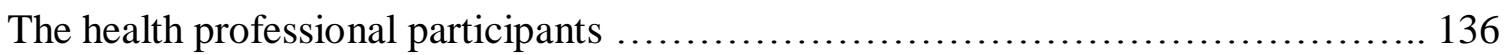

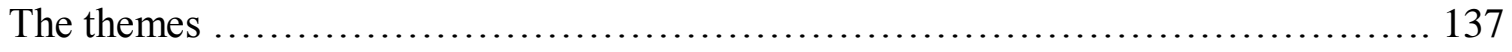

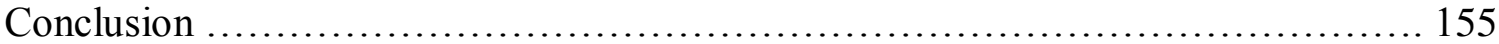

Chapter 8: The educational needs identified in the interview with stroke patients,

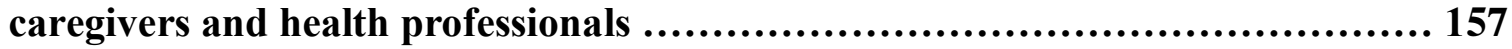

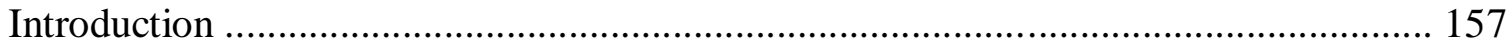

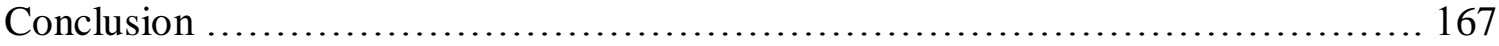

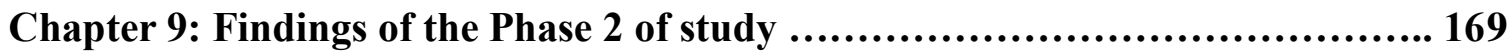

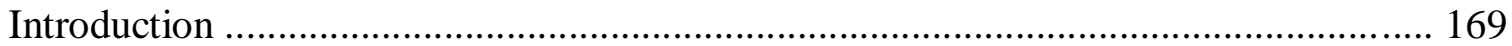

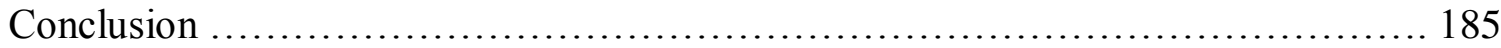

Chapter 10: Discussions and implications ........................................... 186

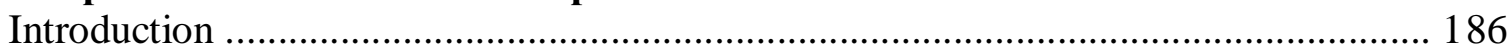

Reflection of the research .................................................. 186

Discussion of the main findings ................................................ 190

Implications for practice, policy and further research ........................... 209

Further research ..................................................... 211

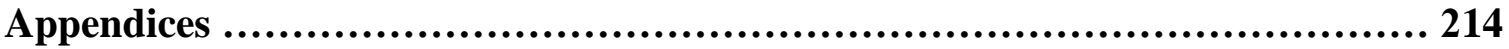

Appendix 1: Example of interview guide used for stroke patients and family

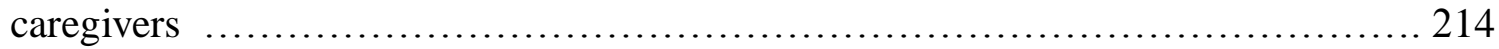

Appendix 2: Example of interview guide used for health professionals ............... 218

Appendix 3: Example of interview guide used for NASAM member ............... 222

Appendix 4: Ethics approval from Victoria University of Wellington Human Ethics

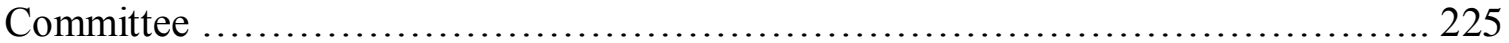

Appendix 5: Letter to Director of HUSM ..................................... 226

Appendix 6: Response from Director of HUSM .............................. 228

Appendix 7: Information sheet and consent for interview for Phase 1of study ........ 229

Appendix 8: Letter and flyer requesting volunteer from NASAM be interviewed ..... 232

Appendix 9: Introductory letter from supervisor ............................... 234

Appendix 10: Information sheet and consent for Phase 2 of study ................. 235

Appendix 11: Interview guide for focus group and individual interview for Phase 2 of

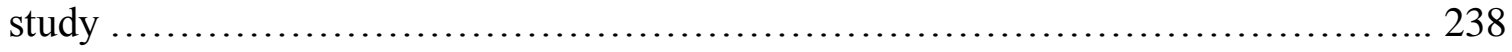

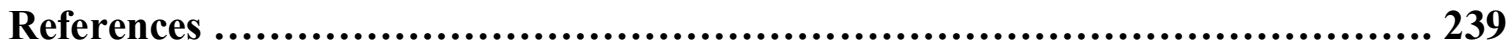




\section{List of figures}

Figure 1: Map of the state of Kelantan in the East Coast of Peninsular Malaysia ....... 2

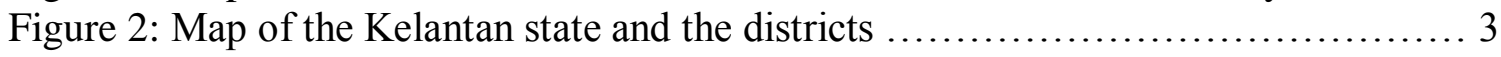

Figure 3: Healthcare delivery system in Malaysia $\mathrm{MOH}$........................... 16

Figure 4: Flow chart for patient with suspected stroke ........................... 21

Figure 5: The Stroke Care Community Model (SCCM) ........................ 71

\section{List of tables}

Table 1: Health facilities in Malaysia Health Care System .......................... 18

Table 2: Socio-demographic characteristics of interviewed stroke patients ........... 110

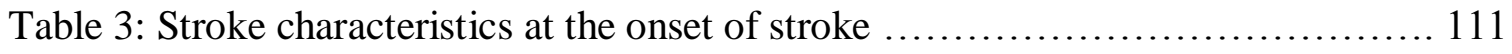

Table 4: Socio-demographics features of family caregivers ........................ 123

Table 5: Socio-demographics of the 12 health professional participants ............... 137

Table 6: Summary of aspects of education and information needs of stroke patients and

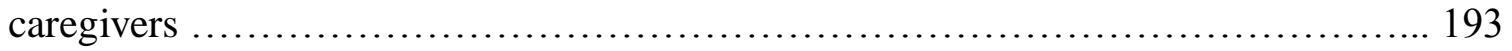




\section{List of abbreviations}

$\begin{array}{ll}\text { ADL } & \text { Activities of Daily Living } \\ \text { ASEAN } & \text { Association of Southeast Asian Nations } \\ \text { Wagner's CCM } & \text { Wagner's Chronic Care Model } \\ \text { CES-D } & \text { Scale - Center for Epidemiologic Studies Depression Scale } \\ \text { CPG } & \text { Clinical Practice Guideline } \\ \text { CPSP } & \text { Central post-stroke pain } \\ \text { CVA } & \text { Cerebrovascular accident } \\ \text { DM } & \text { Diabetes mellitus } \\ \text { GP } & \text { General Practitioner } \\ \text { HBSP } & \text { Home-based stroke patient } \\ \text { HUSM } & \text { Hospital Universiti Sains Malaysia } \\ \text { MDST } & \text { Multidisciplinary Stroke Team } \\ \text { MOH } & \text { Ministry of Health } \\ \text { NASAM } & \text { National Stroke Association of Malaysia } \\ \text { NGO } & \text { Non-government organisation } \\ \text { NIHM } & \text { National Institutes of Health, Malaysia } \\ \text { PSP } & \text { Primary support person } \\ \text { QOL } & \text { Quality of life } \\ \text { RCT } & \text { Randomized controlled trial } \\ \text { SCCM } & \text { Stroke Care Community Model } \\ \text { SF-36 } & \text { Short Form Questionnaires 36 } \\ \text { TIA } & \text { Transient ischemic attack } \\ \text { USA } & \text { United States of America } \\ \text { USM } & \text { Universiti Sains Malaysia } \\ \text { VUW } & \text { Victoria University of Wellington } \\ \text { WHO } & \text { World Health Organisation } \\ \text { 9MP } & \text { Ninth Malaysian Plan } \\ & \end{array}$




\section{Chapter 1: Introduction to the research}

\section{Introduction}

Stroke or cerebrovascular accident (CVA) or strok or angin ahmar in Malay language is one of the major burdens to the Malaysian healthcare system due to the increase in the number of stroke victims. The Disease Burden Study indicated that stroke is ranked third behind ischemic heart disease and mental illness (Ministry of Health $[\mathrm{MOH}], 2005$ ). Stroke is also the leading cause of severe disability in Malaysia which is similar to other developing and developed countries (National Stroke Association of Malaysia [NASAM], 2007). Statistics from National Stroke Association of Malaysia (NASAM) show that approximately 40,000 Malaysian people are disabled by stroke every year. The number of stroke patients admitted to the Malaysian Ministry of Health $(\mathrm{MOH})$ hospitals has been increasing $(\mathrm{MOH}, 2006)$ from 13,868 in 2000 to 16,805 in 2004 (National Institutes of Health, Malaysia [NIHM], 2006). For those who survive a stroke, the effects are usually life long, and maximising recovery requires rehabilitation.

Due to limited comprehensive rehabilitation services and shorter length of hospital stay, many more patients require rehabilitation and continuity of care at home. Therefore the responsibilities of caring and rehabilitation are shifted to the family members. Given the increased role of families in post-stroke care, it is important to understand what preparation would be important for them to take on this role. This thesis focuses on the stroke patient and their family caregivers in Malaysia. This study will use the term 'stroke patient' throughout to refer to people who suffered a stroke. Some disability advocates challenge terminology that positions the disease before the person (Auslander \& Gold, 1999), however an enormous amount of published literatures related to stroke were found using this term. Even it is also the common usage in Malaysia. In addition, the term stroke is widely used compared to CVA among the public and health professionals. Family caregivers are family members who provide care for other family member following stroke. The thesis presents the findings of a study which explored the educational needs of home-based stroke patients (HBSP) and family caregivers in the Kelantan state located at the northern part of Peninsular Malaysia. The other important area of focus for the research were the resources available for 
HBSP and their families, the practice of health professionals in relation to the provision of information and education and the early rehabilitation needs post-stroke.

This chapter commences with a brief introduction to stroke and stroke care in Malaysia. This is followed by the aims of the study and a brief overview of the people involved. The Malaysian context of the study is presented. The chapter concludes with an introduction to the researcher followed by an overview of the structure of the thesis.

\section{The Malaysian context}

Malaysia is located in Southeast Asia. It is bordered by Thailand in the north and the island nation of Singapore in the south. Malaysia comprises West Malaysia (Peninsular Malaysia) and East Malaysia (Sabah and Sarawak). East Malaysia, located on the island of Borneo, is separated from Peninsular Malaysia by the South China Sea with a distance 540 kilometres. Malaysia consists of a federation of 13 states and the federal territories of Kuala Lumpur, Labuan and PutraJaya (Figure 1).

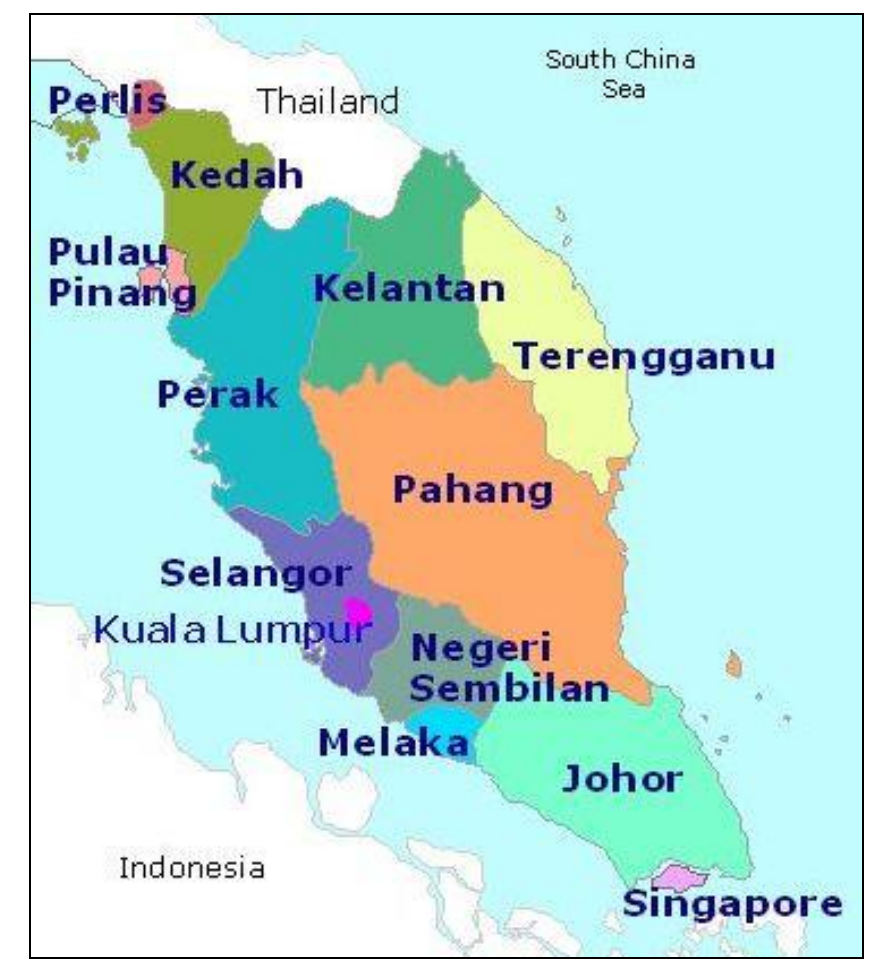

Figure 1. Map of the state of Kelantan in the East Coast of Peninsular Malaysia

Source: http://www.mymalaysiabooks.com/.../Malaysia_states.htm, reproduced with permission 
Malaysia has a multiethnic population of 27.7 million consisting of $65 \%$ Bumiputera (Malays and ethnic minorities), 26.1\% Chinese and 7.6\% Indian. Kelantan where this study was conducted is located in the north-eastern corner of the Malaysia Peninsula and shares a border with Thailand in the north. Kelantan has a population of about 1.4 million; $95 \%$ are Malay with Chinese, Indian and Orang Asli (indigenous people) making up the rest. Figure 1 shows the location of Kelantan in Peninsular Malaysia and Figure 2 is a detailed map of Kelantan.

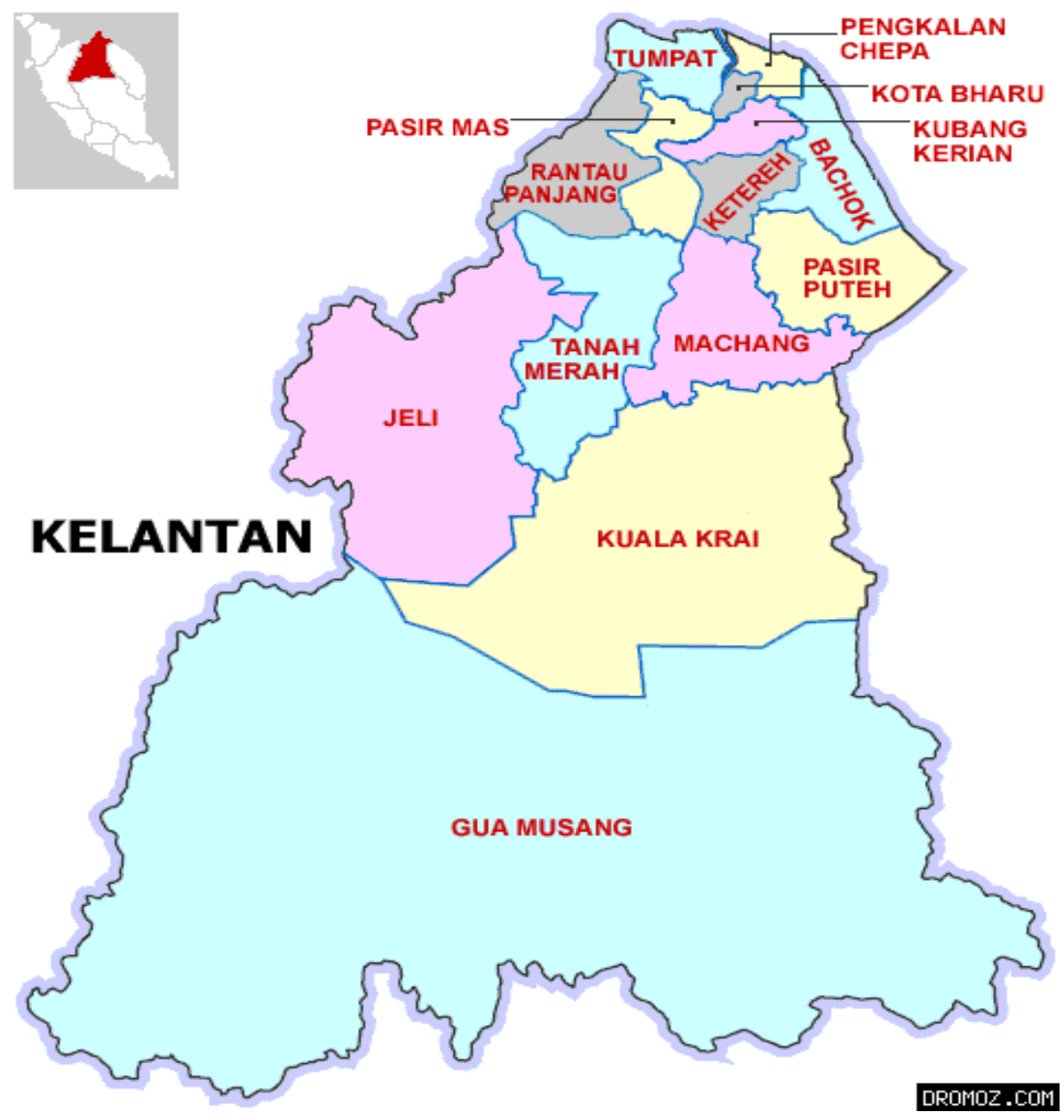

Figure 2. Map of the Kelantan state and the districts

Source: http://www.dromoz.com/image/sketch/sk4488e05094bfe034689094.png, reproduced with permission.

Kota Bharu is the largest town and the administrative capital of the Kelantan state. Currently there are 10 government hospitals in Kelantan. Of these, eight are district 
hospitals, one a state general hospital and the other a university teaching hospital. Two of the hospitals also function as tertiary referral centres for people with stroke. These two hospitals are located in the Kota Bharu district. Each district shown in Figure 2 may refer stroke patients for admission to these two hospitals.

Healthcare in Malaysia is provided by the public and private sectors and non-governmental organisations (NGOs) (Merican \& Yon, 2002). The public (government) and private healthcare services work as two parallel systems and are complemented by nongovernmental organisations and traditional and complementary medicine (T/CM). The public healthcare service which is under the $\mathrm{MOH}$ is the main healthcare provider in the country. Public health services are provided by general hospitals, district hospitals, polyclinics, health centres, rural clinics, midwives clinics and mobile clinics. "Although primary care was provided both by the public and private sectors, the rural areas were almost entirely served by a wide network of government health clinics and hospitals rapidly developed in the 1960s" (Leng \& Barraclough, 2007, p.1). The state general hospitals are considered as secondary referrals which receive all the referred cases from district hospitals. Moreover, Kuala Lumpur General Hospital which is situated in the capital city, Kuala Lumpur is known as a tertiary referral centre for all 13 states general hospitals throughout Malaysia (Poi, Forsyth \& Chan, 2004). Apart from that, some of the states have University Teaching hospitals. There are three teaching hospitals in Malaysia, which are attached to public universities: Hospital University Sains Malaysia (HUSM) in Kelantan, Hospital University Kebangsaan Malaysia and University Malaya Medical Centre at the capital city of Kuala Lumpur. These hospitals serve as teaching centres to those particular universities. My study site is at HUSM which is one of the teaching hospitals and also a tertiary referral centre.

To date, there are 130 government hospitals throughout Malaysia (MOH, 2008). The focus of healthcare delivery in these hospitals is on the provision of secondary and tertiary medical services (MOH, 2006). In line with the global focus on traditional and complementary medicine (T/CM), Malaysia developed a national policy on T/CM in 1999. Some of the traditional healthcare practices include traditional healers such as dukun or bomoh and sinseh 
as well as traditional healing systems such as homeopathy and naturopathy (Nik Rosnah, 2005). Currently there is an increasing focus on the role of T/CM globally and particularly in Malaysia. One of the important traditional healthcare practices or therapies is urutan tradisional. Urutan tradisional is widely used among Malaysians to treat people with paresis or paralysis. It is also used for sprain, muscle pain, numbness and to promote uterine contraction during puerperium. Given that Malaysia utilises a range of traditional health practices, this thesis includes details of these in relation to stroke rehabilitation. Further details of the Malaysian health service in relation to stroke care are described in Chapter 2.

Stroke care is one of the major issues in Malaysia and in particular within the study setting (NIHM, 2006). The effects of stroke cause sudden life changes to most patients and their families. Literature revealed that once stroke happens, patients need to seek immediate medical attention to minimise the consequences of stroke (Adams, 1998; Lott, Hennes \& Dick, 1999). However, the context of my setting is different from what has been stated in the literature. Some patients do not seek timely treatment. This might be due to the lack of knowledge of the importance of timely treatment to reduce the consequences of stroke. With limited comprehensive rehabilitation service and shorter length of stay, patients are discharged early to the care of their families (NIHM, 2006). Therefore families play an important role in providing care and rehabilitation for patients at home. Studies based on Western context revealed that stroke patients and families need information and education on many aspects of stroke and stroke care (Eames, McKenna, Worral \& Reed, 2003; Knight, Worral \& Ross, 2006; Wiles, Pain, Buckland \& McLellan, 1998). Due to a lack of research on this area in Malaysia, we do not know how stroke patients and their families manage in the community. Therefore there is an area of concern regarding the educational needs of Malaysian stroke patients and their family caregivers post-stroke. Research is needed to examine what educational activities are currently received and needed. Most of the published literature was from Western countries where the context is very different from Malaysia as a non-Western country in terms of cultural practice, religious belief and ethnicity as well as rehabilitation practice post-stroke. 
This research focuses on educational needs post-stroke. Many studies have been undertaken to examine and explore the needs of patients and families post stroke in particular their educational needs (Anderson et al., 2000; Brereton \& Nolan, 2002; O'Connell et al., 2003; Wiles et al., 1998). However, these studies were undertaken among patients and families from backgrounds which are very different from my setting. Tan et al. (2006) indicated the need for public education on many aspects of stroke as one of the important areas of research in Asia. Even in developed countries, it has been demonstrated that post-stroke care is problematic (Gupta \& Thomas, 2002; Samsa et al., 1997). My study is an important study as there is a gap in knowledge in how to effectively minimise complications and maximise quality of life post discharge from acute stroke in the absence of a formalised rehabilitation system, as is the situation in much of Malaysia. Therefore this study was purposely designed to elicit the educational needs of Malaysian stroke patients and families with particular reference to Kelantan which is less developed compared with other states in Malaysia.

There is also a gap in knowledge on what are the available resources and support (in the hospital or community) for stroke patients and caregivers in Malaysia and whether stroke family caregivers are adequately prepared for the care giving role. This clearly indicates a study needed to be carried out in relation to the needs of the family caregivers and how they cope with their roles in caring for stroke patients in Malaysia as this might be different from other countries such as the UK, the United States (US), Australia and New Zealand. Different coping mechanisms may be practised by stroke patients and their family in Malaysia compared with other countries. This might be due to differences in cultural practices, religious beliefs, ethnic back grounds, living arrangements, socioeconomic status or different support services provided by the government.

The current Malaysian population of approximately 27.7 million (MOH, 2008) also raises an issue of the distribution of resources for stroke care within the states and the regions. There is an inequitable distribution of resources in stroke care within the public sector in Malaysia (Tan et al., 2006). For example, $80 \%$ of the neurologists are located in the capital city of Kuala Lumpur with a population of about a fifth of Malaysia's total population. Due to the 
complexity of managing patients post-stroke, examining patients and families at home is critical.

\section{Justification for the study}

As research on the educational needs of stroke patients and families is based on the context of Western countries, this research will provide an insight into the needs of stroke patients and families in a setting where rehabilitation is minimal. Given the current situation in Malaysia related to stroke and post-stroke care, researching the educational needs of stroke patients and their families is an important contribution to stroke care in Malaysia. In addition, the findings will contribute to the international knowledge on stroke care where the practices are culturally and religiously bound. As a nurse I also expect that this study will contribute to the body of nursing knowledge and give great benefit and value to customer service, health care providers and policy makers in Malaysia. The findings of the study on the educational need for stroke patients and family caregivers in Malaysia could promote evidence-based practice. It is hope that in the future, it will provide a positive challenge for Malaysian community healthcare services to develop education resources to satisfy the demand for knowledge and encourage nurses to care for stroke patients. More significantly, it should greatly benefit patients by identifying areas where risk of complication post-stroke can be minimised and support the advancement of their quality of life. Indeed, this could then lead to a reducing the burden to the Malaysian healthcare system by facilitating recovery and rehabilitation.

\section{Introduction to researcher}

I was motivated to carry out this study due to my clinical experience as a nurse, as a community member, as a nurse educator and my previous research. In my daily life and working experience for more than 25 years as well as research experience, I have had a lot of exposure to neurological patients in particular stroke patients. Most stroke patients in my work settings were discharged home early, irrespective of the level of disability from stroke. Patients and families were not adequately prepared for the sudden life changes after stroke. This might be due to the lack of established policy related to providing information and education to patients and families in the acute setting prior to discharge and in the home 
setting. There is also a question whether patients and families have enough education resources to manage at home. Furthermore, from my daily experience dealing with patients and families in the community, I found that patients and family caregivers had a lot of misunderstanding about stroke and lacked the knowledge and skills of home-management of stroke. However, no studies have been conducted to examine this issue. This situation stimulated my interest in stroke.

Apart from my working experiences as a nurse and as a member in the community, my career as a nurse educator further developed my interest in stroke. By having appropriate academic and professional qualifications, I was involved in teaching diploma and undergraduate nursing programmes at the School of Health Science, University Sains Malaysia (USM). Besides other nursing courses, the majority of course subjects that I taught were related to neurological nursing where stroke is a major component of the course. From this teaching and supervision experience, I gained more knowledge related to stroke. This also led to more exposure to patients and family in the hospital setting and drove me to examine their needs. Experience and knowledge obtained by undertaking an advanced course in "Neurosurgery Nursing" at USM provided some more insights on the various problems faced by patients with neurological disorders. This course is supported by the International Development Programme (IDP), Australia, whereby some of the course content was taught by a lecturer from IDP. The theoretical and practical knowledge gained during this course especially in relation to illness or injury to the brain was very challenging, interesting and useful.

In addition to my personal and working experience related to stroke patients, this research is also a continuation of my Master degree studies (Mohamed, 1998) which mainly focused on communication difficulties faced by neurological patients. The interest, knowledge and experience gained during the advanced course in "Neurosurgery Nursing" are among the contributing factors to the decision to undertake research related to aphasic stroke patients for my Masters thesis. The experience in searching literature and conducting interviews with patients served as a strong foundation for my present study. This was the beginning of the idea to undertake this doctoral thesis. 
Furthermore, the most wonderful and useful experience throughout my working experience is dealing with stroke patients, families and health professionals with different ethnic backgrounds, religious practices and cultural beliefs in Western countries. In 1997, I accompanied my family to England so that my husband could pursue his doctoral studies. It was a great opportunity to have working experience for nearly four years in setting which was different from my own. My daily involvement with stroke patients at the in-patient rehabilitation unit in Birmingham provided some great experiences and exposure to the services and support received by stroke patients in a different setting. The daily involvement of rehabilitation therapists such as speech-language pathologists, physiotherapists and occupational therapists and nurses in the in-patient stroke rehabilitation setting impressed me. Observing the daily support received by patients in terms of personal care, mobilising, feeding and socialising provided some insight to the daily needs of patients post-stroke. Other positive experiences related to the provision of information and support received prior to discharge home, including the assessment of the suitability of the house and the support for the transition from hospital to community setting.

From these experiences, the marked differences with the Malaysian setting are in terms of the involvement of the families in the acute and post-acute setting and the support received at home. In my setting, some families play a significant role in assisting patients from the acute setting through the rehabilitation period at home. Some of them even assist health professionals in providing care for their stroke family member in the hospital. This could be due to the different cultural practice between the two settings.

Such experiences were very useful and led me to think about the strategies to improve poststroke care in Malaysia. Knowing the resources in terms of support, knowledge and skills received by patients and families in a setting other than Malaysia, led to my interest in examining the resources currently received and needed by patients and families in Malaysia. Given the lack of comprehensive rehabilitation services in Malaysia for stroke patients and their caregivers, research is needed to examine the education and support needs of these two groups. Are they fully educated and prepared prior to discharge as well as being supported 
while at home? Do they receive similar resources to those stated in the literature? Are patients given assistance and support to reintegrate or socialise into the community?

To date, little is known about the needs of stroke patients and their families in the Malaysian context. Therefore it is an important area of study to explore the educational need of stroke patients and family caregivers post-stroke. More significantly, the area of study is considered to have an important impact on the nation's future. I am particularly interested to develop the strategies or method to improve post-stroke care in Malaysia. The study therefore identified and explored the educational needs of Malaysian home-based stroke patients (HBSP) and their family caregivers based on their perspectives and those of the healthcare professionals, and how these needs could be addressed.

The main objectives of this study were to:

1. Identify the educational needs of stroke patients and family caregivers through their perspectives and those of healthcare professionals;

2. Identify current practices of healthcare professionals in relation to the education and health information they provide for stroke patients and caregivers in preparation for discharge;

3. Identify the early rehabilitation practice of home-based stroke patients and their caregivers;

4. Establish what information and education or resources are presently available that people can access; and

5. Prioritise and develop implementation plans for the development of health education resources that extend existing practice, minimise complication post-stroke and maximise quality of life of stroke patients and caregivers in the home.

In order to achieve the research objectives, my research was designed so that people who were directly involved in the care of people with stroke as well as patients and families at home were interviewed to obtain their views on the issues of the enquiry. The nature of stroke care, recovery and rehabilitation involves the multidisciplinary team. Given the devastating effects caused by stroke and the nature of functional disabilities faced by the 
stroke patients, there is a crucial need for the involvement of families in home-care. Therefore five stroke patients, five family caregivers and 12 multidisciplinary health professionals who are the key people in stroke care were interviewed.

\section{The structure of the thesis}

The thesis is presented in 10 chapters. This chapter has provided an introduction to the topic and background of the study regarding the educational needs of Malaysian stroke patients and their families post-stroke. It also presents an overview about the stroke patients and their family caregivers in the Malaysian context. The Malaysian health care system that involved the modern and traditional healthcare practices in relation to the stroke services and care was also highlighted in brief. This chapter also provided some researcher background related to working experiences and qualifications that led to the interest to undertaken this research. The aims of the study, the justification of the study, the research objectives and other elements of the study were presented to give an overview of the study.

Chapter 2 provides an overview of the Malaysian healthcare services in general. This chapter describes the main healthcare providers in the country: public health services, private healthcare services as well as complementary healthcare services, nongovernmental organisations (NGOs) and traditional and complementary medicine (T/CM). The chapter also provides an overview of the role of traditional massage in post-stroke care in Malaysia.

Chapter 3 presents a review of the relevant literature. This chapter commences with the literature search strategy followed by the overview of the concept of stroke and stroke statistics world-wide. The chapter describes the health journey of stroke patients and family caregivers including the impact of stroke on patients and their family caregivers. It provides important knowledge and insight into the health needs of patients and their caregivers poststroke and is used as a basis for the present study. Then, it is followed by the studies of the educational needs of stroke patients and caregivers following stroke. The appraisal of the literature reveals a gap in the knowledge on how to maximise stroke recovery and the quality of life of the stroke patients and family caregivers and minimise complications post stroke. 
This chapter concludes with the key limitations of the literature and the areas needed for this study.

Chapter 4 introduces the study framework of the "Stroke Care Community Model" (SCCM) which served as a guide for this study. The development of this was informed by Wagner's Chronic Care Model (Wagner's CCM). The framework provides an overview of the importance of informing and preparing patients and families in stroke rehabilitation. The four components of SCCM are described and the educational needs of patients and families on those four elements provided.

Chapter 5 describes and discusses the methodology and two-phase research design of the study. The application of the research design and processes of each phase are explained in detail focusing on the aims of each phase, participant and recruitment strategy, methods of data collection and data analysis. The chapter describes the steps taken to ensure the validity and reliability of the data collected. Lastly, the chapter provides the information on the ethical issues encountered in the study and how these have been dealt with.

Chapter 6 presents the process and findings of the interviews with the first two groups of participants in Phase 1 of the study- stroke patients and family caregivers. The chapter commences with the general background of the patients and caregivers. It is followed by the stroke characteristics of the interviewed patients. The findings from the stroke patient and caregiver interviews are presented separately. The details of the findings are described under the thematic headings.

Chapter 7 presents the findings of the 12 health professional interviews from Phase 1 of the study who was working in the hospital, academic nursing staff and with a key member from the National Stroke Association of Malaysia (NASAM). The findings are reported under several thematic headings. The analysis commences with the general background of the study participants. The chapter ends with the summary of the findings from the health professional interviews. 
While Chapters 6 and 7 provide the separate findings of the three groups of the study participants from Phase 1 of study, Chapter 8 provides the integrated analysis of the findings from those three groups of participants. This analysis was done at the completion of Phase 1 of the research, and was used to inform the basis of the discussion in Phase 2. The integrated analysis is mainly focus on identifying the educational needs of the patients and family caregivers post-stroke. The findings are presented under nine headings.

Chapter 9 presents the process and findings of the focus group and individual interviews from the Phase 2 of the study. The participants were from those health professionals interviewed in the Phase 1. This chapter provides the findings on the health professionals' views related to the processes and priorities of developing and implementing educational strategies for home-based stroke patients (HBSP) and their family caregivers as identified in Phase 1 the study. This is followed by a synthesis of the whole findings.

Chapter 10 presents the implications of the theoretical model, reflection on the research process and the implications and plans related to addressing the education needs. Then, it is followed by a discussion, suggestions and recommendations and conclusions. The discussion is presented in relation to the findings of the literature review, findings from the field study and methodology. The recommendations are based on the cultural and societal values of the Malaysian stroke patients and their caregivers. It also presents the implications of the practice, policy and further research.

\section{Conclusion}

This chapter has demonstrated a brief overview of stroke, stroke care and healthcare practice in Malaysia. Stroke is found as one of the major burdens to the Malaysian healthcare system and the leading cause of severe disability. Given the current situation in Malaysia related to stroke and stroke care coupled with the increased roles and responsibilities of families in post-stroke care, researching patients and families need specifically their educational needs is an important contribution to stroke care to maximise rehabilitation Malaysia. The next chapter will focus on the Malaysian 
healthcare system and the provision of healthcare services related to stroke and the role of traditional massage in post-stroke care. 


\section{Chapter 2: Malaysian Healthcare System}

\section{Introduction}

This chapter presents an overview of the Malaysian healthcare system. It describes the main healthcare providers in the country: public health services, private healthcare services and complementary health services, non-governmental organisations (NGOs) and traditional and complementary medicine (T/CM). It also describes the provision of healthcare services related to stroke and the role of traditional massage in post-stroke care.

\section{General overview of Malaysian health care system}

Malaysia has one of the best healthcare systems in the region and Malaysian health status has improved significantly since independence in 1957 (Ministry of Health [MOH], 2005). The public health services are based on a system devised by the MOH to provide access to basic health services to all people irrespective of their religious belief, socioeconomic status or ethnic background (MOH, 2006). Besides NGOs and T/CM, public health services are complemented by other ministries and government agencies. These include the Ministry of Education, Ministry of Defence and Ministry of Home Affairs. Teaching hospitals, school health services and supplementary feeding programmes in rural schools are under the Ministry of Education. The Ministry of Defence is responsible for the provision of healthcare services for military personnel and the Ministry of Home Affairs is in charge of the non-Malay indigenous groups which are collectively called Orang Asli (aborigines) (Nik Rosnah, 2005).

The MOH healthcare system encompasses all aspects of care such as promotive, preventive, curative and rehabilitative services (Hanafiah, 1996; Nik Rosnah, 2005). This is to ensure the provision of high quality health service to achieve excellent health

status for the population. Public healthcare is financed by general taxation, fee-forservice for private healthcare, and an employer-financed health benefit scheme. Health services in the rural areas are provided by mobile teams and health centres as well as being supported by secondary referral state general hospitals which are situated in every 
state. Public healthcare is highly subsidised by government and consumers are charged a minimum or no fee when they access primary, secondary and tertiary healthcare facilities (Nik Rosnah, 2005). It is common knowledge among Malaysians that the costs to access public health services are lower than the private health service. A great deal of effort has been implemented to keep the cost of healthcare low while ensuring the services are efficient. In addition, the government (public) health service also provides free healthcare for school-age children, government staff and pensioners (Hanafiah, 1996; Leng \& Barraclough, 2007). Figure 3 shows the layers of the healthcare delivery system in Malaysia.

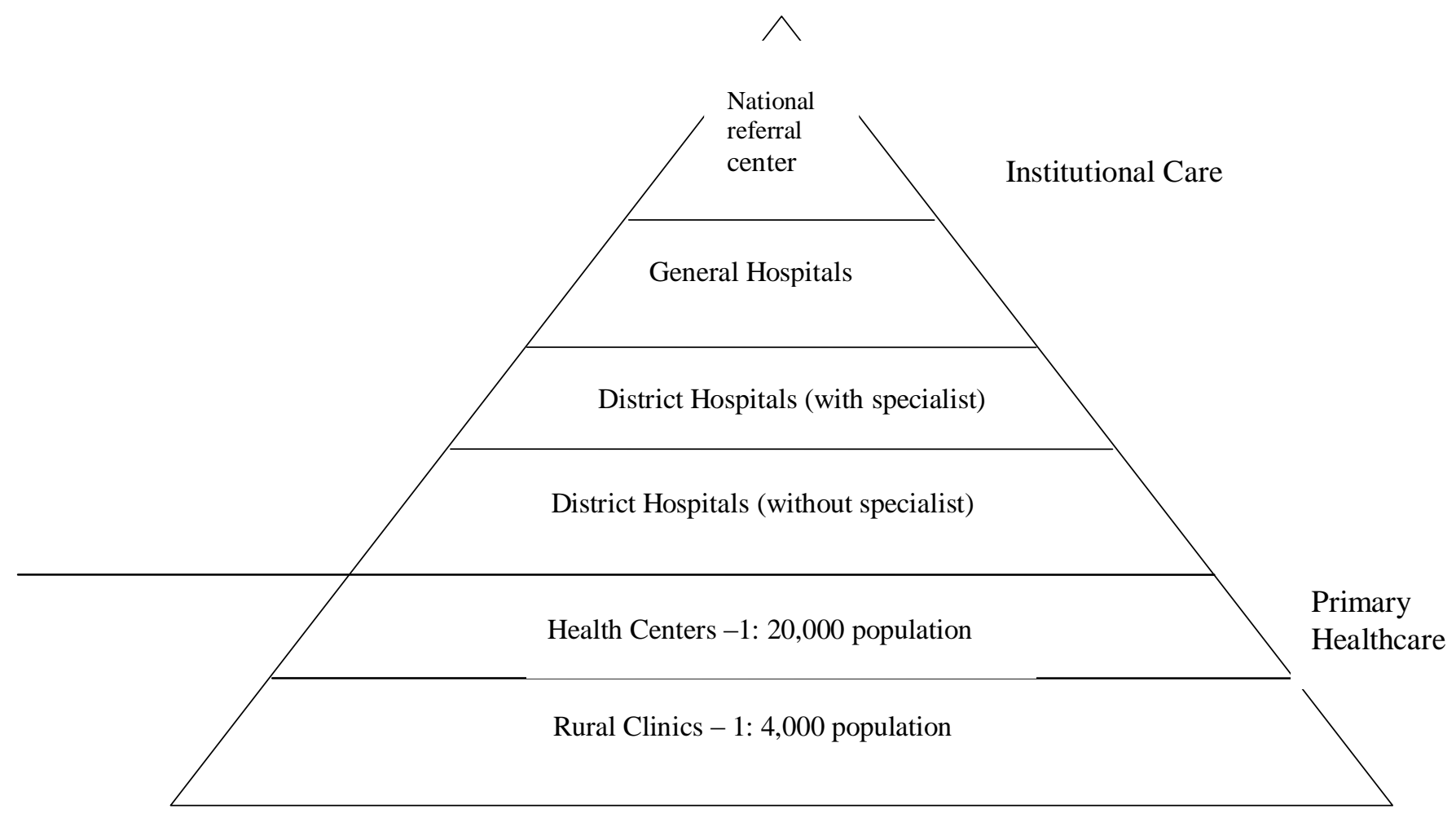

Figure 3. Healthcare delivery system in Malaysia MOH

Source: $\mathrm{MOH}$, reproduced with permission

The private health sector in Malaysia started four decades ago, comprising private hospitals, clinics, nursing homes, maternity homes, private medical practitioners, private dental practitioners and private pharmacists, private insurance companies and traditional 
medical practitioners (Nik Rosnah, 2005). The majority of medical practitioners (general practitioners) are in private practice and patients have to pay for the health service given by them (Poi et al., 2004). Traditional healers called bomoh or dukun for the Malay traditional practitioners and sinseh for Chinese are also part of the private system (Nik Rosnah, 2005). According to the World Health Organisation (WHO), the traditional healer $(\mathrm{TH})$

is a person who is recognised by the community in which he lives as competent to provide health care by using vegetables, animal and mineral substances and certain other methods on the social, cultural and religious background, as well as the knowledge, attributes and beliefs that are prevalent in the community, regarding physical, mental and social well-being and the causation of disease and disability. (WHO, 2001 cited in Razali, 2009, p. 14)

The access to healthcare services for people in rural areas is different from those people in the urban area. The public healthcare services are the primary contact for people from the rural areas. This is partly due to most of the private hospitals being located in urban areas. The private health services mainly serve the middle and higher income groups (Nik Rosnah, 2005). Many of whom work in the private sectors and the health services are funded by their employers. The utilisation of the health services shows that the lower income population is likely to use the public health service compared with the better off, reflecting wider differences within the last 20 years (Leng \& Barraclough, 2007). A recent Malaysian study revealed that factors that lead to underutilisation of healthcare services include being at a distance from health facilities, having limited family support during illnesses and being from the Chinese ethnic group (Krishnaswamy et al., 2009). Table 1 shows the various types of health facilities in the Malaysian healthcare system.

In the Malaysian context, for any kind of health problems, patients are allowed to go directly to any hospital or health clinic whether public or private; this is different from some other countries. To date, the policy of every patient needing to be registered with a primary care doctor or family doctor still does not exist. Ramli and Taher (2008) suggest that to facilitate effective coordination and continuity of care, legislation should be 
passed to require patients to be registered with one primary care doctor. This is particularly true for patients with chronic illness. Despite the increased prevalence of chronic diseases and the significant progress made in developing infrastructure, the primary healthcare service in Malaysia, public or private is still oriented to acute, episodic illnesses as well as maternal and child health (Ramli \& Taher, 2008).

Table 1: Health facilities in Malaysia Health Care System

\begin{tabular}{|c|c|c|}
\hline & No & Beds (officials) \\
\hline Hospitals $(\mathrm{MoH})$ & 130 & 33,004 \\
\hline Special Medical Institutions (MoH) & 6 & 5,000 \\
\hline Special Institutions $(\mathrm{MoH})^{1}$ & 2 & - \\
\hline National Institutes of Health $(\mathrm{MoH})$ & 6 & - \\
\hline Non-MoH Government Hospitals & 7 & 3,245 \\
\hline Private Hospitals & 209 & 11,689 \\
\hline Private Maternity Homes & 22 & 174 \\
\hline Private Nursing Homes & 12 & 274 \\
\hline Private Hospice & 3 & 28 \\
\hline MoH Dental Clinics & 1,707 & $2,910 *$ \\
\hline MoH Mobile Dental Clinics ${ }^{2}$ & 493 & $1,149 *$ \\
\hline MoH Health Clinics & 802 & - \\
\hline MoH Community Clinic & 1,927 & - \\
\hline MoH Maternal \& Child Health Clinics & 95 & - \\
\hline MoH Mobile Health Clinics & 193 & - \\
\hline Private Medical Clinics & 6,371 & - \\
\hline Private Dental Clinics & 1,435 & - \\
\hline
\end{tabular}

1 National Blood Centre \& Public Health Laboratory

2 Include Mobile \& Pre Dental School Team

* Dental Chairs

Source: MOH (2008) available at http://www.moh.gov.my/opencms/export/sites/default/moh. Reproduced with permission

The site of this research, HUSM is a teaching hospital and also functions as tertiary referral centre providing services including teaching and training for undergraduate and postgraduate students as well as health care. This hospital receives all the referred cases from each district hospital in Kelantan as well as throughout Malaysia and in particular 
from the three states at the east coast of Peninsular Malaysia with a 4.7 million population.

\section{Traditional health care system in Malaysia}

The WHO reported that approximately $80 \%$ of the world's population uses some form of T/CM. It is the basis of primary healthcare practice. In relation to the traditional practice, one of the objectives of the WHO is to encourage the integration of T/CM into the healthcare system. Historically, "traditional health care services have existed in Malaysia for centuries and comprise a variety of systems, namely Malays, Chinese, Indian, Thai and aboriginal systems" (Ariff \& Lieng, 2002, p. 101). The emphasis laid by the Malaysian government on the T/CM led to the establishment of the T/CM Division in the Malaysia MOH in 1999. There is increasing interest in integrating T/CM into modern medical practices. In relation to the traditional practice related to stroke care, the Malaysian $\mathrm{MOH}$ encourages research to be carried out on the use and effectiveness of complementary medicine or practice for post-stroke care to promote evidenced-based practice (NINH, 2006). Ariff and Beng (2006) report that traditional and medical practice are complementary to each other rather than antagonistic. The Malaysian healthcare system recommended that T/CM could be optimally integrated into the modern treatment. The recent development in T/CM show that the first public hospital in Malaysia, the Kepala Batas Hospital in Penang, started incorporating T/CM by offering services in acupuncture, post-natal massage (Malay traditional massage) and herbal oncology for cancer patients (The Star, 2007). The integration of T/CM into modern medical practice will be extended to other public hospitals in the future.

Traditional healthcare systems use bomoh or dukun for general illness and bidan kampong (traditional birth attendants) for providing postnatal care such as post-natal massage. Within the Malaysian context, bomoh could be categorised into four main groups based on their practices: herbalist, spiritual healer, bone setters and religious or

faith healer (Razali, 2009). Kelantan, where $90 \%$ of population are Malay, utilises a wide range of T/CM for treatment of various illness including musculoskeletal, postnatal massage and traditional massage for stroke. Many studies on the use of T/CM within the 
local population have been conducted. A study undertaken by Ismail et al. (2005) aimed to determine the health-seeking behaviour of elderly Malays living in rural areas of Kelantan, the utilisation of both modern and traditional medicines and the steroid content of the traditional medicine used. It is revealed that among 599 elderly respondents, $82 \%$ took medicines. The utilisation of modern and traditional medicine in the previous two weeks was $59.3 \%$ and $40.9 \%$ respectively. An almost similar percentage of utilisation of traditional medicine was found among pregnant women in the Tumpat district in Kelantan. Out of 210 mothers interviewed, 108 (51.4\%) used one type of herbal medicine during pregnancy.

With respect to traditional practice post-stroke, urutan tradisional is widely used among Malaysian people. However, there is no evidence or research to date to explore the reliance of people on urutan tradisional. Given the local culture and belief accepting various areas of T/CM, it is worth a study to be carried out to ensure safe practice. From personal experience, some people indicated that stroke patients do not need modern treatment without relevant evidence or studies to support this.

Traditional healthcare has been practised in many other countries worldwide (Razali, 2009). But to my knowledge, there is no mention about the use of traditional massage for post-stroke rehabilitation among the developed countries. A study undertaken in Thailand with almost similar socio-cultural practice with Malaysia revealed the use of massage by family caregivers for their stroke relatives as one of the prominent rehabilitation activities post-stroke (Jullamate et al., 2006). Furthermore, traditional medicine such as herbal medicine for stroke care has been practised in other Asian countries such as Korea (Kim \& Yoon, 1997). The findings of their research revealed that one third of residents of Seoul would choose herbal medicine if they suffered a stroke.

Based on my own working experience in the area of stroke rehabilitation in the Western countries, there is a marked difference in term of cultural and religious practice poststroke. Some of the key differences were related to the practice of traditional massage 
within the acute through the community setting as well as the involvement and contribution of families in caring for the family member with a stroke. This is partly influenced by cultural and religious practice among Malaysians.

\section{Healthcare services related to stroke patients in Malaysia}

Stroke patients, according to the Malaysian Clinical Practice Guidelines (CPGs) for Management of Stroke, are urged to be admitted via the emergency departments and be treated as having an emergency medical condition $(\mathrm{MOH}, 2006)$. For stroke patients who seek medical treatment at a national referral centre or teaching hospital with a neurological service, the pattern of healthcare service can be seen in Figure 4. The majority of stroke patients who are referred to this kind of hospital in Malaysia will be triaged to the critical or semi-critical zones (available at: http://www.nasam.org).

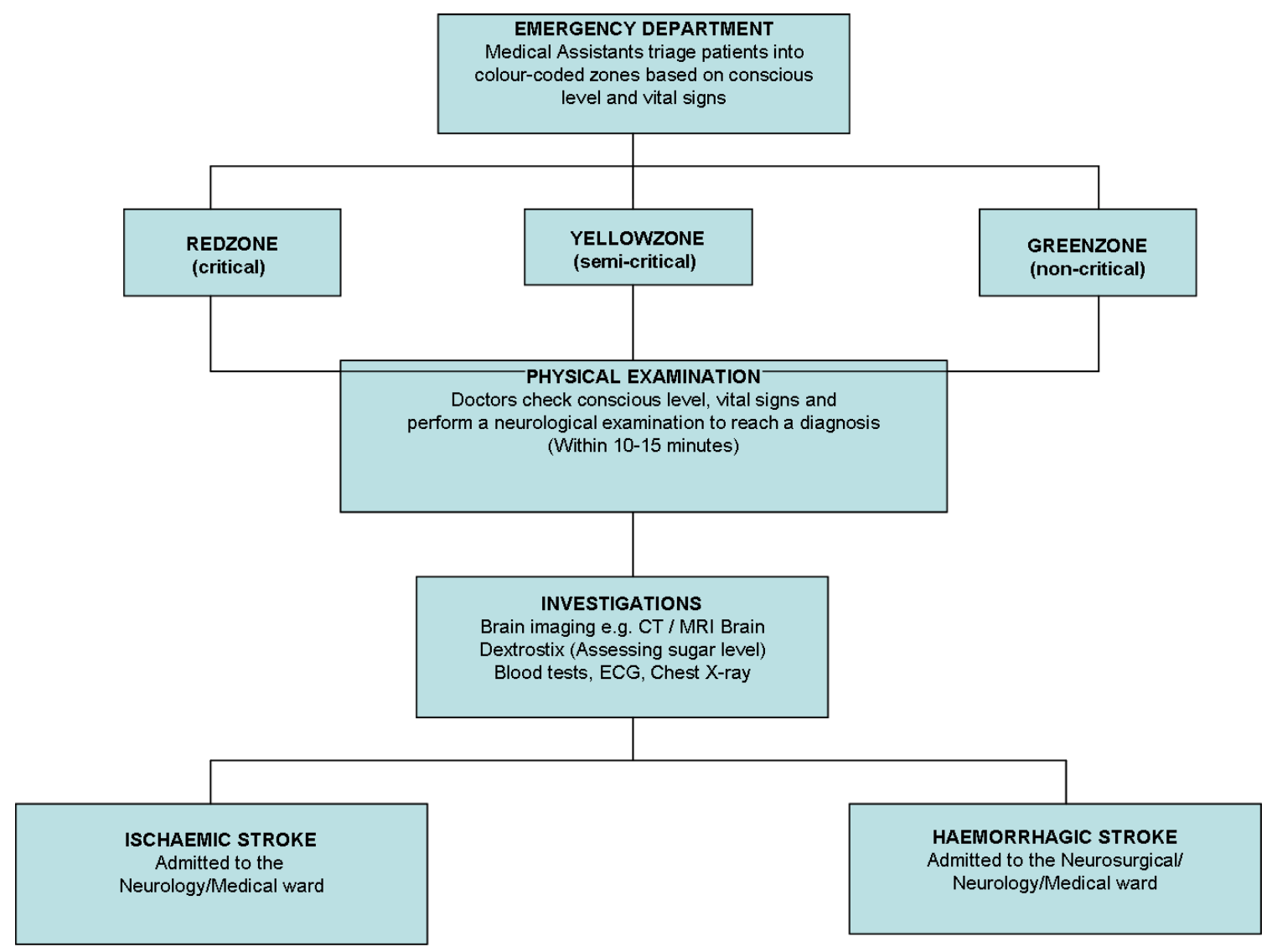

Figure 4. Flow chart for patient with suspected stroke

Source: http;//www.nasam.org, reproduced with permission 
Stroke patients who are admitted to a hospital without a neurological service are usually admitted in the general medical wards and are treated and cared for like other general medical patients (http://www.nasam.org, NIHM, 2006).

In relation to stroke care, evidence from the literature suggests that stroke patients are better managed in stroke units or centres which are run by multidisciplinary health professionals with specialised skills in stroke care (Cadilhac et al., 2004; McCann, Groot, Charnley, \& Gardner, 2009; Zhu et al., 2009). Specialised care reduces mortality and morbidity rates (Zhu et al., 2009). The literature highly recommended that healthcare providers and policy makers in each country should move towards the development of a stroke centre with a specialised multidisciplinary team, especially in less developed countries such as Malaysia. Apart from this, a comprehensive assessment of stroke patients with a multidisciplinary approach is necessary for an appropriate management of care to minimise the consequences of stroke.

Various strategies and actions have been implemented to prevent stroke and stroke recurrence and promote recovery and rehabilitation. These include promoting a healthy lifestyle and increasing public awareness related to the early recognition of signs and symptoms of stroke, improving stroke treatment and care, establishment of evidencebased practice and encouraging research on stroke. Apart from that the Clinical Practice Guidelines (CPGs) for Management of Stroke were developed to guide and assist in the management of stroke patients $(\mathrm{MOH}, 2006)$. These guidelines are regularly reviewed and updated using the latest evidence available.

The Malaysia MOH (2006) has indicated that patients are reliant on traditional therapies for post-stroke therapy. It is not known whether this might be one of the reasons for the public scepticism towards hospital management of stroke in Malaysia ( $\mathrm{MOH}, 2006)$. This raises a question on the need to explore various issues related to traditional healthcare practice as not much evidence is available locally. In addition, the Malaysian $\mathrm{MOH}$ encourages research to be undertaken clarifying patients' cultural beliefs among those who solely depended on traditional therapies (NINH, 2006). 
As there is no national stroke registry, specific local data regarding the incidence, prevalence and mortality of stroke are lacking (Hamidon \& Raymond, 2003; MOH, 2006). Furthermore, as stroke is an age-related disease, it is predicted that the incidence of stroke in Malaysia will increase in the future due to the increase in older people in the population (MOH, 2006). This is partly due to underlying factors such as hypertension, diabetes, smoking and stress (Hamidon \& Raymond). The increasing incidence of hypertension as a major risk factor for stroke also contributes to the increasing stroke incidence in Malaysia.

The advancement in medicine and public health, coupled with nutrition, has also contributed to an increase in life expectancy which then leads to demographic changes in the Malaysian population (Poi et al., 2004). For example, the number of Malaysians aged 65 years or older increased by an average of $2.4 \%$ from 2000 to 2003. It is well-known that this age group is more likely to suffer various kinds of complex medical problems or chronic diseases such as hypertension, diabetes, stroke or other chronic degenerative disease. On the whole, it can be seen that stroke is a major factor contributing to the mortality and morbidity rates in many developed and developing countries.

As stroke is one of the major burdens to the Malaysian healthcare system (Tan et al., 2006), the total cost of stroke management is high and is believed to be grossly underestimated. However, the accurate cost is unknown partly due to the absence of a centralised stroke registry in the country (NIHM, 2006). Therefore it is critical to find strategies to minimise stroke incidence, stroke recurrence and morbidity caused by stroke. The other important aspect is to enhance and facilitate optimum recovery and rehabilitation. Recently, the need for research on stroke patients was given attention by the Malaysian government, particularly the $\mathrm{MOH}$, to explore and understand what happens to stroke patients in the community (MOH, 2006).

Furthermore, studies show that effective stroke care by multidisciplinary teams using evidence-based practice can reduce stroke mortality and morbidity rates and stroke recurrence, as well as enhance recovery and rehabilitation (Venketsubramaniam, 1999; 
Williams, 2005). Therefore a group of health professionals from multidisciplinary teams in Malaysia have forwarded strong recommendations to prioritise stroke research in the country. In line with this, stroke research was considered as a research priority in the health sector in the Ninth Malaysia Plan (9MP) which provided the plans for 2006-2010 (NINH, 2006). The Malaysia Plan is laid out by the Malaysian government every five years. Prioritisation of stroke research is consistent with the two primary goals of 9MP which are to prevent and reduce disease burden and to enhance the healthcare delivery system ( $\mathrm{MOH}, 2005)$. A wealth of evidence from the studies in developed countries supports the importance of health information, education and support from various disciplines to enhance recovery and rehabilitation of stroke patients and their family members (Denby \& Harvey, 2003; Dobkin, 2005; Rodgers et al., 1999).

Furthermore, a group of multidisciplinary health professionals indicated that very limited resources to help stroke survivors and their caregivers are available in the community in Malaysia (NIHM, 2006). Therefore it is very important to explore the resources or support available for them in the community. It is common knowledge that a variety of support and resources are needed to help stroke patients and families for optimum recovery and rehabilitation.

In the current Malaysian healthcare system, having supportive family caregivers of stroke patients is very important as they are the core persons in providing care in the home setting. Therefore the focus of this study is on the patients and families in the real situation in the community.

The western practice of managing post-stroke patients in a stroke unit or stroke rehabilitation unit with expert nursing and allied health care personnel is very limited in Malaysia. Within the study setting, the rehabilitation unit provided rehabilitation service to all categories of patients who require rehabilitation. The types of services provided include physiotherapy, occupational therapy and speech therapy. According to Yim (2005) there is a misunderstanding of the role of the physiotherapist and occupational therapist due to some similarities between the two professions. This misunderstanding causes conflict among 
therapists and other health professionals themselves. The provision of rehabilitation services was based on the referral made by the medical officer, whether for an in-patient or outpatient who was referred for follow-up visit at the rehabilitation unit. Home visits have also been implemented but only a selected few patients get these due to budget constraint. Therefore it raised an issue on the utilisation of rehabilitation services by stroke patients who are discharged with various degrees of disabilities, particularly related to mobilisation, which might hinder them from access to these services. There is also an issue of whether comprehensive rehabilitation services were provided for stroke patients or whether some

patients missed out or were never referred for those services especially those from the rural areas.

\section{The family caregivers or informal healthcare providers of stroke patients in Malaysia}

Family caregivers for stroke patients include spouses, adult children, siblings, nieces or nephews and other extended family members who provide informal care for patients. Many published studies demonstrate that the majority of family caregivers of stroke patients are their spouse or partners and children (Anderson et al., 1995; Kalra et al., 2004; O’Connel et al., 2003; Pierce, Steiner et al., 2004; Rombough et al., 2007).

Similar to other countries, Malaysian stroke patients are discharged early, sometimes within one week. The responsibilities of on-going care are generally left to family members. It is well accepted among Malaysians that informal care is readily available by their spouse or family which can cause the patient to become dependent on their family (Poi et al., 2004). This is due to the fact that the majority of Malaysians aged 60 or older co-reside with their adult children although the household structures have been changing (Vanzo \& Chan, 1994). This is particularly obvious when the older parents are in a poor health condition and because of other contributing factors such as financial, cultural and religious practice (Vanzo \& Chan). As such, it is found that family plays a crucial role in the rehabilitation of stroke patients and as primary support persons. 
The above evidence is congruent with the findings from earlier studies (Martin, 1989). Family members become the primary support person or informal caregivers. Usually family caregivers will get assistance from other family members. As the majority of stroke patients are discharged home with some residual disabilities families have to play a major role in caring for stroke patients at home. The role of family caregivers includes providing basic care such as personal hygiene, toileting, assisting in mobility and rehabilitation as well as feeding and socialising. The suitability of the condition of the house to suit stroke patients is mostly the responsibility of the family members.

The family caregivers have to adapt and cope with the roles and responsibilities of caring for stroke family members in terms of the physical, psychological, emotional and financial aspects. In general, family members have to use their own coping mechanisms to deal with the burden caused by stroke. In the Malaysian context, to date, there is no available data showing how families of stroke patients cope and manage their stroke family members at home. About $90 \%$ of the Kelantan population are Muslim. There is no available data on cultural or religious practices influence on how patients and family caregivers cope with the consequences of illness. What are the types of resources needed to help them in their care giving roles? There is a gap in knowledge on how Malaysian stroke patients and their caregivers manage their everyday life, and how much support and resources are available and received to help them on a day to day basis.

I have observed that on occasion family caregivers have to stop paid work to look after their sick parents or family members. They have to use their own properties or savings to overcome financial problems. Some financial assistance is provided by the welfare service to families who fulfil certain criteria and are categorised as being in a poor or very low income group. In comparison with other western countries such as the United Kingdom (UK), which introduced the Carers Act in 1995 (Brereton, 1997), to date there are no clear guidelines or policies related to the caregivers' rights.

The demographic characteristics of the family caregivers such as age, ethnicity, gender, socioeconomic status or level of income, types of employment and their relationship with 
stroke patients may influence the types of coping mechanisms practised by family caregivers. Furthermore, the differences in the background characteristics may influence their caring experience. Of the 27.7 million people in Malaysia in 2008, 34\% are under the age of 15 years (http://www.moh.gov.my/opencms/export/sites/default/moh). It is also an interesting area to explore whether family caregivers include family members who are at a younger age. This in turn may reflect different problems compared with older family caregivers. In addition, it may reveal some other psychosocial consequences on the part of caregivers. A previous study in Toronto, Canada has demonstrated that the needs of younger caregivers are different from those of older stroke caregivers (Smith et al., 2008). While younger caregivers emphasised the need for training and information, older caregivers were focussed on the importance of maintaining a positive attitude and coping with the situation to the best of their abilities. Compared with other developed countries where many more stroke survivors are institutionalised or stay in their own home with their nuclear family, the role of Malaysian family caregivers greatly differ.

In relation to gender difference, Malaysian men and women caregivers are both involved in caring for their stroke family members. In a family which is comprised of men and women, the traditional role of women as primary caregivers in the family is still evident. For example, if the wife suffered from stroke, most of the time the adult daughter would co-reside with their older parents and become the primary caregiver.

Recent evidence has demonstrated different views. Nowadays, there is a growing awareness among Malaysians of the need for institutionalised care for their older relatives. It is partly due to insufficient family members to provide care at home as a consequence of a reduction in birth rates (Poi et al., 2004). In addition, many women now enter employment and live separately from their older parents (Poi et al., 2004).

Given the situation and the roles and functions of caregivers of stroke patients in Malaysia, it is worth examining the availability of community resources and rehabilitation support for stroke patients after hospitalisation. There is evidence that 
rehabilitation support is mostly provided by the private health service, which has fee-forservice basis (NIHM, 2006). It is common knowledge among Malaysians that the private health services cater for people from the middle and higher income groups. Unavailability of a stroke unit in the region and a lack of a rehabilitation centre in Malaysia may also impact on patients' recovery. In addition, some Malaysians believe and rely on the traditional methods of healing originating from cultural belief.

Generally, in Malaysia, family caregivers are assumed or taken for granted to provide care for stroke patients in their own home. This expectation is part of a cultural norm and practice as well as being part of the religious belief especially among the Muslims. In Muslim belief, adult children have the responsibility to look after their parents especially during illness. In most of the states in Malaysia especially within the study setting, the idea or practice of putting old or disabled parents in a nursing home is not well accepted.

In addition to caring for people who have suffered a stroke in the home, family caregivers in Malaysia are also involved in giving care to stroke patients in the hospital. They are not given formal training to do this. However, it is not known how much informal knowledge and skills are provided by the healthcare provider. While there has been considerable research on the provision of knowledge and skills for the caregivers from developed countries, little has been written on developing countries in particular Malaysia. Brereton (1997) and Brereton and Nolan (2000, 2002) suggest that healthcare providers need to play an active role in preparing the family caregivers for their care giving role. Therefore this research was necessary to provide an insight on the educational needs of patients and families post-stroke.

\section{Conclusion}

To summarise, the management of the stroke patients in hospital and after discharge needs to be examined from various perspectives such as their rehabilitation practice, the provision of information and education as well as support available. Rehabilitation is an integral part of stroke care. It is therefore necessary to examine how stroke patients 
manage and what kind of support is available for them in the community ( $\mathrm{MOH}, 2006)$. Apart from rehabilitation, there is evidence that stroke patients should be put under multidisciplinary care comprising nursing, physiotherapy, occupational therapy, speech therapy and dietician support. As stated in the working paper by a group of multidisciplinary professionals for the justification of the needs for stroke research in the 9th Malaysia Plan, there is a very limited community resource to help stroke survivors and their caregivers in the community (NIHM, 2006). Therefore there is a pressing need to know how stroke survivors and families cope with the consequences of stroke and resulting disabilities in Malaysia. To my knowledge, this is the first research project on the educational needs of Malaysian stroke patients and families at home-setting. It is very important as stroke research from the nursing perspective is still very limited. 


\section{Chapter 3: Literature review}

\section{Introduction}

In Malaysia, few studies have been done in relation to the health education needs of stroke patients and their families. The majority of research is on medical aspects of stroke such as stroke incidence, prevalence, mortality and morbidity rates as well as stroke risk factors (Jaya et al., 2002; Man et al., 2006; Ong \& Raymond, 2002). The previous chapter illustrated that families play a major role in post-stroke care at home. However, to date, there is no research on whether Malaysian stroke patients and family members are sufficiently educated and prepared on the life changes and homemanagement post-stroke.

This chapter begins with the literature search strategy that was used to find the relevant literature for this study. An overview of the concept of stroke and stroke statistics worldwide follows. The third section presents what is known about the health journey of stroke patients and their caregivers. This health journey of stroke patients and family caregivers commences with the experience and the impact of stroke on patients and their family caregivers. The aim is to provide an insight into the health needs of patients and their caregivers post-stroke. The appraisal of the literature provides important knowledge and insight and is used as a basis for the present study. Although much of the literature and context presented comes from western perspectives as studies on this area locally are lacking, it is important for identifying the gap in knowledge. Further sections focus on the studies of the educational needs of stroke patients and caregivers following stroke. Key limitations of the literature are discussed which could be beneficial for future research. This chapter concludes with an account of the areas needed for this study.

\section{Search strategy}

A few strategies were used to search for the relevant literature for this study. Electronic databases including the Cumulative Index of Nursing and Allied Health Literature (CINAHL), ProQuest, and OCLC FirstSearch (ArticleFirst, MEDLINE) were used. The 
key words used for the search included informal/family stroke caregivers, nursing education, stroke, CVA, stroke rehabilitation, stroke patients, stroke education, stroke knowledge and stroke recovery. Another important source of literature for me was to follow up references from reference lists from related journal articles and books. I found these latter sources very helpful as most of the articles were closely related. Relevant articles or books were also obtained from the Victoria University of Wellington (VUW) library catalogue, from my own collection, and given or suggested by supervisors, friends and other graduate students. I also searched unpublished doctoral and Masters theses in nursing education and related areas. One particularly useful source was an unpublished $\mathrm{PhD}$ thesis by Ali (2001) that provides useful insight into some of the issues surrounding traditional and complementary medicine (T/CM) in Malaysia. Other sources of information were obtained from newspapers and magazines especially related to the Malaysian context. I found that this information was useful, as it contained the latest information and developments in Malaysia. For example, information about the first government hospital in Malaysia that incorporates T/CM alongside modern treatment was published in the newspaper.

From this literature search, I found many articles on stroke patients and their family caregivers were written based on studies done in developed countries. Articles on the educational needs of stroke patients and their families span 15 years and are written by many authors (Brereton, 1997; Brereton \& Nolan, 2000; Denby \& Harvey, 2003; Dorsey \& Vaca, 1998; Eames et al., 2008; Eames et al., 2003; Garret \& Cowdell, 2005; Hanger et al., 1998; Hinojosa \& Rittman, 2009; O’Connell et al., 2003; O’Mahoney et al., 1997; Pierce, Finn et al., 2004; Wachters-Kaufmann et al., 2005; Wellwood et al., 1994; Wiles et al., 1998). In addition, extensive literature was found on the experiences of stroke patients and their families living with the impacts of stroke (Anderson et al., 1995; Bakas et al., 2004; Bakas et al., 2002; Bennet, 1996; Bluvol \& Gilboe, 2004; Bugge et al., 1999; Burton, 2000; Coombs, 2007; Eaves, 2000; Grant \& Davis, 1997; Hafteindottir \& Grypdonck, 1997; Han \& Haley, 1999; Hare et al., 2006; Hopmann \& Verner, 2003; Koch et al., 2005; Oloffson et al., 2005; Pierce et al., 2004; Pierce et al., 2006; Secrest, 2000; Ski \& O’Connell, 2007; Smith et al., 2008). 
No literature was found on the educational needs of stroke patients and their families in Malaysia. Information related to Malaysian traditional health care practice has isolated (Ariff \& Beng, 2006; Ismail et al., 2005; Rahman et al., 2008; Razali, 2009) and studies on the areas related to stroke and stroke risk factors such as hypertension or diabetes (Glamcevski, 2002; Glamcevski \& Tan 2001; Hamidon \& Raymond, 2003; Jaya et al., 2002; Ong \& Raymond, 2002; Man et al., 2006).

The literature search provided important insights on the health needs of patients and families particularly the need for education across the stroke trajectory. This search also provided an awareness of the limited literature on these areas within the study context as well as within other non-Western countries. The extensive review of the literature also assisted in designing the theoretical framework for this study.

\section{The nature of stroke and stroke statistics}

The term 'stroke' used in this thesis refers to any categories of stroke whether ischemic or hemorrhagic stroke. Stroke also known as "brain attack" is one of the most common medical emergencies (Adams, 1998; Brereton \& Nolan, 2002; Lott et al., 1999; MOH, 2006; Pierce, Finn et al.; 2004; Talbot et al., 2004; Wester, 1999; Williams, 2005).

Historically stroke was called "apoplexy" (Thibodeau \& Patton, 2002). Literature revealed that although stroke is a common medical emergency, it often is not diagnosed or treated as an emergency medical condition by general practitioners and is not always considered as such by the public (Smith et al., 2004). Indeed, myocardial infarction or trauma is more familiar as an emergency condition compared to stroke (Lott et al., 1999).

Stroke is an injury to the brain which has a great effect on an individual's health (Denby \& Harvey, 2003). Stroke occurs when certain areas of the brain are lacking blood supply or the blood supply is interrupted due to a blockage. Blockages cause ischemia or an infarct. Hemorrhagic stroke is caused when a blood vessel bursts (Lott et al., 1999; Mitchell \& Moore, 2004; Wong, 1999). Stroke is also defined "as a heterogeneous, neurological syndrome characterized by gradual or rapid, nonconvulsive onset of 
neurological deficit that fit a known vascular territory and that last for 24 hours or more" (Hickey, 2009, p. 589). Green and King (2009) conclude that "the simple definition of stroke is a disruption of blood flow to the brain" (p. 1199). For some, the concept of stroke can be accurately defined based on the detailed descriptions of the person who experiences it.

Furthermore, due to the specialised function of the brain and the distinct features of the impact of stroke, stroke can be differentiated based on the site of the brain damage. A left cerebrovascular accident (CVA) damages the left hemisphere of the brain and a right CVA damages the brain's right hemisphere. Physical deficits such as a weakness or paralysis will occur on the opposite side of the CVA site (Hayn \& Fisher, 1997). Individuals who have a left CVA may suddenly be unable to speak, have speech and language deficit or aphasia, and have an inability to follow directions, or move their right arm or leg (Dobkin, 2005; Hayn \& Fisher, 1997). Besides the physical deficit, patients may also experience functional deficits such as spatial and perceptual deficits. To summarise, the differences of the left and right CVA not only determine the physical impact but also many more functional differences among patients.

Ischemic stroke which is due to occlusion and thromboembolic events accounts for 70$80 \%$ of all strokes, and is less severe when compared to intracerebral haemorrhage which accounts for up to $20 \%$ of all strokes and results in death in more than $50 \%$ of the patients (Lott et al., 1999). Many studies reported that ischemic stroke is more prevalent than hemorrhagic stroke (Oloffson et al., 2005). A study by Thrift et al. (2001) on 245 stroke patients in Taiwan revealed that only $14.5 \%$ had a cerebral haemorrhage compared with $72.5 \%$ who had a cerebral infarction. Another study indicated that out of 86 stroke patients, 75 had a cerebral infarction and only 11 had intracerebral hemorrhage (Anderson et al., 2000). A similar pattern is found in William's (1997) study, whereby 96\% of 67 stroke patients had an ischemic stroke and only $4 \%$ had hemorrhagic stroke. The National Institute of Health Malaysia (NIHM, 2006, p.7) reported that "several studies on Asian patients showed that ischemic stroke represented $75 \%$ while hemorrhagic stroke represented $25 \%$ of patients with stroke". It supported the findings 
from a Malaysian study that was conducted at the HUSM to determine the types of stroke patients admitted to this hospital. It was revealed that out of 158 first-ever stroke patients who were admitted over one year, ischemic stroke and hemorrhagic stroke were $56.3 \%$ and $32.9 \%$ respectively (Jaya et al., 2002). This was similar to the types of stroke revealed in the study conducted at the Hospital University Kuala Lumpur (HUKL), an urban-based referral centre in Malaysia. On the whole, it can be concluded that the cerebral infarction is more prevalent among stroke patients than cerebral haemorrhage.

As with other chronic diseases such as cancer and heart disease, stroke shares the same kind of risk factors such as hypertension, sedentary lifestyle, obesity, smoking and unhealthy diet (Bonita, 2004). It is similar to the study done by Jaya et al. (2002) which showed almost the same risk factors such as hypertension, smoking, diabetes mellitus (DM), heart disease and previous transient ischemic attack (TIA). Besides that, another local study on 246 Malaysian stroke patients revealed that apart from hypertension and diabetes, hyperlipidemia is also a major risk factor for stroke (Ong \& Raymond, 2002). However, in Malaysia, it was found that there are differences in the prevalence of risk factors for strokes in the various ethnic backgrounds (MOH, 2006).

As an emergency medical condition, it is very important to be aware and understand the reasons why stroke patients need to be treated as an emergency to prevent extensive or further brain cell damage which can take from seconds to minutes or hours to occur. This is due to the differences in the speed of the brain cells to die which in turn depends on the distance from the stroke site. The brain cells further away from the site of the stroke or in an area called penumbra, may take a few hours for the cell function to decline, whereas those close to the site or at the centre of the infarct of stroke will often die quickly (Lees et al., 2000; Lott et al., 1999). Therefore the identification of the type of stroke should be investigated and treatment initiated immediately to minimise brain cell damage and to facilitate functional improvement.

Furthermore, Pollack and Disler (2002) have demonstrated that the functional improvement of stroke patients depends on a few factors or processes including neuroplasticity, resolution of cerebral oedema, recovery of part of ischemic penumbra 
and the patient learning to compensate by using the unaffected parts of the body. The speed and completeness of recovery following stroke varies and were partly dependent on factors including the severity and the type of stroke and the age and overall state of health of the individual (Lees et al., 2000; Thibodeau \& Patton, 2002).

Literature revealed that most of those who survive a stroke often need long-term followup and medication (Oloffson et al., 2005). Although it is commonly believed that as an age-related disease, stroke frequently occurs among the elderly, studies show that stroke also happens among younger patients.

Globally, it has been predicted that there will be an absolute increase of stroke incidence and burden caused by stroke in the future due to population aging (Butcher \& Frankish, 2006; WHO, 2003; Zwygart-Stauffacher et al., 2000). Statistics show that stroke is the leading cause of disabilities in the world (Henderson et al., 2007; WHO, 2003). It also causes an enormous burden to the healthcare system worldwide. As there is an advancement of stroke treatment and care many more patients survive which then leads to an increasing number of patients discharged with some residual disabilities. Therefore stroke is a major factor in contributing to morbidity rate in many countries.

Studies in western countries show that stroke is the most important cause of morbidity and long-term disability in Europe and the third most common cause of death in many industrialised countries. In the United States of America (USA), stroke is the leading cause of adult disability with up to $50 \%$ of stroke survivors incurring partial or total dependence in daily living (Smith et al., 2004). In Germany, the overall incidence of stroke is estimated to be 127,000 a year, $75 \%$ of whom are people having first strokes (Lott et al., 1999). Furthermore, stroke is the leading cause of death and disability in British Columbia (Cloutier-Fisher, 2005).

In two of Malaysia's neighbouring countries - Australia and Singapore - stroke is the third highest cause of death and also the leading cause of chronic disability in adults (Pollack \& Disler, 2002). In Singapore since 1970, stroke has accounted for $10 \%$ to $12 \%$ 
of all deaths annually (crude death rate of 50 to $60 / 100,000$ ) and has contributed to morbidity statistics (Lateef \& Anantharaman, 2005; Venketsubramaniam, 2005). This is similar to the findings from Jaya et al. (2002) among the Asian population with first-ever stroke at HUSM in Malaysia. Out of 158 first - ever stroke patients studied, approximately one-third died during the first month. The overall mortality rate was $37.3 \%(\mathrm{n}=59)$. This means that the survival rate is much higher than the mortality rate. Those who survive from stroke have to face a very challenging life living with residual disabilities caused by stroke.

\section{Stroke patients' health journey: The experience of the impacts of stroke}

Due to advancements in stroke care, the mortality and morbidity rates caused by stroke are declining but the effects of stroke remains devastating (Dorcey \& Vaca, 1998). They may last for several years. Due to the unique function of the brain, any pathological or physiological changes in any part of the brain usually result in various physical, psychological and emotional disorders in stroke victims. Stroke that occurs or damages either side of the brain does not only cause a functional deficit of the opposite site of the body, but also the entire brain suffers from the loss of communication with the injured portion (Dorcey \& Vaca).

Any experience will be best expressed or described by the person or individual who has experienced it. In relation to stroke patients, some of them may have difficulty in communication or aphasia especially during the acute stage. They may also be confused, experience short term delirium or dementia as well as have a cognitive deficit as consequences of stroke. As stroke is an age-related disease, the majority of those affected are among the older population and may develop dementia. These limitations become a barrier in getting the real experience or accurate information from patients. In this situation, they have to be excluded from studies due to their inability to communicate. In many cases, the experience of stroke survivors can be obtained from their family caregivers or primary support person. 
The experience of loss in many aspects of the patients' lives following stroke was revealed in a phenomenological study based on interviews with 15 stroke patients and their health professionals' documentation covering the first year of their stroke. From the integrated analysis of these two groups of participants, stroke patients were found to have lost their normal function as a consequence of stroke (Bendz, 2003). However, the meanings of loss were found to be more comprehensive among stroke patients compared with the health professionals' views. Patients' described losing control of their lives in terms of being unable to control their body and muscles, and feeling numb and strange. Besides this, a decreased capacity to recall, to concentrate and to retain knowledge had also affected their lives and sometimes led to a feeling of uncertainty. Furthermore, the loss of energy or fatigue had negatively influenced their daily lives as they were easily exhausted without specific reason. In turn, the health professionals' transcripts revealed their main interest was the experience of patients' reduced physical function following stroke. This study provides an insight into the need to consider and better understand the impact of stroke from the patients' perspectives. Health professionals and patients have to reach agreement on appropriate goals and activities and work towards those goals for optimal rehabilitation. Other studies also revealed that stroke patients often experienced living with loss and a feeling of insecurity in the aftermath of stroke (Burton, 2000; Downswell, 2000; Hafteinsdottir \& Grypdonck, 1997). Other findings from a qualitative study show that some stroke patients were hardly aware of or did not recognise the symptoms of stroke (Olofsson et al., 2005). The common symptoms of stroke include the sudden onset of weakness or paralysis of one side of the body (hemiparesis/hemiplegia), slurred speech, difficulty swallowing and headache.

Patients' experiences of living with stroke through all stages of the stroke process were described following interviews with eight patients and 18 caregivers (Eaves, 2000). The stroke survivors ranged in age from 56 to 79 years and had been discharged from the stroke rehabilitation centre of a community hospital in North Carolina (within the previous four months, following the stroke event). All the study participants were interviewed in their own homes using a semi-structured interview guide and focused observation. Data analysis based on the qualitative text analysis software package 
revealed five themes: discovering stroke, delaying treatment, living with uncertainty, discovering the impact of stroke, and reconstructing life. The theme 'discovering stroke' explained how stroke survivors experienced and started to recognise stroke symptoms such as weakness of the leg and arms, voice changes, light headedness and swallowing difficulty. The delay in getting treatment comprised six patterns: waiting, keeping secrets, convincing, verifying, and seeking care and consequences of waiting. Waiting is when a few patients endured their stroke symptoms for several days before seeking treatment. The act of keeping secrets revealed how patients attempted to prevent family members knowing their stroke symptoms. Convincing described how family members attempted to persuade the patient to seek treatment. The patterns of verifying and seeking care revealed how families attempted to verify the stroke symptoms including checking the blood pressure before seeking treatment. The consequences of waiting demonstrated that delay treatment for a few days may lead to severe stroke or earlier treatment may prevent stroke. The delay in getting treatment supports findings from previous research (William, 1997). Moreover, the theme of living with uncertainty may be related to the experience of loss and unpredictable prognosis or outcome of stroke. Finally, the impacts of stroke were explained as a loss of functioning and autonomy such as the inability to perform self-care tasks and struggling to be independent. The theme of reconstructing life was used to demonstrate that the stroke patients gradually developed life-long adjustment and adaptation to living with residual disability caused by stroke.

The study by Olofsson et al. (2005) aimed to examine stroke patients' experiences of the whole process from their falling ill to their returning home after the hospital care. This study involved in-depth interviewing with nine stroke patients who had a stroke within the previous four months and had been discharged from the hospital. The findings revealed that stroke patients' experience can be described under three main categories: 'responsible and implicated', 'depersonalised object for caring measures' and 'the striving for repersonalisation and autonomy'. Once stroke happens, most of the patients took responsibility to consult people close to them and seek care. Being in hospital was perceived as entering into the medical world. The patient is placed in a passive, dependent position and becomes an object for the caring measures. Patients felt a loss of 
autonomy and were not given much freedom in decision making. Being able to return home is rewarding for them in their effort for repersonalisation. To them, 'coming home' was an important factor for their recovery. Findings also revealed the patients' need for information on their illness, recovery, prognosis and medication. Once at home, the majority of them wanted advice, support and information on their state of health.

Ellis-Hill et al. (2009) studied 20 patients' and 13 caregivers' experiences of being discharged from hospital following a stroke. Key findings were that discharge was a significant moment in peoples' recovery. However, the quality of the experience was influenced by the level of support and information people had. Those who were considered they had sufficient knowledge and support had a more positive experience. A group of participants considered they were "in the dark about how to manage their physical recovery" (p. 68).

Furthermore, many studies have shown that stroke also has a great impact on family members of stroke victims (Bhogal et al., 2003b; Cameron \& Gignac, 2008; Dorcey \& Vaca, 1998; Dowswell et al., 2000; Exall, 1999; Hayn \& Fisher, 1997; Mitchell \& Moore, 2004; Perry \& McLaren, 2003; Sundin et al., 2000). The suddenness of the effects of stroke leads to various negative consequences to stroke victims and close family members. Family members were found unprepared for or lacked time to prepare for the care-giving role (Kalra et al., 2004; Walling, 2005). This unpreparedness can create a family crisis and impact on how a family functions, and can affect stroke rehabilitation (Bhogal et al., 2003b). The stress and burden faced by family members may influence the recovery rate of stroke patients. In addition, stroke can leave stroke survivors with severe residual disabilities (Clarke, 2002), which create a burden on families. It can lead to a great deal of problems in their daily life coupled with low financial status for the majority of them. These findings raise an important question that is addressed in this current research regarding the extent to which Malaysian stroke patients and their caregivers face similar problems. 
As stated by Bonita (2004), two-thirds of people who have stroke live in low and middle income countries. The financial burden caused by stroke coupled with the existing low income can create problems for stroke patients. Therefore many studies have been done to gain understanding of these problems and patients' needs while in the process of rehabilitation and recovery. Due to considerable economic hardship post-stroke faced by families in China, health insurance is needed to protect families against the higher healthcare cost (Heeley et al., 2009). Furthermore, findings from systematic reviews on 78 studies revealed that $24 \%$ to $33 \%$ of social consequences of stroke for working-aged people are economic difficulties (Daniel, Wolfe, Busch \& McKevitt, 2009). The impact of stroke on financial status may support some stroke patients' view that returning to work as an important indicator of recovery. For those who had not returned to work felt that paid employment was desirable but they were unable to overcome the barriers such as experienced fatigue and speech difficulties (Alaszewski, Alaszewski, Potter \& Penhale, 2007).

There are many variables that determine the impact of stroke on individuals such as age, coping patterns, severity of stroke, financial status and psychosocial consequences (Koch et al., 2005). Mitchell and Moore (2004) pointed out that the severity of the effects or the clinical symptoms of stroke depends on the location and function of the affected area of the brain and the extent of the damage. The physical, psychological, social and emotional impacts caused by stroke often disturb the daily activities of stroke survivors from mild to very severe impacts. The majority of stroke survivors need assistance in the activities of daily living (ADL) such as mobility, feeding, toileting and personal hygiene.

The effects of stroke on stroke survivors can be categorised into four main areas: physical, psychological, psychosocial and cognitive effects. However, the various effects of stroke are not isolated; they are influenced by and interrelate with each other. 


\section{The physical effects}

The physical effects of stroke are more obvious compared with other effects such as psychological, psychosocial and cognitive. These will be discussed later in the chapter. Physical deficits or limitations experienced by stroke patients include immobility or impaired mobility, weakness or paralysis of one side of the body, loss of energy, fatigue, dysphagia, incontinence, pain, speech and language deficits or aphasia and impaired arm function (Hayn \& Fisher, 1997; Koch et al., 2005). Dysphagia affects about 50\% of stroke patients (Royal College of Physician [RCP], 2004).

Generally, the more obvious impairment caused by stroke is related to mobility. One of the common changes following stroke is the balance disability (Tyson et al., 2006). Tyson and colleagues conducted a detailed descriptive study to examine the balance disability of 75 hospitalised stroke patients. Their findings revealed that more than $80 \%$ of the first-ever stroke patients had balance disability during the acute period. The most important impact on balance was sensation and weakness. However, other factors also may affect balance such as motivation and other co-morbidities.

The other common physical effect of stroke is impaired arm function (Lincoln et al., 1999). This usually occurs on the opposite side to the site of the stroke (Goulding et al., 2004). Stroke patients may experience pain of the affected arm due to improper handling known as shoulder pain syndrome. This is due to weakness or paralysis as well as pain which may lead to contractures (Hayn \& Fisher, 1997). The other type of pain is central post-stroke pain (CPSP) which occurs on the same side of the CVA (Hayn \& Fisher). Shoulder-hand syndrome or central post stroke pain most commonly occurs one or two months after stroke, whereas pain from contracture such as finger and knee flexion contracture can occur at any time (Hayn \& Fisher). Pain from shoulder hand syndrome will reduce stroke patients' quality of life (Lincoln et al.).

Disturbance or changes in many aspects of sexuality following stroke, including sexual functioning, libido, sexual arousal, sexual activity, coital frequency and satisfaction with sexual life, have been reported in published studies (Choi-Kwon \& Kim, 2002; Kimura 
et al., 2001; Korpelainen et al., 1998; Korpelainen et al., 1999). Choi-Kwon and Kim reported that out of 70 non-depressed and sexually active stroke patients, there was a considerable decline in sexual activities including libido, erectile function and coital frequency in $49 \%, 65 \%, 26 \%$ of the patients respectively, three months after stroke. Apart from that, the psychosocial changes following stroke may influence the sexual functioning of stroke patients as highlighted by Korpelainen et al. (1999). Their study aimed to assess the effects of stroke on sexual functioning involving 192 stroke patients and 94 spouses. The results demonstrated a significant decline in satisfaction with sexual life, libido, sexual arousal and coital frequency in both stroke patients and their spouse. A few psychosocial factors were found associated with sexual dysfunction such as fear of impotence, the patient's attitudes toward sexuality, the level of functional disability following stroke and the ability to discuss sexuality.

Speech and language deficit or aphasia were divided into three main categories: nonfluent aphasia (Broca's aphasia), fluent aphasia (Wernicke's aphasia), and global aphasia (Hickey, 2001; Liechty \& Garber, 2004). The different types of aphasia depend on the stroke site or the area of the brain damage which also causes different impairments. For example, patients with Broca's aphasia who suffered damage to the frontal lobe or specifically at the language centre within the dominant side of the brain had expressive aphasia. Some of the characteristics of expressive aphasia include patients speaking in short, meaningful phrases taking great effort to produce, experiencing difficulty to convey thoughts through words or writing and difficulty putting words together in coherent, grammatically correct sentences. However, they can understand other people's speech quite well. In contrast, Wernicke's aphasia, which results in receptive aphasia, is due to damage to a language centre located in a rear portion of the brain. The major problems caused by this type of aphasia are related to understanding spoken or written language and patients usually have incoherent speech. Dobkin (2005) indicated that 20\% of stroke patients experienced problems in communication in terms of difficulty to understand or to express language and should receive speech therapy. These physical disabilities can lead to emotional and psychological disturbances such as anger, anxiety, frustration, hopelessness and then, to depression. 


\section{The psychological effects}

The sudden onset of disability or loss of body functions experienced by stroke survivors can cause psychological disturbances to most of them. The psychological effects of stroke are more hidden compared with physical effects (Dorcey \& Vaca, 1998). Furthermore, unlike physical effects, the psychological effects on the stroke victims occur more gradually (Dorcey \& Vaca). Some of the examples of psychological effects of stroke are shock, fear, insomnia, frustration, loneliness, sleeplessness, nightmares and depression. Most of the psychological effects experienced by stroke patients lead to depression.

Errikson et al. (2004) conducted a national survey of 15,747 stroke patients in Sweden to explore self-reported depression after stroke, risk factors and the use of antidepressants. From this large population study, it was revealed that one in seven patients experience depression. In addition, it was reported that half of the depressed stroke patients used an antidepressant drug. The strength of the study is that it is a very large population study. On the other hand, it has a weakness in that the data were based on self-reported depression, not on a confirmed diagnosis.

Other studies describing stroke victims' psychological effects have been carried out. For example, Roomruangwong and Thavichachart (2005) found that anxiety and depression were common among stroke patients. There are also many factors that contribute to the experience of anxiety. Roomruangwong and Thavichachart conducted a study to find the association between anxiety and activities of daily livings outcome of stroke patients. This study was conducted with 85 stroke patients from a rehabilitation unit at Chulalongkorn Memorial Hospital, in Thailand, who attended the rehabilitation programme. The findings indicated that there was a strong association between lower level ADLs, longer duration of stroke and poor supporting system with anxiety. This finding was consistent with those from other studies (Chemerinski, 2001; Fuh, 1997). It was suggested by Roomruangwong and Thavichachart that although anxiety is one of the common reactions or responses of illness among stroke patients, it should be detected 
and managed early because undetected and untreated anxiety may influence and disturb the rehabilitation and recovery process. In addition, prolonged anxiety may lead to more serious conditions such as depression (Glamcevski \& Tan, 2001).

Moreover, there is increasing awareness and concern among nurses and healthcare providers of psychological effects of stroke such as anxiety, depression and shock besides physical effects. A study conducted by Bennet (1996) examined how nurses in a stroke rehabilitation unit attempted to meet psychological needs of depressed stroke patients. It was revealed that nurses were aware of the psychological needs of stroke patients and able to identify those who had depression. They were trying to fulfil their needs but they had some limitations due to lack of time and appropriate training. It was suggested that healthcare providers should be equipped with specialised skills and training in stroke care. It seems that the amount of nursing time spent and level of skills needed had to be taken into consideration in order to meet patients' psychological needs.

Some other studies were conducted with to determine the prevalence of depression and to understand the experience of depression among stroke patients or the factors associated with or leading to depression. One example is a local study conducted by Glamcevski (2000) on 80 stroke patients at 3-6 months post-stroke at University Malaya Medical Centre, Malaysia. It was found that of the 80 stroke patients, $53(66 \%)$ of these patients suffered depression. Of these 53 with depression, 41 (51\%) had mild depression and $12(15 \%)$ suffered a moderate to severe level of depression. This study also revealed a few factors that contributed to depression such as poor performance in ADLs, advancing age and the inability to presume pre-stroke life styles. This shows that depression is common among Malaysian 3-6 months after stroke. In addition, none of the patients were diagnosed and treated by the attending physician for depression before the study. This is likely in part due to lack of awareness. This supports the finding of an earlier study undertaken by Astrom et al. (1993) in relation to the factors associated with depression. The most important predictors of immediate major depression were left anterior brain lesion, dysphasia and living alone. Whilst dependence in ADLs was the 
most predictor of depression at 3 months post-stroke, it was not associated with immediate depression.

Fuh (1997) also revealed that depression was common after stroke, and that reduced performance in activities of ADLs and living alone were important contributing factors. Being dependent on others for ADLs caused patients low self-esteem and frustration which led to depression. Psychological effects on stroke patients can reduce motivation and self-esteem leading to a slow rehabilitation and recovery process. Besides those factors, Hafteinsdottir and Grypdonck (1997) indicated that the inability and difficulty in communication (dysphasia) can also contribute to depression as well as other consequences.

The above finding supports the finding from a study done by Addington-Hall (1995) with 237 informal caregivers. Addington-Hall found that most stroke victims do not receive appropriate care or treatment for their psychological problems. Some of the patients showed lack of motivation in rehabilitation activity, caused by depression. A study by Chemerinski (2001) found a similar result. This study was done with 55 stroke patients with minor and major depression to find the association between ADLs and depression. It was revealed that the remission of post-stroke depression was associated with improved ADLs. It was suggested that depression should be diagnosed and treated at a very early stage.

Surprisingly, although many studies indicated that depression needed to be treated, some researchers had another view. They considered that a period of depression is normal after stroke but intervention needs to be implemented if it disturbs the rehabilitation process (Hayn \& Fisher, 1997).

\section{The psychosocial effects of stroke}

Some studies show that there are various factors that contribute to social problems among stroke patients. Some of these are loss of employment, aphasia, incontinence, dysphagia, immobility, low self-esteem, loss of control, fatigue and fear of relapse 
(Alaszewski et al., 2007; Bendz, 2003, Corr \& Wilmer, 2003; Perry \& McLaren, 2003). These become a barrier for stroke patients to socialise and reintegrate into the community activities. In comparison with physical effects, psychosocial effects are more significant among stroke patients (Burton, 2000).

There are many factors that contribute to the level of psychosocial impact of stroke such as the patient's perception of the stroke, availability of financial resources, support and response from close family members, previous experience and coping mechanisms (Bhogal et al., 2003a). Some of the common psychosocial impacts are depression, loss and grief, social isolation, communication difficulties, shock, altered stability and irritability and a sense of rolelessness (Burton, 2000; Downswell, 2000; Hafsteindottir \& Grypdonck, 1997). Loss of function and a sense of rolelessness are due to the inability to return to the active life lived before the stroke (Downswell, 2000). Furthermore, the social consequences following stroke included negative impact on family relationship, deterioration in sexual life and leisure activities as well as financial hardship (Daniel et al., 2009).

In addition, the physical effects of stroke also contribute and lead to psychosocial problems. For example, difficulty in swallowing (dysphagia) can cause embarrassment (Anderson, 1992; Pound, 1999). This situation might disturb and result in limitations in social activities and the ability to become involved in community activities due to embarrassment and reduced self-esteem. Furthermore, the inability to do pre-stroke activities or having to give up work due to hemiparesis or hemiplegia can cause financial problems or social isolation which can lead to stress or depression.

Generally, in comparison with other chronic diseases such as cancer or heart disease, stroke frequently affects people's ability to socialise. The obvious reason might be language and cognitive deficit and physical disabilities which will have a great impact on the ability to communicate and mobilise which in turn leads to social isolation. As stroke causes sudden life changes for the patient and close relatives, emotional support is 
essential in every aspect of care to enhance the recovery process and improve their quality of life.

\section{The cognitive effects}

A few studies revealed that $60-80 \%$ of stroke patients were left with cognitive problems (Tatemichi, 1994). These will affect the stroke patient's life and cause the deficits in using language and thinking. Most stroke patients were having difficulty in using spoken language or difficulty in communication. In relation to the language ability and processing, stroke patients frequently have difficulty expressing needs and feelings, understanding information and conveying emotions. This situation may lead to patients being in a stressed condition.

Apart from the above issues, stroke patients also experience other cognitive deficits such as mental slowness which causes disabilities in their daily lives and further physical and emotional problems (Winkens, 2006). For example, the cognitive deficit faced by younger stroke patients may more severely affect their lives compared to older patients. Cognitive disability may influence the intellectual ability, memory and impede social activities.

In summary, the various effects of stroke were highlighted and these are interrelated and influence one another. It shows that stroke patients and their families need holistic care, support, information and education on how to cope with the effects of stroke. Given the multiple effects of stroke on patients, this study sought to examine the educational needs of patients and families post-stroke in order to maximise recovery and minimise complications.

\section{The impacts of caring for a loved one who has experienced a stroke}

Many studies demonstrated that stroke can cause devastating effects not only for stroke patients but also for their carer. Therefore stroke can also be considered as a family illness (Bakas et al., 2004; Brereton, 1997; Brereton \& Nolan, 2002; Exall, 1999; Mackenzie et al., 1998; Pierce, 2000; Smith et al., 2004; Sundin et al., 2000; Zwygart- 
Stauffacher et al., 2000). The burden of caring for their stroke family members may affect their health status. Some studies show that family members of stroke patients usually take on their role suddenly and without enough preparation (Brereton \& Nolan, 2000; Kalra et al., 2004; Smith et al., 2004; Walling, 2005). Some of the family caregivers experienced poor mental health when stroke patients were discharged home early (Anderson et al., 2000). Therefore it is very important for the healthcare providers to take an initiative to assess whether caregivers have been prepared adequately for the caring roles when discharged. The problems faced by the family caregivers will have a great influence on stroke patients because they are the main healthcare provider. They play a major role to facilitate recovery and rehabilitation.

Most of the studies done in western countries stated that caring for stroke survivors had a great impact on the caregivers in the aspects of physical, social and emotional and altered health status. Most caregivers also found that they lacked the knowledge and skills to care for the stroke survivors at home (Smith et al., 2004).

As mentioned, stroke affects the physical, emotional, psychological and psychosocial well-being as well as financial constraint of stroke patients and family members. In addition, due to the inability or limitations in performing ADLs and some other disabilities caused by stroke, the majority of stroke patients rely heavily on their family members especially their spouse or next of kin for the continuity of their life (Anderson et al., 1995; Pierce et al., 2006). Furthermore, the burden of caring for stroke patients is increased when stroke patients are discharged and sent home with some residual disabilities. Once home, this is the time where family caregivers start to realise of the full impact of caring for stroke patients.

Due to advances in stroke care, there is a reduced length of hospital stay for stroke patients and the number of stroke patients who survive with disabilities has increased (Walling, 2005; Zwygart-Stauffacher et al., 2000). Therefore much of the on-going care for stroke patients takes place in the community or in their own home (Foster \& Young, 1996; McBride et al., 2004; Pierce, Steiner et al., 2004). This care may take a few 
months or years (Bluvol \& Gilboe, 2004). With long-term commitment in the caregiving roles, caregivers have to face the reality and challenging role of caring for stroke patients in the community with limited support and resources (Dorsey \& Vaca, 1998). Therefore it seems that both healthcare providers and family caregivers have to play a significant role in order to facilitate maximum recovery. They also have to support patients with residual disabilities reintegrate and readapt into the community. Mayo et al. (2000) argued that one of the aims of stroke management is to facilitate community reintegration.

Although the majority of the studies related to stroke focused on stroke patients, not their spouse or caregivers (Bluvol \& Gilboe, 2004), there are some studies on the experiences of caregivers in caring for stroke patients which are significant to highlight in relation to this study. Most of these studies focused on the burden or problems and needs of family caregivers in the community using a qualitative approach. This approach is more appropriate for this kind of study because it concerns the subjective experiences of study participants. The qualitative method of data collection has more advantages to obtain data related to people's experiences than the quantitative method (Streubert \& Carpenter, 1999). Semi-structured and in-depth interviewing are popular tools to collect data, and what I applied in my study.

Mackenzie et al. (1998) studied how 13 nurses in Hong Kong assessed the problems and needs of caregivers in caring for stroke patients at home. The study involved the observations of the caregivers' verbal interaction and facial expression, caring abilities, relationship with family, willingness to care, own health and knowledge of the stroke patients condition as well as their house condition. These nurses indicated that the strategies used were communication with patients and caregivers and by observation.

A wealth of evidence suggests that caring for stroke patients is burdensome and affects the health of informal carers (Anderson et al., 1995; Mackenzie et al., 1998). The impacts on the caregivers that are mostly reported are the negative impacts related to social, emotional, reduced quality of life and altered health status. In terms of social 
impact, caregivers may have to give up a job to look after stroke patients which can lead to financial problems. Caregivers may also experience isolation from the community activities due to lack of leisure time. Anxiety, stress and depression are examples of the emotional impact faced by them. Bakas et al. (2004) revealed that caregivers are at risk for negative health outcomes such as depression, psychosocial impairments, and even mortality as a result of providing care for their family members with a stroke.

An enormous burden caused by stroke may place their caregivers at substantial risk of altered health status. This has been demonstrated in many published studies. Han and Haley (1999) reviewed several research articles related to family care-giving for patients with stroke. One of the goals of the review was to explore the effects of care giving on caregivers' well being. Seventeen of 20 studies measured psychological distress particularly depression among caregivers. Different types of instruments were used to measure depression among caregivers such as Center for Epidemiologic Studies Depression Scale (CED-D) (Epstein-Lubow, Beevers, Bishop \& Miller, 2009), General Health Questionnaires (Lincoln, Nicholl, Flannaghan, Leonard, \& Van der Gucht, 2003), and Hamilton Depression Rating Scale (Mitchell et al., 2008). Despite various instruments used the studies clearly indicated that caregivers experienced high level of stress in their role, which in turn leads to depression. Due to small sample sizes, the findings cannot be generalised. However, it has been suggested that depression among caregivers of stroke patients can be reduced by various interventions. Such intervention includes frequent home visits by stroke nurse specialists (Foster \& Young, 1996).

Secrest (2000) studied the experience of primary support persons (PSP) or primary caregivers in caring for stroke patients. This study explored the nature and the quality of experiences which focused on the meaning of living with stroke patients. Data were collected from 10 PSP of stroke patients who had completed inpatient rehabilitation and were living at home and at least six months after stroke. This study had employed the phenomenological approach using in-depth interviews. Secrest's findings show that the meaning of the experiences concern fragility, vigilance and loss/responsibility. To them, life was perceived as fragile. They had to be vigilant about bad consequences of stroke 
such as death, recurrence of stroke or any possible poor prognosis of stroke. Apart from that all the participants indicated that personal fragility is related to their aging and illness. Most of their experiences are related to a feeling of loss.

Due to the long-term commitment in the care-giving role, some researchers have explored whether the problems faced by caregivers change over time. One study was conducted by Pierce et al. (2006) on nine adult caregivers for stroke patients living in rural Ohio and Southern Michigan. These researchers found that caregivers faced a lot of problems related to their care-giving roles. The unexpected changes on the life of family caregivers created considerable stress. Moreover, it also produced some coping mechanisms among them. Although a small study, the findings provide us with an understanding of the coping strategies used by the stroke caregivers who had different socio-cultural and religious backgrounds from my study.

The numerous life changes faced by the family caregivers of stroke patients are wellrecognised. Coombs's (2007) phenomenological study that examined the experience of older caregivers as they cared for a spouse who survived a stroke revealed six interrelated themes. These themes were "experiencing a profound sense of loss, adjusting to a new relationship, taking on new responsibilities, feeling the demands of care, having to depend on the support of others, and maintaining hope and optimism" (Coombs, p. 116). The profound loss experienced by the caregivers is related to the loss of marital relationship a couple shared before the stroke occurrence. Besides this loss, participants also experienced a loss of leisure time and freedom. These losses greatly affected many aspects of the caregivers' life. Therefore spouse caregivers need to adjust to a new relationship with their stroke partner. This supports the earlier study that reported that the experience of loss of emotional intimacy was a significant issue among women caring for their stroke partners (O'Connell et al., 2001). The experience of loss faced by the caregivers as the consequences of the caregiving have been reported in many other studies (Anderson et al., 1995; Bugge et al., 1999; Forsberg-Warleby et al., 2002; Grant \& Davis, 1997; Johnson, 1998; Robinson-Smith \& Maloney, 1995). As a multidimensional experience, those losses vary and include disruptions or restrictions in 
leisure time and social activities, lack of freedom and personal time and inability to travel.

Although the location of all research studies was not revealed, most of what is known from these studies comes from the western perspective. It is necessary to explore the experience and the health needs of stroke patients and their caregivers from the Malaysian context to supplement the knowledge base.

\section{The needs of stroke patients and family caregivers}

\section{Rehabilitation needs of stroke patients}

Evidence suggests that although stroke is a common and serious condition, the management of stroke still relies much on rehabilitation intervention due to the unavailability of curative treatment for stroke (Langhorne \& Legg, 2003). Stroke patients need to commence rehabilitation therapy as soon as their medical condition is stable. Therapy could be terminated once it no longer produced any positive effects (Pollack \& Disler, 2002). However, there are some exceptional cases whereby "late" functional improvements were reported on some patients even two years after the onset of stroke (Pollack \& Disler). As the effects of stroke vary greatly between individual patients, careful selection of patients for rehabilitation is very important to ensure patients were received the most benefit from rehabilitation.

Talbot et al. (2004) examined the rehabilitation needs after stroke using focus group discussions among patients, caregivers, healthcare providers and administrators. Their findings showed that the most important needs related to rehabilitation expressed by the patients were to be accepted and have access to outpatient services for occupational and rehabilitation therapy. In addition, one of the suggestions from the healthcare providers group was to develop a stroke team based on a multidisciplinary approach. In addition, strategies should be implemented to improve and promote better communication between hospitals and community service. 
Evidence clearly shows that stroke patients are better managed by a specialised stroke team within the stroke unit (Cadilhac et al., 2004; Langhorne \& Legg, 2003; William, 2005). It is unclear what rehabilitation services are provided for stroke patients in Malaysia particularly after hospitalisation. This is an area of concern because most rehabilitation occurs in the community. It is worth examining whether they are facing the same problems as found in other countries in the literature.

\section{Support needs of stroke patients}

Much has been written in the literature in relation to the positive outcome of support services on stroke patients and their caregivers. Such positive outcomes include improved quality of life, enhanced and facilitated recovery and rehabilitation as well as community reintegration. The support needed by stroke patients is more significant during the early stage of recovery as the optimum recovery for stroke patients occur within three weeks to six months post-stroke (Pennington \& Burry, 1990). Support services needed by the stroke patients include family support, rehabilitation support in term of physical therapy, occupational therapy, speech therapy and access to the community resources. Most need support from the health professionals, for example, some stroke patients with aphasia require aphasia therapy. Aphasia therapy is proved to benefit stroke patients with aphasia in terms of increasing their quality of life and communication (Van der Gaag et al., 2005) and can be provided by expert professionals. They also need support in terms of the financial, physical, emotional and psychosocial aspects (Venketsubramaniam, 1999).

One of the aims of stroke management is to facilitate community reintegration (Mayo et al., 2000). Therefore community nurses need to expand their role to support and assist stroke patients and family to cope with the aftermath of stroke (Gibbon, 1994). Coping skills need to be developed among stroke patients and their families which emphasise the emotional and social recovery as much as physical recovery (Snape \& Burton, 2002). These will make life more meaningful for them. Another aspect of support needed by stroke patients and caregivers is related to sexual needs of stroke patients and their 
spouses. Approximately half of the participants in the Korpelainen, et al. (1999) study suggested the support needed was for sexual counselling after stroke.

Another issue that is still debated among researchers related to the effectiveness of rehabilitation provided for stroke patients. Dorcey and Vaca (1998) indicated that the rehabilitation of stroke patients at home produced better outcomes than in the rehabilitation centre. However, this might increase the burden of caring for caregivers or other family members as discussed previously. In comparison with the study by Hopman and Verner (2003) indicated that stroke patients were declining in recovery six months after being discharged from the rehabilitation centre. These different study findings possibly depend on the severity of the stroke.

Therefore the caregivers and healthcare community play a major role to reintegrate stroke patients in the community. For stroke patients, being at home is an important factor for recovery (Olofsson et al., 2005). This is due to the fact that they were living within their families and with a familiar environment. Therefore healthcare providers should identify and utilise factors that promote optimum recovery. The provision of psychological, emotional, social and physical support may help them with recovery and assist for internal adaptation.

Another area of study related to the experience of stroke patients concerns their attempt to return to work following stroke. Returning to work is considered one of the factors that facilitate or enhance the community reintegration of people with disabilities (Koch et al., 2005). As mentioned, more stroke patients survive with some residual disabilities after stroke. This phenomenon might lead to employment changes due to the impact of stroke on cognitive ability and skills related to their previous job. The Koch et al. study involved 12 stroke survivors and 13 of their primary caregivers. The study was guided by a grounded theory qualitative research design to explore the experience of right hemisphere stroke survivors in attempting to reintegrate into the community. Data were collected using interviews, demographic questionnaires and field notes. The findings 
demonstrated that many of them experienced weaknesses and fatigue which hindered them from resuming employment.

Currently, many studies indicate that there is an increasing awareness and understanding among healthcare professionals and the public that the recovery of the functional ability of stroke patients is not solely to determine a higher quality of life (Dorcey \& Vaca, 1998). Sometimes the perception of successful recovery or rehabilitation is different between the healthcare providers and stroke patients as demonstrated by Hafteinsdottir and Grypdonck (1997) in a review of literature in relation to stroke patients' experiences. It was found that stroke patients viewed recovery as the ability to return to pre-stroke life, not only the restoration of lost functions. A similar finding was revealed in the population-based study conducted by Jonsson et al. (2005). In this study, between 4 and 16 months after stroke, stroke patients' quality of life (QOL) scores were improved in the socio-emotional and mental Short Form Questionnaire 36 domains although the physical function domain scores declined. In addition, it was found that depression was the chief determinant of QOL. From the clinical implications, it can be suggested that there is an on-going need for intervention strategies to improve the QOL of stroke patients despite the decline in physical abilities.

While literature indicated the various supports needed by stroke patients and families, a study by Lutz et al. (2007) demonstrated that support in the form of care coordination/home-telehealth can provide a link to the healthcare system that they can contacted at any time when needed.

\section{Support needs of caregivers}

Stroke survivors' and their caregivers' need for support, such as social, emotional or physical as well as financial assistance, are widely discussed in the literature. Such supports will help to improve their quality of life and reduce the burden of caring among the caregivers. For example, support from other family members has been found to be associated with higher quality of life of the caregivers (Mant et al., 2000). Support services from the community such as stroke club, financial benefits or other community 
activities might contribute to the improvement of carers' well being. Moreover, much has been written about the importance of support from the nurses during hospitalisation. Nurses were encouraged to involve caregivers in the discharge planning process from the early stage (Bakas et al., 2002). This involvement would give great benefit to the caregivers as well as enhance recovery process.

In contrast, a study by Forster and Young (1996) did not show a better outcome in the intervention group of carers of stroke patients despite support from specialist nurses. One of the aims of this RCT was to evaluate whether specialist nurse visits (supports) alleviated stress in carers. Although the proportion of carers with stress decreased in both groups, there was no statistically significant difference in the stress level among carers between the intervention and control group. It is widely discussed that stroke survivors and caregivers need support from a variety of sources, but the types and quality of support needs to be taken into consideration. A factor that might influence the unfavourable outcome of this study was the use of quantitative measurement of the data. The combination of quantitative and qualitative measurement of the data might reveal a more reliable result.

Apart from the greatest need for information among caregivers, training in essential skills is proved to reduce the burden of care and contribute to improved patient outcome. Kalra and colleagues (2004) studied 267 patients and their caregivers with the aim to establish the impact of caregivers' training on patient outcomes and economic aspects of stroke care. Stroke patients were assigned randomly to receive conventional care or conventional care plus caregivers' training. This study demonstrated that caregivers' training reduced the burden and improved the quality of life of patients and caregivers without any adverse effect on patients' outcomes.

In comparison with the study by Forster and Young (1996), the above study showed that formal training for caregivers has a more positive impact on caregivers and stroke patients than specialist nurse visits. However, as mentioned earlier, a few other factors might influence the findings such as the level of functional disabilities of stroke patients 
and the quality and quantity of intervention. Therefore it is critical to understand the problems faced by caregivers in caring for stroke patients because a number of studies have found that it will influence the recovery process (Bhogal et al., 2003b; Clarke, 2002).

On the whole, it is therefore necessary for any research related to stroke patients to include caregivers or families as well because they are central to supporting survivors in the community. Disability in an individual family member affects the entire family system. Families have to adjust to the physical, psychological and emotional consequences of stroke. Therefore attention also has to be given to explore the phenomena faced by the caregivers of stroke patients.

Many studies have been undertaken relating to the effects of stroke on stroke victims and caregivers in other countries but to my knowledge, there is a limited study done from the nursing perspective in Malaysia. It is worth examining how the information and health education needs post-stroke of the Malaysian stroke patients and their families are met.

To summarise, to promote and facilitate optimum recovery, healthcare professionals, family caregivers and public should be aware of the support needed by stroke patients. On-going supports in terms of physical, psychological, emotional and social from the onset of stroke till the rehabilitation stage in the community are essential for successful rehabilitation.

\section{Studies on the information and education needs of stroke patients and caregivers The information and education needs of the caregivers}

Many studies were conducted to explore and gain an understanding of the needs of family caregivers in caring for stroke patients. Some studies found that the family caregivers' needs change or evolve over time due to the changes of stroke patients' health status (Brereton \& Nolan, 2000). One of the critical needs is for information related to stroke. 
Evidence to date demonstrates that there is a great interest among the family caregivers to know about stroke and stroke care. This is more obvious among the new caregivers. As mentioned earlier, with the advancement in stroke care, many more stroke patients survive. However, the majority of them are left with disabilities. Coupled with the current healthcare policy to reduce cost, most of them are discharged early to the community with some residual disabilities. One of the implications of this is that healthcare providers have less time to prepare the family caregivers for the caring roles (Pierce, Finn et al., 2004). The need of family caregivers of stroke patients in relation to the caregiving roles is widely debated in the literature (Brereton, 1997; Walling, 2005). For example, Brereton and Nolan (2002) studied the information needs of caregivers using an in-depth semi-structured interview guide involving seven caregivers with two to four years experience in caring for stroke patients. It revealed that the information needs were surrounding the issues of stroke and stroke impacts. Their key findings showed that healthcare providers should provide sufficient information on every aspect of stroke based on an individual's needs. This is congruent with the findings from a randomised control trial (RCT) undertaken to examine the effectiveness of early hospital discharge compared with early rehabilitation scheme for patients with acute stroke (Anderson et al., 2000). It revealed that both the intervention and control groups of stroke patients had the lowest level of satisfaction in relation to information and understanding of stroke.

Caregivers also found difficulty in getting information about patients' medical condition (Addington-Hall, 1995). This then led to dissatisfaction with the provision of health service. Similarly O'Connell et al. (2003) found that the information needs of the caregivers in the acute hospital setting and community setting were not being met or only partially met. Some of the needs are for information, social or psychological support as well as the financial support.

Hare et al. (2006) conducted a study of six caregivers and 27 stroke patients in Birmingham, England to explore the needs of stroke patients and their families. Data were collected through six focus group interviews. Two interview sessions were conducted for each focus group. The data were analysed using a constant comparative 
method. Three major themes emerged from the study: lack of information, having emotional and psychological problems, and the use of primary care as the first contact for information or problems. From all the groups and interviews, it was found that the participants were lacking information about all aspects of stroke and stroke services.

The above findings are consistent with those from a study conducted by Wiles et al. (1998). Wiles and colleagues undertook 31 in-depth interviews, 10 joint interviews between patients and carers, two sessions of interviews with carers and nine sessions with patients. The need for information was divided into three main areas: clinical information, practical information and service or resources in the community. The clinical information needs were related to information about treatment, recovery and prognosis of stroke, whereas practical information related to practical tasks such as personal care and social activities. Both studies demonstrated the need for information related to stroke and stroke care and availability of community services for stroke patients.

Pound et al. (1999) found that six months after discharge, caregivers expressed the greatest dissatisfaction with the provision of information on allowances and services. Furthermore, a study aimed to examine stroke caregivers' needs was conducted by Bakas et al. (2002) and involved eight African American and six white stroke caregivers at six months post-stroke. Their findings revealed the need for general information ranging from the warning signs of stroke, management of stroke related symptoms and complications and life changes post-stroke. Findings also indicate that caregivers needed to be educated prior to hospital discharge. Therefore it is very important to be aware of the information needs of the caregivers of stroke patients which will have a great influence in reducing the burden of caring as well as to improve their quality of life.

Many published studies on the caregivers' needs were carried out based in a western setting. However, similar findings were revealed in a study among Asian stroke caregivers. Jullamate et al. (2006) study of Thai stroke caregivers found that caregivers needed information on stroke and related issues as well as care giving activities at home. 
Stroke-related issues included prevention of recurrence, consequences of a stroke, strategies to perform ADLs and rehabilitation, care problems, available community resources and financial affairs. Lack of information given in the acute setting and prior to discharge leads to feeling unprepared for the caregiving role.

Although most studies focused on the negative impact of stroke on stroke survivors and caregivers some studies have revealed that in spite of living with a person with disability, caregivers were satisfied with their lives and had positive feelings about their care giving roles (Bluvol \& Gilboe, 2004). Hence it is worth exploring the factors that contribute to these positive feelings, especially in Malaysia.

\section{The information and education needs of patients and the caregivers}

Patient education is a major professional responsibility of nurses and a crucial part of nursing activities (Rausch \& Turkoski, 1999) and health education is a continuous need during patients' recovery (Denby \& Harvey, 2003). Furthermore, the importance of continuing health education is more apparent in chronic illnesses which require longterm rehabilitation such as stroke. As Eames et al. (2008) indicated, it is important to provide information effectively as it is an integral part of post-stroke care. However, there are persistent issues discussed in the literature that the information or education needs of stroke patients are not being met.

It has been mentioned earlier that the sudden occurrence of stroke has a great impact on stroke patients and their family members. Family caregivers are found to be lacking in preparation for the care-giving roles. In addition, many studies have demonstrated that the information needs of stroke patients and their informal carers in the hospital and after discharge are not being met and there is a dissatisfaction with the information provided (Eames et al., 2003; Hanger et al., 1998; Hanger \& Mulley, 1993). The areas of information needed during the acute stroke period are, for example, related to stroke, stroke effects, stroke causes, stroke prevention, recovery, treatment and prognosis (Eames et al.; Hanger et al.; Knight et al., 2006; Wellwood et al., 1994; Wiles et al., 
1998). The majority expressed a lack of understanding about the nature of stroke, the impacts and its causes (Knight et al.).

Other studies revealed that between $45 \%$ and $49 \%$ of stroke survivors and caregivers were not satisfied with the information received about stroke before discharge (Rodgers et al., 1999; Wellwood et al., 1994). Concerning these issues, a few factors need to be taken into consideration to find out whether their dissatisfaction was caused by a lack of information, inappropriate information, timing or method of the delivery of information. O'Connell et al. (2003) suggested that the education provided for stroke patients and their caregivers should be specific to their individual needs. This is due to the fact that the information needs of the patients may change over time.

The literature and my experiences clearly show the information needs of stroke patients also relate to self-care management, rehabilitation and available resources and support for them. Informal or family caregivers as well as stroke patients have a persistent need for information. Information on medication, recovery, illness and prognosis are among the areas of concern (Olofsson et al., 2005). These are similar to the findings from Venketsubramaniam (1999) on the overview of stroke patients in Singapore. The information needs included stroke symptoms, stroke risk factors, early treatments and screening programme.

The majority of stroke patients and their caregivers are not satisfied with the amount and quality of information provided (Hanger et al., 1998; Wachters-Kaufmann, 2005; Wellwood et al., 1994; Wiles et al., 1998; Yoon \& Byles, 2002). Evidence to date suggests that stroke patients, family members and the general public lack the knowledge related to stroke. Some stroke patients still need information related to stroke even two years after stroke although the types and focus of unanswered questions change over time (Hanger et al., 1998).

Many studies revealed that stroke patients and their caregivers did not receive enough information during their stay in the hospital. In a study by Yoon and Byles (2002), 
stroke patients reported a lack of information from the healthcare providers during hospitalisation. This qualitative study aimed to gain an insight into people's thoughts about stroke and assist in the development of educational resources in the community. Data were collected through a focus group discussion among 14 stroke patients, 10 carers, and 11 from the general public in New South Wales, Australia. This study also indicated that a lack of knowledge related to stroke is among the factors that cause delay in seeking for treatment. It implies the importance of stroke education. Nurses and other healthcare providers should provide comprehensive stroke education such as related to stroke signs and symptoms, the consequences of delaying treatment as well as how to adapt with the residual disabilities. This awareness and knowledge will help to reduce the consequences of stroke.

Apart from basic information about stroke, Hanger and Mulley (1993) also indicated that patients needed information about speech disorders caused by stroke and available community support. The basic questions related to stroke such as the nature and the causes of stroke as well as the relationship between stress or overwork and stroke.

Based on the literature, it is found that a lack of information and knowledge among stroke patients and their families may contribute to various negative consequences. Some of them are delayed presentation to hospitals, ineffective rehabilitation and slow recovery due to reduce motivation, non-compliance to treatment and reduced quality of life. Lott et al. (1999) revealed that a lack of knowledge of the warning signs and symptoms of stroke caused delay of presentation at hospital which in turn delays the initiation of treatment.

Current evidence in relation to treatment and care of stroke patients suggest that stroke patients should receive immediate treatment and care to minimise the consequences of stroke. A delay is when the time of arrival at hospital is more than three hours after the awareness of symptoms (William, 1997). As found in the study of 67 stroke patient in Indianapolis, less than one quarter of the stroke patients arrived at the emergency department within three hours of the onset of symptoms (William, 1997). Three hours 
from the onset of stroke symptom is critical for the effective treatment of stroke. Similarly, the finding from a large study in Denmark by indicated that only $25 \%$ of 1117 stroke patients arrived within 3.5 hours (Jorgensen et al., 1996).

Surprisingly, it was also reported that the delay in getting immediate treatment had been experienced by patients who had a stroke while in the hospital (Lott et al., 1999). In addition, stroke patients who had recurrent stroke still delayed getting medical attention (William, 1997). Apart from the reason that stroke symptoms may be painless, the other explanation might be the lack of knowledge of the need for immediate treatment for stroke. Another reason is the lack of knowledge of the signs and symptoms of stroke where the stroke patients wasted their time for hours before getting help (Lott et al.). These situations become a barrier for stroke patients to get to hospital immediately (Broderick, 2004). Even the healthcare professional themselves did not realise the need for an immediate care and treatment for stroke patients (Lot et al., 1999). Therefore it is suggested that comprehensive stroke education is needed to promote awareness among the public and health professionals of the importance of immediate care for stroke patients. This will reduce the delay in the presentation and referral of stroke patients. Current evidence shows that stroke patients should be given treatment within three hours of the onset of stroke to prevent extensive brain damage which takes a few minutes to hours to occur (Lott et al.). However, this advanced practice needs very skilful and experienced neurologists.

Improved knowledge among the public may, however, raise a few other issues for attention such as a high demand for a higher quality of health service. A randomised controlled trial (RCT) study conducted by Rodgers et al. (1999) revealed that improved knowledge among the family caregivers of stroke patients led to a reduction of their social function. It was assumed that it might be due to an increased awareness among the caregivers to spend more time with patients which then led to less time to socialise. The other consequence is more demand may be placed on the healthcare service in terms of quality of service, staffing and facilities. 
As mentioned, due to shortened hospital stay, family members have no time to prepare for the care giving-role. They have to take on the care-giving roles without adequate preparation in terms of knowledge and skills. Therefore the provision of education related to stroke care is needed. Even patients with minor stroke have difficulties in maintaining and regaining independence, re-establishing their sense of self and managing a marital relationship (Green \& King, 2007). As minor stroke patients may be discharged early, often less than two or three days following admission, nurses may need to provide the information or intervention. Green and King suggest that nurses should provide focussed counselling, taking consideration of the ageing process. Apart from that, the general population also have low levels of knowledge of stroke warning signs and risk factors (Kothari et al., 1997; Pancioli et al., 1997). However, a few studies demonstrated that the provision of health information does not result in an overall improvement in health status and psychological outcome of stroke patients (Rodgers et al., 1999). Concerning this, it may raise questions on the quality of information, the appropriateness and methods of delivery.

Based on the above information, action has to be directed to the development of a comprehensive education resource to address stroke patients' and caregivers' needs. There is a clear need to educate stroke survivors and caregivers, the public and other health disciplines in order to improve knowledge and skills in stroke care. It is also very important to promote an awareness of the immediate action needed during the onset of stroke. The findings from the literature on the educational needs of patients and families served as a basis for my study in exploring the educational needs of stroke patients and their family caregivers in Malaysia.

It is hoped that the development of the education resources can be used to guide clinical practice especially with nursing staff, other health disciplines and the public in particular those who are involved in caring for stroke patients in Malaysia. Based on the current evidence of stroke care globally and regionally, the need for education resources for stroke patients and caregivers in the Malaysian setting is highly recommended. 
In general, the decision for hospital discharge and the need for community service provision are based on the status of physical recovery (Foster \& Young 1996). In addition, the other risk factors such as hypertension need to be stabilised before discharge. After discharge, patients will usually be sent to their home to be looked after by family members.

A wealth of evidence concerning the information needs of stroke patients and families demonstrated that healthcare providers should provide accurate and sufficient information and this should be given as a routine based on the individual needs and illnesses. It should cover all aspects of the care given, treatment, prognosis, follow-up care as well as rehabilitation services. The information should be given orally and in writing with simple and understandable language. It is essential that it is emphasised many times during hospitalisation as well as after discharge (Denby \& Harvey, 2003; Eames, 2008; Garret \& Cowdell, 2005).

However, it also raises an issue of how to provide the most effective information and education for patients and their caregivers. In relation to this, systematic reviews conducted by Bhogal et al. (2003a) on 10 studies on family education intervention revealed strong evidence (four positive and one negative RCT with some positive findings) of the positive benefit of family education intervention using an active educational counselling approach. Besides that, it is also a quite surprising finding that there is strong evidence that an information package and workbook do not alter outcomes in terms of family functioning and outcome of stroke patients. An Australian study suggest that a multidisciplinary stroke team should develop a system that facilitates communication among team members and documentation, provision of quality written education material and verbal reinforcement of information provided for patients and their families (Hoffmann et al., 2007).

The association of health education needs and physical injury was revealed in Hinojosa and Rittman's (2009) study. They conducted a study to examine the association between health education needs and physical injury sustained as a result of caregiving activities 
which involved 276 stroke caregivers. Their findings revealed that caregivers with greater educational needs were almost twice as likely to have incurred an injury related to caregiving activities. Therefore provision of education in the form of knowledge and skills, for example skills related to lifting, moving, and positioning and handling may help to reduce caregivers' injury. This is an important finding and provides insight on how the lack of education might cause physical injury to family caregivers. Further, it is an area understudied in Malaysia.

\section{Key limitations of the review}

From the literature, it has been found that either qualitative and quantitative research approaches or a combination of them are used in studies related to the experience of stroke patient and their family caregivers. Hence, in order to explore the whole experience of stroke recovery and rehabilitation, there is a need to investigate the stroke patient's health journey through longitudinal studies. However, as reviewed by Hafteinsdottir and Grypdonck (1997), most of the studies evaluated stroke patients' health journey at 3,6 or 13 months from the onset of stroke. These may lead to a lack of detailed exploration and description of the whole process of recovery of stroke patients. On the other hand, these limitations have been reduced by using open-ended in-depth interviews.

The other critical factor in obtaining thorough knowledge of the experience of stroke patients is through their point of view. However, this area has received less attention (Hafteinsdottir \& Grypdonck, 1997). This might be due to some stroke patients being very ill or in a coma and having problems with communication which prevent them from being involving in the research. The reviews from the literature showed that most of them were excluded from the published studies.

\section{Conclusion}

This chapter has highlighted various major issues surrounding post stroke rehabilitation. The review of the literature has revealed the devastating effects caused by stroke on patients as well as their family caregivers. They have to face various kinds of impacts of 
stroke in terms of physical, psychological, and psychosocial factors. Moreover, the critical analysis of the studies on stroke patients and caregivers also has demonstrated that stroke patients and family caregivers have multiple and continuous needs for information and education during the recovery period related to stroke and post-stroke care. The area of information needed include the nature, impact and causes of stroke, warning signs of stroke, prevention of stroke recurrence, recovery, treatment and prognosis, managing co-morbid condition and management at home. Lack of knowledge and information following stroke may lead to various negative consequences to patients and families. These might influence the recovery and rehabilitation process.

The literature also showed that stroke patients and caregivers need a range of supports to facilitate and optimise recovery. This extensive review of the literature identified the gap addressed in this study and informed the development of the study framework. The framework influenced the choice of research methodology.

The review of the literature also showed the common limitations found in the studies in the examination of the health needs of stroke patients. One of the common impacts of stroke is the inability to communicate; hence, some of the study findings might not represent the health needs of the stroke patients with communication difficulties. This study therefore included stroke patients who could communicate their needs.

The next chapter will present the study framework of the "Stroke Care Community Model" (SCCM) which served as a guide for this study. This theoretical framework informed the design of the study in various ways such as the development of two phase study design, interview guide and the selection of study participants. 


\section{Chapter 4: Theoretical framework}

\section{Introduction}

In the literature review, the resource needs of the home-based stroke patients and their family caregivers were presented. The needs of patients and families post-stroke include educational needs related to skills, information following stroke and information about resources and support available. Most of the study findings come from a western perspective. In this chapter, a theoretical model of the home-based stroke care is presented. The theoretical perspective that was developed to guide this research was the Stroke Care Community Model (SCCM). This model was adapted from the Model of Chronic Care by Wagner and colleagues (Wagner et al., 2001) using insights gained from the research findings discussed in the literature. The SCCM emphasis is on the need for stroke patients and families to be informed and prepared, which is the primary interest of this study. The model was developed with careful consideration to suit the Malaysian healthcare system, community infrastructure and societal values.

\section{The Wagner's Chronic Care Model (The Wagner's CCM)}

Wagner's model is an internationally accepted model on chronic disease. There are six fundamental elements that form the basis for the Wagner's CCM: the community, the health system, self-management support, decision support, delivery system design and clinical information system (Wagner et al., 2001). The idea that underpins the model is that the present healthcare system is a barrier to effective management of chronic conditions. Wagner's CCM was developed by Ed Wagner and colleagues at the MacColl Institute for Healthcare Innovation in Seattle, Washington State, in the 1990s (Mcevoy \& Barnes, 2007). Their model is an approach to support high quality and effective management of chronic conditions to complement our current healthcare system that focused on acute and episodic conditions (Wagner, Austin \& Korff, 1996; Wagner, Davis, Schaefer, Korff \& Austin, 1999). Studies related to the implementation of Wagner's CCM have been done in various health settings (Glasgow, Orleans, Wagner, Curry \& Solberg, 2001; Hung et al., 2007; Mcevoy \& Barnes, 2007; Solberg et al., 2006). These studies demonstrated that the Wagner's model not only served as the framework 
for managing existing chronic condition but could also be used as a blueprint for preventive care. However, the majority of the studies focused on chronic diseases such as diabetes, asthma and depression which have very different trajectories to stroke.

From the review of the literature, it is found that the component of self-management in Wagner's CCM is more focused on the patients themselves as care managers with less emphasis on families (Glasgow et al., 2001). This might be due to the fact that patients with chronic conditions studied are independent and have good levels of self-care. However, it is argued that this approach is not appropriate with some chronic condition such as stroke. This is in part due to the nature of the impact of stroke on the stroke sufferer and their families. The majority of stroke patients live with some residual disability after the stroke. The disabilities faced by stroke patients will affect their ability to perform some of the activities of daily living (ADL) such as dressing, hygiene, mobility and feeding. In such cases, the families will play a very important role as caregivers for stroke patients. Some stroke patients are severely affected by stroke and dependent on their caregivers for daily activities. In comparison with other chronic diseases such as diabetes and asthma, the roles of the family in stroke care are much more significant.

The SCCM emphasises the patient and family. It recognised the need for a care delivery model to support high quality care for the home-based Malaysian stroke patients and their family members. Within the Malaysian context, there is limited knowledge on how to maximise stroke recovery and the quality of life of the stroke patients and family caregivers and minimise complications. Therefore it is believed that there is a need for information and knowledge, skills, supports and resources for the home-based Malaysian stroke patients and family caregivers. This is also because most of the on-going care for stroke patients is given in the community by the family. The continuity of care provided by families has also been highlighted in studies in developed countries (Knight et al., 2006; McBride et al., 2004). 
The SCCM is an appropriate model and concrete guide for this research as it focuses on the educational needs for information and knowledge following stroke, skills, and support and resources available for patients and families post-stroke. This is also to ensure the provision of a higher quality of health care for stroke patients and families in the community. The model focuses on the stroke patients and their families in the community, as shown at the centre of the model (Figure 5). Wagner's model emphasises the role of community and the health system. It also focuses on preventing acute episodes of illness, management and prevention of symptoms and medications in chronic diseases (Bodenheimer et al., 2002) and does not have sufficient emphasis on recovery or the rehabilitation process, whereas the focus of SCCM is on achieving optimum recovery after stroke. However, aspects of the Wagner's CCM are useful because of the recognition of the role of the community, the self-help component and interaction with health service as well as decision-making components. The SCCM focuses on achieving optimum recovery and rehabilitation, prevention of stroke recurrence and complications and promoting functional abilities and independence as well as adaptation to life-style changes after stroke.

\section{The theoretical model - SCCM}

In the present context of SCCM, the term 'stroke patients' refers to the discharged stroke patients and stroke patients living in the community. In the absence of a comprehensive rehabilitation service and a home-based nursing service the SCCM framework argues that stroke patients need to be fully informed and prepared to be involved in their health care. Furthermore, home-based stroke patients are strongly supported by informed and prepared family caregivers.

In the home-setting, the SCCM shows that stroke patients and families need education related to skills, information and knowledge, support and easy access to resources for successful recovery, minimising complications and maximising of quality of life. These four components form the basis of the model. The SCCM is located in a community and the model assumes the presence and involvement of the healthcare services. 


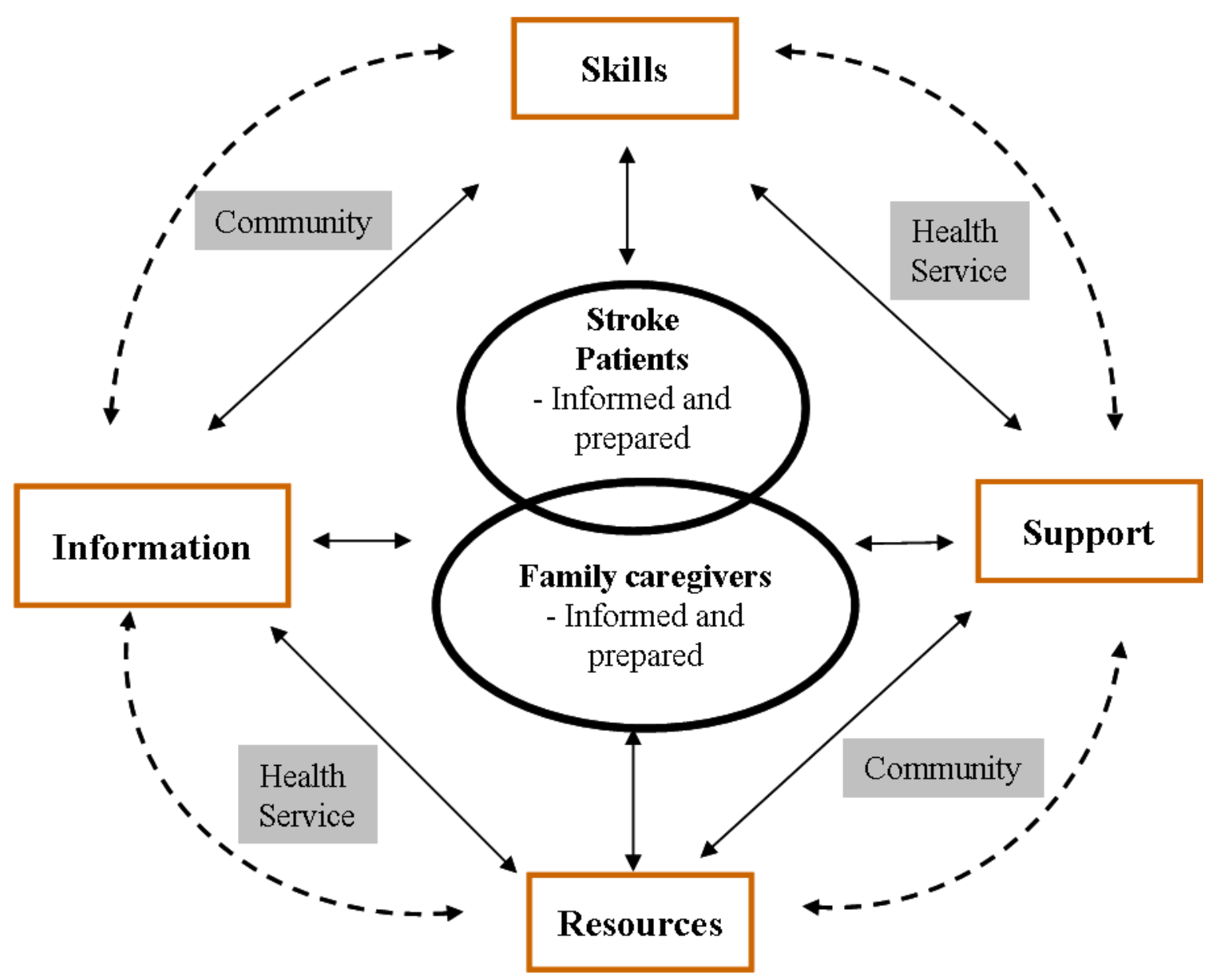

Figure 5: The Stroke Care Community Model (SCCM)

The SCCM recognises the family's contribution in the home due to the major role of families in providing on-going care for Malaysian stroke patients in the community. Therefore the approach of care in SCCM focuses on self and family management as well as recognising their cultural practice and religious values in post-stroke care. This is congruent with Korff et al. (1997) who indicate that patients and families are the main providers in chronic care. The SCCM was developed to suit Malaysian stroke patients and the importance of the family role in the home setting. Unlike Wagner's model, limited emphasis is placed on the health system in the SCCM model. This is because the implementation of SCCM in the Malaysian setting needs to match the current Malaysian health care system, availability of community resources, stroke rehabilitation structure, 
living arrangement of elderly people and cultural and religious values. Stroke rehabilitation in Malaysia needs to emphasise self and family management.

In the current practice of home-based stroke care in Malaysia, community health nurses play a major role in supporting stroke patients and their family caregivers at home. They should be able to identify the relevant individualised needs of stroke patients and family caregivers and implement interventions appropriately. Although stroke patients and families are core in the SCCM framework, it emphasises the need for support through collaborative management with healthcare providers.

In the context of the Malaysian stroke patients and their family caregivers, the SCCM provides four essential components for successful rehabilitation and recovery: skills, information, support and resources. The literature shows the need for these four components for effective stroke care. This is consistent with the findings of the studies in developed countries that demonstrate that stroke patients and families need knowledge, skill, support and resources in order to achieve successful recovery. However, given the major role of the family in stroke recovery at home setting, it is assumed that family values may influence the process of recovery. All the elements in SCCM are connected by double arrows to show the interrelated and interdependent relationship between each component in the SCCM.

\section{Components of the SCCM}

The four components of SCCM are discussed under the following subheadings: skills, information, support and resources.

\section{Skills}

Skills refer to the activities which require special training and knowledge. Jester (2007) indicated that skills in stroke care refer to the performance of activities to aid positive neuromuscular plasticity and recovery. Various kinds of skills are needed to achieve optimum recovery and rehabilitation. Such skills comprise self-care, mobility, 
communication, social skills, adaptation and coping skills, exercise, and occupational skills.

There is much evidence for the effects of stroke on the ability to perform activities of daily living. The literature showed that $50 \%$ of stroke patients will survive with some form of disability and need rehabilitation. The most common problem is related to performing ADL such as feeding, dressing, hygiene and mobility. In addition, the need for various kinds of rehabilitation such as physical therapies, occupational therapies and speech therapies are widely discussed in the literature. However, there is some evidence showing that rehabilitation therapies will most benefit the stroke patients with mild to moderate disabilities compared with those patients with severe disabilities. Therefore it is arguable whether the implementation of the SCCM will benefit the severe stroke patients.

The other common impact of stroke is related to swallowing abilities. The majority of stroke patients were found to have swallowing problems especially during the early stage post stroke (Smith, 2004). Family members need the skills and strategies on how to feed stroke patients who have swallowing difficulties, the appropriate position to be used during feeding and the types of food to prevent choking or aspiration. They should be able to recognise the warning signs of aspiration. A lack of skills and knowledge in this area may lead to various complications.

In addition, stroke patients and families need skills related to communication strategies. Speech therapies are needed for stroke patients with aphasia although it is found that spontaneous speech recovery may occur in some cases (Dobkin, 2005). Some studies have indicated that intensive speech therapy within a short period will result in positive outcomes for the stroke patients with aphasia (Bhogal et al., 2003a). Family members need to be taught the various alternatives for communication and how to communicate effectively with their stroke family member with aphasia. The inability to communicate may have negative emotional and psychological effects on the stroke patients and 
families and reduce self-esteem. This will also affect the recovery and rehabilitation process due to reduced motivation.

The skills and knowledge to detect and assess the improvement or deterioration of stroke patients' condition are equally important. This will allow for early detection of possible complications and prevent stroke recurrence. Family members also need skills in how to minimise complications such as assisting with exercise, positioning, prevention of skin breakdown and contractures.

One of the goals of stroke rehabilitation is to facilitate community reintegration. Some of the strategies are through reemployment or returning to work. Therefore healthcare providers should teach occupational skills and help stroke patients to relearn the skills that are based on their maximum functional abilities, to facilitate or enhance community reintegration. This is also a means to develop social skills by interaction with people in the community.

With sufficient and appropriate knowledge and skills, stroke patients and families may have more opportunity for successful self-management and decision-making. As Coleman and Newton (2005) have indicated, self-management support is the core in chronic illness. The SCCM supports the proposition that self-management of stroke patients should be strongly supported by family members.

\section{Information}

Within the context of this study, the provision of information refers to health information. Knight et al. (2006, p. 83) defined the provision of health information "as a communicative exchange where people were given facts or explanations that contributed to their knowledge of their health condition. Health information may have been requested by the patient or given to the patient by a health professional."

The purpose of providing the information is to "inform, reduce anxiety, change behaviour and empower individuals and thereby prevent illness progression, 
complications or unnecessary handicaps" (Hanger \& Wilkinson, 2001, p. 113). The SCCM recognises that stroke patients and family members need information as part of best practice for home-based stroke care.

Many studies have demonstrated the need for information among stroke patients and their caregivers (Hare et al., 2006). The World Health Organisation declares that all patients have a right to receive information based on their needs that is accurate and factual and easy to understand, about their health condition (WHO, 2003). The National Stroke Foundation of Australia suggests that

all stroke survivors and their families should be provided with timely, up-to-date information in conjunction with opportunities to learn via education from members of the interdisciplinary team and other appropriate community service providers. (2005, p. 14)

The provision of information benefits the patients in terms of increased motivation, reduced anxiety, and improvement of compliance to treatment, increased satisfaction with healthcare, better adjustment to stroke, and empowerment of patients in their relationship with health professionals (Knight et al., 2006).

The SCCM emphasises that families need to be prepared to care for their stroke family members in terms of knowledge and skills. They need information about the resources available in the community and ways to access to these resources. Families also need guidance on what they should or could do and the possible complications which may occur. With good knowledge and skills, they can make the right decisions that will improve patients' recovery and minimise complications.

It is acknowledged that some studies have highlighted different perceptions between patients and health professionals in the area of information or education needed by the stroke patients (Choi-Kwon et al., 2005). Therefore the provision of education for stroke patients and caregivers should be based on an understanding of these differences and research evidence. In providing the education through written material, Hoffmann and McKenna (2006) suggest that the reading ability and informational needs of stroke 
patients and caregivers as well as the reading level, content and design characteristics of the material need to be taken into consideration.

The stroke patients and their families need information about rehabilitation, self-care management, medication and knowledge about stroke symptoms, prevention of stroke recurrence and stroke risk factors. Information about the availability of community resources may provide an opportunity for stroke patients and families to utilise these resources for successful recovery and rehabilitation.

With information and skills, stroke patients and families will be able to set up joint goals with the providers. For example, during occupational therapy, a stroke patient may aim to be able to hold a cup using her/his weak arm after three weeks of practice. Families should be able to choose suitable types of food for stroke patients with swallowing difficulties. Speech therapists may provide knowledge on the various means of communicating with aphasic patients. Besides that, knowledge and understanding of the differences between expressive and receptive aphasia will greatly benefit stroke patients with aphasia and their families.

However, the types of health information required by stroke patients in the acute setting are slightly different from those of post-discharge patients. In the acute setting the kinds of information needed include an explanation and feedback on tests and results, details of the prognosis, availability of future support and the nature of stroke (Knight et al., 2006).

A review of the literature revealed that the majority of the studies have excluded stroke patients with aphasia. This is due to the difficulties of obtaining data from the patients because of their impaired ability to communicate. Therefore it can be argued that the provision of information is beneficial for stroke patients with aphasia. Besides that, some studies have reported that less time is spent with stroke patients with aphasia when providing information and involve fewer topics compared with the stroke patients without aphasia (Knight et al., 2006). 


\section{Support}

The SCCM recognises the importance of support in many areas of stroke care for homebased patients and their caregivers. Support can be provided by the healthcare providers, families, community resources, voluntary organisations, stroke support groups and private practice. Many studies show that the burden of care faced by the stroke family caregivers impacts emotionally, physically, socially and financially and leads to reduced health status. Therefore stroke can be considered as a family illness. Given the burden faced by families in their caregiving role, the SCCM emphasises that the health care system and other support systems should consider families as well as patients. Evidence also shows that patients and their caregivers need support throughout the recovery and rehabilitation period (Mant et al., 2000; Mayo et al., 2000; Venketsubramaniam, 1999). This includes emotional, physical, psychosocial, financial and rehabilitation support.

Rehabilitation support services provided by the rehabilitation therapists comprise physical therapy, occupational therapy and speech therapy. Patients need support to socialise in the community to prevent social isolation and promote self-esteem, share experiences with other stroke patients and relearn skills based on their abilities. Mayo et al. (2000) have indicated that one of the major aims of stroke management is reintegration into society. Some of the strategies to socialise or reintegrate into the community are by providing support to return to previous employment or pre-stroke activities and involvement in community activities. The positive outcome of the support includes improvement of well-being, increase in quality of life, enhancement and facilitation of recovery and rehabilitation as well as community reintegration. Studies show that patients' perception of successful recovery is more on the ability to return to pre-stroke activities which may contradict the perceptions of the healthcare professionals (Hafteinsdottir \& Grypdonck, 1997). Healthcare professionals are more focused on the return of functional abilities of the stroke patients.

Therefore the overall goals of support for stroke patients and family caregivers is to move from a dependent state to independence and full functioning as much as possible and to improvement and higher quality of care. In the future it is hoped that the home- 
based stroke care in Malaysia will be supported by policies related to stroke patients' and caregivers' rights. Stroke patients and family caregivers need on-going support throughout the recovery period although the type and level of the support may decrease over time.

\section{Resources}

The SCCM framework recognises the importance of patients and families being supported to access any resources available throughout the stroke trajectory. This implies that education should be provided for patients and families so that patients and families are able to utilise those resources. Patients and families have the right to know about possible resources such as the availability of public and private rehabilitation services, stroke support groups, stroke association, various types of rehabilitation therapy, financial aid, other community resources and sources of information and education.

\section{Interface and relationship with the community}

The SCCM framework emphasises the role of the community in supporting patients and families along the stroke trajectory. One of the main goals of stroke rehabilitation is to reintegrate patients into the community (Bhogal et al., 2003b; Farzan, 1991). This might be in the form of providing an opportunity to be involved in community activities or easy access to any support services within the community. The literature revealed that patients most value being able to return to pre-stroke activities (Downswell, 2000; O’Connell et al., 2001). In addition, this would help in promoting socialisation, increasing self-esteem and motivation and facilitating adaptation. This is due to the nature of the effects of stroke, such as difficulty in communication and mobilising, which then lead to social isolation. Therefore the various forms of support from the community are essential for patients and families. From personal observation, I consider that religious groups or religious activities may benefit stroke patients in their rehabilitation. As an age-related disease, the majority of stroke patients are among the elderly whose religious values may influence their ability to adapt and cope with the life-changes after stroke, as well as acting as an emotional support. 


\section{Interface and relationship with the healthcare service}

The SCCM framework suggests that home-based stroke patients and family members need to be supported by a well-established and well-prepared healthcare team. The model assumes the involvement of the healthcare service in a particular community health service for home-based stroke care. A community health service or community rehabilitation team should comprise health professionals from the multidisciplinary team. These may include rehabilitation therapists, physician or neurologist, psychologist or counsellor, community nurses, nutritionist and social worker. Therefore a standard referral system should be developed to facilitate the process of transition from an acute care setting to the community setting.

In the present Malaysian healthcare system, a well-planned and standard referral system from institutional care to primary health care is needed in relation to post-stroke care. District hospitals, health centres and rural clinics should play a crucial role in providing health services for the stroke patients and family members at home-setting. Mobile clinics will also give great benefit for the stroke patients from the remote areas of Malaysia. Therefore in relation to Malaysian stroke patients, primary care should play an important role in providing care and support for home-based stroke patients. However, to date, there is no available data on the current practice of primary care services related to the stroke care. Current evidence is needed to give a true picture of the services provided. Even studies from the developed countries demonstrate that stroke patients are of the opinion that primary care is an important point of contact for stroke patients and their caregivers (Hare et al., 2006).

The theoretical framework presented underpins my research as it forms the basis of the selection of the research design and the aspects or issues that will be explored in the study. With this theoretical framework in mind, this study was planned in two phases. To reiterate, Phase 1 involved the identification of the educational needs of stroke patients and their families based on their perceptions and those of healthcare professionals. Phase 2 focused on obtaining health professionals' views on developing educational strategies based on the needs identified in Phase 1. 
The SCCM framework was developed to better suit the purpose of the study because it contains the four elements (skills, knowledge, support and resources) which are considered as integral aspects of home-based stroke patients and their families. It also contains the variables and relationships for the purposes of the research to be undertaken. It was assumed that the educational needs of patients and families would cover the four elements outlined in the theoretical framework. Therefore the instruments used to gather the data were able to achieve the aims of the study.

The framework informed my decision that the research needed to involve patients and family caregivers because these two groups would have some separate and some shared needs. The families' views on the need for education are also concurrent with the increasing involvement of families in post-stroke care in Malaysia. In addition, it is in line with the SCCM framework with its emphasis on the importance of families in managing patients at home. Therefore discharged stroke patients and their families who are at home needed to be involved. In addition, the SCCM framework incorporated community more than the Wagner's model. Due to the nature of stroke recovery and rehabilitation, the SCCM framework positioned that post-stroke care was largely delivered outside of the health services. As revealed in the literature, most stroke patients need ongoing care and treatment while in the community (Oloffson, 2005). Therefore it informed the decision that participants need to be recruited from those who were outside of the health service or in the community.

The SCCM framework recognised there is a role for the health services which informed the decision that health professionals who are directly involved in stroke care needed to be invited to participate. As stroke recovery and rehabilitation involves multidisciplinary team, health professionals from various disciplines were approached based on purposive sampling method.

The framework also informed the interviews. The framework identified that patients and families educational needs would most likely span four different elements: skills, 
information and knowledge, support and resources and these differed. Participants needed to be asked on those four elements. Guided by the framework, the interview guide was developed in such a way that the interviews would allow participants to talk freely of their educational needs around these four elements. The framework was particularly useful for Phase 1 of the study. The interviews were able to provide information on how patients and families were informed and prepared following stroke as shown in the model. The sudden life changes caused by the disability faced by patients after stroke shows that the need for patients and families to be informed and prepared is different compared to other chronic diseases such as diabetes, asthma and heart disease. Whereas, in Phase 2 only health professionals participants were involved due to the importance to obtaining their opinions and views on prioritising and developing educational strategies for patients and their families. What was unexpected however was that to achieve informed and prepared patients and families, the health service has an important role and that change overall would only happen at the health service level. This is consistent with the framework that health services need to be involved.

As mentioned, the framework focused more on patients and families in the community. The model of pulling out the community allows for all the material related to cultural and religious practices such as massage, prayer, and other practices to be revealed.

The theoretical framework also provides an insight on the analytic method. It was used as a boundary to limit the scope of the relevant data by focusing on specific issues for analysing and interpreting the data. The framework provided the lens to identifying themes based on the four elements in the model.

\section{Conclusion}

The theoretical model presented shows the relationship among the essential elements in home-based stroke rehabilitation. The model has four main components: skills, information resources and support. The conceptual framework shows the major issues the study focuses on. The research objectives will be addressed within the framework of 
the theoretical model. Given that education is needed on the issues of knowledge, skills, support and resources for stroke patients and their families following stroke, the SCCM framework is considered an appropriate framework to guide and position this thesis. This framework also guided my choices of the research design for this study, which is presented in the next chapter. 


\section{Chapter 5: Research design}

\section{Introduction}

This chapter outlines the methodology and the two stage research design of the study. It is divided into four main areas: the aims of the research, methodological strategy, overall features of the research design and features of each phase design. A detailed description of the aims, design and the processes of each phase are presented. Each phase also describes the basis for the selection of the participants and provides a brief description of the study setting. In regard to participants, it was intended from the beginning that five stroke patients, five family caregivers and approximately 12 health professionals would be involved. This number of patient and caregiver participants was considered sufficient to obtain detailed data on the focus of the study. As Kvale (1996) and Sandelowski (1995a) state, the number of study participants depends on the research methods as well as the availability of the eligible participants at that relevant time. In addition, more health professional participants were chosen to accommodate their multidisciplinary nature and to capture key members involved in stroke care within the study setting. The procedure and methods of the data collection and data analysis for each phase are described. This chapter concludes with the considerations of rigour and the ethical issues encountered for each phase of the study.

\section{The aims of the research}

The study aims were to identify and explore the educational needs of Malaysian homebased stroke patients (HBSP) and their family caregivers based on their perspectives and those of healthcare professionals. Further, the study aimed to explore the resources currently available for patients and families during the rehabilitative period and the existing practices of healthcare professionals in relation to education and health information provided to stroke patients and their family caregivers in their preparation for discharge. Finally, the study aimed to prioritise and develop an implementation plan for the development of health education resources that extended the existing practices. 


\section{Exploratory descriptive qualitative study}

This study focused on prioritising and developing strategies for health educational needs of HBSP and their family caregivers in Malaysia based on the research evidence. It is hoped that the education strategy will meet their needs within the local context of the Malaysian cultural and religious background. The nature of stroke rehabilitation involves the multidisciplinary team. The research was designed to answer the educational needs of Malaysian stroke patients and their families.

\section{Overall features of the research design}

This study involved two phases: Phase 1 - baseline data collection to identify areas for the education and resource development and to establish the current resources available and Phase 2 - working with the health professionals to prioritise the needs identified in Phase 1 and to develop an implementation plan or strategies for addressing educational needs.

The study employed a qualitative approach and used in-depth interviewing as the main data collection method. Sandelowski (2000) stated that language is a vehicle of communication in qualitative descriptive studies. This study is a qualitative descriptive study because straight description of the phenomena are desired based on the research objectives and research questions. This qualitative approach was appropriate for my study in order to obtain detailed data. As stated by Kvale (1996) the use of the qualitative interview allows people to express their opinion from their perspectives in their own words. With respect to this study, participants were encouraged to express their feelings, experience and opinions or suggestions freely and openly related to the topic of the study. In addition, face-to-face interviews using a semi-structured interview guide provided an opportunity for the researcher to obtain deeper and richer information related to sensitive issues. In the context of this study, as the consequences of stroke may affect the physical, psychological, emotional and cognitive ability of stroke sufferers, it was anticipated that some sensitive issues might have needed to be expressed during the interviews. 


\section{Phase 1 - baseline data collection}

Phase 1 aimed to establish the current resources available and the education resources needed by the HBSB and their family caregivers. This information was used to make recommendations about the educational resource needs based on the needs of the stroke patients and their family caregivers as well as health professionals. In the future it will be used to inform the development of resources. In this phase, data were gathered by face-to-face interviews using a semi-structured interview guide for various groups of participants. The details of the recruitment process are described further in the section on recruitment strategies. The interviews covered all the details about the focus of enquiry in the study (Appendix 1, $2 \& 3$ ). The interviews were conducted by the researcher alone and were recorded to ensure accuracy.

\section{Phase 2 - prioritising need and developing implementation plan}

Phase 2 commenced after the analyses of the data from Phase 1 were completed. Phase 2 involved a presentation of Phase 1 findings in focus groups and individual interviews with health professionals who were previously involved in Phase 1. Participants had to re-consent for the focus group and individual interviews. The purpose of Phase 2 was to prioritise and develop implementation plans for the development of health education resources. The data from this second phase were also recorded.

\section{Phase 1 of the study - design and process}

\section{Aims of Phase 1}

The main aim of Phase 1 of data collection was to gather information about the areas of education or information needed by HBSP and their family caregivers according to their perceptions and those of the healthcare professionals. Further, the aim was to identify the current practice in relation to the provision of information and education for stroke patients and their caregivers post-stroke.

\section{The study context}

All the study participants were identified from the Hospital University Sains Malaysia (HUSM) except one participant from the National Stroke Association of Malaysia 
(NASAM). The setting of HUSM, located in Kubang Kerian about five kilometres from Kota Bharu (the capital town of Kelantan), was purposely chosen as the research location. The selection was based on several factors. HUSM is a university teaching hospital and the only teaching hospital in Kelantan. HUSM was chosen as the study site to indirectly discover a general overview of the kind of service specifically related to educational activities provided for patients and families. Interviewing the patients, families and other health professionals was applied as the method of study as a way to learn the level of information and education received by patients and families. HUSM is assumed to be able to provide better education and information to patients and their families and this can be examined from this study. In addition the availability of facilities as well as the provision of quality healthcare service is better than other hospitals. Therefore the extent of satisfaction that patients and their family caregivers feel may indirectly assessed from this study.

Moreover, as a tertiary referral centre, HUSM receive cases from all over Malaysia especially from the east coast of the Malaysian Peninsular. In addition, since the establishment of the Neuroscience Unit in 2000 which was later upgraded to the Neuroscience Department, HUSM operates primarily as the central reference for all neurological and neurosurgical patients from the hospitals in the east coast of the Malaysian Peninsular. HUSM may be considered as representative of all the teaching hospitals in Malaysia due to the similarity in hospital management and nurses' background, qualifications and training as well as the standard of facilities provided. Apart from the State General Hospital located in Kota Bharu district, all stroke patients throughout Kelantan who require admission are usually admitted to this hospital. It is believed that as a teaching hospital it can be a model in terms of high quality of services and health education for all patients and their families as well as the public in general. The involvement of stroke patients, nurses and other health professionals from this hospital is an important contribution to this study. 


\section{Participants and recruitment strategy}

In Phase I of data collection, the study participants comprised a diverse group of health professionals, discharged stroke patients and family caregivers. Health professional participants included a speech therapist, occupational therapist, physiotherapist, academic nursing staff, registered nurse, nurse manager, medical doctor and a member from the national stroke association of Malaysia. Five stroke patients, five family caregivers and 12 health professionals were chosen as study participants. As this study employed a qualitative design using in-depth interviewing, five participants from each group of patients and families were considered sufficient to provide information on the focus of the study. More health professionals were recruited due to the importance of covering all the multidisciplinary groups of health professionals involved in post-stroke care and recovery.

The study participants were recruited based on a purposive sampling method and snowball sampling. According to Berg (2007), in developing a purposive sample, the researcher uses their special knowledge or expertise to recruit participants. As I came from the same working area, some of the health professional participants and their area of expertise were known to me. I chose some participants based on their expertise and others who were most representative for the issues in my research. This kind of sampling method was chosen because the participants were considered as the expert and knowledgeable because they have the first-hand experience related to the issues under study. As Coolican (2004) and Polit and Hungler (1993) indicate, participants should be able to provide information from their own experience or be knowledgeable about the focus of enquiry in the study. Moreover, this purposive sampling method is sometimes referred to as judgemental sampling due to the researcher's conscious selection of the participants to be included in the study (Burns \& Grove, 1997; Polit \& Hungler). This means that the study participants are judged to be the experts or the best persons who can provide the information in relation to the issues in the study even though they might not be representative of the population. However, all study participants should meet the inclusion criteria to be able to participate in the study. The stroke patients in the study 
were chosen from those who had been discharged from the hospital's medical wards, where all stroke cases are admitted.

\section{Recruitment strategy for family caregivers and stroke patients}

Before any contact with the hospital was made, ethics approval to conduct research in Malaysia was obtained from the Victoria University of Wellington Human Ethics Committee (Appendix 4). Then, a request to undertake the research was made to the HUSM hospital director (Appendix 5). Written approval from the director was obtained (Appendix 6). The letter addressed to the hospital director explained the objective of conducting the interview with the hospital staff and requested assistance of the hospital to provide the contact details of stroke patients who had been discharged from HUSM. The contact details of the stroke patients and their caregivers were obtained for those who were selected as potential participants.

In recruiting stroke patients and their family caregivers, it was not pre-determined whether family caregivers or stroke patients would be first recruited. However, from my personal experience, and experience during a pilot interview, I realised that communication mostly happens with the families. The baseline information on patients' conditions and health status was obtained from the families. In addition, all the contact numbers provided in stroke patients' records belong to the family members or patients' next of kin. It was therefore decided that to recruit patient and caregiver participants, the initial contact would be with families. Therefore caregiver participants were first identified from a stroke patient list. The list of all the stroke patients who were admitted into the hospital's two medical wards in 2007 was obtained from each ward registration record. Stroke patients were identified based on the diagnosis as recorded in the registration book. As the contact number of patients or significant others were rarely written in the ward registration book, contact numbers were obtained from computer data at the medical record office of the hospital. Family caregivers were first identified and recruited as they are the first contact person stated in the patients' records. It was assumed that any conversation or enquiry regarding a patient's condition would be made

with family caregivers. I intended to recruit $5 \mathrm{HBSP}$ and 5 family caregivers to be 
interviewed. Burns and Grove (1997) and Miles and Huberman (1994) stated that qualitative studies tend to use small sample sizes and employ semi-structured interviews to study the phenomenon in-depth. This is supported Holloway and Wheeler (1996) and Sandelowski (1986) who argued only small sample sizes are required because of the large volume of verbal data that must be analysed. In addition, Holloway and Wheeler (1996) also stressed that the number of participants depending on the type of research question, material and time resources, and the number of researchers involved.

The recruitment process commenced by contacting family caregivers using the contact number obtained from the stroke patients' records. The inclusion criteria for the family caregivers to be chosen as study participants were those who had some experience in caring for the stroke family members at home and who identified as primary caregivers. In order to obtain the family caregivers, the family caregiver of every $20^{\text {th }}$ stroke patient listed was contacted to identify the potential participant. For those who declined or did not meet the inclusion criteria, the next person on the list was approached. At the same time, during recruitment telephone conversation with families, the patient's eligibility was assessed based on the verbal report given by the families to identify whether they met the inclusion criteria to be a participant. This meant that the same recruitment procedure was applied for all stroke patients. The basis of exclusion for the family caregivers included their stroke family member having died soon after discharge, their declining to be involved, and having no experience in caring for the stroke patient with some residual effects of stroke at home, as the patient was reported to have fully recovered after the discharge. The family caregivers whose stroke family member died soon after discharge were deemed as having insufficient experience in the care of stroke patients.

The inclusion criteria for stroke patients included having been admitted to the hospital due to stroke as reported and confirmed by the families or patients themselves, having acceptable verbal communication capacity, and being discharged to their own home with some degree of disability or problems in activities of daily living. In general, the process of recruiting patients and families were carried out concurrently. 
All the potential participants were approached via telephone, where a brief explanation about the study, its purpose and the procedures for data collection was given. After the explanation, people were invited to participate in the study. Once verbal agreement was obtained, the date and venue for the interview were fixed as suggested by participants. The initial plan was to pay a social home visit before the interview session so as to establish rapport with the family and study participant. However, all the participants preferred for the interview to be held during the first meeting. The details of the study as contained in the information sheet were explained before obtaining written consent from study participants. Further details on the procedure of the data collection are explained in the section on data collection method.

\section{Recruitment strategy for health professionals}

For practical reasons the process of recruitment varied slightly for each category of healthcare professionals. The health professionals were chosen from persons who were involved in stroke care, at senior level or knowledgeable about the issues in the study. They included therapists from the rehabilitation unit, medical doctors who were in charge of providing the service in stroke care, academic nursing staff, registered nurses and nurse managers. All potential participants were personally approached by me to participate. The head of department and other staff did not know who was involved and who agreed to be a study participant. The rest of the health professional participants were identified by me and personally approached to participate in the study. Each participant was given a copy of information sheet. The details of the study were explained before obtaining written consent from study participants.

\section{Recruitment strategy for a key member of NASAM}

NASAM was established in 1996 as a non-profit organisation providing services for stroke survivors in Malaysia. It initially began as a stroke support group formed by a former stroke survivor who had a remarkable recovery from stroke. The overwhelming support and response received from the public inspired the NASAM's Founder Chairman to establish the first NASAM centre at Petaling Jaya, Selangor in 1996. Selangor is one of the states in the West Coast of Peninsular Malaysia. Since, then, many other centres 
have opened in other states of Malaysia such as Perak, Melaka, Sabah and Johor, but no centre in Kelantan. NASAM's two main objectives are to provide rehabilitation service to enable stroke survivors to return to as normal a life as possible within the limits of their disabilities and to promote the concept of stroke prevention by raising public awareness on the risk of stroke.

The initial contact with NASAM was made via e-mail before I went to Malaysia for data collection. I was referred to a person at NASAM who was deemed by the person in charge as most appropriate to be a study participant. After correspondence via several emails, s/he was invited to be a study participant as s/he fulfilled the inclusion criteria. This was based on their position and experience in stroke care at NASAM. A set of information related to the study such as information sheet, consent form, letter and flyer requesting volunteer to be interviewed and introductory letter from supervisor (Appendix 7, $8 \& 9$ ) were given to the potential participant after which they agreed to be a study participant. It was agreed that the interview session be conducted sometime in early March 2008.

\section{Data collection methods}

Obtaining information and insights from the study participants was one of the most important activities in the research study. Such information and insights would be dependent on the design of the research method, data collection procedure and the research tools. As the core activities of the data collection in the Phase 1 of the study was to elicit information from various group of participants, it was decided that personal interviews using the semi-structured interview guide was the best method to explore the resource needs of home-based stroke patients and their family caregivers.

\section{Interviews}

As Hutchinson and Wilson (1992) indicate interview questions must fulfil the research purpose to avoid threats to validity. Therefore careful consideration was given to developing a valid interview schedule. In-depth interviewing was chosen as a method of data collection to obtain rich information about the focus of the study. The semi- 
structured interview is the most common method of data collection in qualitative research compared with unstructured or structured interviews (Burnard, 2005). In-depth interviewing is defined as 'repeated face-to-face encounters between the researcher and informants directed toward understanding informant's perspectives on their lives, experiences or situations as expressed in their words' (Taylor \& Bogdan cited in Minichiello et al., 1991).

Patients and caregivers, a key person from NASAM and other health professionals were interviewed to gain an understanding of the current practices of health professionals in relation to education for patients and families during the acute stage, prior to discharge and while at home. It was also to gain an insight into the needs of stroke patients and their caregivers during early rehabilitation and the area of information and education needed.

Key demographic data and characteristics of the patients and caregivers such as age, gender, types of job, education level and marital status were gathered. Three different sets of interview guides were used for the stroke patients and family caregivers, health professionals and the key person from stroke association respectively. The interview guide for the patients and caregivers covered five main areas: (i) life changes after stroke and management at home, (ii) the current practice of health professional in relation to provision of education and health information, (iii) interaction with other people and community, (iv) information needs, and (v) other needs for effective rehabilitation in the home.

The interview guide for the health professionals focused on eliciting information and opinions on four main issues: (i) the important aspects of early rehabilitation at home, (ii) the current practice related to acute stroke care and the provision of information and education, (iii) information needed or that should be included in the educational module, and (iv) the interfaces between health service and stroke patients and their caregivers post-discharge. With respect to the interview guide for the NASAM member, four main issues were explored: (i) NASAM's activities and contributions in relation to the health 
needs of patient post-stroke, (ii) the important aspects for early stroke rehabilitation, (iii) NASAM's strategies to strengthen the relationship between NASAM and home-based stroke patients and family caregivers, and (iv) educational needs.

\section{Pilot interviews}

As recommended by Birks, Chapman and Francis (2007) two pilot interviews were conducted before the main interviews. Pilot interviews were carried out with a family caregiver and a health professional who were not from the group that were involved in the study. Both of these people were considered as representing the group of all caregivers and health professionals. The data from the pilot interviews were not analysed as the purpose of these interviews was to gain familiarity with the recording instrument and the interview guides as well as to identify obstacles that might arise during the interviews. It was also to familiarise myself with the interview situation in Malaysia, as the interview schedule was developed while I was in New Zealand.

The first thing that I learnt from the pilot interviews was how to bring the participants back to the issues or the focus of the study. The family caregivers had a lot of experience in caring for their stroke family members and tended to reveal a wide range of information from their caregiving role experience, about other family members, personal issues and other workloads. It was decided that the recording instrument would not be put on "pause" or switched off anytime until the interview was finished. Any unexpected interruptions during the interview could be excluded during transcription.

\section{Interviews with stroke patients and family caregivers}

Five discharged stroke patients and five family caregivers were chosen for these interviews. My position as a former registered nurse at HUSM and as a nursing lecturer gave me some advantage in putting the interviewees at ease during the telephone conversations and interview sessions and subsequently obtaining their trust. From my experience during the pilot interview, I found that I needed to create a relaxed and less formal interview in order to get their co-operation. I consistently showed my interest in the issues expressed by the participants. All of the participants agreed that the interviews 
could be audiotaped. Having realised the possibilities that the questions might not be fully understood by the participants, certain precautions were taken as part of the interview. The interviews were conducted using the Kelantanese dialect on top of the standard Malay language. The type of language used was based on the patients' and families' background such as age, education level and socio-economic status. For example, communication with patients mostly used the Kelantanese dialect. As I am well-versed in both the Kelantanese dialect and standard Malay language and the study participants were all Kelantanese and spoke the Malay language, there was no issue relating to language and communication. Questions were addressed in a normal conversation manner in a straight forward, clear and non-threatening way and participants were given ample time to speak freely and openly. It was emphasised to the participants before the interview sessions that their honest and frank opinions were welcome.

All the interview sessions with the patients took place in their homes as requested by participants. They were all done in the presence of their family member or other people who were close to them. This may be mainly due to their health conditions which require them to be accompanied by another person. In contrast, interviews with the caregivers were conducted at various places such as the workplace, their own home and at my house which ever was considered more convenient to them. From the beginning, I consistently emphasised to the participants that the selection of time and venue for the interview sessions was solely at their discretion and convenience. This was because I could accommodate any time or place selected as I was on full-time study leave.

\section{Interviews with health professionals}

To reiterate there were 12 health professionals from various disciplines involved in the interviews. The same interview guide developed for health professionals was used for 11 out of 12 participants, and a separate interview guide was used for the representative from NASAM. There were some differences in terms of the language used between the patient and caregiver participants compared with health professionals. For the interviews with the health professionals, there was more usage of the standard Malay language, 
mixed with the English language or medical terms compared with those of the stroke patient and caregiver participants. With regard to the interview data, all the health professionals were more interested in the findings of the study than checking the interview transcripts. Therefore it was decided that interview transcripts would not be returned to the health professionals until the findings of the Phase I were presented during the Phase 2.

Twenty-one of the 22 interviews were conducted in the Malay language with local dialect to ensure optimal understanding. One interview was conducted in English as requested by the participant because of their fluency in English rather than Malay. It is accepted that interviews should be conducted in a familiar language that can be understood by both the interviewer and the interviewee (Kvale, 1996). The interviews took place at a time and place suitable and convenient for both the interviewer and interviewee and to avoid any external interruptions. The costs of reasonable expenses to attend the interviews were covered by the researcher as requested by participants. The interview duration ranged from 20 minutes to 110 minutes.

\section{Analysis of the interview data}

The analysis of the interview data was done manually. In this study, interview data were transcribed manually by jotting down every word of conversation of the participant involved which is known as "verbatim transcription" (Minichiello et al., 2008). Data from both phases of this study were analysed using thematic and content analysis. According to Braun and Clarke (2006) "thematic analysis is a method for identifying, analysing and reporting patterns (themes) within data (p. 79)". In general it is a method of analysing data for themes or patterns. This type of analysis involves the search and identification of common threads from an entire interviews or set of interviews (Morse \& Field, 1996). They further describe that frequently, themes are concepts identified from the data, rather than concrete entities expressed by the participants. The theme appears obvious once identified. Interview transcript from my data was then read and reread by me to search for themes. The themes that emerge were indentified based on the research questions and the objectives of the study. As stated by Braun and Clarke "A theme 
captures something important about the data in relation to the research question, and represent some level of patterned response or meaning within the data set” (p. 82).

A number of evidences support the use of thematic analysis as a useful method for analysing data in qualitative research (Attride-Stirling, 2001; Braun \& Clarke 2006). With regard to my study this method of analysis assist me in identifying issues related to educational needs of patients and families. More specifically, thematic analysis was used because

the focus is to identify themes emerging from the transcripts recorded through interview with the informants. The process by which this is achieved is by the researcher engaging in a systematic examination and re-examination of the data line by line in order to obtain meaningful categories that can be grouped to form a theme or a series of themes. (Minichiello et al., 2008, p. 280)

In the context of the study, a systematic examination and re-examination of the data lineby-line was based on the Malay text. This is because data from the interviews were initially transcribed verbatim in the Malay language. Verbatim transcription was carried out to ensure that the real meaning of the conversations was maintained. Although transcribing word-by-word is time consuming, in my experience with the transcribing process I found that verbatim transcription was useful in ensuring the accuracy of the meaning of the conversation. In certain cases, omission of one spoken word may give rise to a different meaning altogether. I also found that verbatim transcription saved time in the later stages of analysis. Lapadat and Lindsay (1999) indicate that verbatim transcription is a valuable process because understanding and analysis takes place during "listening and re-listening, viewing and re-viewing" (p. 82) and also facilitates interpretive thinking and close attention in order to make sense of the data. All the transcriptions of the interviews were done by me. Some researchers suggest that transcribing could be done by professionals with skills in transcription. However, Tilley (2003) argued that researchers should do the transcribing themselves to avoid missing "out on the kinds of understandings that develop during transcribing process as well as lose control over some of the transcription decisions made when they do not do the work 
themselves" (p. 770). Dearnley (2005) pointed out another issue, that if a professional service was used for transcription great care should be taken to maintain participants' confidentiality.

The transcribing process was assisted by use of good standard speakers which helped to produce fine acoustic quality. The completed transcripts were re-read and compared several times with the tapes to ensure no word was left out from the transcript. The transcription from each participant was recorded on a separate written sheet and coded (based on the categories of stroke patient, caregivers or health professionals).

The interview texts were placed in a table with two columns for the purpose of the English summary. The first column contained an English summary and the second column was the verbatim transcription in the Malay language derived from the tape. By doing the English summary, familiarisation with the data increased. This was enhanced because I was involved with the whole process of interviewing and transcribing from the very beginning. Familiarisation with the data is one of the steps in qualitative content analysis. Familiarisation is achieved by "listening to tapes, reading transcripts, studying notes and so on, in order to list key ideas and recurrent themes" (Pope et al., 2000, p. 116). In addition, part of the content analysis activities was carried out as the interview texts were read many times for the purpose of doing the English summary. Tapes were re-played when necessary to reconfirm the exact meaning of the interview text. Kvale (1996) contended that analysis also takes place during repeated listening of the tapes and cutting and pasting of the interview text.

Before doing the analysis, a few steps were taken to assist in the analysis process as well as to maintain participants' confidentiality. These included storing the original transcripts safely in different places: in the computer, thumb drive and compact disc. Three sets of printed copy were made; two sets were kept in a safe place and the other set was used for doing data analysis. The whole content of the final transcripts of the study participants were checked. The names of the participants who sometimes appeared in the interview transcript were removed so that the transcript could not be identified to a 
particular participant. The three groups of study participants were first analysed and presented separately.

The analysis of the participants' interviews was based on the interview transcript and notes from the informal conversation and observations to aid my understanding. The entire interview transcript was first read a few times to get the general idea or impressions of the whole interview which were then recorded. Then, the interview transcript of each stroke patient was read several times to get the general ideas or to identify the key issues relevant to the data. In certain cases, I replayed the tapes to listen to the interview or referred to the notes to confirm the real meaning from the interview text. These activities were done so that I would better grasp the data and enhance my appreciation of it. The whole analysis was based on the Malay transcript. The units of analysis were sentences or phrases. Then the general ideas or issues arising in each transcript were grouped and listed. The themes from the list of general ideas and concurrently from each transcript based on the research objectives are searched. The keywords or meaning unit that may lead to a theme in each transcript were marked or underlined using different colour markers. Sandelowski (1995b) state, getting a sense of each interview is required before cross comparisons between the data. Following that, themes from the interviews with each group of participants were identified based on the objectives of the study. Thematic analysis in each transcript was carried out and comparison of the analysis made between the transcripts. All the final themes that emerged from the analysis of each group of study participants are presented in Chapters 6 and 7.

Further, integrated analysis was done from the findings of the three groups of study participants. This analysis involved the triangulation and integration of the findings from each group of participants and is presented in Chapter 8.

\section{Ethical consideration in Phase 1}

Ethical issues are core to all steps in the research process. Informed consent was obtained from all the participants prior to conducting the interview. An example of the 
informed consent is shown in Appendix 7. Participation in the study was voluntary. No one was coerced to participate. The principles and procedures for obtaining informed consent were strictly followed. The study purposes and the methods of data collection were explained in detail to the participants. It was also emphasised to the participants that they had the right to withdraw from the study at anytime they wished and this was reminded to them throughout the data collection process. In addition, the participants were informed that the information or data provided would be kept confidential and would be utilised solely for the purpose of the study. Confidentiality was maintained throughout the study.

\section{Phase 2 of the study - design and process}

The direction of the second phase of data collection emanated from the completed analysis of Phase 1 data involving health professionals', family caregivers' and stroke patients' interview data. Phase 2 took place approximately one year after Phase 1 data collection. An extension to the ethics approval used in Phase 1 was obtained. This extension included the development of additional information sheets, consent and interview guide (Appendix 10 and 11). From the first phase it was evident that health professionals were very clear that healthcare professionals needed to be involved in developing educational strategies for home-based stroke patients and their family caregivers.

This second phase involved working with health professionals who were interviewed in Phase 1. To reiterate, for the purpose of this study, the various health professionals involved were considered to be expert in their own working area related to post-stroke care. The main aims of this second phase were to discuss what was raised in the interviews from Phase 1 about health education needs of home-based stroke patients and their family caregivers and to identify priorities and processes for how these needs might be addressed. Further aims were to establish what information/resources were presently available that people can access and to create strategies for going forward based on the findings. In this second phase, it was pre-determined that only 11 health professionals would be involved compared with 12 in Phase 1. One of the health professionals was 
excluded due to the inability to join focus group discussions conducted in the Malay language. Data were collected using focus groups and individual interviews. Having participants from the same workplace provided an advantage for me to approach them personally. Therefore the 11 health professional participants from Phase 1 were approached and invited to participate in Phase 2. Potential participants were given the information sheet and detailed information about the purpose of the focus group and how it would be held. My role as facilitator or moderator was emphasised to them. All of the participants who were approached were very interested to share and contribute their ideas for the development of education strategies for HBSP and family caregivers.

The intention was to conduct two focus groups each comprising five and six participants. However, due to the inability to set a time to accommodate all group members, two participants declined to participate. A third participant was also unable to participate due to personal reasons. On the day of the focus groups two participants were unable to attend due to unanticipated reasons. These participants agreed to be interviewed individually. Therefore of 11 participants, 8 participated in the second phase of the study. Six participants participated in focus groups with three participants in each group and two were interviewed. Some researchers suggest that the composition of focus group should range from four to seven. However, other researchers have argued that although small, three is still an appropriate number of participants for a focus group (Kitzinger \& Barbour cited in Barbour \& Kitzinger, 2001). Also a small number in the group is acceptable "when participants have expertise on the topic" (Kruger, 1995, p. 2). I agree, however, with Smith (1995) who indicates that group cohesion is easy to maintain in a smaller group.

In preparation to run the focus group, I conducted a practice session with five nurse educators to ensure I was a skilled facilitator and was confident in conducting a focus group with health professionals. The other purpose of this practice exercise was to familiarise myself with the focus group guide as well as the effectiveness of the recording instrument. The type of recording instrument used was found very effective and was able to captures all the voices from various angles. 


\section{Focus group and interviews}

Coolican (2004, p. 165) defines a focus group as a "group with common interest who meet to discuss an issue in a collective interview in order for researchers to assess opinion". Focus groups were considered important to complement the individual interviews because "group processes can help people to explore and clarify their views in ways that would be less easily accessible in a one-to-one interview" (Kitzinger, 1995, p. 299). In addition, focus groups are able to provide rich and detailed perspectives through group interactions which could not be obtained using other methodological strategies (Krueger, 1994; Lam et al., 2001; Morgan, 1993). The inevitably distinct difference with the one-to-one interview or questionnaires is the interaction between participants (Kitzinger, 1994).

Although there is an increasing interest in the focus group as a method for nursing research (Kitzinger, 1995) and in the study of health, health care and other social processes (Sim, 1998), very few research articles using this approach in Malaysia were found, especially focus groups among the health professionals. Ali (2001) in her PhD study conducted focus groups with antenatal mothers and midwives in a rural district in Malaysia to explore their views related to antenatal care practice. Focus groups were also conducted among Malaysian medical and nursing students to examine their views related to teaching strategies (Lam, Irwin, Chow \& Chan, 2001). In this second phase, the focus groups were conducted as the main data collection method to get consensus on the priorities and processes for developing educational strategies. To reiterate, these focus groups consisted of the key members in stroke care who were interviewed in Phase 1. It is congruent with Grbich (1999) who indicates that an ideal focus group is a group of key informants who come together to discuss issues on a particular topic. The focus groups were able to encourage discussions and debates among the participants in prioritising the educational resource needs and allow me explore participants' opinions and ideas. As Kitzinger (1993) demonstrated, interactions between participants can highlight their understanding and priorities as well as encourage an open conversation and permit the expression of criticism. Furthermore, focus groups promoted exchange of 
views and ideas as well as insights which are unlikely to surface during individual interviews.

As the health professional participants were among the key person in stroke care with many years working experience, this focus group may represent the general population of health professionals within the study settings. Careful consideration was given to ensure the homogeneity of participants in the groups. For example, participants were placed in a group with almost similar working experience to promote open discussion and interaction between participants. Homogeneity was encouraged within the group to capitalise on people's similar experiences (Kitzinger, 1995).

The majority of the participants preferred that the focus group be conducted during working hours. However, due to some constraints related to work-related issues, the first focus group was held during the weekend. The second focus group was conducted during working hours. The venue and the time for the focus groups were agreed by all parties. Careful consideration was given to ensuring the setting was convenient for free conversation and for the purpose of audio-taping the conversation, such as being fully air conditioned, quiet and free from any distractions. Maintaining a favourable environment is important to ensure free and open discussion between participants in the group (Kitzinger, 1995) as well as enhance quality of data (Krueger, 1995).

The focus group commenced with an informal conversation. As all the participants in this second phase were known to me as they came from the same workplace and through involvement in the first phase of study, there was no issue in establishing rapport with them. Participants also had a working relationship with each other. This was an advantage that provided comfort and stimulated discussion between participants in the group. Another contributing factor towards free and open discussions was the homogeneity among the group members in terms of coming from the same workplace. Furthermore, with respect to the hierarchy of the job status, there were not many differences amongst the participants. 
All the participants needed to sign a consent form before the focus group commenced. I started the session by revisiting the purpose of the focus group and reiterated the ground rules as stated in the information sheet. Participants were reminded of the need to keep the contents of the group discussion confidential. Then, the findings from Phase 1 relating to educational needs of HBSP and their family caregivers were presented as a basis to commence the group discussion. The presentation was in topics. The Phase 2 of this study was conducted using a semi-structured interview guide (Appendix 11). The interview guides were developed to explore participants' views on the priorities and processes to develop an implementation plan for education strategies for HBSP and their family caregivers at home.

\section{Researcher as moderator and facilitator}

The role of moderator or group facilitator is very important in a focus group discussion (Fern, 2001; Litosseliti, 2005; Sim, 1998). The focus group moderator should have specific characteristics for successful focus group discussion such as good personal, interpersonal, communication and managing skills, be 'neutral', opinion-free, nonjudgmental, confident and in control, flexible and adaptable (Litosseliti, 2005). Fern argued that the moderator may need different skills and personalities with different focus group tasks. She also suggests that the moderator should come from the same population as the focus group participants. With regard to this issue, I as moderator for the focus group was also from the same population group as the participants. As the task of moderator is similar to being the interviewer in face-to-face interviews (Berg, 2007), Berg suggests that the moderator should have a guide which I applied in this study.

Some researchers have discussed the characteristics of a good focus group moderator as having professional qualification and training skills. However, Fern (2001) argued that not all focus group moderators need to be highly skilled professionals because "no single set of moderator characteristics is adequate for all focus groups; different research purposes requires different moderator" (p. 73). This is supported by the earlier views of Morgan and Krueger (1993) who indicate that the focus group moderator does not necessarily need to have professional moderation skills. I agree with Kitzinger and 
Barbour (cited in Barbour \& Kitzinger, 2001) who suggest that a simple way to develop moderator skills is by conducting focus group session with colleagues, friends, students or relatives, which I applied in this study by conducting practice sessions with colleagues.

As a facilitator, I always tried to keep myself unobtrusive and facilitative rather than intrusive and directive (Nyamathi \& Shuler, 1990). However, as Curtis and Redmond (2007) suggest, a focus group is not a passive function and facilitators should encourage discussion within the group by asking questions and elicit responses. Litosseliti (2005) specified the three main roles of moderator during a focus group session are to maintain the group's focus and keep the discussion on track, guide, stimulate and facilitate the discussion and put participants at ease. To avoid distraction and to focus the attention on the discussion, notes were not taken during the discussions. As soon as the session finished, I wrote notes of what I recalled were the key issues or important points raised. These were to complement what was recorded. The process of conducting the interviews was similar to the individual interviews in Phase 1. The guide for the focus group was used for the individual interviews.

\section{Analysis of Phase 2 data}

Similar to the experience in conducting individual interviews for the first phase of study, data from Phase 2 were transcribed on the day of the group session. There were some differences in term of transcribing the focus groups and the interviews from the first phase of study. It was intended before the process of data collection that focus group and individual interview data would not be transcribed verbatim, rather the focus of transcription was to extract the key topics and ideas that emerged. Conversations held with individuals before and after the focus groups were also used in the analysis. In general, however, the process of analysing data for Phase 2 was similar to the first phase of this study.

The processes of analysing focus group data are similar to analysing other qualitative data (Kitzinger \& Barbour, in Barbour \& Kitzinger, 2001). However, care was taken to ensure that data were analysed "of the context of group interaction, not individual 
comments" (Asbury, 1995, p. 417). This is supported by Morgan (1995) who stated that the unit of analysis is the group, not the individual. He also added that any individual voice in the group is influenced by the group dynamic.

\section{Ethical issues in Phase 2 (during focus groups and individual interviews)}

Some of the ethical issues were similar to those of the first phase of the study. These include obtaining informed consent, maintaining confidentiality and participants' rights. Informed consent (Appendix 10) was obtained from all the study participants prior to conducting the focus groups and individual interviews although the same participants had been involved in Phase 1. The same principles and procedures for obtaining informed consent were strictly followed. Participation in the study was voluntary. No one was coerced to participate. The aims of this Phase 2 data collection through focus group and individual interview were explained in detail to the study participants. It was also emphasised to the participants that they had the right to withdraw from the study at anytime they wished but in focus groups could not withdraw their data. In addition, the participants were informed that the information or data provided would be kept confidential and would be utilised solely for the purpose of the study. With respect to the focus group, confidentiality was again given attention. Participants were also encouraged not to disclose any information outside the group.

\section{Considerations of rigour}

Mays and Pope (1995) contended that "the basic strategy to ensure rigour in qualitative research is systematic and self conscious research design, data collection, interpretation, and communication" (p. 110). As already indicated, the interview schedule was carefully developed to ensure it fulfilled the research objectives to avoid validity threat. The recruitment of participants using purposive sampling and snowball sampling methods enabled me to obtain rich data as participants were chosen from those with relevant and first-hand experiences, as well as being experts related to the focus of the study. 
In order to ensure the validity of the data collected, a few issues were considered prior to data collection. Hutchinson and Wilson (1992) indicate that timing of interviews is critical to obtaining valid interview data. In regard to this, participants were given the choice to decide on the interview time and venues and a variety of places for interview were chosen based on participant's convenience. I was always prepared for any interruption that might happen during the interview. For example, of those 22 interviews conducted, one of the interview sessions with caregivers was stopped and cancelled after five minutes and was continued the next day due to the emotional impact on the participant. I realised that the interview session should be conducted in another place without the presence of their stroke family member. Hutchinson and Wilson claim that good interviewers should apply some flexibility and be able to conduct quality interviews in a variety of places.

In addition, sufficient time was provided for establishing rapport, clarifying certain issues when needed and conducting the interview. Every effort was taken to ensure that interviews were conducted in a conducive, interactive environment and non-threatening manner. Openness to what people said was maintained throughout the data collection process. Everyone's voice was treated as being important and respected. While in the focus group, I chose a homogeneous group so that it would stimulate interaction between group members and allowed everyone the chance to have a voice. Mays and Pope (2000) pointed out that triangulation could be in the form of comparing the result with different data collection method. In this study, the triangulation of the findings of the various groups of participants added to the rigour of the study.

A high quality tape recorder was used to prevent any error or malfunction during the data collection. The recording instrument was checked prior to each interview in terms of sufficiency of power and function to prevent technical failure. If any interruption occurred during the interview session, I did not switch off the tape or turn to "pause" to prevent discontinuity or any unexpected malfunction during the recording session. Instead, any interruptions captured by the recorder were later excluded from the transcription. All 22 interviews sessions were conducted by me which included audio 
taping the interview and completing the transcription. This was to ensure that the credibility of the data collected was sustained.

During the interviews, I always made myself aware of my role as a researcher and not as an educator. This is to ensure that there is no changing role between the interviewer and the interviewee. This is particularly important in the situation where the focus of the interview is to identify the educational needs of patients and families. Some patient and family participants may require some information or indicate some misunderstanding on certain concepts. According to Hutchinson and Wilson (1992) the quality of the research data suffers due to the changed role of the interviewer. However, they were agreed that "sharing information or honest reassurance is not totally inappropriate, but the predominant role of the interviewer should not be that of educator, therapist, or caretaker" (p. 118).

All the interviews were conducted in the participants' own language. In relation to this, Twinn (1998) claimed that the importance of interviewing in the first language of participants is to maximise the quality of the data obtained. Transcription is central to the process of analysis (McLean et al., 2004). Furthermore the analysis process was conducted based on the Malay language used during the interview. Twinn suggested that where possible the analysis of the transcripts should be conducted in the first language of the interview. Translation from Malay to English was done with careful consideration to retain the real meaning of the conversation. Therefore there is no issue of losing the data during the translation. In addition, conducting the English summary increased familiarisation with the data. In regard to validity in the focus group, Nyamathi and Shuler point out that "typically focus groups have high face validity, due to the credibility of comments from participants" (1990, p 1284). This is clearly reflected in this study as all the health professional participants were the experts in relation to the issues of the study. As described in data analysis section, data analysis was conducted in a systematic way to ensure all the themes emerged in the data were covered. RyanNicholls and Will (2009) indicated data that are collected and analysed systematically and findings presented correctly are part of the rigour of the research. 
Researchers have suggested that face validity or member checks or respondent validation are techniques to improve rigour and increase credibility in research (Appleton, 1995; Girbch, 1999; Mays \& Pope, 1995; Miles \& Huberman, 1984 cited in Sandelowski, 1986). I applied in this in this study by presenting back the findings from Phase 1 to the same participants in Phase 2 through focus group and individual interview.

\section{Conclusion}

This chapter has described in detail matters relating to the procedures of Phase 1 and Phase 2 of the study which were conducted in Malaysia and HUSM as the study site. Stroke patients and the family caregiver participants were selected from those who were discharged from this hospital. Moreover, a few health professionals were also selected as professional participants. The data in Phase 1 of the study were collected via in-depth interview using a semi-structured interview guide and were supplemented by the field notes. The data for Phase 2 of study were collected using focus groups and individual interviews comprising the health professionals who had been involved in Phase 1 . Verbatim transcription was used for the individual interview data in the Phase 1 of study. Data from both phases of study were analysed using thematic and content analysis. As mentioned, the validity and reliability as well as the ethical issues were given attention throughout the study. Chapters 6,7 and 8 will present the findings from Phase 1 and Chapter 9 the findings from Phase 2 of the study. 


\section{Chapter 6: Process and findings of the analysis of stroke patients and family caregivers}

\section{Introduction}

This chapter presents the findings of the interviews with the first two groups of participants - stroke patients and family caregivers. The findings of the interviews with health professionals are presented in Chapter 7. Data gathering for Phase 1 for all the three groups of participants was undertaken over the two month period of February and March 2008. Data were obtained using semi-structured interviews with five stroke patients, five family caregivers and 12 health professionals. Each interview was tailored to who the participant was. Except for one couple from the same household, all patient and caregiver participants were not from the same household and were unrelated. Each person was interviewed by me to gather information on what resources are needed by stroke patients and their family caregivers. All the interviews were conducted during the day and were preceded by a general conversation.

The chapter commences with the general background of the patients and caregivers. It is followed by the stroke characteristics of the interviewed patients. The findings are the presented in two parts: those of patients and those of caregivers. The details of the findings are described under the thematic headings.

\section{Patient participants}

Face-to-face interviews were conducted with the stroke patients who had been discharged from the hospital and lived in their own home with family caregivers. The focus of the interviews was mainly on the life changes and home management after stroke and the health education provided by health professionals during the acute stage and post discharge. The interviews covered the education and information received and what was needed by stroke patients.

The detailed procedure for recruiting patients was explained in the methodology chapter. A total of 21 stroke patients were considered for eligibility to be a study participant but 
16 were excluded from the study. The reasons for exclusions varied. The majority $(\mathrm{n}=8)$ were unable to verbally communicate, four had died soon after discharged, two declined to participate and two were unable to be contacted on the phone number provided.

\section{Findings from stroke patient interviews}

The patients were all interviewed in their home as requested by participants and agreed by the interviewer. The interviews ranged in time from 17 minutes to 42 minutes, with an average of 29 minutes. All patients could speak and had normal understanding of the conversation. Four of the participants were Malays and one was Chinese. All interviews were conducted in the Malay language. In four of the interviews at least one family member was present and contributed to parts of the interview. In the fifth interview, the patient was accompanied by her neighbour because family members were at work. The majority of the patient participants were from the lower to moderate education level and socio-economic background as none of them had obtained tertiary education. Two of the five had no formal education. All patients lived with some family members, the wife or husband and their children. Table 2 summarises the participants' socio demographic characteristics.

Table 2. Socio-demographic characteristics of interviewed stroke patients

\begin{tabular}{|l|l|l|l|l|l|l|}
\hline $\begin{array}{c}\text { Parti- } \\
\text { cipant }\end{array}$ & Gender & Age & Ethnicity & \multicolumn{1}{|c|}{$\begin{array}{c}\text { Education } \\
\text { level }\end{array}$} & $\begin{array}{l}\text { Occupation } \\
\text { before stroke }\end{array}$ & Marital status \\
\hline SP 1 & Male & 64 & Malay & $\begin{array}{l}\text { Completed } \\
\text { primary } \\
\text { school }\end{array}$ & $\begin{array}{l}\text { Owned and } \\
\text { worked in a } \\
\text { mirror shop }\end{array}$ & $\begin{array}{l}\text { Married with seven } \\
\text { children }\end{array}$ \\
\hline SP 2 & Male & 43 & Malay & $\begin{array}{l}\text { Completed } \\
\text { secondary } \\
\text { school }\end{array}$ & $\begin{array}{l}\text { Assistant } \\
\text { surveyor }\end{array}$ & $\begin{array}{l}\text { Married with four } \\
\text { children }\end{array}$ \\
\hline SP 3 & Male & 58 & Malay & $\begin{array}{l}\text { Never been to } \\
\text { school }\end{array}$ & Builder & $\begin{array}{l}\text { Married with five } \\
\text { children }\end{array}$ \\
\hline SP 4 & Female & 40 & Malay & $\begin{array}{l}\text { Completed } \\
\text { primary } \\
\text { school }\end{array}$ & Housewife & $\begin{array}{l}\text { Married with five } \\
\text { children }\end{array}$ \\
\hline SP 5 & Female & 63 & Chinese & $\begin{array}{l}\text { Never been to } \\
\text { school }\end{array}$ & Housewife & $\begin{array}{l}\text { Married with three } \\
\text { children }\end{array}$ \\
\hline
\end{tabular}


Based on the interview content and researcher observation, all interviewed patients still had some effects of stroke during the time of the interview session such as numbness or weakness of the upper or lower limbs. The status of functional disabilities after the onset of stroke was based on patients' and caregivers' description. Table 3 summarises the stroke features at the time of the onset of stroke of those interviewed.

Table 3. Stroke characteristics at the onset of stroke

\begin{tabular}{|l|l|l|l|}
\hline Participants & $\begin{array}{c}\text { Duration of } \\
\text { hospitalisation }\end{array}$ & $\begin{array}{l}\text { Functional disabilities after the } \\
\text { onset of stroke }\end{array}$ & \multicolumn{1}{|c|}{$\begin{array}{c}\text { Other co- } \\
\text { morbidities }\end{array}$} \\
\hline SP 1 & 4 days & $\begin{array}{l}\text { Aphasia, right side paralysis, } \\
\text { unable to walk }\end{array}$ & None \\
\hline SP 2 & 3 days & $\begin{array}{l}\text { Aphasia, right side paralysis, } \\
\text { unable to walk }\end{array}$ & None \\
\hline SP 3 & 2 days & $\begin{array}{l}\text { Numbness of the left side, unable } \\
\text { to walk, }\end{array}$ & Hypertension \\
\hline SP 4 & 2 weeks & $\begin{array}{l}\text { Right side paralysis, unable to } \\
\text { walk. }\end{array}$ & Hypertension \\
\hline SP 5 & 4 days & $\begin{array}{l}\text { Left side paralysis, unable to } \\
\text { walk }\end{array}$ & $\begin{array}{l}\text { Hypertension and } \\
\text { diabetes }\end{array}$ \\
\hline
\end{tabular}

The patient interviews were based on the objectives of the study:

1. to identify the area of education or information needed by home-based stroke patients and family caregivers based on their perceptions and those of healthcare professionals.

2. to identify the current practices of healthcare professionals in relation to information or education needed by home-based stroke patients.

3. to identify the important aspects of early rehabilitation at home.

The findings are presented under thematic headings. Supplementary information was obtained from the field notes or informal discussion before or after the interview and from observations. Quotations from patients have been chosen on the grounds of representativeness. Sometimes I need to add my words in the excerpts to aid understanding. Whenever I have done this, the words are put in brackets [ ]. 


\section{The themes}

In general, eight major topics emerged from the stroke patient interviews,

1. Information or education needs: for fast recovery, good medication, home management, causes and how stroke occurs and managing co-morbidity conditions.

2. Urutan tradisional and exercise as an important aspect of early stroke rehabilitation.

3. The impact of stroke: on social life, behaviour and emotion of the patient, and work changes after stroke.

4. Coping strategies or dealing with the impact of stroke: spiritual and religious help, avoiding stress and promoting socialisation.

5. Services and information or education received.

6. Support needs: support needed at home, and from the health service.

7. The benefit of meeting other stroke patients: reducing stress and feeling better, sharing experience on a strategy for recovery.

8. Hoping for the future: able to manage self and be independent.

Each of these main themes are presented separately.

\section{Information or education needs following a stroke}

Most patients interviewed did not openly voice concern about the lack of information they received at the time or following the stroke. Interviews revealed there was a lack of information on many aspects related to stroke care. All patients wanted information and education on a range of topics how these were differences in what each person wanted. Information needs involved how to have a faster recovery, the best medications to use, what causes stroke and how to manage at home.

\section{(i) Information need related to stroke and stroke care}

One of the participants (SP 2) stressed the need for information about how to recover faster and the best medication for stroke. 
I do not know. The doctor should tell how to recover faster ... should tell how to recover faster and recommend the best medication. Nowadays, I still can feel the numbness.

Similarly, participant SP 2 was keen to know about faster recovery. "I want to know how to recover faster, but the doctor just tells that the blood is okay, nothing else". Participant SP 1 also experienced a lack of information about speeding up recovery.

The doctor did not tell anything. I just go once a month to check the blood, the result is the same. Check again and the result is still the same. No difference.

This participant commented that he considered the needs of stroke patients varied, depending on the individual's circumstances, and because the effects of stroke on individuals are different.

Stroke is different, this person was affected like this and the other person was affected in different way, it is not the same. They are different. It is hard to explain. How to say ... because of the effects of stroke are different. They are different. Some had affected the brain, some had affected the hand.

It was evident that education and information need to be tailored to individual needs. For example, the needs of younger stroke patients were different from those of the older patients. Participant SP 2 as a younger stroke patient was really keen to learn to drive again. His need was to find out whether the hospital rehabilitation unit provides rehabilitation service related to driving. In his view he would be able to drive with some support and training from rehabilitation staff.

Patients also expressed a need for information on "how stroke occurs". For participant SP 2 the need was to understand how stroke still happens even though there are "no signs" of it. Participant SP 4 stated that she did not receive information or education related to stroke itself or management at home. However, a doctor had told her to control her hypertension by eating a low salt diet. Understanding the cause was related to prevention. Participants wanted information about whether they could have prevented a stroke, and how they could prevent one occurring in the future. 
Some patients also talked about their experience in relation to care giving. They wished that their caregiver had been given information and education on how to care for them at home. Participant (SP 2) said that

If possible, hospital staff should tell and teach the caregivers how to care for the patient at home, especially during the early stage because of no experience.

(ii) Information needs on managing co-morbidity conditions

When asked how health professionals gave information on the prevention of stroke recurrence or the management of other conditions such as hypertension participants reported that the information given was limited and lacked explanation. As SP 3 stated "He [doctor] said ... less fat ... salted fish ... sugar ... that's all. Nothing ... he just gives medication". However, SP 3 did not know what the doctor or health professionals should have told them apart from the information regarding medication and diet.

In contrast, SP 5 spoke of not being interested in knowing about stroke. She was also not interested in sharing experiences with other stroke patients although she sometimes meets them. Although some participants said co-morbid conditions were discussed, some found the information was very superficial and without sufficient explanation. For example SP 5 was told that she

Had hypertension, I had hypertension, I already know that. I went to the doctor. He asked me to be careful. But, I said, "how to be careful"?

\section{Urutan tradisional and exercise as an important aspect of early stroke rehabilitation}

All the patients used urutan tradisional and other traditional treatments as strategies to achieve optimum recovery. Urutan tradisional was used in conjunction with rehabilitation therapy, and was found to be very important and widely used during the early rehabilitation of the stroke patient. All patient participants stressed the importance of urutan tradisional in their early recovery following stroke. The benefits of urutan tradisional included improved voice production and the asymmetry of the mouth, helping the recovery of movement, and restoring blood circulation. 


\section{(i) Multiple benefits of 'urutan tradisional'}

Participants SP 1 indicated that urutan tradisional was useful for improving his voice production and the asymmetry of the mouth. This participant also learnt a little bit about stroke from the massager.

The massager said that the blood vessel at the neck and the voice ... he did the massage and it's become all right. Then, the voice was improved. The mouth became symmetrical which was asymmetrical before.

Apart from improving voice production, urutan tradisional was also found useful to improve mobility

When discharged, four days after discharge, we use massage. While in the hospital, the next day, we took the traditional massager to the hospital, doing massage at the hospital. Then, when discharged ... we use 'urutan tradisional' everyday. There is an improvement everyday, able to move and lift hands and feet. After three weeks, [I'm] able to walk, produce voice, able to talk, but with slurred speech. (SP 1)

Similarly SP 4 said

Once discharged from the hospital, I stayed at home with mom. She did everything. Prepared and served meals. Then, when I stay here, I started falling ill again and was admitted to the hospital for two weeks. Then discharged, come back here, so able to call people to do massage. When used 'urutan tradisional', was able to sit up and then walk. Until today, do not need to use walking stick.

The traditional massagers not only gave massages but they also gave encouragement and motivation for patients related to their recovery. For SP 5 it was the support and encouragement of the massager that aided her mobilisation.

We do massage, then after four times, the traditional massager asked me to try to walk. I said that I cannot walk. He [massager] has to watch me. He asked to try again, he lift. I said, "Watch me, otherwise I will fall”. "Walk ... walk ... yes, you can".

Once the patient had shown him she could walk he advised her "to walk every morning". Participants also spoke of other physical benefits of urutan tradisional. For patient SP 1 
this benefit concerned improvements in circulation. SP 1 talked about how the massager had

Touched and pinched the blood vessels/nerves. Stroke strongly affects the nerves or blood vessels. If we are lacking of 'urutan tradisional', it will cause jammed, so the blood will not moving.

This explanation by SP 1 indicates that this patient also had some understanding about the causation of strokes.

\section{(ii) Frequent and regular use of 'urutan tradisional'}

The frequency of using urutan tradisional varied depending on individuals' preferences and sometimes their financial status. The common practice was to use urutan tradisional more frequently (every day, twice weekly) during the early stage of stroke and to decrease the use over time. SP 1 spoke of initially having a "massage once a week, twice a week ... until now still use massage”, while some others spoke of having it monthly. People referred to the situation where they could not afford to use urutan tradisional because of the cost involved in using it.

\section{(iii) 'Urutan tradisional' and exercise or medicine are equally important}

Two participants believed that urutan tradisional and rehabilitation therapy or medicine were equally important and complement each other. As participant SP 3 stated, "I am recovered now because of the combination of medicine and 'urutan tradisional'. How they complement each other was described by SP 1 who said during exercise it was "follow this" whereas with urutan tradisional the focus is on "looking at the blood vessel/nerve underneath". Participant SP 1 also expressed that urutan tradisional and exercise have their own function in stroke recovery.

Traditional healer, he play with the nerve/blood vessel by "cokeh" [digging], move this finger, and he "cokeh" other nerve/blood vessel, then can move the finger, that's why we use both [urutan tradisional and exercise]. Otherwise, now the nerve/blood vessels already died, without effort the nerve/blood vessel had died.

All interviewed patients use and believe urutan tradisional is an important intervention during the early stroke rehabilitation. However, one of the participants (SP 2) felt there 
were limited effects from urutan tradisional. He said, "I feel that my body is weak ... but not much effect [from urutan tradisional]”.

Two other activities were referred to that had been used during the early stage of recovery. These were the wiping the body with embun (dew) or lying on top of it and covering the body with sand at the beach. Embun is the water that normally can be seen on the grass during the very early morning. Most patients expressed and believed that using embun was an important strategy to use for recovery to make the body and muscle become tough. Participant SP 4 said she "put 'embun' and wipe all over the body, some people lie on it, but I can't do that because of itchy”. Participant SP 2 mentioned "I also went to the beach and was under the sand for about one hour. This was informed by our old people”. Getting information from other people such as elderly and friends is also found common among patients and their families.

\section{The impact of stroke}

(i) On physical, social life, behaviour and emotion of stroke patient

Following their stroke, all the patients talked about the rapid changes that happened in terms of behaviour, emotional and physical. These changes had a negative impact on their everyday life. Participant SP 2 said "When at home, I do not go anywhere because I could not go by myself and must be dependent on other people". Patients also expressed that changes in their functional abilities and not being able to socialise as before stroke led them to feeling isolated and created low self-esteem. The effect of stroke such as weakness and lack of energy experienced by all of patients also led to social isolation as captured by SP 1

It is hard, because stroke patients are not many, sometimes I feel isolated because of being alone, other people are all normal. When being alone like this, we feel low self-esteem; stroke people ... most of them feel low self-esteem.

This patient was isolated due to weakness and being unable to go out by herself. The fear of stroke recurrence and crossing the road was expressed by participant SP 5. This partly added to her social isolation. Having a stroke and perceiving herself as different from other persons also led to social isolation. As SP 5 said 
I feel weak, so how to socialise or enjoy with other people ... I already had stroke...unable to go out, unable to get fresh air, just sit quietly in the house, uninterested to talk.

The feeling of isolation in the house expressed by SP5 was not raised at all by SP 4 . Participant SP 4 was more concerned about being unable to fully function after having stroke.

Three participants talked about the behaviour and emotional changes after stroke. Two (SP 2 and SP 3) found that they had become more sensitive. In addition, SP 4's husband mentioned that

I have to really look after her very gently, give loving care. Other stroke patients who have died also told me about this and to avoid pressure. The doctor also told me.

On the other hand, SP 3 realised the consequences of anger and stress on a stroke patient. From his point of view, stroke patients needed to be more patient in dealing with the behaviour or emotional changes caused by stroke.

People who have had stroke ... must be more patient, cannot be easily angry ... must be more patient. If we always angry ... it will be vary hard ... because we've got this type of disease.

\section{(ii) Work changes after stroke}

In general, all five patients described about lack of energy, weakness and less concentration to do their previous job. This condition caused them to do less heavy work such as gardening and to work in and around the house. The three male patients all expressed not being able to do their previous job due to lack of energy. Participant SP 3 shared that it is very hard for him to explain how he had a great loss of strength after the stroke.

There is a lot different. Before, [I] used to be strong, but now, not strong enough. That's the difference. And, unlike before, I am not able to really do the work as a builder. 
Similar issues of having a lack of energy were commented on by all other four patients. A lack of concentration after having a stroke caused participant SP 1 to have less interest in doing his previous job although he is capable to do it, as expressed by his wife. "After stroke, so ... he ... did not go to work, he does some work in the house, does some gardening, and cleaning around the house, so to make him sweat". Participant SP 1 also added "I do not know, because I'm not well, so ... no attention to do the work".

\section{Coping strategies or dealing with the impact of stroke}

One of the four Muslim stroke patients mentioned that she recites the Holy Qur'an every day as a source of help and coping strategies. As expected, for Muslim stroke patients in the study reciting the Holy Qur'an to cope with the consequences of stroke was important. Stroke patient SP 4 said, "Always recite the Holy Qur'an ... we try to remember what we recite". Belief and acceptance on what was given by God was also expressed by participant SP 5 who is non- Muslim. This might be one of the ways to cope with the impact of stroke. However, this situation also led to a lack of interest and motivation in her everyday life.

I feel like having fever, because of the nerve. [I] feel weak, I just sit quietly. I am not interested to talk with other people. In this house, if my grandchildren made a joke or something funny, sometimes I feel that I want to laugh. In term of talking, I don't feel like doing that, let it be ... because God give me this.

(ii) Avoiding stress and promoting socialisation as a coping strategy, to prevent stroke recurrence and promote recovery

The majority of stroke patients were aware of the possibilities of recurrent stroke and the contributing factors towards it. Participant SP 1 indicated that the two main ways of preventing stroke recurrence are through food and by avoiding having problems with anger and stress. Stress and social isolation also might delay recovery. According to SP 1 foods that contain high cholesterol or fat may cause stroke recurrence. However, problems causing stress were considered a greater risk in having stroke recurrence compared with food. 
To prevent stroke recurrence is through food, we have to control it. But in terms of problem, it is number one. It is number one than the food. So, food is number two....

Avoid problem, because when facing problem, suddenly will get another stroke.

His wife added, "Problem is the most important ... because ... when he is facing problem, suddenly he will turn to unconscious".

\section{Services and information or education received}

Health services received by stroke patients prior to discharge and while at home varied. One of the patients (SP 1) who was under a research programme received regular rehabilitation therapies. Three other patients were never referred to a rehabilitation unit for rehabilitation therapies. On the other hand, transport problems to attend follow-up visits at the rehabilitation unit were also raised by SP 4 .

During three days in the hospital, the only information and education received by SP 2 was that he was asked to take the medication and to come for rehabilitation therapy. "The doctor just said ... come for therapy, and then prior to discharge, the doctor said ... come for follow-up visit. Must come ... then he did not say anything else." No information related to stroke was given. In contrast, participant SP 1 was taught to do exercise at home. "He [rehabilitation therapist] demonstrates ... the exercises that need to be done at home".

Overall, there are inadequacies in the provision of information for stroke patients. Patients also showed they did not know what to ask from health professionals. As participant SP 4 indicated she did not have any idea what the doctor would ask her during the follow-up visit because the doctor never asked her. And she too never asked anything.

\section{Support need}

In general all the five patients expressed the need for support at home during the early stage of rehabilitation. Participant SP 2 talked about the support needed in doing the 
ADL such as toileting and eating as she said, "If I am able to manage self, for toileting. It is hard [for caregiver] to manage [to take care of me] alone. I also find it difficult to eat".

Some patients commented on feeling lazy and always wanting to sleep. This affected their progress in carrying out rehabilitation activities such as physical exercise. Continuous support from family members especially the primary caregivers was needed to give motivation and guidance to perform physical exercise. As SP 1's wife mentioned, Other people [eg: spouse / caregivers] need to always be with him [patient], so ... to help him, remind him, ask him to do exercise, ask him to do the job that he is able to do it, then only he could have some feeling.

She also added,

Just like no motivation. If he was left alone, he would feel weak and wanted to sleep. After eating, he wanted to sleep, that's his only job. He couldn't think of others. So we need to teach/train him, inform him.

Emotional support also needed to overcome the feeling of inferiority and low self-esteem as well as to promote socialisation.

Two of the five patients suggested that the healthcare service or government should organise programmes such as sport for stroke patients. This would mean that patients who are from different places and living far from the area would be able to meet each other. Participant SP 1 said

Government should organise ... like ... where people meet, just like other disabled people ... they organise a sport for disabled people.

\section{The benefit of meeting other stroke patients}

Meeting other stroke patients was found useful for patients because there are always other patients who are in a more serious condition. These conditions made patients feel better about themselves as they felt fortunate compared with others. As SP 1 said 
At home, I did not meet other stroke patients. But, when I go to the hospital, I feel much better because other patients are more serious. If I stay at home, I feel worst. But when I see other stroke patients ... I feel better and happier.

Meeting other stroke patients was also believed as a source of information on stroke as well as about stroke experience. Participant SP 4 expressed that "I want to know, want to know about the disease". However, she had no opportunity to talk with other stroke patients because of her inability to go out by herself. As SP 4 said, "[I] want to meet other stroke patients ... but they are not around, "[I] had no opportunity to talk, and I also cannot go".

In addition, meeting other stroke patients gave them the opportunities to share experience as well as to avoid tension and boredom as mentioned by SP 2,

It is good to go there [rehabilitation unit], to avoid tension and boredom .... Able to talk ... have conversations .... I also asked them [other stroke patients]... their strategies/ method ... asked them ... how to recover faster... Yes, can meet them [other stroke patients] ... as a guidance.

\section{Hoping for the future}

Of the five stroke patients, four had positive attitudes towards recovery. As mentioned, SP 2 expressed his desire to manage himself and to recover faster. He was also really keen to drive again and return to work. He talked that driving is one of the ways to promote socialisation and to improve self-esteem of stroke patients. Compared with the older stroke patients, SP 2 as a younger stroke patient was the most positive and enthusiastic about his future life.

\section{Findings of the caregiver interviews}

The same procedures for recruiting patients were applied for family caregivers. This means that patients and family caregivers were recruited concurrently. During the whole process of approaching patients and family caregivers via telephone, the first person that was approached was the caregiver. The five family caregivers' transcripts were 
identified using the terminology CG 1 to $\mathrm{CG} 5$. One of the caregivers, CG 5, is the spouse of patient participant SP 4.

\section{Caregiver participants}

Table 4 shows the socio-demographics of the family caregivers of patients who were interviewed. All of the caregivers were Malays. Four of them were female and have a close relationship with their stroke family members such as a daughter and wife. Only one of the caregivers was male, he was the husband of one of the patient participants. From Table 4 it is apparent that four of the caregivers were from the moderate education level having completed secondary school, and one had academic qualification at first degree level. All of them were of at the working age, 43 to 58 years old. Four had three to five children and one of them had 12 children. All of the caregivers were living with at least one other adult family member in the house.

Table 4. Socio-demographics features of family caregivers

\begin{tabular}{|c|c|c|c|c|c|c|}
\hline $\begin{array}{c}\text { Partici } \\
\text { pant }\end{array}$ & Age & Gender & $\begin{array}{c}\text { Education } \\
\text { level }\end{array}$ & Occupation & $\begin{array}{c}\text { Relationship } \\
\text { with patient }\end{array}$ & $\begin{array}{c}\text { Marital } \\
\text { status }\end{array}$ \\
\hline CG1 & 48 & Female & $\begin{array}{l}\text { Completed } \\
\text { secondary } \\
\text { school }\end{array}$ & Housewife & Daughter & $\begin{array}{l}\text { Married with } \\
12 \text { children }\end{array}$ \\
\hline CG 2 & 49 & Female & $\begin{array}{l}\text { Completed } \\
\text { secondary } \\
\text { school }\end{array}$ & Housewife & Wife & $\begin{array}{l}\text { Married with } \\
\text { four children }\end{array}$ \\
\hline CG 3 & 43 & Female & First degree & Teacher & Daughter & $\begin{array}{l}\text { Married with } \\
\text { three children }\end{array}$ \\
\hline CG 4 & 58 & Female & $\begin{array}{l}\text { Completed } \\
\text { secondary } \\
\text { school }\end{array}$ & $\begin{array}{l}\text { Housewife, } \\
\text { earning side } \\
\text { income by } \\
\text { selling } \\
\text { morning food }\end{array}$ & Wife & $\begin{array}{l}\text { Married with } \\
\text { five children }\end{array}$ \\
\hline CG 5 & 44 & Male & $\begin{array}{l}\text { Completed } \\
\text { secondary } \\
\text { school }\end{array}$ & Labour & Husband & $\begin{array}{l}\text { Married with } \\
\text { four children }\end{array}$ \\
\hline
\end{tabular}

The caregivers were interviewed at various places including their home, workplace and the interviewer's house as agreed by both of us. The interviews ranged from 25 minutes 
to 100 minutes, with an average of 58 minutes. Four of the interviews were conducted solely between interviewer and interviewee without the presence of other family members, and in the fifth interview, the caregiver was accompanied by his stroke wife, SP 4. The same procedures with stroke patients' analysis were applied for the caregivers.

\section{The themes}

Six major themes emerged from the family caregivers' analysis.

1. Strategies for recovery

2. Information and education needs

3. Understanding of stroke and post-stroke care

4. Support needs

5. The impact of stroke on the caregiver's life

6. Coping strategies/dealing with the burden of care giving

The findings of each to these main themes are presented separately

\section{Strategies for recovery}

(i) 'Urutan tradisional' as an important aspect of early recovery

All the caregivers talked about the importance of urutan tradisional. One participant, (CG 2) felt that urutan tradisional was really important for recovery. She said, "Twice a week or once a week we use 'urutan tradisional'. Must use it regularly, must use it." Participant CG 3 provided more information about urutan tradisional.

We do massage at home. For the beginning, he [traditional massager] do massage three times, twice a week. Each massage takes one hour, one and half hours, it is quite a long time.

Participant CG 2 added that the use of massage was encouraged and supported by health professionals. According to CG 4, massage was used while her stroke husband was still in the hospital.

We asked for... like ... because no physiotherapy, must use 'urutan tradisional'. Traditional massager did like what physiotherapist did. Because, while in the hospital, I already take the traditional massager to the hospital and shown to the doctor. Because, I cannot do it, do not know how to do it, because I do not know 
about the nerve. Then, the doctor said, doesn't matter. He [traditional massager] went to the ward, once a week.

Indeed, they use 'urutan tradisional' at the very early stage even the day after the onset of stroke before getting the hospital treatment. As participant CG 1 said,

We did not take her [mum] to the hospital [after the stroke] ... the next day only my sister take her [mum] to the clinic $K$ [private health clinic], then she call for 'urutan tradisional.

One participant mentioned that 'urutan tradisional' and physiotherapy were equally important and need to be used concurrently. As CG 3 said

Actually, 'urutan tradisional' and physiotherapy is almost the same. Massage is more to the nerve, internal, whereas physiotherapy is more to the movement. They must be together. They cannot be alone.

None of the participants from caregivers and stroke patients expressed any concern or worries about the risk of traditional massage. One of the caregivers, CG 3, also used spa and sauna for her stroke father as a rehabilitation activity.

\section{(ii) The use of 'bomoh' (traditional healer)}

Apart from treatment provided by the hospital, a bomoh was also used to assist in recovery from stroke. In their practice, bomoh used various methods to treat patients. According to CG 1, her mother used a bomoh twice to assist with recovery: "take her [patient] to the 'bomoh's' house”. Besides that, a bomoh was used to assist in recovery and well-being of the patient. S/he believes that one should take the initiative and effort and use various kinds of strategies for recovery. As participant CG 1 said

S/he [bomoh] said ... you [caregiver] have to take a lot of effort ... because your mum [patient] used to give bath [wash before bury] to the dead body. You know that may be something would be inherited from that. Then I invite the 'bomoh', s/he said, if we didn't remove that things will make it hard to die. I thought about that, and then maybe ... yes ... in case something happen, we do not know. Then I take her [mum] to the 'bomoh', twice ... to get rid of all those things. Then, when she went home, I and my aunt who came to visit asked her condition, and 
then she [mum] said, "Feel much better". She was not in pain like before. I took all the efforts in many ways ... follow the hospital treatment as well as this method.

The bomoh also provided air tawar for patients. Air tawar is water that was used by the bomoh in various ways depending on the individual bomoh. Some of the bomoh performed solat hajat (hajat prayer) and placed bottled water in front of the praying area. Others may recite a specific versus from Holy Qur'an in front of the water. For example, air tawar can be used to assist in recovery by drinking it or using it to wash the patient's face, or for mixing with other water for having a bath. In relation to this, participant CG 1 said, "I give ['air tawar'] to mom to wash her face and to drink it ... can open the eyes ... while $[\mathrm{mom}]$ in the hospital".

\section{(iii) Food and other traditional strategies}

Caregivers have made various efforts and used strategies to promote and facilitate stroke recovery. Of the 10 interviews with stroke patients and caregivers, one participant highlighted the importance of eating eel for recovery. She said, "[eat] a lot of eels. I make eel soup. Sometimes, eat eel everyday. I asked the dealer to cut it, sometimes, I do it myself" (CG 2). Eating eel was considered important because "it could recover our nerve. Our energy will recover. Our nerve could heal". Two other caregivers used embun (dew) as a method to promote recovery.

I buy small stool for him. Take him [husband] there [to the garden], because 'embun'... embun' will speed recovery [healing].

(iv) Psychological need

Psychological healing was also believed very important for stroke recovery. Similar to the findings from the patient interviews, all the caregivers believed the importance of psychological support for patients. And one of the ways of getting this is getting attention from other family members. As participant CG 3 expressed

Currently, I think my dad would recover if his psychological need also healed. Now, the problem is psychological. His physical condition is getting better. I wish that my dad would recover if he gets attention from his children. 
The above statement was supported by the similar statements by CG 1, CG 4 and CG 5 . Stroke patients need to prevent having any problem or listening to bad news because it would aggravate their condition, as mentioned by CG 2 .

You know ... stroke patients, we cannot tell strange news to them, cannot. We should always tell them good news. If we are having a problem or do not have enough money, we cannot tell them. If they want to eat something, we have to try to give them. Do not let them think about the problem.

CG 5 also added that caregivers should not let stroke patients become upset.

We [caregivers] should advise stroke patient to take care of the diet and enough sleep. And then be gentle to them [stroke patient]. If s/he tell something, listen to him/her. If s/he tells us, listen to them, but do not scold him/her back.

CG 5 shows some awareness and understanding that caregivers should provide emotional and psychological support to their stroke family members. This knowledge was obtained from other people in the community who had experience in looking after stroke patients.

(vi) Prayer, reciting 'Holy Qur'an' and drink 'air zam-zam' (Holy water)

Two of five caregivers talked about the importance of prayer and reciting the Holy Qur'an as one of the ways to get help from Allah for recovery. As CG 2 stated

The most important, I asked him [patient], to always remember our messenger of Allah, always remember Him [Messenger of Allah]... Ask for help from Allah. Allah will heal his disease /recover him from stroke.

Apart from prayer, participant CG 4 also provides an opportunity for her stroke husband to listen to the Holy Qur'an through a cassette player and gets him to drink air zam-zam. This is part of ritual activities among Muslims, it also fulfils a psychological need.

We provide him with ablution. Then I will tell him that the ablution has been done. Then I switch on the cassette player of 'Holy Qur'an'. He may follow. Because of he may not sleep at night. It is better for him to listen to the 'Holy Qur'an' rather than doing or dreaming about something nonsense. 


\section{Information and education needs}

(i) About stroke

Caregivers also expressed the need to know about the nature of stroke, the causes of stroke and how stroke occurs, prevention of stroke recurrence and where to get information on stroke. Participant CG 5 expressed that he has never been given any information on the prevention of stroke recurrence. When asked what they understood about stroke, CG 5 quickly said that there is one thing that he wants to know. "I want to know how stroke occurs and the causes of stroke."

And again when asked where he can get the information about it, he added

I want to know a lot more ... whether caused by food or from something else ... or caused by hypertension ... or ... what are the causes of stroke?

Participant CG 4 wanted to know about her husband's condition and something related to nerve because stroke will involve nerve.

About his [husband] nerve, you see ... when he sleeps, he cannot strengthen his leg, his leg was moving up and down.

She added that she required information on how to make her husband comfortable. " $I$ want to know about how to care for him ... to make him comfortable. I noticed that he always lies in an improper position, you see."

The need to know about all aspects of stroke including medication was also expressed by CG 4. "Want to know all, and related to medication. What is the right medicine?"

Nearly all stroke family members were given no information on how to assess the progress of stroke patients at home, but they used their own method or ideas on how to assess stroke patients at home. As CG 5 stated

We observed her everyday, we see how she walks, let's say, today like this ... then the next day we can see the changes .... I think, the most important that ... stroke patient ... in term of advice to stroke patient, if possible, I want her ... her weak arm to play with something.

When asked about the information or education given before discharge, CG 5, he stated that the doctor just told them to come for exercise (physical therapy) at the rehabilitation unit, but no appointment was given for that. 


\section{(ii)Management at home}

In relation to the management at home, some caregivers did it based on their own ideas. Some got the information on how to care for stroke patients from other people who came to visit them. "No, we did not ask [other people], but they told us about that and other people told us about this, so we get some information." The majority of caregivers expressed the need to know how to manage stroke patients at home in many areas: suitable food for the patient, communication, positioning, home exercise and safety items. CG 3 talked about the need to know the proper way to interact with the patient and the basic exercise that can be done at home.

I think, first of all, how to interact with patient whether soft or firm so that s/he did not feel hurt. When we made a comment on a certain thing, s/he can accept it. If possible the way how to interact. The second one is the safe exercise, simple exercise that we can teach.

Another caregiver (CG 1) also wished that someone would tell her how to care for the patient at home.

In terms of the food or nutrition for patients, two of the five caregivers mentioned wanting information about the right food that should be given to stroke patients. As CG 3 said, "the food that is suitable for him [her stroke father], that's all I think". CG 1 also mentioned

So, we want to know, what type of food for the morning, afternoon, evening and dinner for this type of patient .... Need to know about nutrition, therapy technique ... I want to give motivation to her.

\section{Understanding of stroke and post-stroke care}

With regard to the understanding of stroke, the level of understanding of stroke and issues related to stroke varied amongst the caregivers. Participant CG 1 had some understanding and ability to recognise some of the effects of stroke.

Stroke is ... I do not understand ... is ... an illness ... that unable to sit up [stand up]. So ... the mouth turned to asymmetry. If the mouth deviate to the right ...it 
means that the left upper and lower limb become stroke ... right? ... then right upper and lower limb able to move.

In contrast, CG 5 could not give any comment about his understanding, how stroke happens or the causes of stroke because he does not know about it. For CG 4, it seems that there is some understanding on stroke while she talked about an association of stroke with diabetes. From her understanding, stroke happens because of the increasing level of diabetes as well as being caused by smoking. "I understand, because he [husband] had diabetes, so, because of the increasing level of diabetes. And then he was also smoking".

Participant CG 3 who is more educated compared with other caregiver participants has more clear understanding about stroke.

From my understanding, stroke involves the brain, and the inability of the brain to provide information to other parts of the body to react. And it is not a permanent disability. Stroke can be cured if it is caused by the blood clot and also depends on the severity of the stroke.

To CG 3 stroke can be cured; however, there is no medication that can directly cure stroke. It is more on how stroke patients handle it positively. The association of stroke with the brain, nerve, paralysis, blood clot, interruption of the blood flow and the air in the body was expressed by caregiver CG 3 .

To me, stroke can cause paralysis ... and the air ... the nerve ... do not work well, and the blood clots in the blood vessel, it is not active [blood flow] all over the body.

She added

Blood clot all over the body. To me, even though stroke happens from waist downwards, he [husband] still fell down.

This participant did not agree with what the doctor told her about her husband. According to the doctor, her husband had stroke of the brain, but to her it was not the brain because her husband's memory was still quite good.

Yes, the doctor said that he [husband] had stroke of the brain .... But if stroke of the brain, it is hard to recover. But to me, he [husband] does not have stroke of 
the brain ... if he had stroke of the brain, he would not remember... and he would not be able to drive in just 10 months.

However, she did understand about the cause of her husband's stroke.

May be his nerve ... for sure his [husband] nerve is not damage. May be his blood ... his blood has clotted, from the neck upwards ... may be the blood vessel was blocked by the blood clot.

\section{Support needs}

All the interviewed patients and caregivers live with at least one other adult member in the family. Apart from looking after patients, the caregivers also had responsibilities for other family members especially their own children and the household chores. Due to the hard work in managing patients at home, all of them expressed the need for support from other family members to reduce and share the burden of caring. As expressed by CG 3

One of my problems is...because my sisters did not help me, that is why I felt very sad. They think...everything was left on me, because I am the youngest, I haven't got the guts to say, "please come and take care of dad for a while because I want to go out".

She also added,

That's my problem. My sister, she takes for granted that I can look after my father on my own.

Apart from the need for support from siblings, all the caregivers spoke of getting help from their own children.

We [caregiver and her children] take turn to look after dad. In the morning, my son [13 years old] has to look after his grandpa [patient], give him food and medicine, because he knows about medication.

CG 3 added that she really appreciated the support and help from her family, otherwise she would not cope to look after her father as she also works fulltime.

There is a lot of difference. Thank you to my families because they have sacrificed a lot. Sometimes I need to give motivation to my children by saying that, "If I'm alone, I cannot cope, I also work, and all of us have to take care of 
grandpa". The only reason that I give is that "we only have one grandpa". My children can accept it. Thanks to God, my husband and my children are very supportive.

To assist patients in performing prayer five times a day is not an easy task. Due to the many other commitments for the caregivers who are on full-time work, sometimes it is quite a challenging task to provide assistance with prayer. Participant CG 3 talked about how her stroke father had scolded her because of delays in assisting him to pray, "Don't you understand ... that I am not praying yet ... don't you understand that I am not praying yet". On other occasion, when feeling too tired, her father would comment that sick people like him do not have to pray. Participant CG 3 considered she was always aware of her responsibility to assist patient to pray by encouraging and reminding him to pray. From her point of view, as long as her father can hold food and put it into his mouth, he must pray.

Assistance from family members was considered important in relation to activities related to prayer. Taking ablution is compulsory before prayer. Participant CG 4 gets help from her son to take ablution for her stroke husband. During or after taking the ablution (and before finishing prayer) the wife is not allowed to have skin contact with the husband. Therefore assistance from others is very important. As participant CG 4 said, "I cleaned him [husband], and then my children will provide the ablution because I cannot do it”.

Another participant, CG 1, talked about the support needed in terms of finance as well as facilities such as a wheelchair. The caregivers were also concerned whether health professionals were able to provide home visits for stroke patients. This type of programme was considered as very useful and would be beneficial for those patients who have mobility problem. As expressed by CG 4 and CG 5 that transportation is a barrier for follow-up visit.

In addition, the importance of support from a key person in the community was expressed by CG 3. From her point of view, people should be able to be contacted when 
needed especially in emergency situations. Only one caregiver talked about the relationship with the neighbour as being important. Sometimes there is a situation whereby adult family members were not at home and neighbours could be a source of help if needed.

Then, one more thing is we need to build a strong relationship with the neighbours. My father used to fall in the bathroom. No male adult person was available at that time. Therefore my son [who was alone at home] had to call our female neighbours to lift my dad. (CG 3)

Some caregivers would like to have an opportunity to learn about caregiving from more experienced caregivers. As CG 1 said

Because, I want to know from those people who have experience in caring for stroke patients ... because this is my first experience.

\section{The impact of stroke on the caregiver's life}

The impact of stroke on the caregivers varied. It depended on the severity of the stroke, the relationship of the caregivers with the patient and the functional abilities of stroke patients. Of the five caregivers, two of them directly voiced concern about the big impact of stroke on their families and their social life. The dramatic change in family life after her father had his stroke was expressed by CG 3.

Then, when my father became like this [had stroke], my family ... last time we used to have outing days during the weekend, if we want to go out, we can go out at any time.

I cannot deny that, my life had changed now....

Compared with before he had the stroke, there is a lot of difference before we take care of him [father] and after we take care of him. Sometimes, I do not have time to spend with my husband .... My children also do not have normal life ... no ... everything now is very different compared to our life before. Too many factors ... Ifelt very stressful.

The impact of stroke on CG 2 as a wife of a patient was very different for CG 3 . Although having difficulty in caring for her husband with his physical disabilities, the 
emotional burden faced by her was greater than the physical burden. The effects of stroke caused behaviour changes such as he became rude and was easily angry and she noted the lack of an intimate relationship with her husband. This was aggravated by the lack of interaction and communication between them due to communication difficulties experienced by her husband. This condition led to the feeling of loss of a companionship. The marriage relationship was also threatened due to sexual dysfunction caused by stroke.

\section{Caregiving role as a test or challenge from God (Allah)}

With regard to the caregiving role, some participants expressed their willingness and acceptance to take on the roles. They shared the believed that health and illness was given by Allah. One of the caregivers believed the caregiving role was their duty and responsibility as a child. To her, when her mother had a stroke, it was the time her and her siblings took care of their mum.

CG 1

When she become sick like this, I think, this is our time to care for mum ... right? Before she had stroke, when we deliver our baby/in labour ... mum can take care of us, then, she had stroke, it is our turn to look after mum ... right? ... to repay what she had done for us [crying] .... Burden ... I never think of that. Because of she is my mum. (CG 1)

In addition, she did not considered caregiving as a burden but as a test from Allah. Caregivers needed to be more patient.

So ... I would like to tell that if our family member had stroke, the caregiver should be more patient and do not take the caring role as a burden. Do not take it like that ... because it is a test from Allah. It means that Allah wants to test us ... doesn't matter whether our mum, husband, children or siblings ... it means, we have to assume that as a test. We cannot take it as a burden ... right?

\section{Conclusion}

In general, interviews with stroke patients and their family caregivers demonstrated the inadequacies of information and education provided to them on many aspects of stroke 
and stroke care and home management after stroke. Findings also revealed a lack of basic understanding and misconception about stroke among patients and their families. There were also uncertainty by patients and families about what to ask health professionals. And for many of them, they were relying on the health professionals to provide them with the information. The interaction between patients, caregivers and health professionals were usually limited to informing patients to take their medication and to come for follow-up visits at the physician's clinic. Other important aspects of educational needs were related to performing prayer and reciting Holy Qur'an for stroke patients with some residual effects of stroke.

The majority of patients and the caregivers in the study expressed the importance of urutan tradisional for early rehabilitation. And some patients started using it while they were still in the hospital. Others started using it the next day after the onset of stroke. Due to the disability faced by patients, many of them were experiencing problems going to the rehabilitation unit for therapy. They found the traditional massager provided a service at their home which was much more convenient.

Patients and families also used other practices which are culturally and religiously bound for recovery and coping strategies included solat hajat, embun, eating eel, air zam-zam and reciting the Holy Qur'an and assuming that stroke or any illness was a test or challenge from Allah. Besides that, due to the complexity of post-stroke care, patients and families needed a range of support in terms of assisting with ADLs, financial, socialisation and reintegration into the community, emotional and psychological support from other family members, neighbours, community members and the health service. 


\section{Chapter 7: Findings from health professional interviews}

\section{Introduction}

This chapter reports on the findings of the analysis of the 12 health professional interviews. Face-to-face interviews were conducted with health professionals who were working in the hospital, academic nursing staff and with a key member from the National Stroke Association of Malaysia (NASAM). The interviews with the multidisciplinary health professionals covered the early rehabilitation needs at home, the current practice related to stroke care and rehabilitation and the provision of education and information at acute setting and post-discharge and the interfaces between health service and stroke patients and their caregivers post-discharge. The interview with a key member of NASAM focused on the rehabilitation needs at home and NASAM activities and strategies to strengthen the relationship between NASAM and stroke patients and families. This person is referred to as a health professional in these findings.

The detailed procedures for recruiting health professionals were explained in the methodology chapter. All health professionals approached to be a study participant, consented to be interviewed. To maintain the confidentiality of the data, the same procedures were applied for the health professionals as had been used with stroke patients and caregivers. The 12 health professionals' transcripts were identified using the terminology HP 1 to HP 12.

The findings are reported under several thematic headings, primarily using the format of the semi-structured interview guide. The analysis commences with the general background of the study participants. The chapter ends with the summary of the findings from the health professional interviews.

\section{The health professional participants}

Table 5 shows the socio-demographics characteristics of the 12 health professionals who were interviewed. All of the health professionals (except one who was Chinese) were 
Malays. Nine were female and three were male and their ages ranged from 26 to 53 years with an average age of 40 years.

Table 5. Socio-demographics of the 12 health professional participants

\begin{tabular}{|l|r|r|}
\hline \multicolumn{1}{|c|}{ Variable } & Value & Number \\
\hline Gender & Female & 9 \\
& Male & 3 \\
\hline Age group & $26-30$ years & 3 \\
& $31-40$ years & 2 \\
& $41-50$ years & 6 \\
Years of service & $51-60$ years & 1 \\
& $4-10$ years & 6 \\
& $11-20$ years & 4 \\
Education level & $21-30$ years & 2 \\
& Certificate & 1 \\
& Diploma & 5 \\
& Bachelor degree & 4 \\
& Master degree & 2 \\
\hline
\end{tabular}

Ten of the health professional interviews were conducted at the participant's workplace, in the discussion room in the library, one interview was conducted at the participant's home and one at the interviewer's home as agreed by both of us. The majority $(n=10)$ of the interviews were conducted during working hours and were preceded by a general chat. The interviews ranged in length from 34 minutes to 117 minutes, with an average of 58 minutes. The process of transcription and translation from Malay to English was similar to that used with the patient and caregiver interviews.

\section{The themes}

Nine major themes emerged from the health professional analysis.

1. Inadequacies of information for patients and families

2. The important aspects of early stroke rehabilitation

3. The benefits and risks of urutan tradisional

4. Lack of knowledge among health professionals about the role of the rehabilitation therapist

5. Educational needs of stroke patients, family caregivers and public

6. Support needs 
7. Religious activities as an important rehabilitation therapy

8. Caregiving role as a test from Allah

9. Health professionals' perception of stroke rehabilitation.

\section{Inadequacies of information for patients and families}

Analysis of interviews with health professionals elicited similar findings to those of the patients and caregivers with respect to the provision of information. Participant HP 1 talked about the lack of provision of information for stroke patients. This participant considered that the focus of the medical professionals (doctors) was usually limited to the patient's medical condition. As HP 1 said

There is lacking in the provision of information ... just give medicine, then discharge. When the BP is stable ... patient is discharged.

It was also thought that doctors would only give information when asked.

If doctor wants to give [information], they will only give brief information. Then if patients do not ask ... how long ... because not many doctors would like to inform patients' progress. If the relative did not ask, they [doctors] will not inform. I also do not know ... why.

It was recognised by all health professionals that the majority of stroke patients need continuity of care after discharge. Therefore the provision of information and education prior to discharge is important to facilitate patients' recovery. In relation to this, HP 9 expressed her frustration with current practice of provision of education.

From my point of view and my experience, education prior to discharge is very frustrating ... for example ... [staff] do not have the standard plan/schedule that ... when ... stroke patient came in [admitted] and at a certain period, we [staff] have to teach them.

In relation to this, HP 11 said, "In terms of information provided, it is quite lacking. Doctors only talk during the ward round and explain ... what to do". Participant HP 4 also commented that limited information was given to family caregivers in the ward. However, families were often asked to help with the activities of daily living (ADL). " $I$ think, while in the ward, not much that [information] was given ... because what we give ... usually we asked relative to help us with ADL." To HP 5, the involvement of 
families in the care of patients while in the ward is important preparation for the families to care for the patients at home. This involvement helped families learn how to manage activities such as lifting, feeding and washing.

Some of the participants also mentioned the discharge preparation. "No discharge preparation. If we said that 'no preparation' we will be scolded" (HP 3). Two participants also commented that the provision of information largely depended on individual nurse's beliefs and attitudes, as well as their status of education. The provision of information prior to discharge is not common practice. Participant HP 3 said

Nurses will decide ... if they [nurses] have the knowledge, they will give explanation to patients and their caregivers. If they do not have the education, they will leave patient without being given information. They will not do it [providing information].

In relation to this, participant HP 9 suggested that they need to have a team whose primary responsibility is discharge planning.

It means that we ... do not have education plan /schedule prior to discharge. So far we do not have that ... which means that it was given informally. If the staff want to inform, she/he will inform it. If the staffs don't care, don't want to inform ... or busy whatsoever, it is nothing. So we need to form a team.

Having no standard education plan and a lack of time for nurses to provide education, added to the overall lack of information. The provision of information was mainly based on informal methods as expressed by HP 5.

Mostly we provide informally, while we give bed bath. We do not have enough time to give education in formal method. Three staff need to look after 40 patients. Sometimes they [staff] do not have enough time to eat. That's all I can say.

Participant HP 3 suggested, "We have to immediately inform the caregivers about stroke. First of all, to know about patient's condition ... tell them about stroke until they understand". Indeed, HP 3 further suggested that an education unit should be formed to teach the nursing care to the caregivers. "No, we do not have [education program for 
caregivers] at this hospital. So, we need to have a unit, a unit that can provide education on the nursing care”. However, participant HP 4 expressed that there was a limitation in terms of staffing and heavy workload if all staff were to inform patients. From their point of view, one nurse should be responsible as counsellor to provide education for patients who are going home.

Some participants gave suggestions on the timing of education. Participant HP 5 suggested that apart from the education provided prior to discharge, staff could provide education during follow-up visits.

They [clinic staff] can conduct a programme; give health education to the caregivers. Before meeting with the doctor, one nurse could be in charge, gather all stroke patients and their caregivers and give education and information.

Participants recognised that the recovery and rehabilitation of patients involves a lot of physical activities, therefore caregivers should not only be equipped with knowledge but also with skills in managing ADL. As HP 7 expressed:

They [patients] should not hold themselves back, very important and also ensure that they have a support system and that is among their families, that they understand how to transfer patients ... how to get around in a wheelchair, to help accommodate them in a situation.

\section{The important aspects of early stroke rehabilitation}

(i) Prevention of complications

The majority of health professionals commented on the importance of prevention of complications such as pressure sores, pneumonia, and contracture/stiffness and shoulder subluxation by taking care of the joints, proper positioning and regular turning and encouraging mobilisation. They also talked of the importance of education in the prevention of complications during stroke rehabilitation. As noted by HP 11,

At the early stage is to prevent long-term complications such as bedsore because patient is unable to mobilise. Bedsore will increase patient's mortality.

Participant HP 10 also discussed bedsores. 
So, I emphasised on the patient's skin so that patients who are paralysed will not get bedsore/pressure sore. We advised patients' families, we teach families on how to change position ... also give attention on the bed sheet to prevent creases. Bed sheet creases can cause pressure on patient's skin and can cause pressure sore.

In addition, HP 10 and HP 12 described the need to prevent "inflammation of the lung" or "pneumonia" by not letting patients be static or letting them sleep all the time.

Appropriate positioning was considered to be very important during the early stage of rehabilitation. Patients needed to try various positions and pay attention to the affected arms or limbs to minimise complications. Poor positioning may cause complications such as pressure sores, stiffness of the joint and muscles and shoulder subluxation.

Participant HP 2 found that some patients were prone to stay in the same position for a very long time, and that this could result in the development of stiffness and deformity.

For those [patients] who sit on the floor were unable to get up end up with deformities. For example for those who sit on the floor till become stiff as she [patient] said, "I never get up" then as she [patient] said that she moved by moving her body while sitting.

Health professionals highlighted about the importance of the care of joints to prevent stiffness. This can be done by doing regular exercise (at least three times a day). Apart from exercise, proper positioning of the affected arm or limb was also considered important to help in the care of the joint. One of the health professionals, HP 10, thought that care of the joint was the most important factor in the early stage of rehabilitation.

First of all, the most important is taking care of the joint, the limb such as shoulder, elbow, arm, fingers, thigh, knee and ankle. If these joints were immobilising, it may cause stiffness of the joint and muscle.

HP 1 talked about the consequences of poor positioning.

At the early stage ... positioning ... discharged stroke patients ... usually, stroke patients that had been referred to me ... while at home, patients were not attentive on their hands and the position they sleep. This would worsen their 
condition, would increase the tone ... then when they came to us and already fit for outpatient, their condition was even worse.

\section{(ii) Caregivers'awareness}

Three of the health professionals (HP 1, 2, 3) stressed the importance of caregivers' awareness and education on the home management of patient post-discharge. Family caregivers were considered to play major roles in promoting patients' motivation and assisting the rehabilitation activities. According to HP 1

Caregivers' education is very important ... because stroke patients ... they know that they can't do ... so they said, "I can't do, I want to sleep, and I can't do it". It is hard. Patients always lack of effort ... not interested to walk ... We need to really brainwash the caregivers. We have to give education to caregivers ... important, very important.

To my opinion, at the early stage, caregivers should have awareness, about ... treatment and the management of stroke patient after discharge. Because I used to came across many caregivers who do not know what to do. After discharge, they just ignore the patient. Actually ... while in the ward, for example, when the doctor referred a stroke patient to me, then I will teach on how to prevent complications. (HP 2)

Health professionals were concerned that the majority of patients refused or were uninterested in doing rehabilitation activities. Patients were sometimes perceived as 'lazy' and not motivated by their family caregivers. In relation to this, HP 10 believed that the lack of motivation might be due to the side effects of drugs.

Because there is a lot of misunderstanding, when I have a discussion with families, they told that patients were lazy, don't want to do it [exercise]. That's not the real reason. This is the effect of drug. When that particular drug is stopped ... that kind of attitude [laziness] disappears. This is important, I don't want to give the name of the drug, but for sure the antihypertensive drug will affect patients' physical performance. Patients become inactive, confused, and sleepy and want 
to sleep. This situation is interpreted by family members as the patients were lazy, useless, unmotivated and refusing to do exercise.

Participant HP 10 also suggested that studies should be done to explore the effects of certain drugs on patients' motivation. The importance of encouraging patients' motivation during the early stage of rehabilitation was also expressed by participant HP 11.

Sometimes patients were not motivated. It is important to give motivation to patients and their families. Usually patients get mentally depressed. Therefore it is important to treat patients' emotions so that they will have strength to carry out rehabilitation activities.

\section{(iii) Someone in charge of patient at home (primary caregiver)}

Two participants, (HP 3, 12) highlighted the importance of ensuring that someone would be in charge to look after the patient at home.

And the role of the nurses ... while the patient is in the ward, to teach family member ... who will look after the patient when s/he [patient] discharged home. (HP 12)

Another important aspects that would make a critical difference to the health and wellbeing of stroke patients at home included staff appreciating the conditon of the house, assessing the level of the patients' disability because this would determine her/his independence, and assessing the caregiver's willingness to participate in caring for patient .

Participant HP 3 also stressed that early caregiver participation in the care of patients at the acute stage to promoted optimum recovery.

For example, we [health professionals] have to explore how far is the caregivers' participation in caring for stroke patient, if he/she [caregiver] already involved, patients would be safe. On the other hand, if caregivers did not participate, no one would help her/him [caregiver] when patients returned home, then patients would be left unattended. 


\section{(iv) Attitudes}

Two of the health professional (HP 8, 12) talked about people's attitude as an important aspect of early stroke rehabilitation. Three categories of attitudes were identified: therapist, family and patient attitudes. Therapist attitudes refer to how rehabilitation therapists should behave while they were visiting patients' homes. "Therapist should be aware that they are a guest" (HP 8), and they need to be aware of possibilities of discussing sensitive issues in relation to aspects such as house modification. Any suggestion for house modification should take into consideration the status of the house, whether rented or owned by patients.

Family attitudes were related to caring responsibilities. Health professionals acknowledged the physical and emotional burden of caring for stroke patients. Therefore family members should understand the stroke condition and the effects of stroke on patients and health professionals should have some ideas or alternatives to lessen the burden faced by family caregivers. This is particularly in relation to the activities of daily living (ADls) such as transferring or lifting patients.

Participant HP 12 discussed the experience of family members who are reluctant to take their stroke family member home due to no one being available to look after the patient. In this situation, HP 12 considered that the patient needed to be referred to a welfare and counselling service

But some of the families, they said that if they take the patient home, who will look after him/her? ... that was the problem. There are many ... many of them, these types of problem are many ... Then, they refused to take the patient home. They refused to take home with all sort of excuses such as no one at home, patient will be looked after by the nurses in the hospital. That's the major problem ... sometimes we need to refer these types of cases to welfare service as we do not have counsellor, specific counsellor to counsel these types of family cases, have to refer to welfare service.

Patient attitudes related to self-awareness. Patients should be aware that they have had a stroke. It was felt that by being aware of their condition, patients would be more focused 
and responsible for taking care of themselves and reduce the risk factors for stroke. However, it was appreciated that this only applied to patients whose stroke did not affect their cognition.

Other important aspects of early rehabilitation practice post-stroke mentioned by a few health professionals included early exposure to various sensory stimulation and the reintegration or socialisation into the community. Families should always communicate with patients and provide some activities related to each sense such as identifying an object through its shape and size to provide sensory stimulation. "We cannot isolate stroke patients without exposure to stimulation such as touch, sound, hearing and vision" (HP 10). Some of the activities to promote socialisation included having patients engaged in hiking, watching soccer matches or other sport, listening to music and karoake.

\section{The benefits and risks of urutan tradisional}

The majority of health professionals identified about the benefits of urutan tradisional as an important rehabilitation activity. This finding is congruent with caregivers' and stroke patients' views. Health professionals believed that urutan tradisional would provide sensory stimulation, joint movement, improve sound production and as a form of exercise could produce various positive effects.

If they [patients] go for 'urutan tradisional', actually is good ... can recover ... it's good. Because when we use 'urutan tradisional' ... we introduce or we stimulate sensory. 'Urutan tradisional' is one aspect of treatments ... then ... some of the massager tries to exercise the joints. (HP 10)

Apart from exercises provided by the rehabilitation therapist, participant HP 12 also stressed the importance of urutan tradisional. "Then ... the exercise ... no ... don't forget ... go for 'urutan tradisional' ... exercise while practice conversation." According to participant HP 3 'urutan tradisional' was considered rehabilitation therapy. That 'urutan tradisional' is also important. It is an aspect of rehabilitation. If in the hospital, we have exercise, ROM [range of motion] and others, so 'urutan tradisional' also the same. 
Even though urutan tradisional was found to provide multiple benefits for stroke recovery, some health professionals had mixed opinions in relation to it. Two of the health professionals talked about the risks of this type of massage. For example it may cause shoulder dislocation due to aggressive or improper technique of massage. From their experience, stroke patients have had shoulder dislocation due to improper and aggressive technique of urutan tradisional.

But, the things that I am not really agreed with the urutan tradisional is because they [the massager] do the aggressive massage such as they do for shoulder. One of the patients had shoulder dislocation because of the traditional massager ... I do not know ... how s/he [the massager] did 'urutan tradisional' of the shoulder. s/he [the massager] said that ... s/he [the massager] found the nerve, and then ... s/he pulls it. (HP 2)

Health professionals were aware that some stroke patients only had urutan tradisional and that this resulted in contractures.

Some stroke patients came to us for physiotherapy three years after discharge from the hospital and developed contractures and flexion deformity. Before, s/he [patient] was admitted in the ward, but did not get physiotherapy. When I asked ... "What have you done within these three years? Have you visited the doctor?" S/he [patient] said that s/he [patient] just use 'urutan tradisional' (HP 2).

It was later revealed that this was partly due to the patient never being referred for rehabilitation therapy.

\section{Lack of knowledge among health professionals about the role of the rehabilitation therapist}

The lack of knowledge among the health professionals about the role of rehabilitation therapists leads to patients potentially not being referred to the rehabilitation service and negative attitudes towards the service. Patients who can get the most benefit from rehabilitation therapy should be referred to the rehabilitation service. However, some participants noted a lack of knowledge among some of the doctors on the role of 
rehabilitation therapists. Two of the health professionals talked about this lack of awareness possibly leading to a delay in a patient's recovery. Therefore some patients were discharged from hospital without follow-up rehabilitation therapy arranged. According to HP 1 it was surprising to find that some patients were left up to three years without any referral for rehabilitation therapy. In addition some patients did not realise the importance and existence of rehabilitation therapy.

Doctors, sometimes ... the doctors here, they do not know the role of occupational therapist (OT). Sometimes we miss patient ... then, patient came to the physician clinic ... we know that patient should be referred to OT [occupational therapy]; patient came with shoulder sublux, and with spastic hand. It is very hard to do the therapy, need longer time for rehabilitation.

This situation was thought to mostly happen during the transition period when some of the new doctors were just being oriented to medical discipline. HP 1 commented "We cannot just see patient like that. Doctors need to refer to us. The majority of the doctors do not know the role of occupational therapist." It was commented on by another participant that doctors "do not know the role of physiotherapist." (HP 2)

The doctor ... s/he does not know that stroke patients have an alternative treatment, rehabilitation therapy; s/he [doctor] did not refer them [patients]. Some of them [doctors] do not know that physiotherapist can do chest physiotherapy ... do not know that occupational therapist could make adaptation ... trained patient to be independent. He/she will not refer patient. (HP8)

The current policy was that doctors were the only health professionals responsible for making a referral to rehabilitation therapy and only the rehabilitation therapist could commence the therapy. HP 2 indicated that "of course, if the doctor did not refer [patients], we do not know about those patients. We [rehab therapists] are just waiting for the doctor to refer to us". Some stroke patients who were eligible for occupational therapists were not referred for occupational therapy. Some of the possible reasons might be a misunderstanding between the role of occupational therapist and 
physiotherapist. The public as well as other groups of health professionals often find similarities between the two professions.

\section{As HP 1 said}

Just recently doctors have been more aware of the role of the occupational therapist. Before, when I first started, "What's OT [occupational therapy]?" Whereas in the larger hospital, we [occupational therapist] are already there, but they do not know because we are synonymous with the physiotherapist ... they [people] get mixed up.

Participant HP 8 suggested that rehabilitation therapists should be more proactive and take the initiative to identify potential patients for rehabilitation therapy by participating in the ward round with the doctors. However, some health professionals themselves did not consider that rehabilitation therapy was important for stroke patients. Participant HP 1 related the suggestion made by rehabilitation staff that to refer patient to rehabilitation therapy was perceived as the rehabilitation staff having nothing to do. Participant HP 1 said

When see patient [stroke patient] who is unable to walk. She [other rehab staff] told the doctor, "Could you please refer this patient for rehab therapy?" Then the doctor replied, "Have you got anything else to do?" We feel sorry for patient. In relation to the provision of information, HP 1 commented on some of the medical officers who were not fulfilling their role to provide information related to the function of the rehabilitation therapist.

After discharge, patient stayed at home. There is no provision of information from the health professional that first saw her [patient]. In this particular context it is the doctor. The doctor should aware of our function. Shouldn't let patient discharged and was on wheel chair for more than one year. Then when she [patient] came here [rehabilitation unit], after two to three months she was able to walk, lift her hands and pick up things. From there we already know ... but there is lacking in the provision of information ... just give medicine, then discharge. 
When the BP is stable ... patient was discharge. How come she/he [doctor] did that?

\section{Educational needs of stroke patients, family caregivers and the public}

\section{(i) Educational needs on stroke, stroke care and rehabilitation}

The majority $(n=7)$ of health professionals talked about the educational needs of stroke patients and their caregivers in relation to stroke, stroke care and rehabilitation. Similar topics to those discussed by stroke patients and carers were raised. Information needs about stroke included the nature of stroke, stroke causes, risk factors for stroke, types of stroke, the effects of stroke, stroke treatment, and prevention of stroke and complications.

Education is very important, education about stroke itself, what types of limitations that the patient may have, why has the patient become like this? Why ... when she/he [patient] becomes angry ... suddenly s/he feeling sad. (HP8) Health professionals suggested that many people do not recognise the symptoms of stroke and therefore do not seek help immediately. They also described the need for the community to be educated on preventing stroke.

A lot of us in the community out there don't realise that they have a stroke until they are in hospital and the doctor tells them ... ha, you just had a stroke ... you know what it is? .... you're experiencing it now ... alright? (HP7)

Then s/he added

And then stroke preventions ... how to prevent a stroke ... cholesterol, blood pressure, diabetics, exercise ... that sort of thing ... and how do you prevent this ... preventable disease ... this life style disease that we can almost say ... that is preventable. How do you recognise the risk factors and control them so that you don't get a stroke?

Apart from information on stroke itself, HP 7 indicated that there is a need to provide a clear understanding and the importance of rehabilitation and nursing care required by stroke patients. This is particularly for discharged stroke patients who are still ill.

Understanding of rehabilitation and the nursing care required to care for very ill patients who have been discharged ... they may be stable but they still need a lot 
of care ... so that support system I believe still needs work ... but that support system is very costly.

What do you do after you get a stroke? Rehabilitation ... why is it important ... how can you get it? ... How long will it last for? ... What are the aims? .... What are the goals? .... Why must it be done?

According to participant HP 7, it is very important that caregivers are provided with education to improve their understanding on the rehabilitation and nursing care required post-stroke. Then caregivers would be able to help patients during the rehabilitation period.

The education needed on various areas of stroke care were also expressed by the majority of health professionals. These included tube feeding, hygiene, positioning, prevention of pressure sores, medication, and managing co-morbid conditions such as the early identification of hyper/hypoglycaemia. As some stroke patients may be discharged with tube feeding, two of the health professionals, HP 11 and HP 12, mentioned the need to educate family caregivers on this as the proper technique in giving tube feeding is important to prevent aspiration pneumonia.

If a patient is quite severe, in terms of feeding, s/he would not be able to swallow, need for tube feeding. That is very important to establish. That type of patient may get aspiration pneumonia. Patients will be discharged with tube feeding. So, we need to teach the families tube feeding and to change it fortnightly.

\section{(ii) Public awareness of stroke}

Public education is needed to promote stroke awareness. The public generally needs to be educated on the care of stroke patients at home, as expressed by HP 3. There is some misunderstanding among the public about the need for patients to seek immediate hospital admission following the onset of stroke. Participant HP 11 stressed the importance of investigating the type of stroke that patients have because different types of stroke will be given different treatment. 
This is [stroke] only rehabilitation, we should give early education. Usually patients who are admitted ... caused by sudden attack, this kind of patient needs to be admitted. The purpose is to do further investigation to identify the causes of stroke, whether caused by hemorrhage or blockage of the blood vessels. It is important for patients to be admitted because different types of stroke will be given different treatment.

It was also appreciated that the needs of stroke patients change over time and are different between individual patients. In contrast, HP 6 considered that physical recovery related to carrying out activities of daily living (ADL) are more important than other aspects of recovery.

\section{Support needs}

It was common knowledge amongst participants that home-based stroke patients (HBSP) needed long-term care and support post-discharge. All of the health professionals talked about the need for support from various sources such as family members, the community, the health service and support groups. Support needs varied and may comprise physical, emotional and psychosocial support.

\section{(i) Support needs from the families}

Participant HP 11 talked about the importance of support from family members for stroke patients to facilitate and optimise recovery. The focus is more on the care and social problems. Family support could influence patients' outcomes as it could help patients face social problems while at home. Following that, it is crucial that patients and families were given appropriate advice, information, education and referral prior to discharge.

Actually stroke is more on the care, patients' social problem. Usually treatments were given during acute stage, while patient in the ward, after that, patients were dependent on the families. How far is their [families'] understanding? If patients received good support from families, patients would survive longer. Because ... without good rehabilitation, patients would not survive longer. 
It was felt that the support from family members should commence while the patients were still in the hospital.

We ... as health professionals are unable to give intensive or 24 hour care. We need help/assistance from family members ... because stroke patients need care all the time. (HP 10)

This need for support and involvement of families in the care of patients while in the hospital is consistent with HP 9's view that 'family members should not assume that the nurses would do everything for their sick family members in the ward because we are lacking staff and we really need help from family members". Patients were also considered to need support from family to increase their motivation. Motivation can influence a patient's compliance with rehabilitation activities and treatment.

\section{(ii) Financial support need}

Participant HP 7 talked about the lack of financial support currently received by the majority of discharged stroke patients and their caregivers. Cost for basic things such as special food and tube feeding changes needed to be met.

In the homes ... I think once they are discharge except for a very small minority that manages to go through the neurological rehabilitation system in Hospital $K \ldots$... Hospital $Y \ldots$ a lot of them, the support is not there ... even financially the support is not there ... a lot of them are struggling ... the welfare system is not picking up a lot of cases that we're seeing. Families are left in the lurch ... no money to pay for special milk and special food and tube changes.

Another participant, HP 3 believed that for the quality of life at home, "patients should understand their illness and have the intention to recover". However, HP 3 agreed that patients and their caregivers also needed support to avoid being in a stressful life. "Caregivers should be financially stable. Also need support from other family members and key person in the community as a resource person if any problems arise. Caregivers shouldn't be left alone, they will get stressed". 


\section{(iii) Hospital and home-based support/resource/education centre}

For the benefit of patients, HP 8 recommended that hospitals should organise activities or programme for stroke patients.

We lack a support service ... as patients were among the elderly, once they discharge home ... got nothing to do ... So, if the hospital, or club [developed] for stroke patient, it means that, for example, every Wednesday ... or every alternate day, there are some club activities such as aerobics or cooking activities to reduce fat.

Home support service in the form of temporary relief from caring activities was suggested to help family caregivers. “This support service ... is very important for stroke patients" (HP 8). It may relieve tension among the caregivers because they would be able to go out on their own for a while. During this period, a patient may be left at home under the care of the home support service.

Home service ... means that they take care of the patient for a while. Nurse will be at home ... then the children ... man or woman go out for a while ... relieve tension, go out with friends. Then when they [family member] come back, burn out symptom will be relieved. (HP 8)

\section{Religious activities as an important rehabilitation therapy}

Religious activities such as prayer and reciting the Holy Qur'an were considered important rehabilitation therapies. The importance of support from religious people in the rehabilitation activities were raised by HP 8 and HP 9. They were recommended that religious people should be included in the stroke rehabilitation team to motivate patients and families. This is congruent with HP 12's view that as Muslims, family caregivers should accept the care-giving role as a test from Allah. Religious activities were missed out or forgotten by therapists because they were not referred to in the standard assessment. From her point of view, prayer is a "must do" activity. Apart from the need to fulfil the religious order as one of the five pillars in Islam it was also thought that prayer would promote and stimulate movement. This is partly because prayer involves a few continuous activities such as standing, sitting, bowing and bending. However, the number and type of activities would depend on an individual's abilities. It is common 
belief among Muslims that religious activities also promote healing psychological, emotional and spiritual well-being.

What is written is about the "spiritual needs" only. That's the interesting aspect, prayer ... is very important to us. Stroke patients...sometimes they can remember, some of them have problem to see 'Holy Qur'an'. Because they are only able to see one side, so in order to pray ... once patients are able [regain the ability] to pray, able to do something that is compulsory, fulfils the religious order, they [patients] will feel happy. (HP 8)

Prayer is also good for memory as one of the compulsory activities in prayer is to recall, memorise and recite some verses from Holy Qur'an

In term of prayer, firstly is for memory ... then for movement. The movements such as from sitting to standing up, it means that ... for patients who have a problem such as paralysis of one side of the body ... they have the alternative ... by providing a stool at their side, so they can stand up. (HP 8)

\section{Caregiving role as a test from Allah}

One of the health professional participants, HP 12 believes that the caregiving role is a test from Allah and not many people have that type of opportunity. As stated in the Holy Qur'an, every Muslim should hold the belief that any burden or suffering is a gift from Allah. The participant considered that children have to make a sacrifice for their stroke family member and need to learn how to care for the patient at home. HP 12 felt that the role of the nurse is to teach them (children or family member) this new role. Besides that, HP 1 suggested that family caregivers should take every effort to reduce the burden of caring. In Muslim belief and culture, children are not supposed to have a feeling that looking after their sick parent as a burden because every Muslim is responsible to care for their old parents particularly during illness.

\section{Health professionals' perception of stroke rehabilitation}

Another important finding is related to the health professionals' attitudes towards and perception of stroke rehabilitation. There is a lack of programmes available for stroke patients compared with other groups of patients, such as diabetic or cancer patients. The 
involvement in stroke rehabilitation was considered to be less profitable and of low prestige.

No one wants to be the Chairman for 'Stroke Patients' and conduct the activities, because this type of association/society is less profitable. It means that you will not get any profit, but you have to spend your own money. If you join a political party, you will get ... you understand? You will get profit everywhere like other society. (HP 10)

HP 11 agreed about the lack of education activities for patients and families and considered this might be because "it is not glamour. Stroke doctor is not standard."

Finally, a few participants identified barriers for follow-up visits at the hospital. Participant HP 6 expressed that some of the obstacles included transport problems, living far from the hospital, family caregivers being too busy or working and lacking commitment, and a patient's inability to mobilise. Some other contributing factors are limited parking space, and the unavailability of parking for disabled people at the hospital.

\section{Conclusion}

The findings demonstrated health professionals considered there were several important aspects of early stroke rehabilitation needed including prevention of complications, provision of early exposure to various stimulations and caregivers' awareness of their role in stroke recovery. With respect to educational needs, various areas of education on stroke and home management should be given to patients and their family caregivers.

The findings also highlighted some patients could benefit from rehabilitation therapy were not referred to a rehabilitation service. Delay or non-referral for rehabilitation therapy may cause serious complications and delay recovery. This is especially significant for patients who have mild to moderate stroke as these patients are believed to obtain the most benefit from rehabilitation therapy. The findings also demonstrated the need to educate other disciplines of health professionals on the role of rehabilitation therapy in stroke rehabilitation. 
In terms of urutan tradisional, these findings support those from the patients and caregivers. The role of urutan tradisional is very important and gives great benefit for stroke rehabilitation. However, patients and caregivers should be aware of the possible adverse effects of using improper techniques of urutan tradisional and should not depend on the urutan tradisional as their only means of rehabilitation. 


\section{Chapter 8: The educational needs identified in the interviews with stroke patients, caregivers and health professionals}

\section{Introduction}

The two previous chapters presented the separate findings from the interviews with the three groups of study participants: the stroke patients, family caregivers and health professionals. These findings provide some understanding and insight on the current practices of the provision of education for stroke patients and family caregivers as well as early rehabilitation practice post-stroke. The findings also illuminate that stroke patients and families/caregivers require educational information to ensure improved outcomes for patients.

This chapter presents the integrated analysis from the separate findings of the interviews with the patients, caregivers and health professionals. This analysis was done at the completion of Phase 1 of the research, and was used to inform the basis of the discussion in Phase 2. The main focus of the integrated analysis is to identify the educational needs of the patients and family caregivers post-stroke. The findings are presented under nine headings. These are education related to (i) information following a stroke (ii) managing co-morbid conditions (iii) urutan tradisional (iv) feeding and how to increase the swallowing mechanism (v) muscle strengthening, preventing contractures, and building strength (vi) prevention of pressure sores and hypostatic pneumonia (vii) performing prayer (viii) coping strategies and (ix) information on any resources or support available. These educational needs arose from what had been said directly by the participants on what is needed for patients and families following stroke.

\section{Educational needs following a stroke}

All three groups of participants expressed the need for information following a stroke, related to stroke care and the nature of stroke itself. Patients and their caregivers expressed a lack of understanding about stroke and wanted information on the nature of stroke and how and why it happens. They wanted information on why stroke occurs suddenly, without any forewarning signs and for no apparent reasons. Knowing the 
signs and symptoms of stroke from the beginning was found useful. Those who had never been diagnosed with hypertension felt that it was very unexpected that stroke could happen to them. They also raised the issue of how they could access information on stroke and whether it was widely available. Furthermore, patient and caregiver participants were keen to know how stroke could be prevented and the probability of having stroke recurrence.

Patients and caregivers also demonstrated an interest in information about how to speed recovery and the best medicine for people who have had stroke. In addition, the caregivers were concerned about ways to help their family member in terms of improving movement, feeding, prevention of complications and stroke recurrence and the provision of emotional and psychological support. Caregivers in particular found difficulty in dealing with patients' behaviour. The lack of understanding and knowledge of the possible impact of stroke on patients' behaviour caused more pressure on the caregivers. Caregivers also expressed their need for information on how to communicate with patients with a problematic behaviour. Caregivers required strategies to deal with patients who became more sensitive, were easily angry, and had a lack interest and low self-esteem.

The various issues on information needs raised by patients and caregivers were supported by the health professionals who admitted that there was an inadequate provision of information to patients and families. It is worth reiterating that the evidence from the earlier findings revealed that this is due to several reasons including no standardised education plan during an acute stage or prior to discharge, nurses' lack of time and whether nurses had the knowledge and interest to provide education. The amount and types of information given depend on the individual nurse and their interest. In addition, from the patients' and caregivers' view, the overall information by the health professionals was more focussed on general medical aspects. For example, this related to asking patients to take medication or take care of their blood pressure which were hardly understood by the patients, rather than discussing stroke itself. 
In relation to the recovery journey, patients and caregivers wanted to know whether a patient's condition had progressed as expected. The fact that the speed and status of recovery vary between individuals due to many reasons should be clearly communicated to patients and families.

Other important concerns by the participants were in relation to the educational needs related to the management of stroke patients at home. Caregivers were found willing to help their stroke family members with the caring activities at home. In general, they need information on many aspects of care such as basic exercise that can be carried out at home, personal care, care of patient with a urinary catheter, safety at home, communication strategies and early detection of hypo or hyperglycaemia. Information needs on the proper and easier techniques of transferring were also expressed by many caregiver participants who usually did it based on their own judgement.

Another important issue raised that needs to be taken into account is the availability of resources, particularly education resources in the community. To date, in Malaysia, there are some differences in terms of the allocation and availability of resources between different states and regions. There are some areas where stroke patients are able to access daily rehabilitation activities with some fees or minimal fees for service. In contrast, in other places, these types of services are limited or do not exist although patients might be able to afford the services. For example, daily rehabilitation therapy provided by the National Stroke Association of Malaysia (NASAM) is available only in a few states in Malaysia. This service is not available in Kelantan and even some health professionals were unaware of its existence. In addition, people might not use the rehabilitation services due to being unaware of their availability. Therefore people should be given information about locally available services and how to access to them.

Many aspects of the information needs on stroke care expressed by participants were related to the continuity of care at home. In conclusion, stroke patients and their caregivers need information on many areas related to post-stroke care along the recovery process. 


\section{Educational needs related to managing co-morbid conditions}

Hypertension, diabetes and heart disease are the risk factors for stroke (Bonita, 2004; Ong \& Raymond, 2002). Findings from this study show that there is a need for patients and their caregivers to know how to manage co-morbid condition as three of the five stroke participants had at least one co-morbid condition such as hypertension. Some patients did not have a clear understanding of the significance of blood pressure and need to be given information on the importance of blood pressure management to prevent stroke recurrence.

One patient participant expressed frustration with how she was treated by the health professionals who she considered were too focussed on monitoring her blood pressure and ignoring other aspects of care. The questions raised by the physicians during the follow-up visits were mainly on their co-morbid condition. Limited and ineffective information on the importance of blood pressure management caused dissatisfaction among patients. Besides that, the very brief statements from health professionals without further explanation such as "you should take care of your blood pressure" were found meaningless to some patients. Patients wanted to take care of the blood pressure but some did not know how to do it, and others did not really understand the importance of blood pressure management.

Therefore information and education on how the co-morbid condition could increase the likelihood of stroke recurrence and might aggravate a patient's condition should be clearly addressed. As revealed in this study this is particularly related to managing patients at home. Health professional participants strongly recommended that stroke patients and their caregivers should all be given comprehensive information and education on stroke risk factors, treatment and complications caused by stroke.

\section{Educational needs related to urutan tradisional}

Almost all the participants believe that urutan tradisional is an important intervention during early stroke rehabilitation to facilitate recovery. From the participants' point of 
view, urutan tradisional was found to produce multiple benefits such as improvement in voice, increased physical movement, lessened asymmetry of the mouth, provision of sensory stimulation and restoration of blood circulation as well as promotion of motivation for recovery. Patients also used urutan tradisional in conjunction with modern treatment and exercise provided by the rehabilitation therapist. This is congruent with the views from the health professionals who supported and encouraged patients to use urutan tradisional. From the findings, urutan tradisional was commenced as early as the day of the onset of stroke and the frequency of using it decreased over time. However, some health professionals were concerned about the adverse effect from improper techniques of urutan tradisional that can cause shoulder dislocation.

In relation to this, knowledge of the various types of urutan tradisional, the roles and the adverse effects of urutan tradisional seems to be very important for home-based stroke patients (HBSP) and their caregivers. In addition, they also need to be given information on how they can use urutan tradisional (intensively) and whether they can use it in conjunction with modern therapy during early rehabilitation period.

Urutan tradisional is as important as exercise provided by rehabilitation therapists. Therefore, from the participants' point of view, it should be integrated with the exercise provided by them. It also implies that people need to be educated on this area and ways to ensure the utilisation of proper massage techniques. The Malaysian healthcare system encourages evidenced-based practice.

\section{Educational needs related to feeding and increasing swallowing mechanism}

Interviews revealed that stroke patients and caregivers needed education related to feeding. This aspect of care was only expressed by the caregivers and health professionals. At the early stage post-stroke, the majority of stroke patients had problems with swallowing and therefore had a high risk of choking. This clearly indicates the need for education on prevention of choking that may led to aspiration. 
In addition, caregivers were concerned about the diet that could be given to patients. These concerns included what should be fed to the patients for every meal as well as the types of food they could eat. Caregivers also needed information about fluids, in terms of the amount needed per day, types of fluid, and how to modify fluid taken to prevent choking. Patients with dysphagia and caregivers were found in need of information on how to improve the swallowing mechanism. Patients were concerned about the support that they needed during feeding. This might be due to their having difficulty eating or drinking. Caregivers also expressed their need for education on these areas possibly due to their roles and responsibilities in providing care for the patients. The interviews with health professionals revealed another aspect of feeding, that some patients and their caregivers should be educated on tube feeding and how to change the tube. This is due to some stroke patients being discharged home with tube feeding. Moreover, education on the proper techniques of tube feeding would prevent them from getting aspiration pneumonia. This kind of information need was often expressed by participants when patients were discharged with tube feeding.

Education on prevention of aspiration pneumonia due is one of the important interventions at the early stage of rehabilitation. Aspiration may lead to significant consequences such as death. As some patients are discharged home with swallowing difficulties, caregivers need to educated on how to manage these at the very beginning. The possible topics of education are the types of food and drink needed by the individual patients, how to prepare food, and proper positioning during and after eating or drinking. In addition, some patients have the potential ability to prepare their own food with assistance and training. This group of patients and their caregivers can be provided with some cooking and food preparing training organised by the rehabilitation service. However, there were still issues raised that this service was not widely available and or accessible it. 


\section{Educational needs related to muscle strengthening, care of the joint and prevention of contractures}

The main goals of stroke rehabilitation are to promote optimum recovery and independence and prevent complications. There are three types of rehabilitation therapies: physical (physio) therapy, speech therapy and occupational therapy. Findings show that urutan tradisional was widely accepted and utilised by patients as a form of rehabilitation therapy following a stroke. This indicates that the benefits, risks and role in stroke rehabilitation need to be clearly communicated to the patients and caregivers. There are some implications for practice that patients need information about it. The rehabilitation service provided the three types of rehabilitation therapy. However, interviews revealed that some patients were found to be underutilising them due to a several reasons including being unaware of their existence, never being referred and being unable to access it.

Evidence shows that the improvement in physical abilities is closely related to improvement in psychological and emotional state. Physical disabilities can reduce patients' self-esteem and self image which may then lead to reduced or low motivation. This could have an adverse effect on rehabilitation and recovery. The majority of the stroke patients need some form of physical therapy to promote independence in their daily living.

Moreover, another severe complication of stroke which might not be fully understood is depression. Much was written in the literature that depression has a strong association with low motivation for rehabilitation activities. The majority of the caregivers and health professionals expressed that stroke patients were found lacking in interest and motivation for rehabilitation activities and patients were perceived as 'lazy'. Health professionals indicated that this low motivation might be caused by the side effects of drugs such as antihypertensives. This situation has some implications for the provision of education especially for caregivers on the possible causes of low motivation among stroke patients. This is important as the negative perception of caregivers towards stroke 
patients may affect patients' recovery. The caregivers and health professionals also need to be educated on early detection of depression.

Interviews with health professionals also revealed the importance of prevention of complications for stroke patients at home. This includes the prevention of stiffness/contracture of the joints and muscles and shoulder subluxation. Patients and caregivers should be educated on the proper and regular positioning and mobilising as well as exercise. There is also the question of how to do this safely.

\section{Educational needs related to prevention of pressure sores and aspiration/hypostatic pneumonia}

Educational needs on the prevention of pressures sores and aspiration/hypostatic pneumonia were only highlighted by the health professional participants. This is because those families and people with stroke involved in the study did not have experience of managing these complications. The other possible reasons are the current status of patient participants who were able to mobilise. The health professionals indicated the importance of families and patients taking care of the patients' skin, proper and regular turning and positioning to prevent pressure sore. Complications such as pneumonia and pressure sores can increase patients' mortality due to sepsis.

\section{Educational needs related to performing prayer}

The educational needs to performing prayer may reflect the context of the wider population within the study setting where the majority of population are Muslim. The current situation demonstrated the increasing awareness and interest towards religious practice among the population. All three groups of participants identified the need for the Muslim stroke patients to perform prayer as it is one of the five pillars in Islam and must be done five times a day. People are expected to abide to certain rules in performing prayer. This involves reciting specific versus of the Holy Quran, doing some specific physical movements such as sitting, standing and bowing, as well as taking ablution. However, there is some flexibility whereby prayer could be done according to individual abilities, and in particular for those who have altered health status. In Islam, people with altered health status are still required to pray unless they have an altered 
mental status. Interviews revealed that caregivers were concerned about how much and how far they should assist their stroke family members to pray if the patients were still wearing sanitary pads or diapers and their cognitive abilities were unaffected. The caregivers realised they had a responsibility to assist patients to perform prayer. When it came to those patients who had impaired mobility or were bedridden, the caregivers were also uncertain of their roles. Bedridden patients or patients with severe physical disability or incontinence were found very challenging to their family caregivers especially in taking ablution. Similarly with prayer, information on the right way to perform ablution is also required as it may depend on the patient's ability.

Some health professionals suggested that assessment of the patient's ability to pray should be done by the rehabilitation therapists. Indeed, they also recommended that the patient's assessment form which is based on western practice should be modified to include prayer as one of the basic physical assessment and rehabilitation activities as performing prayer involves some physical ability.

The information needs related to performing prayer were found more significant when patients were discharged home. This is the time where families were concentrating on providing care for patients with some residual effects of stroke.

\section{Educational needs related to coping strategies}

Evidence shows that the sudden onset of stroke and the devastating effects caused by stroke are one of the very challenging events in the patients' and caregivers' lives (Faircloth, Boylstein, Rittman, \& Gubrium, 2005; Kalra et al., 2004; Walling, 2005). Patients and their caregivers were struggling to cope with the sudden effects of stroke especially for those family caregivers who were new to the experience. Therefore they needed some mechanism to emotionally cope with the effects of stroke.

In this study, the majority of the participants indicated that patients and caregivers needed knowledge and information on coping strategies. Some participants used their own strategies to cope with performing prayer, solat hajat (hajat prayer), and reciting 
Holy Qur'an. It is clearly stated in the Holy Qur'an that reciting the verses from the Holy Qur'an and performing prayer would assist in the healing process as well as promote health. Health professionals also suggested that retraining of patients to recite Holy Qur'an could also be used as a part of the cognitive training because some patients had a problem with memory. It can also be considered as articulation practice due to the movement of the speech articulators.

In Muslim belief, anything good or bad that happens or suffering of disease and illness is a test or challenge from Allah. But they have to make all the efforts towards recovery, which is what was practised by the majority of patients and caregivers in this study. Some patients and caregivers talked about the impact of stroke a test from Allah. Some of the caregivers coped with the caregiving role by believing that it was also as a test from Allah. Other caregivers coped by assuming that they had to make some sacrifices for their stroke parents by looking after them. Therefore religious people were believed to be important in providing education related to coping strategies from the religious point of view. They could provide emotional, psychological and spiritual support for the patients and caregivers. This also could increase motivation and act as one of the coping strategies for the effects of stroke.

The other strategies recommended to cope with the impact of stroke relate to avoiding stress. Having stress was found to be one of the major factors that contribute to stroke recurrence. Therefore patient participants used various strategies to avoid stress such as socialisation, meeting with other stroke patients, sharing experience on strategies for recovery and by performing prayer, solat hajat and reciting the Holy Qur'an. Patients and families should be given information on these aspects to help them to cope with the impact of stroke.

\section{Information on any resources/support currently available}

This study demonstrated that in general caregiver and patient participants expressed their need for general information on what supports and resources were available for them in 
the community. This was even more significant for those who were caregivers for the first time.

Information on financial support to enable them to buy basic facilities such as diapers and nutritious food for patients were among the issues raised by the caregivers. Others were looking for any source of support for obtaining basic facilities for the stroke patients at home such as wheelchairs. Besides that, in general, patients were found among low income families. To participants, the role of the welfare service in relation to patients or disability benefits for stroke were not very clear.

Patients and caregivers also required information on stroke support groups and a stroke society available in their local community. The patients and caregivers found it very hard to meet other stroke patients except when meeting them at the hospital. However, this was not possible for some stroke patients who had a problem with transport. Some patients remained in their house and found it hard to socialise. Meeting other patients or other people in the community or within the hospital was found to be beneficial to stroke patients in terms of improvement of self-esteem, feeling better and the ability to share experiences and learn from each other.

Information needs regarding the types of services provided by the rehabilitation unit were raised by caregivers and patients. For example, a patient who believed that he would be able to drive again was uncertain whether the rehabilitation unit provided that type of service or where he could obtain such a service. Although this patient made regular visits to the rehabilitation unit, s/he indicated that she/he never asked for information and staff never provided any. This led to the patient being in a state of uncertainty.

\section{Conclusion}

The integrated analysis of the findings from the three groups of participants provided some insight and understanding on the need for development of education strategies for stroke patients and family caregivers at home. Generally, the whole findings indicated 
that the patients and their caregivers had been insufficiently informed along the recovery journey. Therefore a question arose as to how the information was provided and how the patients and caregivers would like to be informed. The findings also provided insight on the importance of timing for providing information because the needs of patients and families may change over time. Attention should be given to providing information tailored to individual needs. This was raised as part of the core issues of the priorities and processes for developing educational strategies for HBSP and their caregivers. These include information on stroke disease and home-care, related to spiritual and religious practice, on managing co-morbid conditions, coping strategies, prevention of complications and the practice of urutan traditional within the local context. The next chapter will focus on the findings from the Phase 2 of the study which mainly focused on the identification of priorities and processes for developing educational strategies based on the needs identified in Phase 1 of the present study. 


\section{Chapter 9: Findings of Phase 2}

\section{Introduction}

This chapter provides the findings of the analysis of Phase 2 which involved two focus groups of three health professionals each and two individual interviews. The details of the procedures and processes on how the data were collected and analysed were described in the methodology chapter. To reiterate, health professional participants were some of those interviewed in the Phase 1. The objective of the focus groups and interviews was to obtain participants' views in relation to the processes and priorities of developing and implementing educational strategies for home-based stroke patients (HBSP) and their family caregivers as identified in Phase 1 study. As summarised in the previous chapter, nine major themes of educational needs of HBSP and their caregivers emerged from Phase 1 of the study. The two focus groups are named using the terminology FG 1 and FG 2 and the two individual interviews Interviewee 1 and Interviewee 2. The findings are presented under six headings: current educational activities for patients, caregivers' priorities and education, public education priorities, strategies for change: the development of a multidisciplinary stroke team (MDST) as a strategy for change, evaluation method for educational programme or strategies/activities, and policy changes for the future.

\section{Current educational activities for stroke patients}

Participants reported that educational activities for stroke patients and their families were mostly delivered in an ad hoc manner. Some people got them, but what they got varied and some people did not get any education. What was delivered was limited, and there was a lack of communication related to the provision of information between health professionals. There was no standard or formal education programme provided in the acute setting, prior to discharge or in the out-patient clinic, and there was no established policy related to educational activities. If there were educational activities happening, these mostly related to the purpose of providing care for patients. For example, washing and tube feeding were linked with what individual health professionals did with 
individual patients at a particular time. With regard to the provision of information in the ward setting, the health professionals admitted that not much information given.

As you know, as usual, we give ... very little information ... we usually give [information] to the patient while we do something [giving nursing care] to patients, that's the nature. (FG 1)

The information given while the patients were in the ward was by direct communication, usually one-to-one with patients or families when needed. Group education or provisions of written material were not common practice throughout the ward and clinic. Following discharge some education was provided during the home visit by the rehabilitation therapist. However not everyone gets home visit. The purpose of home visits is oriented towards carrying out the activities related to rehabilitation therapy.

There was a group of multidisciplinary healthcare professionals but they did not seem to work as an integrated team and were not referred to as a team. Participants spoke of stroke patients who had potential for recovery but who were not being referred to the rehabilitation service for many years, when a referral might have greatly influenced their recovery. There was a lack of integration and co-ordination in providing information amongst the multidisciplinary team and between the in-patient and home setting. As explained in Chapter 3, literature shows that improvements in patients' functional activities have a strong association with improvement in patients' well-being, self-esteem and reintegration into the community.

As post-stroke care involved the multidisciplinary team, focus group participants raised the issues of who within the multidisciplinary team should provide education, and how a team could ensure that all necessary information had been given. Some suggested that "medical doctors" who were the first contact person with patients should organise and co-ordinate appropriate referral for educational activities.

\section{Caregivers' priorities and education}

The majority of participants touched on the importance of caregivers' awareness in relation to the needs of patients and of their own need. In terms of education, it was felt 
that the caregivers should be aware of what they are lacking, what they need and what they want to achieve. The basic knowledge in understanding stroke disease was considered very important for caregivers. Caregivers who are knowledgeable were perceived as more effective in fulfilling patients' information needs. The health professionals suggested that caregivers need to have a clear understanding of the importance of the physical therapy as part of rehabilitation activities. "Patient education is important so that families do not assume that physical activities are hard and would cause patients to suffer” (Interviewee 1).

According to Interviewees 1 and 2 the hospitals only provided supportive and basic care. Rehabilitation of stroke patients is not $100 \%$ at the hospital. Most of the rehabilitation takes place at home. (Interviewee 1)

Knowledge of the nature of stroke and stroke impact was also essential as a preparation for the caregiving role and for family caregivers to be able to adapt to life changes after stroke.

One of the difficult issues raised by participants was the need for information on how to deal with patients' behavioural changes after stroke. Interviewee 1 reflected on his experience of many years in stroke rehabilitation and how such changes would jeopardise the family and the marriage relationship.

Stroke ... in terms of behaviour is very challenging. Education on the behaviour problem is very important. For example: sensitive and excessive jealousy. The lack of understanding on the behaviour changes between spouses may lead to marriage breakdown. (Interviewee 1)

The impacts of stroke on patients' behaviour as well as sexual issues were identified as important aspects of education for stroke patients and their spouses. To date, sexual counselling is not provided by rehabilitation staff.

Caregivers' attitudes may affect patients' recovery. Family caregivers who are overly protective of their stroke family member were identified as a group who needed to be 
targeted for education. Health professionals were concerned about caregivers who were overly protective

My mum will be in pain, tired, hard for her. Of course, physical therapy will cause some tiredness. Stroke patients always want to sleep. They lack sensory stimulation; to them ... sleep is the most comfortable way of life. (Interviewee 1)

Apart from the prevention of stroke recurrence, other important aspects are managing comorbid conditions. The caregivers' awareness of the importance of managing co-morbid conditions at home, such as blood pressure or testing of blood sugar, needs to be addressed by health professionals. The lack of awareness could be blamed on health professionals.

Families should have awareness to manage patients' co-morbid conditions such as hypertension, for example by buying a BP set or blood test ... depends on our [families] priority, whether we [families] wants or not. People lack awareness because they lack information, they do not understand the nature of the disease. That's our [health professionals] fault. (Interviewee 1)

It is also important caregivers are aware that stroke patients have the right to a normal life. Patients should not be neglected. In summary, caregivers' education on positive perception of stroke is believed to be one of the important areas that needs to be given attention.

\section{Public education priorities}

The public need to know about stroke, stroke impact and prevention of complications. This knowledge was found important as a foundation towards people's understanding of post-stroke care and any matters related to stroke. Furthermore, Interviewee 1 stressed the importance of public education and awareness that stroke patients need on-going care and rehabilitation at home and should not be neglected.

People do not understand, especially people who are living in the village ... that a stroke patient could recover if they were provided with enough guidance and training. Especially for woman who are at the age of above 60 or 70 ... no rehabilitation [rehabilitation therapy] at all.....no one help them. (Interviewee 1) 
Another important issue raised by the participant was in regard to the public perception and opinion that the responsibilities of post-stroke care are those of the hospital and patients' family members.

\section{The development of a multidisciplinary stroke team (MDST) as a strategy for change}

The findings of the present study demonstrate the need for education in many areas related to stroke disease and stroke care for HBSP and their family caregivers. Whilst discussing the possible education projects, the main priority identified was a need for a change of focus towards rehabilitation and for the development of education resources for Malaysian HBSP and their family caregivers. For change to occur there was a need for infrastructure to be developed within the healthcare service in the region. Participants believed that the change needed a planned approach that involved Malaysian policymakers, healthcare services, non-governmental organisations (NGOs) as well as community contribution. Participants were very clear of the need for the involvement of healthcare practitioners in relation to developing education strategies. All participants demonstrated a strong desire and full support for this and considered the priority was for the development of a multidisciplinary stroke team (MDST) in the region.

The idea of developing a MDST is in line with the nature of post-stroke care which requires a multidisciplinary team to deliver effective rehabilitation and recovery. It was considered by all that a MDST needed to be developed in a coherent manner, and to work together as a team. The health professionals were really positive and believed that the development of a MDST in the region would become a foundation in developing education strategies to meet the educational needs of HBSP and their family caregivers. It was considered that such a team would be able to deliver a co-ordinated and integrated service that could reach all patients, and ensure patients were referred to appropriate disciplines prior to discharge.

Participants suggested that the development of the MDST needed to be made known to the public through road shows, exhibitions and mass media such as radio. These 
activities were important so that interested individuals in the community could contribute as members or give a donation. Some participants offered that poor public awareness of the benefits of a MDST was the reason for the lack of support from the community for this initiative. Furthermore, a participant also indicated that the "Ministry of Health should be aware of the importance of this type of programme. Because it is part of the treatment" (Interviewee 2). The importance of MDST as an initiator towards a successful rehabilitation and provision of education at home was expressed by FG 2: "success at home may depend on our team [MDST] in the hospital."

Therefore the current way health professionals work needed to be strengthened to ensure a successful implementation of education for patients and their family caregivers. Participants wished that all sorts of education could be provided to HBSP and their family caregivers in terms of theory and skills through the MDST. Therefore there was a need to mobilise group action while enhancing individual care. All participants agreed that with effective education by the MDST, the consequences of stroke and the rate of stroke recurrence could be reduced, complications minimised, quality of life maximised and optimum independence promoted. In general, participants considered the time frame needed to develop this team might be a few months to one year. There were five main issues related to the participants' views on what the MDST should look like: governance, team personnel, team engagement with patients, team priorities, and educational activities that could be undertaken.

\section{Governance}

There were different views in terms of the location of the MDST and how the team should be operated. There were two views in terms of where the stroke team should be located. The first view was that the MDST should be hospital-based and funded by the hospital but integrated with NGOs as well as voluntary community members. All public or community contributions would go through the team. The rationale for this view was to avoid inappropriate use by the public and to enable health professionals to monitor the activities conducted by the team. 
Also by having the MDST within the current system, it was assumed that better communication would occur between disciplines in terms of referral through the implementation of standard operation protocols.

Once we have this team, each discipline will assess what's ... needed. That's why it must be hospital-based. Indirectly, doctors will know and the referral system would become smooth. We need more education for doctors. We could announce to the doctors that we have a unit. Once stroke patients are admitted, make sure they will be referred to this unit. (Interviewee 2)

However, there was some concern expressed about the MDST being in the hospital. Developing and implementing such a programme would increase their workload. "The negative impact on staff ... very frequent home-visit would burden the staff ... because this programme ... would be on-going” (Interviewee 1). The current staffing arrangements were perceived as already tight. Participants considered that nurse-patient ratios would need to be reviewed otherwise the MDST would cause more burden to the nurses. Even having one additional stroke patient in the ward would increase their workload and was viewed as a potential barrier for them to be involved in providing education. However, restructuring and increased manpower would create more knowledge expertise. More funding was recommended to accommodate the proposed MDST.

Alternatively it was considered that the MDST should be separated from the hospital and operated solely as an NGO. The service should be operated at a specific location or centre as it was believed that hospitals were unable to provide this type of service.

These types of things [MDST], we cannot put the burden on the hospitals, no ... cannot, hospital is only to provide the basic. To train stroke patients in certain aspects such as self-care, vocational, social, must be done at home. To implement this sort of things, we need a committee ... It needs to be operated as $N G O$, if we want to depend on the government, the funding is enormous. (Interview 1) 
This participant further emphasised that the rationale for the NGO choice was "to improve community care on stroke ... need to develop a team. [We] cannot depend on the government. Hospitals cannot provide this service".

An issue that was raised about the location of the service was funding. Although both participants (Interviewee 1 and 2) had similar views that hospital should only provide supportive treatment and care, they had different views in terms of whether the MDST should be organised as an independent body or run by the government. According to Interviewee 2, "funding [for MDST] ... should be under [and organised by] the hospital". A benefit of the MDST being a centre outside of the hospital area or next to the hospital was that it would not interfere with the hospital routine.

However, those who believed that the MDST should be operated as an NGO considered it should still have a connection with the hospital. This was important so that health professionals such as rehabilitation therapists could provide their services on invitation or at a scheduled time based on demand. A few issues were identified as barriers for the development of the MDST as an NGO. Despite the suggestion that the MDST could be operated solely as an NGO, it was considered that this could be problematic to implement. NGOs need to raise their own funding to operate. Interviewee 1 expressed his frustration with the attitudes of the local people whom he perceived as lacking interest in voluntary or charity works.

It is hard ... because not many [people] to be volunteers ... due to the attitude ... communities' mentality who are not helpful, do not generate income [voluntary works].

People in the community were reportedly more interested in the activities that produced income or money such as involvement in political parties. Interviewee 1, who had experience in trying to initiate activities in an NGO related to neurological patients, considered it was hard to implement this type of activity. However, he also considered that the lack of public interest was partly due to a lack of understanding of the importance of this type of programme.

Local people were not interested to be involved in volunteer activities related to stroke patients. They are too individualistic. They really left the responsibilities 
to the government and family members. They are doing too much talking. (Interviewee 1)

Furthermore, health professionals were considered to be responsible for this lack of public understanding. Although Interviewee 1 strongly recommended that the MDST should be developed as an NGO, from his/her point of view, it was "a dream" to actually bring it into practice. It was felt by some that the majority of people in the community were busy earning money for themselves and were therefore unlikely to contribute to charitable activities. People were more focused on providing their own basic needs for living and were therefore unable to be involved in those activities. This led to public ignorance of the importance for patients to socialise and reintegrate into the community. In addition, local people were also perceived as lacking in interest in charity works compared with people in other states within Malaysia. Some even considered the lack of community involvement was specific to Kelantan.

\section{Team personnel}

All considered that the MDST should comprise health professionals who were experts or experienced in stroke care including nurses, medical practitioners, rehabilitation therapists (occupational therapist, physiotherapist, and speech therapist), nutritionists, psychologists and medical social workers. In addition it was considered that the MDST should include religious people, traditional massagers, people from drug companies, key members or interested individuals from the community, as well as stroke patients and caregivers. Representatives from NGOs and other key people from the community were considered important to contribute services and funding to run the MDST. It was stated that NGO representatives would know about obtaining funds and involving drug companies could increase people's access to supplements and milk for feeding. Those in FG 1 went as far as saying "invite them to be involved [company who produce milk]. They can give discount [on milk] for those who can less afford it". In addition, even though some members from the public did not have any health professional skills, their contributions were considered important because some aspects of services within poststroke care "do not need high skill jobs" (Interviewee 1). For example, relearning activities of daily living (ADL) during rehabilitation therapy such as cooking, shopping, 
and socialising in the community could be conducted by the volunteers from the community. However, participants commented that the greatest contribution to the team would be by rehabilitation therapists.

The religious people's contribution would be in providing education related to performing prayer and other coping strategies for patients and their caregivers.

How to perform prayer is important ... The techniques of performing prayer for patients ... actually prayer is good for spiritual, if patient are able to move, it is also for exercise .... (Interviewee 2)

Traditional massagers also should be included in the team. Although participants agreed about the various benefits of urutan tradisional, some participants were also concerned about potential adverse effects of this therapy option. Apart from being involved in the provision of education, traditional massagers should be included in the stroke team so that the team could monitor and guide their practice to prevent complications caused by improper techniques of massage.

In terms of 'urutan tradisional' ... not all of them are good or expert, some are nonsense. Especially ... at the neck, disturb the carotid. They massage both carotids ... it becomes worse. That sort of thing that they need to be aware of ... should include them [traditional massagers] in the team. (Interviewee 2)

The participant FG 1 talked about the experience with a patient who had a cervical fracture caused by an urutan tradisional. This was due to the patient already suffering from osteoporosis before having stroke. Having the massage aggravated the condition.

'Urutan tradisional' can improve blood circulation. Can improve joint function ... just like physio [physiotherapy]. Patient had osteoporosis ... had massage ... then got a cervical fracture. We need to know other illnesses that they [patients] have.

A general feeling of doubt towards the positive effects of urutan tradisional was expressed by some participants. However, in general, participants agreed that urutan tradisional provided benefits such as improving blood circulation. A few participants suggested that patients who really would benefit from urutan tradisional should be allowed to use it while still in the hospital. This might also provide some form of 
psychological support for patients and their family caregivers which in turn could positively influence the patients' recovery. By being in the team, traditional massagers would also gain knowledge about stroke. Participants suggested that stroke patients who used urutan tradisional successfully could provide education for other stroke patients by sharing their experience.

Any organisation or team needs someone to be in charge or act as a leader. With respect to this issue, participants generally agreed that medical people - the doctor - should be the leader or organiser for the MDST. This is due to the public recognition of the medical profession as having full authority and being highly respected in the community. In discussion of this, some participants expressed their feeling of dissatisfaction with the attitudes of some medical people. They were perceived as always giving orders and not always attending to patients' information needs.

Lastly, FG 1 commented on the need to establish and improve the provision of information by having stroke nurses within the ward setting. Besides other staff, a stroke nurse was considered as a key person in any education programme for stroke patients. This was also a strategy to overcome some of the barriers for providing education due to nurses' lack of time.

\section{Team engagement with patients}

It was acknowledged by the participants that the provision of education for patients and families should commence in the acute phase and should involve preparation for discharge and adaptation with the life changes after stroke. From FG 1's perspective, the family caregivers of stroke patients should learn to care for patients while they were still in the ward, with guidance and supervision from nurses. Appropriate assessment and referral should be carried out to identify patients' and families' educational needs. The focus of the MDST needed to be on providing information as well as rehabilitation therapy in the home setting. This was considered especially convenient for those patients unable to mobilise and with a problem with transport. Addressing needs at 
home would also enable individual patients to have their needs met in light of their personal living situation.

\section{Team priorities}

Participants therefore considered that the focus of the MDST should be on providing a comprehensive rehabilitation service as well as information for patients and families following stroke. Participants generally agreed that many educational activities could be implemented through MDST. Besides these the MDST could also make an important contribution to the development of standard and well-establish discharge plans for patients and families prior to discharge. In addition, FG 2 suggested that a stroke resource centre could be developed by the MDST. This resource centre could be used as a source of information and education for stroke patients, caregivers and the public as well as health professionals. It was considered that this centre should be properly organised and developed so that it could be used as a role model for other states in Malaysia. The centre could also be developed and available in each district of the state.

When discussing the possible impact of developing educational programmes for health professionals, participants generally agreed that these would improve their knowledge and experience. By having an MDST, health professionals would need to upgrade their knowledge and ensure their practice was current to provide relevant education. FG 1 admitted that they needed to update their knowledge because their basic knowledge was insufficient. From these participants' point of view, they also needed to be upskilled in various clinical skills related to stroke care. Although they acknowledged that their knowledge was deficient, participants did not voice the need for in-service training or courses to obtain this knowledge. One participant who supported stroke education being organised by NGOs acknowledged that involvement in this type of programme would improve health professionals' knowledge and experience.

\section{Educational activities that could be undertaken}

Working on the assumption that the proposed MDST would be developed, participants demonstrated their interest and keenness to be involved in the provision of education for 
stroke patients and their caregivers. All participants recognised the need for many areas of education for them should involve multidisciplinary team, commence in the acute setting and be delivered also at home. As expected, the detailed content of the stroke education programme would be tailored to patients' needs and individualised as the effects of stroke varied. Many factors would need to be considered before providing specific information such as "age, stroke severity, employment and individual needs" (FG 2). Activities needed to be on-going as the type of education or learning needs among the caregivers and patients might change over time. FG 2 stressed that patients and especially caregivers should also be asked about the types of education they needed and lacked, so that a referral could be made to the appropriate discipline.

Although the educational content of a programme was not really discussed by the participants, they had some views and suggestions on the methods for future educational activities. Methods included written material and group teaching in the ward setting as well as other methods to complement the current one-to-one education.

Remote area ... [provide] booklet, pamphlet, compact disc. Home-visit is also needed ... monthly ... initially ... everything okay ... just through telephone ... helpline ... periodic consultation. (Interviewee 2)

As there was a lack of written material in the ward setting, FG 1 considered that developing a pamphlet was essential to complement the current one-to-one information given.

The use of a pamphlet was not supported by Interviewee 1. For this participant a pamphlet was not as effective as a video presentation plus music, as well as direct discussions and demonstrations which were more interesting, stimulating and motivating and people could see the real activities involved.

Pamphlet ... people are not really keen to read. The impact is not very good. To me, the most effective is video presentation which contains a set of activities that need to be done by patient at home. Some of the examples of the activities such as how to feed and walk, climb stairs up and down, how to write, people can see. Reading is not very effective. (Interviewee 1) 
Interviewee 1's focus was on physical therapy and physical activities.

Besides this, participants were concerned that the educational strategies or methods that were most effective for the patients and their caregivers should be used. They hoped that information and education could be provided through home-visits, where direct discussion and demonstration could also be provided. In-home education was believed to be more contextually relevant. As transport was one of the barriers that was highlighted in the first phase of this study, in-home education could overcome this for some patients. These were assumed to be more relevant in terms of teaching "skills" as it was provided within their home environment in a real setting. However, it needed to be provided by a multidisciplinary team in a consistent and patient tailored-manner.

Another type of effective educational activity that was indicated by this participant was the use of "visual reality" (three dimensions format). This kind of activity was considered appropriate for providing education related to rehabilitation therapy. Visual reality can enable patients to visualise things more clearly and realistically. Organising seminars and exhibitions were also mentioned by the participants as a promotion for education and increasing awareness among the public, patients and their caregivers. The participants also suggested that activities should receive more attention from the mass media.

In addition the MDST could develop its own web-site and create an e-forum or elearning for HBSPs and their caregivers which they could access at any time. Participants thought that the web-site could be developed with consideration for local conditions. The preference was to use the Malay language as a medium of instruction and communication. The need to involve stroke patients in providing education was also expressed by a participant: "stroke patients themselves can be involved in teaching" (FG 1).

A 'help-line' system could be directly connected to the ward setting so that patients and their caregivers could be in contact directly to obtain any information. Another 
participant pointed out that this was more appropriate for patients who were in a stable condition.

Although the MDST was considered essential to improving education for public, patients and families, several obstacles were identified. There were expectations from the health professionals that family members would participate in the care of stroke patients while they were still in the ward, as preparation for discharge. These activities mainly involved clinical skills such as assisting with hygiene care, positioning or feeding. Participants talked about some of the barriers faced by health professionals to provide education for family caregivers in the ward. Some participants claimed that the majority of the caregivers were reluctant to be involved in providing nursing care for their family members in the ward or to be involved in the educational activities in the ward setting.

Some families are not co-operative. Once in the hospital [patient], families thought that the responsibilities of caring for stroke patients were left on the nurses. (FG 1)

Another constraint faced by health professionals was related to the inability to identify primary caregivers among the family members. Frequent changes of the caregivers were reported as causing problems in communication and affected the delivery of information. Health professionals talked about the importance of identifying who was going to be a primary caregiver so that there was a continuity of communication between nurses and caregivers.

In conclusion, the findings showed the need to restructure and increase manpower, create more knowledge expertise, have increased awareness among various disciplines, have more funding to accommodate the proposed MDST. This could contribute to a reduction in patients who were not being referred for rehabilitation therapy

\section{Evaluation method of educational programme or strategies/activities}

The health professional participants generally agreed that many educational activities for patients and caregivers could be conducted through the MDST. When discussing the evaluation of such a programme, the majority of participants suggested that it could be 
evaluated using questionnaires, obtaining feedback directly from the patients and caregivers and conducting other research. With respect to obtaining feedback on the effectiveness of the various methods of educational activities, a randomised controlled trial where "for example, one patient is given a pamphlet and the other a compact disc ... which one recovers faster" (Interviewee 1) was suggested.

\section{Policy changes for the future}

Another important theme that emerged from the discussion and interviews was identifying what was outside of the health system to benefit stroke patients. This discussion related to promoting the reintegration of stroke patients in the community. Participants discussed the need for policy changes in relation to stroke patients to facilitate re-employment, job opportunities and provide financial support. Participants suggested that financial support could also be obtained from NGOs and private organisations. Participants expressed that the government should think seriously about introducing policies that promoted re-employment based on stroke patients' abilities. This was more significant for those who were the primary source of income in a family. As Interviewee 2 said:

We need to assist the stroke patients to have a life like other normal people, for those who lost their job ... until they are able to do the work, work that is suitable for them .... It does not mean that stroke patients cannot contribute.

Returning to work was also a part of rehabilitation therapy as people were able to socialise and their self-esteem increased. Participants expressed that the current situation showed that insufficient attention was given to promoting re-employment for patients who had the potential to return to work.

Once the MDST was established, participants talked about the importance of developing a stroke prevention unit within the MDST centre to promote stroke awareness. This idea was very significant because it was the responsibility of the health professionals to provide education on secondary stroke prevention. It was evident in the literature that stroke patients who had recurring stroke were at greater risk for death and disability than those who experienced their first stroke. 


\section{Conclusion}

This chapter has presented the findings from the second phase of this study which involved focus groups and individual interviews with health professionals interviewed in Phase 1. Findings indicated the current practice of health professionals providing education for stroke patients and their caregivers was happening mainly in an ad hoc manner. There was no formal education programme provided for patients and families in the acute setting prior to discharge or in the home setting. To date, no established policy related to the provision of education for patients and their families. There was a strong desire by the participants to develop a MDST as an NGO or in the hospital within the current healthcare service as a foundation for establishing education strategies for HBSPs and family caregivers.

Suggestions were also made on other general strategies to benefit patients such as the promotion of reemployment, provision of financial support and creation of job opportunities for the HBSP. Findings also indicated that community contribution was greatly needed for effective and successful rehabilitation and to fulfil the educational needs of HBSPs and their caregivers. This was in line with what had been proposed in the theoretical framework that HBSPs and caregivers needed the involvement and participation of the community and healthcare service for effective rehabilitation.

The next chapter will focus on the discussions and implications of the findings of the whole study. 


\section{Chapter 10: Discussion and Implications}

\section{Introduction}

This chapter discusses the key findings of the study. This is based on the integration of the findings from the two phases of the study presented in Chapters 6, 7, 8 and 9. The discussion centres on the aims of the study to explore the educational needs of Malaysian HBSPs and their caregivers and to develop education strategies that will assist HBSPs and their caregivers to minimise the risks of complications of post-stroke and support the advancement of their quality of life. The discussion includes an examination of how the findings fit with current knowledge. This chapter is presented in three main sections. The first section commences with a reflection on the research. The second discusses the main findings in regard to the local context. Given the significant findings related to cultural and religious practice, the discussion elaborates on the meanings of such practices. The final section presents the implications, recommendations and conclusions of the research.

\section{Reflection on the research}

This research provides insight into some of the needs, specifically the educational needs, of patients and their families in Malaysia, in particular Kelantan. Due to the nature of the intended research, a qualitative approach with two phases of data collection was used. The decision to use a qualitative design compared with a quantitative approach gave the advantage of obtaining rich data by having direct conversations with participants. The participants had the opportunity to express their ideas and opinions using their own words (Kvale, 1996). Therefore the choice of a qualitative design using in-depth interviewing was able to fulfil the aims of this study. The findings presented the multiple perspectives of patients, family caregivers and health professionals. What was shared came from participants' first-hand experience and their roles and responsibilities related to stroke. The health professionals had experience from across the sector as they were the key people in stroke care. The caregiver and patient perspectives were individualised reflecting what had happened to people in their journey, their understanding and their observation about stroke care generally. 
The use of a two-stage approach was appropriate for my study because the intention of the second phase was to obtain health professionals' views on developing education strategies identified as a need in Phase 1. This involvement of the health professionals in designing the programme was important.

Significant experiences during the data collection stage for me as researcher were obtaining stroke participants, completing the transcription and conducting the focus groups. Selection of discharged stroke patients was based on the list of admissions to the medical wards which gave opportunities for any patients to be involved in the study and reduced bias in sampling. However, when contacted by telephone, many were unable to communicate, were very ill, had moved to another state, or were unable to be contacted with the telephone numbers provided. Some died after discharge. Sometimes the caregiver was unavailable. This process was quite frustrating and time-consuming and led to quite a stressful situation. Most of the time I had to contact them by telephone many times before being able to communicate with patients or family members. Some of the reasons included no answer to telephone calls; caregiver was unavailable and changes in the contact number. Recruitment difficulties when conducting research using qualitative design have been reported by others doing qualitative research (Gubrium \& Holstein, 2002).

Transcribing the interview data word by word in Malay was very time-consuming and I had some difficulties especially when the conversation was in the local Malay dialect. In the process of transcribing I listened and replayed the tapes many times. A benefit of this was that I was prompted to recall the actual conversations with participants and gained greater understanding of the participants' intentions. In addition, conducting the conversations using the participants' own language meant I was also able to provide the meaning as they intended (Minichiello et al., 2008). In preparing the English summaries, careful consideration was given to maintaining the meaning of the data. This translation process can be considered as part of the analysis of the data. This gave me an advantage as translating and summarising assisted the data analysis as I became very familiar with 
all the ideas in the interviews. It was thus easier to identify key ideas and issues within the context of the data.

There were some important experiences during the Phase 2 data collection process. Even though the earlier intention was to conduct two focus groups of the 11 hospital-based health professional participants, with five or six participants in each group, having some not available had its advantages for the other group members in the sense that they felt more free to engage in the conversation. This was due to the homogeneity of the group members with most members being of similar status and of equal professional standing in the organisation. As discussed in the research literature, the homogeneity of focus group members encourages conversation and expression of ideas during group discussion (Kitzinger, 1995). Being able to interview two of those who were interested but unavailable at the focus group times meant I could capture their views on prioritising needs and developing educational strategies for patients and families based on their many years' experience in stroke care and rehabilitation. Therefore focus groups and individual interviews worked well. Overall, the chosen study design and data collection tool were able to fulfil the aims of the study.

In the earlier part of the analysis, I chose to translate directly some Malay concepts into English words. Later I realised that the translation of those concepts did not show the exact meaning that participants intended. Some of the words or terminologies that referred to specific concepts were difficult to translate into English because of their specific meaning. This led to some problems and uncertainty for me as to whether to maintain the Malay words or to translate them into English. I chose to utilise the Malay words as maintaining them as said by participants is one of the criteria for ensuring integrity in qualitative research (Burns \& Grove, 2007). In making this decision I was aware that when it comes to reporting research findings, researchers need to use the language that is required by those who will need the research. To address the readers' need explanations about the meaning of Malay terms were provided. 
From the linguistic perspective, the language used by the community reflects its culture and each community has its own language and specific ways of describing daily activities (Myers, 2002). For example, in this study it was found that some words used by the participants referring to certain objects, activities and concepts may be understood and interpreted differently by other communities such as New Zealanders. Urutan tradisional (ngurut), menutup aurat, solat hajat, air zam-zam and air tawar are some of the words significant in this study. These words have specific meaning in the Malaysian socio-cultural and religious context and translation would not show the exact meaning. The word ngurut is widely used among Kelantanese and is the exact meaning for the urutan tradisional or traditional massage used by Kelantanese. The pronunciation of this word is more to Kelantanese dialect which is slightly different from dialects in other states in Malaysia. In addition, solat hajat which is a type of prayer done for a specific purpose is a common coping strategy practised by patients and their caregivers in Malaysia. This prayer is also part of fulfilling patients' and families' spiritual needs. Therefore I realised that it was important to maintain the word that was exactly said by people during the conversations. While performing the prayer, Muslims usually place bottled water in front of the praying area, and after the prayer is performed, the water is called air tawar (blessed water). Muslims later drink the water, in the belief that the water is able to cure their problems - physically or emotionally. Muslims place so much faith in the healing power of air tawar. In addition the function of air zam zam (holy water) is similar to air tawar in that stroke patients regularly drink air zam zam in the hope that it will cure their health problems. It is not known how well the water benefits the patients, but from the study it was shown that patients feel more confident that their problem is cured by drinking the water.

As this study employed a qualitative design there is no intention to generalise the findings to the wider Malaysian stroke patients and family caregivers. It is not representative of the population of HBSP and their family caregivers in Malaysia. However, this study has provided some useful insight on the various areas of education and information needs of Malaysian stroke patients and their caregivers at home. It also provides some important findings which have some significant implication for future 
study in the area of post-stroke care in Malaysia. This is in line with what has been reported by the Malaysia Ministry of Health $(\mathrm{MOH})$ in that research needs to be done to explore the situation of the Malaysian stroke patients in the community. In addition, this study also provides some important issues in relation to the current practice of health professionals in providing information and education for stroke patients.

\section{Discussion of the main findings}

The section is presented under thematic headings based on the findings of the study. The themes uncovered in this study are discussed and interpreted in light of the guiding theoretical perspective and relevant literature. Implications for practice, policy and further research are also present. The thematic headings are: The current educational activities happen in ad hoc manner; the educational needs of patients and families on stroke and associated issues, stroke care, home-management and recovery; other educational needs and rehabilitation practice that are culturally and religiously bound; developing educational strategies through integrated and co-ordinated MDST; and multiple educational methods and activities for the future.

\section{The current educational activities happen in ad hoc manner}

The findings revealed that the current educational activities for patients and families in the acute setting and post-discharge happen in an ad hoc manner. In general, the provision of information and education are lacking and did not meet many patients and families need. There were no formal educational activities or programme and no standard and established policy related to information and education provision. In addition, there was poor discharge planning as reported by health professionals. The lack of information and education provision led to patients and their families not being adequately prepared prior to discharge. Most patients and caregivers asked many questions that ideally should have been addressed before discharge. The knowledge on stroke and stroke care will certainly influence their rate of recovery. Boughton and Halliday (2009) and Ellis-Hill et al. (2009) have found that poor preparation prior to discharge affected patients' on-going recovery as well as the health of their spouse and family members. A shorter length of hospital stay might also contribute to patients and 
families being inadequately prepared for discharge (Knight et al. 2006). In this study four out of five patient participants were only hospitalised for two to four days.

The findings were similar to those reported in the literature whereby many patients and families were dissatisfied with the level and type of information and education provided by health professionals (Bakas et al., 2002; Brereton \& Nolan, 2000; Hanger et al., 1998; Hare et al., 2006; Jullamate et al., 2006; Oloffson et al., 2005; Rodgers et al., 1999; Wellwood et al., 1994). The study affirms that there are deficiencies in current practice in relation to the provision of information and education following stroke. For those who received information, many were dissatisfied with what they were given. Garrett and Cowdell (2005) suggest that the provision of information should be tailored to individual needs. Therefore the socio-economic, education level and wellness of patients should be considered when providing information to patients or their family caregivers. A lack of availability of education materials was also reported by health professionals.

Generally, the provision of education was unplanned and infrequent. Some educational activities were provided only when other therapy or nursing activities or procedures were being undertaken by health professionals. Few educational activities were conducted in the ward, and the provision of education was also lacking during follow-up visits where the emphasis was on obtaining information on the patient's medical condition. Most education was very brief and patients and families expressed the need for further explanation.

This study reveals a few factors that influence health education practice within the Malaysian healthcare system. These factors hinder health professionals or act as barriers to education being provided. These include inadequate time or busyness and heavy staff workload, insufficient knowledge on specific areas by some health professionals, the role of individual staff and family attitudes.

The current staffing seems very tight. To reiterate, the provision of education by health professionals relies mainly on the health professionals' knowledge, own interest and 
workload. There is yet no standardised education plan or policy across the hospital setting. The feeling of a lack of skills and knowledge among health professionals to provide education for patients and families in various areas of stroke and stroke care is a significant issue revealed in this study. This has implications for practice and for continuing education for health professionals. This situation has also been found in England (Bennet, 1996).

On the other hand, the family caregivers' attitudes were also a contributory factor. These attitudes included family's reluctance, ignorance and perceptions that post-stroke care is solely the responsibility of the hospital staff. Health professionals strongly believed that family caregivers should be involved in the caring activities from the acute setting. This was also part of a preparation for on-going care at home.

Anther important issue related to the lack of co-ordination and communication between the health professionals in relation to providing education. There is uncertainty among the health professionals as to who should provide information and to what extent. This reflects the need to improve co-ordination and communication between multidisciplinary health care professionals. This issue is compounded because other people who could be involved in the educational activities included traditional massagers, bomoh, other stroke patients and families, older people and others in the community.

\section{The educational needs of patients and families related to stroke care cover a wide range of topics}

The present study revealed the need for education on a range of topics on stroke, stroke care, home management and stroke recovery and rehabilitation. Topics included the nature of stroke, stroke impact, 'why' and 'how' stroke happens, stroke medication, prevention of stroke recurrence and faster recovery. In addition, the educational needs on stroke care and home management cover many aspects related to knowledge and skills in performing activities of daily living (ADL), managing co-morbid conditions, and preventing complications including pressure sores, choking, aspiration pneumonia 
and contracture. The detailed aspects of information and educational needs related to stroke and home management of stroke are summarised in Table 6.

Table 6: Summary of aspects of education and information needs of stroke patients and caregivers

\begin{tabular}{|c|c|}
\hline $\begin{array}{l}\text { About stroke and } \\
\text { stroke recovery }\end{array}$ & $\begin{array}{l}\text { - The nature of stroke, causes of stroke, how stroke occurs, } \\
\text { types of stroke, risk factor for stroke, the impact of stroke, } \\
\text { prevention of stroke recurrence, complication of stroke } \\
\text { - The nerves } \\
\text { - Stroke medication } \\
\text { - How to recover / faster recovery }\end{array}$ \\
\hline $\begin{array}{l}\text { Home } \\
\text { management } \\
\text { /post-stroke care }\end{array}$ & $\begin{array}{l}\text { - } \text { Positioning } \\
\text { - } \text { Prevention of complications (pressure sores, choking, } \\
\text { aspiration, hyper/ hypoglycaemia) } \\
\text { - } \text { Tube feeding, diet/nutrition/suitable food } \\
\text { - } \text { Basic exercises at home } \\
\text { - } \text { Hygiene } \\
\text { - } \text { Follow-up care } \\
\text { - } \text { House modification } \\
\text { - } \text { Return to driving } \\
\text { - } \text { Urutan tradisional (benefits \& risks) } \\
\text { - } \text { Care of the catheter bladder drainage (CBD) plus observation } \\
\text { - } \text { of urine } \\
\text { - } \text { Matient's condition that requires immediate referral to hospital } \\
\text { - Safety at home (safety items) } \\
\text { - Social interaction with patient } \\
\text { - } \text { Access to available resources: e.g. information on stroke, } \\
\text { community resources }\end{array}$ \\
\hline
\end{tabular}

A wide range of educational topics needed by stroke patients and their caregivers revealed in this study are consistent with what has been reported in other studies (e.g., Brereton \& Nolan, 2002; Eames et al., 2003; Hanger et al., 1998; Hanger \& Mulley, 1993; Hare et al., 2006; Wiles et al., 1998). The information needs on the nature of stroke and stroke care are widely discussed in the literature. Indeed, Anderson and Marlett (2004) contended that stroke patients and their families require more information at all stages of stroke. This is supported by other researchers who suggest that the types of information provided should be tailored to individual needs and that this need may 
change overtime. To reiterate, as revealed in Chapter 3, a wealth of evidence demonstrated the education needs of patients and families. However, limited information related to this issue was available in local literature. Therefore various methods and strategies for educating stroke patients and families are greatly needed. It seems that everybody who is involved in caring for stroke patients needs to take part in educating stroke patients and their caregivers.

Literature shows that education is very important for stroke patients and their caregivers. To summarise, some of the benefits of providing education include facilitating recovery through increased motivation and adaptation, less anxiety, greater adjustment to life changes after stroke, increased quality of life, increased self-satisfaction, reduced stroke recurrence, and improved patient compliance to treatment. In addition Langhorne and Rudd (2009) emphasise the importance of providing adequate information and training to caregivers while the patient is still in hospital. They recommend pre-discharge home visits because this approach reduces the stress of the transition from hospital to home.

The wide range of topics of education needed by patients and families is consistent with what has been suggested in the theoretical framework SCCM developed for this study. The SCCM suggests that patients and families need to be fully informed and prepared on the four main elements: skills, information, support and resources. As revealed in this study, patients and families should be taught a range of skills on ADL including positioning, feeding, exercise, and the prevention of complications including pressure sores, choking and aspiration, and contracture. The possible effects of stroke on patients' cognition require some form of relearning and cognitive training.

The SCCM was developed with a focus on the stroke patient. In comparison with other chronic diseases, stroke mostly affects the ability to perform ADL therefore the SCCM recognises the importance of the families' role in supporting stroke patients at home. Family are also considered as a major resource for stroke patients in other countries (Pound et al., 1999). In the context of Malaysia, families play a major role in supporting and managing patients at home. Even though most stroke patients' care is carried out at 
home, little is known about the involvement of primary healthcare services in this care. Once patients were discharged, it seems that the burden of care was shifted from the hospital to the families. The SCCM recognises that families and the home are the centre of care for most stroke patients and therefore resources should be provided into the home. Given the complexity of managing patients post-stroke, this study revealed the need for support for the caregivers. Mant et al. (2000) demonstrated that support services provided for patients and families post-stroke can significantly increase the number of social activities undertaken and improve a caregiver's quality of life. Their work provides an insight into the need for education to promote awareness among other family members and the public related to the various negative affects of stroke on family caregivers. Education is also needed create awareness that patients and family caregivers need support from various sources including other family members, healthcare service and community members.

However, the SCCM recognises that healthcare professionals are involved and support patients and families along the stroke trajectory. Their involvement, however, is to provide resources to patients and families. In this study, almost all the health professional participants agreed that the healthcare service needed to develop educational strategies for patients and families in preparing them for discharge and to facilitate adaptation to life changes after stroke.

Even though the need related to feeding did not clearly emerge from patient and caregiver participants, health professionals highly recommended education on this issue. Overlooked or unidentified dysphagia may lead to aspiration (Perry, 2001). On many occasions, families play an important role in preparing suitable food for patients with swallowing difficulty. This has great implications for practice as patients and families need skills and knowledge related to feeding.

The need for sexual counselling for stroke patients and their spouse is important as depression was found associated with sexual dysfunction among patients following stroke. Causes for erectile dysfunction may include medications such as beta blockers, 
clonidine, sertraline or narcotics. Other causes related to sexual dysfunction include persistent incontinence of bladder and bowel, prostate problems, hypertension and diabetes (Conroy et al., 2009).

\section{Other educational needs that are culturally and religiously bound}

Most of the educational needs of patients and families revealed in this study were similar to those in the literature. However, the study also brought into focus the educational needs of patients and families that were culturally and religiously bound. As described in the findings, there were four main aspects of early rehabilitation practice post-stroke that were related to Malaysian cultural and religious practice. These were urutan tradisional, performing prayer, reciting the Holy Qur'an, and other activities (solat hajat, air tawar and air zam-zam). These activities were widely practised by patients and their caregivers along the stroke trajectory for several purposes including fulfilling their religious obligations, facilitating recovery and healing, promoting well-being, as a coping mechanism and assisting with the healing process. These practices have not been reported in the literature and the study revealed that patients and families were in need of education related to these cultural and religious practices.

\section{Educational needs related to 'urutan tradisional'}

The study revealed that urutan tradisional was an important early rehabilitation practice post-stroke. As mentioned in the introductory chapter, urutan tradisional is a form of traditional healthcare practised and well-known among Malaysians. Many communities throughout the world have their own technique of massage whether in the form of modern massage or based on their cultural practice. The popularity of urutan tradisional among Malaysian stroke patients might be due to the fact that it has been accepted from generation to generation as one of the ways to treat paresis or paralysis. Lately, traditional/complementary medicine $(\mathrm{T} / \mathrm{CM})$ has been given attention and accepted as part of healthcare practices and is integrated into modern treatment.

Urutan tradisional is used by some stroke patients extensively during the early stage of stroke rehabilitation, even as early as at the onset of stroke. A possible reason for its use 
is the lack of a comprehensive stroke service or in-patient rehabilitation centre in the region. According to Langhorne and Rudd (2009) a comprehensive stroke service "covers most of the needs of patients with stroke, and ... is integrated in a way that provides a continuous patient journey - a seamless service" (p. 598). However, it is not known how effective urutan tradisional is for stroke recovery. This is an important finding within the local context as many Malaysian stroke patients depend on this type of intervention.

Patients are assumed to be more reliant on traditional therapies. However, it is not known whether this is one of the reasons for public scepticism towards hospital management of stroke in Malaysia (MOH, 2006). I am aware from personal experience that some stroke patients were never admitted to the hospital; rather they depended solely on the urutan tradisional. The use of urutan tradisional was also encouraged by health professionals although there were some different views among them in terms of the benefits of using it. This is due to the fact that although it gives a lot of benefit for stroke recovery, improper techniques in doing urutan tradisional may cause some adverse effects or aggravate a patient's condition such as causing shoulder sublux/dislocation. This raises an issue of the need for research in this area to promote safe practice of urutan tradisional. While patients and families need education on safe practice of urutan tradisional, it is argued that traditional massagers also need information on patients' existing health conditions that may contra-indicate using urutan tradisional. It is important to understand that traditional practice might differ within regions, within a country or among countries. Therefore some guidelines in relation to the use of traditional practice are needed to promote its proper and safe use. This finding supports the emphasis by the Malaysian $\mathrm{MOH}$ for research to be undertaken to clarify patients' cultural beliefs and rationale for their reliance on traditional therapies (NIHM, 2006).

Having known the importance of traditional practice for Malaysian HBSP, it is important to integrate this into the model of care for stroke patients and their family caregivers in Malaysia. In Wagner's Chronic Care Model, there is an emerging concept of cultural sensitivity in healthcare, whereby healthcare providers need to respond effectively to the 
diverse cultural needs of patients. However, it does not specifically address the importance of traditional healthcare practice post-stroke where the emphasis is on achieving optimum recovery and independence. Therefore the SCCM was designed to integrate the traditional healthcare practice and religious issues for stroke care as evidenced in this study. This is to support the increasing awareness and emphasis of its importance by the Malaysian healthcare system. To my knowledge, the practice of urutan tradisional for post-stroke rehabilitation is hardly found in the literature. The published studies related to traditional practice post-stroke were undertaken by Jullamate et al. (2006) and Kim and Yoon (1997). Jullamate and colleague's study aimed to explore the informal rehabilitation activities performed by Thai family caregivers for stroke patients at home. The findings demonstrated that massage and exercise were the two main rehabilitation activities performed by family caregivers for their stroke relatives. Kim and Yoon explored the use of traditional medicine such as herbal medicine for stroke care in Korea. They revealed that a third of residents surveyed indicated that they would choose herbal medicine if they suffered a stroke.

This finding provides an important insight into the traditional healthcare practice surrounding post-stroke rehabilitation in Malaysia. It is in line with the increasing interest towards integrating T/CM into the Malaysian healthcare system. Evidence from this study demonstrated that traditional practice should be considered as complementary to modern practice. Even though patients and families did not verbalise the need for education on urutan tradisional, the possible negative effects of urutan tradisional indicate this is necessary. Therefore it can be concluded that patients and families need information on the possible benefits and risks and contra-indications related to urutan tradisional.

\section{Educational needs on performing prayer for stroke patients with some residual effects} of stroke

An educational need related to performing prayer for the stroke patients is another important finding within this study as well as for the Muslim community globally. The Holy Qur'an provides clear guidelines on prayer performance for those who are sick. 
The situation within the study setting shows that there were some guidelines for the sick people to perform prayer presented in the form of pamphlets provided by the hospital religious unit. However, some issues and questions arose relating to performing prayer specifically for those stroke patients who had some form of physical disability such as hemiplegia or hemiparesis or who had incontinence.

The pattern of the prayer performed by Muslims is similar all over the world. This is governed by rules regarding some physical activities including standing, bowing, prostration and sitting. Due to the unique nature of the disabilities caused by stroke and shorter hospital stay, on discharge patients and their caregivers had inadequate knowledge in performing prayer with some physical disabilities. It seems that stroke patients and their caregivers need clear guidelines and education related to ways of performing prayer for "disabled stroke patients".

A proper guideline based on what has been ordered in the Holy Qur'an is needed to resolve this issue. Otherwise it is likely to continue to be an issue because the literature shows that in line with advanced treatment and care for stroke more stroke patients will survive and be discharged with some disability into the community. These caregivers are the core persons who will be involved in assisting the patients to perform prayer. With certain level of functional disability, some patients may require constant help from their family caregivers to perform prayer. This is not only to fulfil the patients' responsibility towards religious obligation but also the responsibility of the family caregivers to help patients to carry out this task.

Prayer must be performed five times a day as one of the five main obligations in Islam. For Muslim patients with some disabilities, performing the prayers is considered as a challenging task although they are allowed to do it only to the extent of their ability. This is due to patients needing to fulfil certain rules or procedures before and during prayer. Some of these rules or procedures include taking ablution before prayer and menutup aurat during prayer. Menutup aurat means covering the whole body except the face and two palms for women and from the belly button down to knees for men. 
According to the religion, a husband and wife are not allowed to have skin contact starting from the time when ablution is performed until the prayer is completed. Therefore the spouse of a stroke patient needs to be very careful while assisting their partner in performing the prayer. Sometimes, assistance or help from children are greatly needed to assist with ablution. Taking ablution and performing prayer is not an easy task for those who have hemiparesis or hemiplegia.

The educational activities related to performing prayer and the assessment of the ability to pray is an important activity for the Muslim patients and their caregivers. However, there are some differences in opinions among health professionals in terms of who should be responsible for providing education related to prayers in the hospital setting. Some of the possible reasons are due to the issues of health professionals' knowledge, increase workload as well as current hospital policy. Despite having a religious unit within the hospital, findings from this study shows that stroke patients and their caregivers are left uncertain in relation to education related to performing prayer.

The current assessment guidelines used by rehabilitation therapists for stroke patients which is based on the western practice needs to be modified to include the assessment of the patients' ability to pray. This is very relevant within the socio-cultural and religious background of the Malaysian population. Furthermore, this could also be generalised to all Muslims globally.

Stroke is an age-related disease and the majority of stroke patients are among the elderly (Butcher \& Frankish, 2006; Zwygart-Stauffacher et al., 2000). The ability to return to pre-stroke activities such as performing prayer or attending the mosque are meaningful activities for stroke patients. Some studies found that stroke patients' recovery is perceived in the form of the ability to return to pre-stroke activities which is inconsistent with the health professionals' goal that focuses more on physical recovery. Prayer is considered part of rehabilitation therapy. It involves mild-to-moderate psychological, physical and brain activity and provides benefits in terms of psychological, musculoskeletal and cerebral effects improving the muscular functions of geriatric, 
disabled and dementic patients (Reza et al., 2002). With regard to prayer, Muslims believe that prayer and reciting the Holy Qur'an accelerates the treatment of the sick. Furthermore, in addition to praying, a sick person may also need to visit an expert doctor, use medicines, and receive hospital treatment and some other form of special care.

To my knowledge, none of the literature mentions about the specific information needs for performing prayer among stroke patients and their caregivers. It can be assumed that the majority of the studies were not looking at the activities related to performing prayer. It might also because the participants involved in the studies were not Muslim. The issues related to religious and spiritual needs among the Malaysian stroke patients need to be explored further due to the multiracial and multi-faiths context of the Malaysian population.

In general, this study reveals the increasing awareness among health professionals and family caregivers of their responsibility to assist patients to fulfil their religious obligation in relation to prayer. This awareness is consistent with the current development among the wider population locally where awareness of religious practice is increasing.

\section{Cultural and religious practice}

Reciting the Holy Qur'an is one of the coping strategies practised by Muslim stroke patients and their caregivers. Some of the aims of the daily practice of reciting the Holy Qur'an are to seek blessing from Allah for better health for assistance in the healing process, as an emotional and psychological support, and to promote a peaceful mind. These various benefits of reciting the Holy Qur'an are stated in the Holy Qur'an itself. Indeed, certain versus in the Holy Qur'an are believed to have the specific purpose of assisting in the healing process. Some of the bomoh use certain verses from the Holy Qur'an to treat patients.

In addition to reciting the Holy Qur'an, a specific prayer called solat hajat (hajat prayer) is a common practice among Muslims. The word hajat means request. Solat hajat is an 
optional prayer performed when one requests refuge in Allah for the fulfilment of one's wishes. Following the tradition of the Prophet, Muslims perform the prayer usually during the latter part of the night and at other times, and when they need something - in addition to performing the obligatory prayers. One's hajat (request) can be made after or during the prayer is being performed. If people are not well enough to perform the prayer, they may ask other people to perform it on their behalf. Usually these requests are made to close relatives and religious leaders in the community. This type of prayer can be done alone or in a group. A group prayer is preferred because it symbolises that the group or community is requesting for the patient's wellbeing. Therefore it is a prayer done for fulfilment of wishes, such as to promote health, assist in the healing process, and to ameliorate psychological and emotional distress. Solat hajat is also used as a coping strategy and is one of the ways to sustain the marriage relationship among patients or their spouse caregivers who may have a problem related to sexual issues. Intimate or sexual relationship without marriage is forbidden for Muslims. This study reveals that wife caregivers often recite the Holy Qur'an and perform a solat hajat to overcome this emotional problem. The challenge to cope with sexual issues could be a major emotional burden to some, and sometimes might be greater than the physical burden. However, it seems that this sexual issue is not considered as a long-term or major problem to husbands of stroke wives. This is probably because Muslim men are legally allowed to remarry and have more than one wife in some situations.

The health professionals in this research also considered that reciting the Holy Qur'an could be utilised as part of rehabilitation therapy, specifically cognitive training as patients need to recall and memorise the correct way to pronounce verses. Similar to other languages, correct pronunciation is very important to avoid misinterpretation. And this is also important because patients need to recite certain verses in prayer as well. Furthermore, for patients with aphasia, reciting the Holy Qur'an is considered part of articulation training or exercise of the speech articulators.

From the religious point of view, good health or illness or anything good or bad that happens to us is given by Allah. In Islam, Muslims are encouraged to recite the Holy 
Qur'an every day whether in health or illness. Stroke patients are no exception. As they have the tendency to fall asleep for many hours, this study revealed that caregivers believe patients should fill their free time by reciting Holy Qur'an rather than doing nothing. For those who were unable to recite due to aphasia, some families provided the opportunity for patients to hear a compact disc player. It is stated in the Holy Qur'an that one can also obtain benefits by listening to the Holy Quran. Therefore it can be concluded that the way Malaysian family caregivers cope with the burden of the caregiving role might be different from what has been practised by people with other religious and cultural beliefs.

\section{Other traditional/cultural practice/exercise to promote stroke recovery}

The findings also demonstrate that a few activities related to the socio-cultural context have been practised by stroke patients and their caregivers to promote recovery. Such activities include walking on the grass covered by embun (dew) which is believed to speed up recovery. Some traditional massagers use this method to complement the urutan tradisional to treat paralysis. One of the traditional practices which is also integrated into the modern practice is covering a stroke patient's body with sand at the beach. This form of therapy is not only practised by some individual patients but also carried out by hospital staff. A group of health professionals from one of the Malaysian private hospitals used to take patients to the beach and covered their bodies with sand. This is one of the rehabilitation therapies to promote recovery for paralysis.

The current trend shows that eel (belut) is not only used as a food but also to promote healing from many illnesses. Some people take it to speed healing after male circumcision. With respect to stroke recovery, participants believed eating eel was good for the nerves as it was thought to help the nerve to recover and heal, and it also helped the person regain energy. This cultural practice could be researched to examine the possible benefits of using it. It is in line with the emphasis by the Malaysian health care system in encouraging research into $\mathrm{T} / \mathrm{CM}$ to ensure safe practice. 


\section{Educational strategies need to be coordinated and diverse}

One of the main aims of the present study was to develop education strategies for HBSP and their family caregivers. Suggestions for education strategies presented are based on the evidence from this study as well as from related literature. Two main education strategies emerged from the analyses: to develop an integrated and co-ordinated MDST and to implement multiple education activities for the future. This research revealed a readiness and interest in change among health professionals.

\section{Coordinated and integrated multidisciplinary stroke team (MDST)}

Findings from this study reveal that radical changes need to be implemented to fulfil the educational needs of stroke patients and family caregivers. The need for a change of focus to a planned approach towards rehabilitation and the use of education resources has implications for hospitals and for undergraduate and postgraduate education of health professionals. The development of the MDST within the current healthcare system and infrastructure was highly recommended. This approach was considered a priority and a foundation towards the effective provision of education for stroke patients and families. Given the current ad hoc process, a coordinated multidisciplinary team seems a logical step in providing comprehensive information, education and rehabilitation service for patients and families.

There were, however, different views in terms of how the MDST should be operated and where it should be located. Some thought the MDST should be solely operated as a nongovernment organisation (NGO) and others that it should be organised and funded by the hospital and coordinated in partnership with NGOs. As described in Chapter 2, a model

of post-stroke care as an NGO run by National Stroke Association of Malaysia (NASAM) is currently operating in a few states in Malaysia. However, this is not yet available within the study setting. To date, we do not know the effectiveness or the reach of the services provided by the NASAM. The reasons given for the MDST being solely an NGO and free from the hospital include budget constraints and staff workload. Conversely, some viewed that the MDST should operate solely under the hospital as this would result in the continuity of care from the acute setting to the community and enable 
monitoring of practice. This is important to consider as the rehabilitation of stroke patients should commence in the acute setting or as soon as a person's medical condition is stabilised and even on the day of the stroke (Langhorne \& Rudd, 2009; Pollack \& Disler, 2002). Besides that, patients should be comprehensively assessed by the MDST within 24-48 hours of the onset of stroke (Pollack \& Disler).

The finding of a lack of co-ordination between healthcare team members in managing post-stroke care where some potential patients were not referred for rehabilitation therapy is critical. Clearly some people who would benefit from rehabilitation are missing out. This has important implications for practice and indicates that co-ordination amongst healthcare team members in providing education is greatly needed. There were uncertainties among the health professionals about who was providing information to patients and who was responsible for it. This finding is consistent with a study undertaken by Hoffmann et al. (2007) in Australia. However, this issue should not be an issue as Edwards (2009) suggest that educational activities should be provided as ongoing activities by health professionals. Health professionals cannot wait for the MDST and must start talking and showing their interest in it. Health professionals, however, generally agreed that the MDST should comprise multidisciplinary healthcare professionals who are expert in stroke care as well as other members from the public including patients and their families.

Brashler (2006) believes that families should be included as part of the stroke team because stroke patients will not thrive in rehabilitation or in the community if they lose a supportive spouse. Once in the community, families are the main source of support for stroke patients. As proposed by the theoretical model SCCM for successful rehabilitation and recovery, patients and families are core in post-stroke care, are located in the community and are supported by the healthcare service and the community members.

The nature of post-stroke care involved multiple health practitioners due to the multiple and complex impacts of stroke on patients as well as their families. The need for development of the MDST for effective stroke rehabilitation to optimise recovery and 
minimise the consequences of stroke has also been suggested in the literature (Brashler, 2006; Langhorne \& Legg, 2003; Somerfield et al., 2006; Talbot et al., 2004; William, 2005).

Given the important role of the all health professionals in stroke care a hospital-based or community-based MDST which is integrated and co-ordinated with NGOs should be developed to strengthen the current system. Being an integrated service, people can access it quickly. This team should initially focus on co-ordination and communication amongst the health care professionals, patients and families. Whether it is hospital-based or community-based, the ultimate goal is to ensure that education is available and appropriate to the needs of stroke patients and their caregivers. Although details of education interventions did not clearly emerge in the study, health professional participants generally agreed that various educational interventions could be implemented through the MDST.

Another recommendation was that the hospital should develop a stroke resource centre within the MDST to have information for patients, caregivers, health professionals and the public. Furthermore, all members of the MDST should take responsibility in providing education tailored to the individual patients' and caregivers' needs. This is consistent with the literature (Garret \& Cowdell, 2005; O'Mahony, 1997). It seems that it is hard to fulfil patients' and families' educational needs without an integrated and coordinated MDST because the current system seems to work separately.

\section{The need for future multiple educational activities}

This study reveals the educational needs of stroke patients and their caregivers related to topics on stroke, stroke care and stroke services. Various methods of educational activities were recommended by the health professional participants. In general, they believed that the educational needs should be addressed using multiple media including video presentation, three dimension techniques, web-learning or e-forum with local language, seminars, one-to-one teaching, small groups and written material. Apart from that, the core ideas were that education should be tailored to individual needs, be on- 
going and delivered by individuals from across the health disciplines. This is consistent with the educational strategies used by Edwards (2009). He developed an evidencedbased comprehensive stroke education programme which involved the multidisciplinary team at a stroke rehabilitation unit at Ipswich Hospital. To ensure the effectiveness and success of the programme, a few strategies were implemented such as identifying the appropriate education and delivering it by the discipline that was involved in treatment and known to patients and families. Those education sessions were on-going and held weekly. This type of programme would give an opportunity for patients and families as well as community members to attend any education session based on individual needs. In planning the development of a programme health professionals need to be cognisant of recommendation by Hanger and Wilkinson (2001) that

Education has moved beyond telling people facts. Effective stroke education programmes are patient-centred and will require repetition, interaction and will include flexibility, opportunity to apply the new knowledge and skills and will use a variety of technique that are relevant to a person's abilities. (p. 114)

In addition, the chosen educational method should match the content of education material as well as be suitable for the patient's education level and socio-cultural and religious context. As the current information provision is more one-to-one, small group education was suggested to complement this.

To date, especially in the ward setting, information is presented verbally. A wealth of evidence suggests that written information should be provided to complement verbal information (Coulter \& Ellins, 2007; Eames et al., 2008; Johnson \& Sandford, 2005; Knight et al., 2006). As mentioned earlier, this is supported by Bhogal et al. (2003) systematic review of the literature found that written information alone is not an effective method to provide information to stroke patients and their caregivers. Therefore it is congruent with the literature that patients and their caregivers should be provided with written information about stroke and stroke care to reinforce and complement the information gleaned from direct contact with healthcare professionals. 
Developing various educational resources could also be considered a way to minimise the consequences of stroke. These in turn would involve policy changes, funding and staffing as well as the MDST which is integrated and co-ordinated with NGOs. The suggestion to develop a help-line for stroke patients is consistent with the findings from Lutz et al. (2007) that home telehealth could assist patients and their families throughout the recovery period in the home setting and within the community.

Apart from the main findings, this study also contributes to an understanding of how the role of families, in particular women, looking after a family member with a chronic illness. The present study has demonstrated the need for support among the patients and their family caregivers. Family caregivers need support from other family members in terms of caring for patients, a temporary relief from the caregiving role, for financial assistance and as a source of emotional support for patients. The importance of support from other family members in caring for patients has also been revealed in the literature. Robinson and Wright (1995) undertook a study to explore both the process and outcomes of a nursing intervention with a group of families who experienced difficulty managing a member's chronic condition. Their findings are consistent with the present study as one of the interventions that provided an opportunity for the family to be together for support and share the caregiving role provided some benefits for the families. These included bridging family members' sense of isolation through families engaging in a new and different conversation, enabling caregivers to request family support and health professionals being supportive in meaningful ways. Bringing family members together provides a great opportunity for them to discuss their difficulties in managing the patient at home. Given the common role of women as primary caregivers, healthcare services need to understand what women think is important and could make a difference from their perspective when nursing people at home.

The effects of stroke on the family are widely discussed in the literature. It is considered a family illness (Pierce, 2000; Smith et al., 2004; Sundin et al., 2000; ZwygartStauffacher et al., 2000). It is understand that families need a lot of support with their daily lives to care for someone with a chronic illness. Evidence from this study reveals 
stroke patients and their caregivers need financial support, re-employment, and support from other family members and the health service. Financial support in the form of a monthly allowance was seen as crucial in supporting stroke patients and their caregivers. This is particularly true where the stroke patient or caregivers are the source of income in the family.

\section{Implication for practice, policy and further research}

The main research findings discussed in this chapter bring into focus some important issues related to the educational needs of HBSPs and family caregivers in Malaysia. It has also provided some insight on the current status of education as well as recommendations for developing strategies based on the evidence. There are a number of key implications for practice, policy and future research that arise from these findings. In addition, the study is very relevant to the current situation in Malaysia due to the emphasis by the Malaysian government that stroke research is one of the priorities in the health sector. The findings demonstrated the interest for change towards improving poststroke care and education provided for patients. In general, there was willingness to engage in these issues among the health professionals. It highlighted the need for policy changes at national, local and organisational level around stroke rehabilitation.

The findings provide an insight to the need to establish a stroke education programme starting from the acute setting to the community, which is on-going and tailored to individual patients' and caregivers' needs. These types of programmes should also be open to anyone interested. As most of the on-going care for stroke patients happens in the community a policy related to providing education prior to discharge should be established. This includes the development of a discharge plan in which multidisciplinary health professionals participate. Discussion with families in identifying needs in preparation for discharge could be established.

Evidence from this study suggests that home visits should be established and participated in by the MDST. Within the study setting, home visits conducted by the rehabilitation therapist were already underway with some limitations in terms of staffing and budgeting 
and a lack of involvement from a multidisciplinary team. The benefits of the involvement of the MDST have been widely discussed in the literature as stroke recovery requires the involvement of multidisciplinary healthcare. Apart from the primary purpose related to rehabilitation therapy, home visits should also emphasise on the provision of education.

The findings show that health professionals need to be more aware of the importance of providing effective education for patients and families following stroke. Health professionals themselves need to have specific skills and knowledge in many aspects of stroke and stroke care, communication and co-ordination. Therefore a stroke education program for staff should be provided as part of a staff development programme. In addition to the MDST, there is a need to create a stroke nurse specialist role or stroke educator within the ward setting to oversee ward-based education.

The findings demonstrate the need for some policy changes related to financial aid/disability allowance, re-employment and job opportunity for stroke patients and their family caregivers. There is a concern regarding re-employment for stroke patients who have the potential to return to work. There is also a need to promote employer awareness of stroke. Employers should be more supportive, sensitive and empathetic towards their employees with stroke. Returning to work promotes reintegration of stroke patients into the community. This will obviously involve national policy changes. In addition there is a need for the establishment of a national policy related to driver training for stroke patients. This service could be part of the occupational therapy service. The ability to drive again could facilitate community reintegration, promote motivation, increase selfesteem and facilitate adaptation to life changes after stroke.

In line with the increasing focus on community care within the study setting, a policy to support stroke family carers should be developed. Community care is largely family care (Brereton \& Nolan, 2000), which needs to be supported through partnership with healthcare professionals. Brashler (2006) suggests that healthcare intervention should promote a family-centred approach which allows healthcare providers to acknowledge 
and respect family caregivers' needs without sacrificing patients' interest or authority. The findings highlighted the need for the establishment of a standardised referral system from the acute setting to the community.

This study also reflects the need for a policy within the rehabilitation service related to performing prayer. The occupational therapy service should include a cognitive and physical assessment of the ability to pray. Despite the fact that other multidisciplinary healthcare teams already work in the region such as in the surgical and medical areas, this study has shown that there is a need for a specific well co-ordinated and integrated MDST. The challenge for the future is to establish and develop the MDST within the hospital setting and expand the services to the community - co-ordinated and integrated with NGOs. Such a team would offset the unavailability of a stroke unit in the region and the lack of an in-patient rehabilitation centre in Malaysia and consequently have an impact on stroke patients' recovery. The team should incorporate traditional methods of healing and cultural beliefs and practices.

This study also reveals that "political will" could greatly influence the establishment of any stroke activities or services in the community. This is consistent with the statement by the $\mathrm{MOH}$ (2006) that the current situation shows a lack of a sustained political

commitment as the "focus of political interest, funding and research has been on cardiovascular diseases rather than stroke”.

NASAM is a model of stroke rehabilitation operated by an NGO. Available education resources provided by NASAM through an on-line service could also be used as a source education before the development of the MDST in Kelantan. As most of the written materials were provided in the English language, however it might not be appropriate for some patients.

\section{Further research}

This study raised a few questions for further research surrounding post-stroke care in Malaysia. The findings demonstrated the need to capture the true picture of the 
healthcare services received by patients and families following stroke. Research could be done through a retrospective review to examine the extent to which stroke patients were provided with a rehabilitation service. Such research would provide more comprehensive information on who has or has not been referred to a rehabilitation service. This kind of research will also provide an insight on the utilisation of rehabilitation or other services among stroke patients.

Research is needed on the effectiveness, benefits and roles of urutan tradisional and other traditional health practices in stroke rehabilitation. Further research is recommended for a comparative study on the use of traditional medicine or practice as well as the cultural practice of other ethnic populations in Malaysia. Comparative crosscultural research is recommended to explore the extent to which cultural practice and belief influences stroke recovery. As revealed in this study, patients and families utilise various forms of religious practice to cope with the effects of stroke. Research is needed to explore how families cope around religious practice.

Further qualitative and quantitative research with a larger sample size to represent the Malaysian stroke patients and their caregivers could be undertaken to explore further the resource and educational needs of HBSPs and their family caregivers. Research is also needed to explore early rehabilitation practice post-stroke among patients from various regions including the rural part of Malaysia.

Research is also needed to examine nurses' and other health professionals' readiness and competency to provide education for stroke patients and their family caregivers. Without any specialised knowledge and skills in stroke care, health professionals especially nurses are assumed to have only basic knowledge. Such research will provide an insight for the national healthcare policy-makers to develop a continuing education programme in stroke care. This will be the basis for the development of stroke nurse specialists within the current healthcare system. 
This research has revealed the need for change related to better meet the needs of HBSPs and family caregivers. The generosity of a group of stroke patients, family caregivers and health professionals to contribute to this research has resulted in an understanding of what resources are needed and what needs to change to improve the healthcare of stroke patients. The challenge now is for health professionals and organisations to work together and change the service to address the identified needs. 


\section{Appendices}

Appendix 1: Example of interview guide used for stroke patients and family caregivers

\section{SEMI-STRUCTURED INTERVIEW GUIDE FOR STROKE PATIENTS AND FAMILY MEMBERS}

\section{Background}

Thank-you for agreeing to share your experiences and ideas with me today. For my studies and research I want to produce some educational material for patients and families like yourselves who are home-based. My particular interest is to learn about the health needs of stroke patients (and their families or caregivers) as they manage in the home after having had a stroke. I have read a lot of material but that isn't the same as talking with patients and their families. I want to hear your stories and to learn about what you believe is important.

Today I want talk to you about what has happened to you and about how you are managing at home. I would like to hear about the education and the support that you have or are currently receiving. I also want to hear from you about other information or education or support that you would have found/ find helpful. Lastly, in relation to the development of a resource for stroke patients and families, I want to learn from you what information and education do you think should be in the booklet.

Section 1: To learn about what has happened in relation to the stroke, and to change in life circumstances, and managing at home

1. Before we begin with this can you please tell a little about yourself?

Probe:

- Story about life prior to the stoke, what happened with the onset and management of the stroke, what has happened to your life

- Any other medical condition

- The disabilities you have from the stroke

- Status of functional disabilities etc

- A little about social life, the cultural context of the family and community

- What you understand about the cause of your stroke

2. Can you tell me a little about your typical day and routine at home. What happens in your day? How do you manage? What are the particular struggles? What things do you find helpful?

How did you learn what to do or to manage? What have been the most influential helps in this learning?

What things have been the easiest to learn? What things have been hard to learn? Are there any areas that you feel that you could still do with some help in learning about?

3. Currently what are your goals for progress? Have you had support to establish or to achieve these goals? What information or support would most enable your continued progress during rehabilitation or during care at home? Have you received this support or information from anyone? 
Section 2: Interactions with health professionals and health services In this part of the interview I want to talk to you about the health professionals that have been involved in your care. I would like to talk to you about the interactions that you have had with health professionals, the learning and support that you have received from health professionals, and to learn about the follow up that you have been getting from the health services.

4. When you first had the stroke you would have lots of interactions with health professionals and health services. As you started to get ready for discharge what did people talk to you about? Did you find this helpful? Did you get the information that you needed? What were the best things that you learned?

5. Did you receive information in relation to how to do daily care at home? What information or training did you receive? Did you receive information about stroke as a disease, health event? Any health education about avoiding further strokes, or managing any underlying health problems? Etc

6. What information or education have health professionals provided about what should you do (be done) to assess your progress, and to assess improvement or deterioration of your condition?

7. Have you had or will you have follow up appointments with any health professionals? Tell me about these. What information has been provided to you and your families related to follow up care? Do you have a sense of what information that the health professionals will want to gain form you about your progress and how things are going? If you had any concerns, how would you raise these with the health professionals?

8. I am keen to learn from you how health professionals or the hospital currently manages this process? What are the follow-up arrangements/ how do they work? How do you feel about these? So far, are you able to comply with these? Or do you have any other alternative / choices? Do you get the most benefit from follow-up visits? What other information is needed to support you and your families or other patients to get the most out of these services and opportunities to meet and interact with health professionals post discharge?

9. In relation to all of the above how can you and your family members best be educated or supported in relation to these things?

Section 3: Interactions with people with strokes, other community groups, or community support.

The books tell me that mixing with others is important - that it can help to talk with other stroke patients and families, and caregivers; that it can help to mix with others, or 
to have guests at home. In this part of the interview I would like to talk with you about this aspect of your lives and care at home.

10. Tell me about you and your family - do you have friends or community members to talk to, or that come and visit with you?

11. Have you talked to other people with strokes or their families? Does this interest you? Do you see as being important or not so important? If it is important, how do you access this?

12. Are there other groups or people or community services that you find particularly helpful? or that you think are needed?

13. Do you think you and your family members should be able to share your experience with other stroke patients and their family members? How could you get support to make this happen? Have you had any suggestions? Do you think that a stroke association or community stroke support group would be helpful?

14. Did you know about available community resources that might have been available for you and your family in the home? How did you get this information? If we were to help families get this information more easily, what would we do?

Section 4: In relation to the development of a resource for stroke patients and families, I want to learn from you what information and education you think you and your families and other patients need.

15. In relation to you / your condition or other stroke patients, what do you think is most important in home-based stroke care -for stroke patients and the families? Name the pieces of information or skills that are/were particularly important you in the early phases of your home care. Please share with me your reasoning and insights.

16. What do you think about the resources that are currently available for stroke patients and their families/caregivers in the home?

17. What are your thoughts about how easy or hard it is to find out about or to access these resources? If we could improve access to the information or to the resources what would we do?

18. What additional educational resources are needed by stroke patients, their families and carers?

19. From your point of view - what general health information do patients and their families need to manage or rehabilitate post the stroke at home? Please share 
with me your reasoning and insights. What for you would have been the best method of delivering this information?

20. In relation to care at home, what care advice should be provided for patients and their families? Please share with me your reasoning and insights. What for you would have been the best method of delivering this information?

21. Drawing on the knowledge that you gained from your experience, what else can be done to support you and your families?

Thank you so much for giving me this very valuable information. 


\section{Appendix 2: Example of interview guide used for health professionals}

\section{SEMI-STRUCTURED INTERVIEW GUIDE FOR HEALTH PROFESSIONALS Background}

Thank-you for agreeing to share your expertise and knowledge in the interview today. As you know for my Ph.D. research, I am wanting to produce an education booklet for home-based stoke patients and their families / caregivers. During the early aspects of my research I have read various policy documents, best practice protocols, and explored the findings from research literature. Now I am at a stage where I want to hear from clinical experts and community groups with knowledge of stoke care what they believe is important.

Once I have gathered different forms of knowledge and information from this series of interviews - I am planning to sort through the materials and to plan the development of the education booklet. Once I have done this, I would also like ask you for some additional comment.

For today's interview I have four main interests:

- Firstly, to learn from you what you believe is important in the early rehabilitation of stroke patients in the community or in home-based settings;

- Secondly, to learn about your current practices in providing acute stroke care, health information and health education;

- Thirdly, what you believe the interfaces between health services, health practitioners, and stroke patients and their community based families need to be once they are discharged home;

- Fourthly, in relation to the development of a resource for patients and their families, I want to learn from you what information and education you think people need.

Before we begin with this can you please tell a little about yourself, your expertise and your roles in stroke care?

Probe:

Area of practice

Years of practice

Qualifications

Work with stroke care

Specific interests in stroke care

Section 1: To learn from you what you believe is important in the early rehabilitation of stroke patients in the community or in home-based settings

1. In relation to your roles and expertise, what do you think is important in homebased stroke care - for stroke patients and their families?

a. If you could name 3-4 things that you believe make a critical difference to the health and well being of stroke patients and their families in the early 
phases of their home care - what would these be? As you name these please share with me your reasoning and insights.

2. In general, what are the important specific skills and knowledge for quality of life in the home?

a. Turning now to the knowledge and skills that stroke patients and families/caregivers in the home need-From your point of view what are the crucial ones? How do you think stroke patients and their families/caregivers might get or achieve these?

Section 2: To learn about your current practices in providing acute stroke care, health information and health education

I know from our earlier conversation that you are involved as a health professional in the acute care and management of patients with strokes, I also know that it is important that there is some continuity between in-patient care and home-based care, so I am interested to learn from you about your current practices in providing acute stroke care, health information and health education in the acute care setting.

3. What are your current regimes? For health information - about stroke as a disease, health event? For health education - what to do? What information do you provide for stroke patients and caregivers related to follow up care? Etc

4. From your perspective as a health professional (name role), what are the key interventions that you offer stroke patients in the acute phase of their care? What are the key outcomes or measures or goals of progress that you set for the acute care phase? What in your view should be targeted for intervention or continued progress during rehabilitation or during care at home post-discharge? What should the family do (be done) to assess the improvement or deterioration of stroke patients condition in relation to these?

a. From your point of view - what are the critical factors in improvement /deterioration or mark on success that can be used or evaluated by stroke patients and caregivers? How can people best be educated or supported in relation to these?

Section 3: To learn what you believe the 'interfaces' between health services, health practitioners, and stroke patients and their community based families are and need to be once they are discharged home

Currently in this region, the provision of post acute stroke care is not easy to achieve. In our country/province we do not have acute stroke units. More commonly patients are discharged from hospital for rehabilitation or care in the home. There is a tension between the skills and knowledge needed by patients and families and care givers to make safe and effective care and recovery in the home, and the need for follow-up, advice, and further support from health services and health professionals.

5. I am keen to learn from you how you or your service currently manage this process? What are the follow-up arrangements/ how do they work? What bits 
do patients and families generally get right? Are there areas that this process struggles to get right? What else that can be done to support patients, families and their caregivers to get the most out of these services and opportunities to meet and interact with health professionals post discharge?

Section 4: In relation to the development of a resource for patients and their families, I want to learn from you what information and education you think people need Thank-you for all the information that you given me. There is a final area that I would like to ask you to about. The educational resource that I am keen to develop, will I think need to cover three different forms of education - firstly, there is a need for general health information and care advice, secondly, there is a need for a section that helps stroke patients, families and their care givers with information or with choices about where to go to find additional information, or how to get information in relation to people or services that they need, and thirdly, I think that there is probably some education needed in relation to the kinds of information that health professionals need or will want at follow up visits, or the kinds of questions that can be asked of health professionals and other services in relation to their concerns. In this part of the interview I want to hear your thoughts about information that you think would be important for each of these sections.

6. From your point of view - what general health information is needed by stroke patients and their families post acute care, post discharge and while at home? What is most crucial about this? Please share with me your reasoning and insights. And how is the best method to educate them?

a. In relation to home care for stroke patient, what are the crucial area of care or advice to provide for them?

7. If they are interested to learn about the experience of other stroke patients, how can stroke patients and their families/caregivers get access to these? Are there any associations or other stroke support groups that you know of, or would recommend? How can stroke patients and families get to find out about these and similar groups and activities?

8. What resources are currently available for stroke patients and their families/caregivers in the home? What are your thoughts about how well stroke patients and their families/caregivers currently do in relation to knowing about or accessing these resources? If we could improve access to the information or to the resources what would we do? What other additional resources are important for stroke patients and the families?

9. Thinking now about follow-up visits with health professionals. From your point of view, what kinds of information do you need from stroke patients and their families when they come for follow-up visit in order to evaluate their progress or improvement? How well do patients and their families currently do in being able 
to provide this? If we improve this area, and make this need for information more overt for patients and their families what would you recommend?

a. During follow-up sessions, what questions should stroke patients and families ask health professionals?

10. What else should be done to support the information needs of stroke patients and their families or care givers in the home? 


\section{Appendix 3: Example of interview guide used for NASAM member}

\section{SEMI-STRUCTURED INTERVIEW GUIDE FOR STROKE ORGANISATION}

\section{Background}

Thank-you for agreeing to share your expertise and knowledge in the interview today. As you know for my Ph.D. research, I want to produce an education booklet for homebased stoke patients and their families / caregivers. During the early aspects of my research I have read various policy documents, best practice protocols, and explored the findings from research literature. Now I want to hear from clinical experts and community groups with knowledge of the rehabilitation needs of stroke patients, their families and caregivers in the home, about what they believe is important.

Once I have gathered different forms of knowledge and information from this series of interviews - I am planning to sort through the materials and to plan the development of the education booklet. When I have done this, I would also like ask you for some additional comment.

For today's interview I have four main interests:

- Firstly, to learn about your organization's (eg. NASAM) contribution/activities related to health needs and support of stroke patients and their families or caregivers.

- Secondly, to learn from you what you generally believe is important in the early rehabilitation of stroke patients in the community or in home-based settings;

- Thirdly, to consider what we might need to do if we were aiming to improve or strengthen the interfaces between agencies such as yours and stroke patients and their community based families;

- Fourthly, in relation to the development of an educational resource for patients and their families, I want to learn from you and your organization (eg. NASAM) what information and education you think people need.

Before we begin with this can you please tell a little about yourself, your involvement and your role in this association? Probe:

What is your work with stroke association?

How long have you being working in type of role?

Tell me a little about your background and training, education etc

What are your personal interests in stroke care?

Section 1: To learn about NASAM contribution/activities related to stroke, health information and health education

I know from our earlier conversation that working for a stroke association you are involved in providing activities and support to stroke patients in the community, supporting health services, and lobbying for policy development. I am interested to learn from you these NASAM practices.

1. What are the key services that NASAM offers?

2. In relation to your activities... 
Do you provide health information? eg. - about stroke as a disease, health event? Do you provide health education - eg. classes, seminars? Do you provide information for stroke patients and caregivers related to daily life or living in their own home? etc.

Section 2: To learn from you what you believe is important in the early rehabilitation of stroke patients in the community or in home-based settings

3. Drawing on your expertise, knowledge and experience, what do you think is important in home-based stroke care - for stroke patients and their families?

a. If you could name 3-4 things that you believe make a critical difference to the health and well being of stroke patients and their families in the early phases of their home care - what would these be? As you name theseplease share with me your reasoning and insights.

4. In general, what are the important specific skills and knowledge for quality of life for stroke patients and family members in the home?

a. Turning now to the knowledge and skills that stroke patients and families/caregivers in the home need-From your point of view what are the crucial ones? How best can stroke patients and their families/caregivers get information or education or achieve these?

Section 3: To consider what information might be needed if we were aiming to strengthen the interfaces between agencies such as yours and stroke patients and their community based families.

(Ask only if this comes out as a key activity of the association) - It is very important that stroke patients, their families and caregivers know about and use the resources of organizations such as yours. In this part of the interview I would like to learn form you your thoughts about how well patients and their families currently know about or access services such as your own. Also I would like to learn form you how this knowledge can be improved.

5. In terms of patients and families learning about your organization - How do families learn to get involved with you?

What aspects of this process do patients and families generally get right? (knowing about you, contacting you for the right reasons, with the right requests etc). Are there areas in this process that patients struggle to get right? (What are the most common misunderstandings that families have in relation to your organization and services?) What are your thoughts about how to improve the level of knowledge that patients and families have about your services? What else that can be done to support patients, families and their caregivers to get the most out of these services and opportunities to meet and interact with health professionals post discharge? 
6. What do you think about the current support or the provision of health service for stroke patients and family caregivers in the home? For the future what areas need the most improvement?

Section 4: In terms of the development of an educational / information resource for stroke patients and their families, who are managing at home, I want to learn from you what information / education you think would be most helpful.

7. From your point of view - what general health information is needed by stroke patients and the families who are rehabilitating or being managed at home? What is crucial? Please share with me your reasoning and insights. What is the best method to educate them about this?

8. What community resources for stroke patients and families in the home are currently available? How well do families find out about these? If we could improve communication about these, what methods do you think would be most helpful?

9. If patients and families are interested to know about the experience of other stroke patients, how they can get access to these? Are there other associations or other stroke support groups that you know about or that you would recommend? How can stroke patients and families find about their activities?

10. Lastly, for stroke patients and their families who live in sub-urban or rural areas, what are the best methods of providing education and information for them? 


\begin{tabular}{l|l}
\hline TO & Che Rabiaah Mohamed \\
\hline COPY TO & Dr Katherine Nelson, Supervisor \\
\hline FROM & Dr Allison Kirkman, Convener, Human Ethics Committee \\
\hline
\end{tabular}

\begin{tabular}{l|l}
\hline DATE & September 12, 2007 \\
\hline PAGES & 1 \\
\hline
\end{tabular}

\begin{tabular}{l|l}
\hline SUBJECT & $\begin{array}{l}\text { Ethics Approval: No 124/2007, The educational needs of home- } \\
\text { based stroke patients and family caregivers in Malaysia. }\end{array}$ \\
\hline
\end{tabular}

Thank you for your application for ethical approval, which has now been considered by the Standing Committee of the Human Ethics Committee.

Your application has been approved and this approval continues until 31 June 2009. If your data collection is not completed by this date you should apply to the Human Ethics Committee for an extension to this approval.

Best wishes with the research.

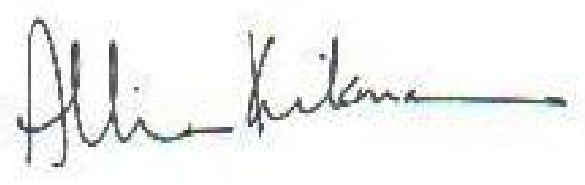

Allison Kirkman

Convener 


\title{
Appendix 5: Letter to Director of HUSM
}

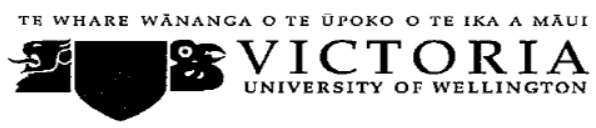

14 November 2007

\author{
Dr. Zaidun Kamari \\ Director \\ Hospital University Sains Malaysia (HUSM) \\ 16150 Kota Bharu \\ Kelantan \\ Malaysia
}

Dear Dr. Zaidun,

\section{Re: Permission to do a research at HUSM}

I am writing to request permission to carry out research at HUSM. I am a nursing lecturer at the School of Health Science, USM, Health Campus. Currently I am on study leave from the university to undertake my PhD in Nursing. I am completing the $\mathrm{PhD}$ at Victoria University of Wellington, New Zealand and am ready to undertake fieldwork.

The title of my research is: "The resource needs of home-based stroke patients and family caregivers in Malaysia". I would like permission to interview some HUSM staff and to access the stroke patients contact details. Various categories of health

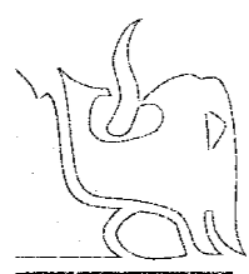
professionals from HUSM such as nurses, doctors, speech therapist, physiotherapist and occupational therapist will be approached for interview. The purpose of the interviews is to identify the practices involved in preparing stroke patients for discharge and to clarify what rehabilitation activities occur for patients in the community. The interviews will also ask the health professionals what additional educational resources they believe would be helpful to stroke patients and their families.

The other group of participants are stroke patients who have been discharged from HUSM and who currently live in their own home. Once I have obtained the contact details of stroke patients from the HUSM record office or ward admission book I will contact the stroke patients and their family to arrange for an interview at their home.

The Ethics approval to do the study has been granted by Victoria University of Wellington Human Ethics Committee. Please find enclosed ethics approval, an introductory letter from my supervisor and copies of the participant information sheet and consent form. 
I intend to come to Malaysia mid-January 2008 and stay for 3 months. Could you please write to me either at the address below or by e-mail confirming your approval for me to undertake this study. If you have any questions you can contact me or my supervisors by e-mail.

I am hoping you are in agreement as this research is important for the region. The findings will also be relevant for the hospital.

I look forward to hearing from you.

Thank you

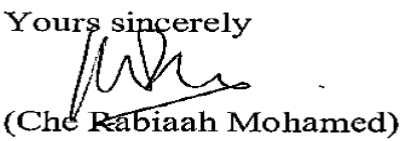

PhD Student (Nursing)

Graduate School of Nursing, Midwifery and Health

Victoria University of Wellington

PO Box 600,

Wellington, 6140,

New Zealand.

e-mail: aleef@hotmail.com

My supervisors:

- Dr. Katherine Nelson, Senior Lecturer, Graduate School of Nursing, Midwifery and Health, Victoria University of Wellington,

PO Box 600, Wellington, 6140, New Zealand.

Tel 006444636138 , fax 006444635442

e-mail: kathy.nelson@vuw.ac.nz

- Associate Professor Cheryle Moss, Rescarch Co-ordinator, Graduate School of Nursing, Midwifery and Health, Victoria University of Wellington, PO Box 600, Wellington, 6140, New Zealand. Tel 00644463 6141, fax 006444635442 e-mail: cheryle.moss@vuw.ac.nz 


\section{Appendix 6: Response from Director of HUSM}

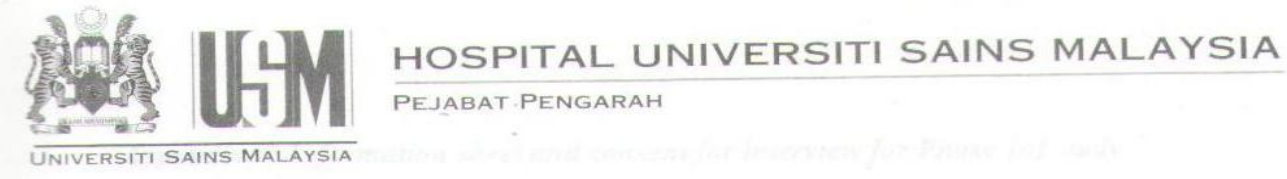

Ruj Kami: HUSM/11/020

Tarikh: $\quad 11$ November 2007

Che Rabiaah Mohamed

Graduate School of Nursing, Midwifery and Health

Victoria University of Wellington

P.O. Box 600

Wellington, 6140 NEW ZEALAND

Puan

\section{KEBENARAN MENJALANKAN PENYELIDIKAN DI} HOSPITAL UNIVERSITI SAINS MALAYSIA

Saya merujuk kepada surat puan bertariki 14 ivovențer 2007 mengenai perkara di atas.

Pengurusan Hospital USM membenarkan puan menjalankan penyelidikan bertajuk The Resource Needs of Home-based Stroke Patients and Family Caregivers in Malaysia' di Hospital USM tertakluk kepada syarat-syarat berikut:
i) Identiti pesakit dan keluarga hendaklah dirahsiakan;
ii) Gambar-gambar langsung (secara jelas) tidak boleh diambil;
iii) Sebarang bentuk laporan mengenai kajian ini tidak boleh diterbitkan tanpa kebenaran bertulis dari Pengarah Hospital ini;
iv) Data-data yang dikumpulkan hanya dibenarkan untuk perbincangan akademik sahaja; dan
v) Satu salinan kajian hendaklah dihantar kepada Pengarah Hospital USM.

Sila berhubung dengan Puan Hjh Sabariah Hj Jaafar, Ketua Penyelia Jururawat Hospital USM untuk urusan selanjutnya.

Sekian, terima kasih.

'EERKHIDMAT UNTUK NEGARA'

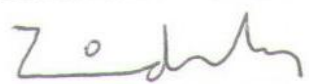

DR. ZAIDUN KAMARI

Pengarah

s.k.

Pn Hjh Sabariah Jaafar

Ketua Penyelia Jururawat, HUSM

Kampus Kesihatan, Jalan Raja Perempuan Zainab II. 16150 Kubang Kerian, Kelantan

Tel: 609-766 3000 / 609-766 3500; Fax:609-765 2198; E-mail: husm@kb.usm.my: Website: http:/husm/kb.usm.my 


\title{
Appendix 7: Information sheet and consent for interview for Phase 1of study
}

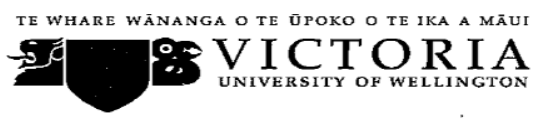

\author{
Information Sheet for a Study of the Educational Needs of Home-Based
} Stroke Patients and Family Caregivers in Malaysia

I am Che Rabiaah Mohamed a PhD student in Nursing at Victoria University of Wellington, New Zealand. I am undertaking a study to develop education strategies that will assist home-based stroke patients and their caregivers to minimize risk of complication post-stroke and support the advancement of their quality of life. This project has been reviewed and approved by the Victoria University of Wellington Human Ethics Committees and Hospital Universiti Sains Malaysia.

The first stage of the research involves interviews with health professionals (doctors, nurses, speech therapists and occupational therapists) currently working in Hospital Universiti Sains Malaysia (HUSM), key members of stroke associations, stroke patients, who have recently been discharged from hospital to home-based care and their caregivers. The purpose of the interviews with the health professionals and members of stroke associations is to identify educational gaps in the practices whereby patients are prepared for discharge from hospital and in the provisions made for their rehabilitation. The purpose of the interviews with stroke patients and their caregivers is to identify what rehabilitation is required and what patients and caregivers need in the way of information that would make rehabilitation possible.

Participation is voluntary. If you are willing to participate you will need to sign an informed consent form. You have the right to withdraw from the study prior to data analysis. There is no penalty for not participating or for withdrawing from participation. The interview will take place at a time and place suitable for both of us. I am able to

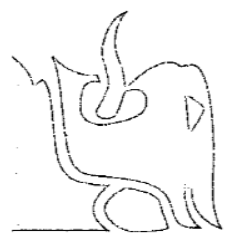
cover reasonable costs of expenses to attend the interview if required. The interview will take about 1 hour, and will be conducted by me. It will be recorded to ensure accuracy.

Once completed the interviews will be transcribed by me. The tapes will only be heard by me. Your responses will be treated in the strictest confidence. The tapes and interview transcripts will be locked in my personal cabinet. My supervisors will be shown sections of the transcripts without the names of the participants. The tapes and the transcriptions will be destroyed three years after the completion of the research.

Following the analysis of the interviews, decisions will be made by me about what education resource will be developed. The education resource will promote evidencebased practice, increasing stroke awareness among the public as well as healthcare professionals. It is intended that it will benefit people by minimising the risk of complication post-stroke and supporting the advancement of quality of life of stroke patients and caregivers. The resource should also assist the Malaysian healthcare policymakers in developing high quality health service based on research evidence.

GRADUATE SCHOOL OF NURSING, MIDWIFERY \& HEALTH

PO Box 600, Wellington, New Zealand

Phone +64-4-463 5363 Freephone 0800 108-005 Fax +64-4-4635442 Email nmh@vuw.ac.nz Website www.victoria.ac.nz/nmh 
All research findings will be reported in a way that respects the confidentiality of those involved. Care also will be taken in writing the thesis and the articles to ensure that individuals cannot be identified. In addition to education resource, the findings will be embodied in $\mathrm{PhD}$ thesis, which will stored in the libraries of Victoria University of Wellington, Universiti Sains Malaysia and in any other university libraries in Malaysia, for publication in academic journals and conferences. If you wish, a summary of the findings will be made available to you once the thesis has been completed.

If you would like to have further information or you have concern about the research you can contact:

- Che Rabiaah Mohamed

Graduate School of Nursing, Midwifery and Health, Victoria University of Wellington, PO Box 600, Wellington, New Zealand OR 40 A, Waipapa Road, Hataitai, Wellington, New Zealand OR

445, KM 3, Jalan Pengkalan Chepa, 15400

Kota Bharu, Kelantan, Malaysia.

Tel 006097481023 (Malaysia), 006443862188 (Wellington).

- Dr. Katherine Nelson, Senior Lecturer, Graduate School of Nursing, Midwifery and Health, Victoria University of Wellington, PO Box 600, Wellington, 6140, New Zealand.

Tel 00644463 6138, fax 006444635442

e-mail: kathy.nelson@vuw.ac.nz

- Associate Professor Cheryle Moss, Research Co-ordinator, Graduate School of Nursing, Midwifery and Health, Victoria University of Wellington, PO Box 600, Wellington, 6140, New Zealand. Tel 00644463 6141, fax 006444635442

e-mail: cheryle.moss@vuw.ac.nz 


\section{A Study of the Educational Needs of Home-Based Stroke Patients and Family Caregivers in Malaysia}

Researcher name: Che Rabiaah Mohamed

Informed Consent for Health Professionals, Stroke Patients and Family Caregivers

I have read the information sheet and have had the details of the study explained to me. My questions have been answered to my satisfaction, and I understand that I may ask further questions at any time.

I know that I may withdraw from the study prior to data analysis and I understand that my withdrawal will not affect my future health care or that of my family in any way.

I understand that this study has been approved by the Victoria University of Wellington Human Ethics Committees and Hospital University Sains Malaysia.

I agree to participate in this study and for the audio-taping of the interviews under the conditions set out in the Information Sheet.

I agree to review and comment on the education resources being developed

$$
\text { Yes }
$$

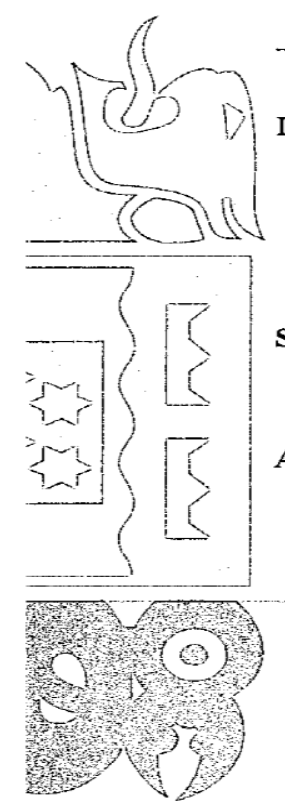

I would like a copy of the research finding.

Yes

No

Signature:

Date:

Address for draft educational resource and research findings:

GRADUATE SCHOOL OF NURSING, MIDWIFERY \& HEALTH

PO Box 600, Wellington, New Zealand

Phone +64-4-4635363 Freephone 0800 108-005 Fax +64-4-4635442 Email nmh@vuw.ac.nz Website www.victoria.ac.nz/nmh 


\section{Appendix 8: Letter and flyer requesting volunteer from NASAM to be interviewed}

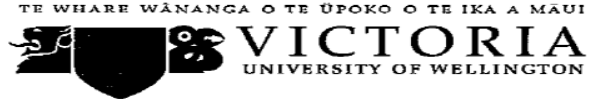

14 November 2007

The Director/Manager

NASAM

No. 12, Jalan $7 / 2$

46050 Petaling Jaya

Selangor Darul Ehsan

Malaysia

Dear Sir/Madam,

Re: Permission to do a research at NASAM Headquarters, Petaling Jaya.

I am writing to request permission to carry out part of my research at NASAM Headquarters, Petaling Jaya. I am a nursing lecturer at the School of Health Science, USM, Health Campus, Kelantan. Currently I am on study leave from the university to undertake my Ph.D. in Nursing. I am completing the Ph.D. at Victoria University of Wellington, New Zealand and am ready to undertake fieldwork.

The title of my research is: "The resource needs of home-based stroke patients and family caregivers in Malaysia". I would like permission to recruit and conduct research interviews with 1-2 relevant staff members at your centre. The purpose of the interviews is to learn about NASAM activities related to health needs of discharged stroke patients and their families or caregivers. The interviews will also ask staff for their opinions about the rehabilitation needs of stroke patients and their families, and their thoughts about what other general and educational resources might be helpful to them. The interviews will be conducted in Malay language.

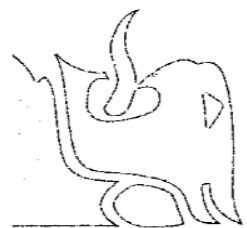

Similar interviews also will be conducted with other health professionals such as nurses, doctors, speech therapists, physiotherapists and occupational therapists who currently working at Hospital University Sains Malaysia (HUSM), Kelantan. I will also be recruiting participants who are stroke patients and their family caregivers (who have been discharged from HUSM, and who currently live in their own home) to talk with me about their needs.

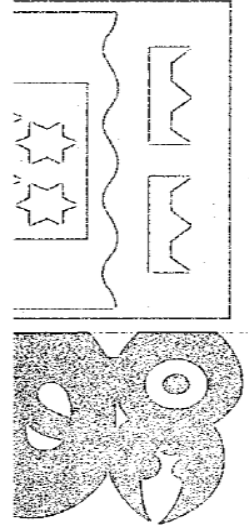

Ethical approval to do the study has been granted by Victoria University of Wellington Human Ethics Committee. Please find enclosed a copy of the ethics approval, an introductory letter from my supervisor, a flier about the research, and copies of the participant information sheet and consent form.

I intend to come to Malaysia mid-January 2008 and stay for 3 months. I would plan to visit your centre on one day (prearranged with you and the relevant staff) to conduct the

GRADUATE SCHOOL OF NURSING, MIDWIFERY \& HEALTH

PO Box 600 , Wellington, New Zealand

Phone +64-4-4635363 Freephone 0800 108-005 Fax +64-4-463 5442 Email nmh@vuw.ac.nz Website www.victoria.ac.nz/nmh 
interviews; each interview will take no longer than 1.5 hours. Could you please write to me (either at the address below or by return e-mail) to indicate your willingness to provide access to your organisation for the purpose of the interviews. Pending this approval could you please provide me with contact details of staff who may be willing to participate in the study, or draw the attached notice to the attention of your staff and ask interested staff to respond directly to me. I would welcome email contact with you. If you have any questions you can contact me or my supervisors by e-mail.

I am hoping you are in agreement as this research is important for the region. The findings will also be relevant for NASAM.

Thank you. I look forward to hearing from you.

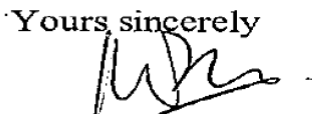

(Che Rabiaah Mohamed)

PhD Student (Nursing)

Graduate School of Nursing, Midwifery and Health

Victoria University of Wellington

PO Box 600,

Wellington, 6140,

New Zealand.

e-mail: aleef@hotmail.com

My supervisors:

- Dr. Katherine Nelson, Senior Lecturer, Graduate School of Nursing, Midwifery and Health, Victoria University of Wellington,

PO Box 600, Wellington, 6140, New Zealand.

Tel +64 44636138 , fax +644463 5442

e-mail: kathy.nelson@vuw.ac.nz

- Associate Professor Cheryle Moss, Research Co-ordinator, Graduate School of Nursing, Midwifery and Health, Victoria University of Wellington, PO Box 600, Wellington, 6140, New Zealand.

Tel +64 44636141 , fax +6444635442

e-mail: cheryle.moss@vuw.ac.nz 


\section{Appendix 9: Introductory letter from supervisor}

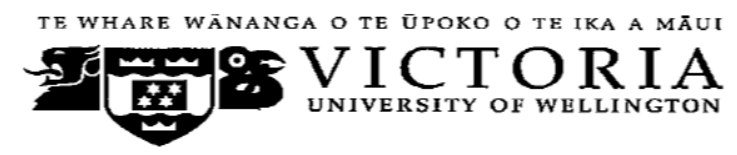

\section{Graduate School of Nursing, Midwifery and Health}

Letter of Introduction: Che Rabiaah Mohamed -Ph.D. Student

Referee: Associate Professor Cheryle Moss - Research Co-ordinator \& Supervisor

Dear Sir/Madam

I am writing to introduce Che Rabiaah Mohamed, to commend her research study to you, and to invite your support for organisational access for the purpose of data collection.

Rabiaah works as an academic at the School of Health Science, USM, Health Campus, Kelantan, where she teaches in nursing programs; she is currently on leave from this organisation to enable her to complete a Ph.D. Rabiaah is undertaking a Ph.D (full-time) at the Graduate School of Nursing, Midwifery \& Health. She is researching the educational needs Malaysian stroke patients and their carers who are being rehabilitated at home.

This is an important study as most of what is known internationally on this topic comes from western perspectives. The health care system, community infrastructure and societal values in Malaysia are very different to the contexts reported in the current published literature and research. Through-out much of Malaysia, patients with stroke are discharged home to the care of their families and lay care givers. The western traditions of managing post-stroke patient care in a clinical rehabilitation setting with expert nursing and allied health care are not the predominant process used throughout much of Malaysia. The burden of care most commonly is shifted to families and the lay community. This study investigates the educational needs of patients and families in these circumstances.

Rabiaah wants to interview a range of health professionals and members of community organisations who have expertise and experience in relation to the needs of stroke patients and their families for their opinions in relation to education needs and resources. In addition, Rabiaah intends to recruit and talk to stroke patients and their families, who are at home, about their needs. Data from these qualitative interviews will be analysed and Rabiaah will build a case and a plan for the further development of educational resources and material. The study design has been peer-reviewed at Victoria University of Wellington, and full ethical clearance has been granted by the University's Human Ethics Committee.

I am happy to be contacted by you or to provide additional material as needed, should this assist you. I can be contacted at Cheryle.Moss@vuw.ac.nz or on +64 44636141.

Thanking you in anticipation of your support

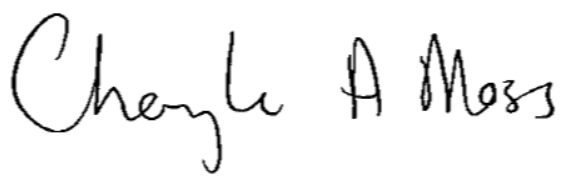

Associate Professor Cheryle Moss 


\section{Appendix 10: Information sheet and consent for Phase 2 of study}

VIOTORIA UNTVERSTTYY OF WELIIMGT'ON

Te Whare Wanarga o te Upokn ate ika a Maut

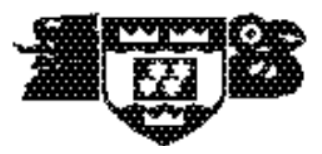

\section{Information Sheet for a Study of the Educational Needs of Home-Based Stroke Patients and Family Caregivers in Malaysia \\ Phase 2}

I am Che Rabiaah Mohamed, a PhD student in Nursing at Victoria University of Wellington, New Zealand. I am undertaking a study to develop education strategies that will assist home-based stroke patients and their caregivers to minimise risk of complication post-stroke and support the advancement of their quality of life. This project has been reviewed and approved by the Victoria University of Wellington and has been approved by Hospital University Sains Malaysia (HUSM) Director.

The study is in two phases, the first of which has been complete. The first phase involved interviews with health professionals working in HUSM, key members of stroke associations, and stroke patients who had recently been discharged from hospital to home-based care, and their family caregivers concerning views and perspectives on health education needs for people receiving home-based care following a stroke. This second phase involves only the health professionals who were interviewed.

In this second phase, two focus groups involving the health professionals will be held. The purpose of the focus groups is to discuss what was raised in the interviews from Phase 1 about health education needs of home-based stroke patients and their family caregivers and to identify priorities and processes for how these needs might be addressed.

Participation in this second phase is voluntary. If you are willing to participate you will need to sign another informed consent form. The focus groups will take place at time and date in the venue. The focus group will take 60-90 minutes, and will be conducted by me. It will be audio-recorded to ensure accuracy. Rules for the focus group are i) all participants will have an opportunity to share their views; ii) all views are respected; iii) consensus is not required about the priorities and processes decided by the group; and iv) an individual's views will not be discussed outside of the group.

Following the focus groups the results will be summarised by the researcher, the tapes will be listened to but not fully transcribed. The tapes and notes will be locked in a filing cabinet. My supervisor will be given copies of the notes. The tapes and notes will be destroyed three years after the completion of the research. 
All research findings will be reported in a way that respects the confidentiality of those involved. Care also will be taken in writing the thesis and the articles to ensure that individuals cannot be identified. In addition to the development of at least education resource, the findings will be embodied in $\mathrm{PhD}$ thesis, which will stored in the libraries of Victoria University of Wellington, University Sains Malaysia and may be available in other university libraries in Malaysia. It is also intended that the research will be published in academic journals and presented at national and international conferences. If you wish, a summary of the findings will be made available to you once the thesis has been completed.

\section{If you would like to have further information or you have concern about the research you can contact:}

\section{Researcher}

Che Rabiaah Mohamed

Graduate School of Nursing, Midwifery \& Health,

Victoria University of Wellington

PO Box 600

Wellington 6140, NEW ZEALAND

OR

445, KM 3, Jalan Pengkalan Chepa, 15400

Kota Bharu, Kelantan, MALAYSIA.

Tel 006097481023 (Malaysia)

006443862188 (Wellington).

\section{Supervisor}

Dr. Katherine Nelson, Senior Lecturer, Graduate School of Nursing, Midwifery \& Health,

Victoria University of Wellington, PO Box 600,

Wellington, 6140, NEW ZEALAND

Tel 006444636138

e-mail: kathy.nelson@vuw.ac.nz 


\title{
A study of the Educational Needs of Home-Based Stroke Patients and Family Caregivers in Malaysia
}

\author{
Researcher name: Che Rabiaah Mohamed
}

\section{Consent Form for Focus Group for Health Professionals}

I have read the information sheet and have had the details of the study explained to me. My questions have been answered to my satisfaction, and I understand that I may ask further questions at any time.

I understand that this study has been approved by the Victoria University of Wellington Human Ethics Committee and written approval has been obtained from Hospital University Sains Malaysia.

I agree to participate in this study and for the audio-taping of the focus group under the conditions set out in the Information Sheet.

I would like a copy of the research finding.

Yes No

Signature:

Date:

..

Contact details for research findings: 
Appendix 11:- Interview guide for focus group and individual interview for Phase 2 of study

\section{Introduction:}

\section{Interview Guide for Focus Group for Health Professionals}

Thank people for attending

Revisit the purpose of the focus group

Reiterate the focus group ground rules covered in interview schedule

An overview of the findings from Phase 1 related to education resource development will then be presented. These findings will include those from the stroke patients, caregivers and health professionals' interviews.

\section{Questions:}

1. Based on the findings that I had shared, establish what the health professionals consider are the priorities for developing educational resources for stroke patients and their caregivers?

2. Why are these important - that is how would addressing these give a positive impact on stroke patients and the caregivers? What impact would addressing these have on staff?

3. Would now like to discuss/develop possible implementation plans for each of these priorities:

- How can we implement these - such as what form should the resource take?

- Who should be involved and who should take the lead?

- How could participants and other parties such as community members or other organisations involved or contribute in the proposed implementations?

- What funding is required and where will this be obtained

- What timeframe should we aim for?

- How will we evaluate

- Where to from here

Thank people for participation 


\section{References}

Adams, H. P. (1998). Treating ischemic stroke as an emergency. Arch Neurol, 55(4), 457-461.

Addington-Hall, J. (1995). Symptom control, communication with health professionals, and hospital care of stroke patients in the last year of life as reported by surviving family, friends, and officials. Stroke, 26(12), 2242-2248.

Alaszewski, A., Alaszewski, H., Potter, J., \& Penhale, B. (2007). Working after a stroke: Survivors' experiences and perceptions of barriers to and facilitators of the return to paid employment. Disability and Rehabilitation, 29(24), 1858-1869.

Ali, A. (2001). Antenatal care in Malaysia: Maintaining traditional practice alongside western medicine. Unpublished $\mathrm{PhD}$ thesis, University of Otago, Wellington, New Zealand.

Anderson, C., Rubenach, S., Mhurchu, C. N., Clark, M., Spencer, C., \& Winsor, A. (2000). Hospital or home for stroke rehabilitation? Results of a randomized controlled trial. I: Health outcomes at 6 months. Stroke, 31(5), 1024-1031.

Anderson, C. S., Linto, J., \& Stewart-Wynne, E. G. (1995). A population-based assessment of the impact and burden of caregiving for long-term stroke survivors. Stroke, 26(5), 843-849.

Anderson, S., \& Marlett, N. J. (2004). Communication in stroke: The overlooked rehabilitation tool. Age and Ageing, 33(5), 440-443.

Appleton, J. V. (1995). Analysing qualitative interview data: Addressing issues of validity and reliability. Journal of Advanced Nursing, 22, 993-997.

Ariff, K. M., \& Beng, K. S. (2006). Cultural health belief in a rural family practice: A Malaysian perspectives. Australian Journal of Rural Health, 14(1), 2-8.

Ariff, K. M., \& Lieng, T. C. (2002). Rural health care in Malaysia. Australian Journal of Rural Health, 10(2), 99-103.

Asbury, J. E. (1995). Overview of focus group research. Qualitative Health Research, $5(4), 414-420$.

Astrom, M., Adoffson, R., \& Asplund, K. (1993). Major depression in stroke patients: A 3-year longitudinal study. Stroke, 24(7), 976-982.

Attride-Stirling, J. (2001). Thematic networks: An analytic tool for qualitative research. Qualitative Research, 1(3), 385-405.

Auslander, G. K., \& Gold, N. (1999). Disability terminology in the media: A comparison of newspaper report in Canada and Israel. Social Science \& Medicine, 48(10), 1395-1405.

Australia National Stroke Foundation (2005). Clinical guidelines for stroke rehabilitation and recovery. National health and medical research council. Retrieved 28 October 2009. from http://www.nhmrc.gov.au/-filesnhmrc/file/publications/synopses/cp105.pdf.

Bakas, T., Austin, J. K., Jessup, S. L., Williams, L. S., \& Oberst, M. T. (2004). Time and difficulty of tasks provided by family caregivers of stroke survivors. Journal of Neuroscience Nursing, 36 (2), 95-106.

Bakas, T., Austin, J. K., Okonkwo, K. F., Lewis, R. R., \& Chadwick, L. (2002). Needs, concerns, strategies, and advice of stroke caregivers the first 6 months after discharge. Journal of Neuroscience Nursing, 34(5), 242-251. 
Bendz, M. (2003). The first year of rehabilitation after a stroke-from two perspectives. Scandinavian Journal of Caring Science, 17(3), 215-222.

Bennet, B. (1996). How nurses in stroke rehabilitation unit attempt to meet the psychological needs of patients who become depressed following a stroke. Journal of Advanced Nursing, 23(2), 314-321.

Berg, B. L. (2007). Qualitative research method for the social sciences (6th ed.). New York: Pearson Education, Inc.

Bhogal, S. K., Teasel, R. W., Foley, N. C., \& Speechley, M. R. (2003a). Rehabilitation of aphasia. Top Stroke Rehabil, 10(2), 66-76.

Bhogal, S. K., Teasel, R. W., Foley, N. C., \& Speechley, M. R. (2003b). Community reintegration after stroke. Top Stroke Rehabil, 10(2), 107-129.

Birks, M. J., Chapman, Y., \& Francis, K. (2007). Breaching the wall: Interviewing people from other cultures. Journal of Transcultural Nursing 18(2), 150-156.

Bluvol, A., \& Gilboe, M. F. (2004). Hope, health work and quality of life in families of stroke survivors. Journal of Advanced Nursing, 48(4), 322-332.

Bodenheimer, T., Wagner, E. H., \& Grumbach, K. (2002). Improving primary care for patients with chronic illness: The chronic care model, part 2. Journal of American Medical Association, 28(15), 1909-1914.

Bonita, R. (2004). The global stroke initiative. The Lancet, 3(7), 391-393.

Boughton, M., \& Halliday, L. (2009). Home alone: Patient and carer uncertainty surrounding discharge with continuing clinical care needs. Contemporary Nurse, 33(1), 30-40.

Bowling, A. (1997). Research method in health: Investigating health and health services. Buckingham: Open University Press.

Brashler, R. (2006). Ethics, family caregivers and stroke. Topics in Stroke Rehabilitation, 13(14), 11-17.

Braun, V., \& Clarke, V. (2006). Using thematic analysis in psychology. Qualitative Research in Psychology, 3, 77-101.

Brereton, L. (1997). Preparation for family care-giving: Stroke as a paradigm case. Journal of Clinical Nursing, 6(6), 435-424.

Brereton, L., \& Nolan, M. (2000). 'You do know, he's had a stroke don't you? preparation for family care-giving: The neglected dimension. Journal of Clinical Nursing, 9(4), 498-506.

Brereton, L., \& Nolan, M. (2002). 'Seeking': A key activity for new family carers of stroke survivors. Journal of Clinical Nursing, 11(1), 22-31.

Broderick, J. P. (2004). William M. Feinberg lecture: Stroke therapy in the year 2025. Burden, breakthrough, and barriers in progress. 35, 205-211.

Bryman, A., \& Burgess, R. G. (1994). Analyzing Qualitative Data. London: Routledge.

Bugge, C., Alexander, H., \& Hagen, S. (1999). Stroke patients' informal caregivers. Patient, care and service factors that affect caregivers strain. Stroke, 30(8), 15171523.

Burnard, P. (2005). Interviewing. Nurse Researcher, 13(1), 4-6.

Burns, N., \& Grove, S. K. (2005). The practice of nursing research. conduct, critique, \& utilization (5th ed.). Philadelphia: W.B Saunders Company.

Burns, N., \& Grove, S. K. (2007). Understanding nursing research: Building an evidence-based practice (4th ed.). Missouri: Saunders Elsevier. 
Burton, C. (2000). Living with stroke: A phenomenological study. Journal of Advanced Nursing, 32(2), 301-309.

Butcher, J., \& Frankish, H. (2006). Stroke: A call for papers. The Lancet, 368, 976.

Cadilhac, D. A., Ibrahim, J., Pearce, D. C., Ogden, K. J., McNeill, J., Davis, S. M., et al. (2004). Multicenter comparison of processes of care between stroke units and conventional care wards in Australia. Stroke, 35(5), 1035-1040.

Cameron, J. I., \& Gignac, M. A. M. (2008). "Timing it right": A conceptual framework for addressing the support needs of family caregivers to stroke survivors from the hospital to the home. Patient education and Counseling, 70(3), 305-314.

Chemerinski, E. (2001). Improved recovery in activities of daily living associated with remission of post-stroke depression. Stroke, 32(1), 113-117.

Choi-Kwon, S., \& Kim, J. S. (2002). Poststroke emotional incontinence and decrease sexual activity. Cerebrovascular Disease, 13(1), 31-37.

Choi-Kwon, S., Lee, S. K., Park, H. A., Kwon, S. U., Ahn, J. S., \& Kim, J. S. (2005). What stroke patients want to know and what medical professionals think they should know about stroke: Korean perspectives. Patient Education and Counseling, 56(1), 85-92.

Clarke, P. (2002). Well-being after stroke in Canadian seniors: Findings from the Canadian study of health and aging. Stroke, 33(4), 1016-1021.

Cloutier-Fisher, D. S. (2005). Different Strokes. Need for help among stroke-affected persons in British Columbia. Canadian Journal of Public Health, 96(3), 221-225.

Coleman, M. T., \& Newton, K. S. (2005). Supporting self-management in patients with chronic illness. American Family Physician, 72(8), 1503-1510.

Conroy, B. E., Zorowitz, R. D., Milani, F., Bartlett, C., Tyner, K., Stein, J., et al. (2009). Challenges in community rehabilitation Stroke recovery and rehabilitation (pp. 747-765). New York: Demos Medical Publishing.

Coolican, H. (2004). Research method \& statistics in psychology (4th ed.). London: Hodder \& Stoughton.

Coombs, U. E. (2007). Spousal caregiving for stroke survivors. Journal of Neuroscience Nursing, 39(2), 112-119.

Corr, S., \& Wilmer, S. (2003). Returning to work after a stroke: An important but neglected area. The British Journal of Occupational Therapy, 66(5), 186-192.

Coulter, A., \& Ellins, J. (2007). Effectiveness of strategies for informing, educating and involving patients. British Medical Journal, 335(7609), 24-27.

Curtis, E., \& Redmond, R. (2007). Focus groups in nursing research. Nurse Researcher, 14(2), 25-37.

Daniel, K., Wolfe, C. D. A. Busch, M. A., \& McKevitt, C. (2009). What are the social consequences of stroke for working-aged adults? A systematic review. Stroke, 40(6), e431-440.

Dearnley, C. (2005). A reflexion on the use of semi-structured interviews. Nurse Researcher, 13(1), 19-28.

Denby, F., \& Harvey, R. L. (2003). An educational intervention for stroke rehabilitation patients and their families: Healthy living after stroke. Topic in Stroke Rehabilitation, 9(4), 34-45.

Dobkin, B. H. (2005). Rehabilitation after stroke. The New England Journal of Medicine, 352(16), 1677-1785. 
Dorsey, M. K., \& Vaca, K. J. (1998). The stroke patient and assessment of caregiver needs. Journal of Vascular Nursing, 16(3), 62-67.

Downswell, G. (2000). Investigating recovery from stroke. A qualitative study. Journal of Clinical Nursing, 9(4), 507-515.

Eames, S., Hoffman, T., McKenna, K., \& Worral, L. (2008). Community-based stroke information for clients with stroke and their carers: Is there congruency between actual and recommended practice? Topic in Stroke Rehabilitation, 15(4), 295-306.

Eames, S., McKenna, K., Worral, L., \& Reed, S. (2003 ). The suitability of written education materials for stroke survivors and their carers. Topics in Stroke Rehabilitation, 10(3), 70-83.

Eaves, Y. D. (2000). "What happened to me": Rural African American elders' experiences of stroke. Journal of Neuroscience Nursing, 32(1), 37-48.

Edwards, L. (2009). Stroke education sessions in a rehabilitation unit. The official journal of the Australian Rehabilitation Nurses' Association, 12(3), 4-6.

Ellis-Hill, C., Robison, J., Wiles, R., McPherson, K., Hyndman, D., Ashburn, A., et al. (2009). Going home to get on with life: Patients and carers experiences of being discharged from hospital following a stroke. Disability and Rehabilitation, 31(2), 61-72.

Endacott, R. (2005). Clinical research 4: Qualitative data collection. Intensive and Critical Care Nursing, 21(2), 123-127.

Epstein-Lubow, G. P., Beevers, C. G., Bishop, D, S., Miller, I. W. (2009). Family functioning is associated with depressive symptoms in caregivers of acute stroke survivors. Archives of Physical Medicine and Rehabilitation, 90(6), 947-955.

Errikson, M., Asplund, K., Glader, E. L., Norrving, B., Stegmayr, B., Terent, A., et al. (2004). Self-reported depression and use of antidepressant after stroke: A national survey. Stroke, 35(4), 936-941.

Exall, K. (1999). Caring for carers coping with stroke. Nursing Times, 95(11), 50-51.

Faircloth, C. A., Boylstein, C., Rittman, M., \& Gubrium, J. F. (2005). Constructing the stroke: Sudden-onset narratives of stroke survivors. Qualitative Health Research, 15(7), 928-941.

Farzan, D. T. (1991). Reintegration for stroke survivors. Home and community reintegration. Nursing Clinics of North America, 26(4), 1037-1048.

Fern, E. F. (2001). Advanced focus group research. Thousand Oaks: Sage Publications.

Forsberg-Warleby, G., Moller, A., \& Blomstrand, C. (2002). Spouses of first-ever stroke patients, their view of the future during the first phase after stroke. Clinical Rehabilitation, 16(5), 506-514.

Forster, A., \& Young, J. (1996). Specialist nurse supports for patients with stroke in the community: A randomized controlled trial. British Medical Journal, 312(7047), 1642-1646.

Freeman, T. (2006). 'Best practice' in focus group research: Making sense of different views. Journal of Advanced Nursing, 56(5), 491-497.

Fuh, J. L. (1997). Poststroke depression among the Chinese elderly in a rural community. Stroke, 28, 1126-1129.

Garret, D., \& Cowdell, F. (2005). Information needs of patients and carers following stroke. Nursing Older People, 17(6), 14-16. 
Gibbon, B. (1994). Stroke nursing care and management in the community: A survey of district nurses' perceived contribution in one health district in England. Journal of Advanced Nursing, 20(3), 469-476.

Glamcevski, M. T., \& Tan, C. T. (2000). Prevalence of post-stroke depression: A Malaysian study. Neurol J Southeast Asia, 5, 51-53.

Glamcevski, T. M. (2002). Factors associated with post-stroke depression: A Malaysian study. Neurol J Southeast Asia, 7, 9-12.

Glasgow, R. E., Orleans, C. T., Wagner, E. H., Curry, S. J., \& Solberg, L. I. (2001). Does the Chronic Care Model serve also as a template for improving prevention? The Milbank Quarterly, 79(4), 579-612.

Goulding, R., Thompson, D., \& Beech, C. (2004). Caring for patients with hemiplegia in an arm following a stroke. British Journal of Nursing, 13(9), 534-539.

Grant, J. S., \& Davis, L. L. (1997). Living with loss: The stroke family caregiver. Journal of Family Nursing, 3(1), 36-56.

Grbich, C. (1999). Qualitative research in health: An introduction. New South Wales: Allen \& Unwin Pty Ltd.

Green, T. L., \& King, K. M. (2007). The trajectory of minor stroke recovery for men and their female spousal caregivers: Literature review. Journal of Advanced Nursing, 58(6), 517-531.

Green, T. L., \& King, K. M. (2009). Experiences of male patients and wife-caregivers in the first year post-discharge following minor stroke: A descriptive qualitative study. International Journal of Nursing Studies, 46(9), 1194-1200.

Gubrium, J. F., \& Holstein, J. A. (2002). Handbook of Interviewing. California: SAGE.

Gupta, A., \& Thomas, P. (2002). Knowledge of stroke symptoms and risk factors among at-risk elderly patients in the UK. Int J Clin Prac, 56(9), 634-637.

Hafteinsdottir, T. B., \& Grypdonck, M. (1997). Being a stroke patient: A review of the literature. Journal of Advanced Nursing, 26(3), 580-588.

Hamidon, B. B., \& Raymond, A. A. (2003). The impact of diabetes mellitus on inhospital stroke mortality. Journal of Postgraduate Medicine, 49(4), 307-310.

Han, B., \& Haley, W. E. (1999). Family caregiving for patients with stroke: Review and analysis. Stroke, 30, 1478-1485.

Hanafiah, M. (1996). Public health care provisions: Access and equity. Social Science Medicine, 43(5), 759-768.

Hanger, H. C., \& Mulley, G. P. (1993). Questions people ask about stroke. Stroke, 24(4), 536-538.

Hanger, H. C., Walker, G., \& Paterson, L. A. (1998). What do patients and their carers want to know about stroke? A two-year follow-up study. Clinical Rehabilitation, 12(1), 45-52.

Hanger, H. C., \& Wilkinson, T. J. (2001). Stroke education: Can we rise to the challenge. Age and ageing, 30(2), 113-114.

Hare, H., Rogers, H., Lester, H., McManus, R. J., \& Mant, J. (2006). What do stroke patients and their carers want from community services? Family Practice, 23(1), 131-136.

Hayn, M. A., \& Fisher, T. R. (1997). Stroke rehabilitation: Salvaging ability after the storm. Nursing, 27(3), 63-70. 
Heeley, E., Anderson, C. S., Huang, Y., Jan, S., Li, Y., Liu, M et al. (2009). Role of health insurance in averting economic hardship in families after acute stroke in China. Stroke, 40(6), 2149-2156.

Henderson, A., Korner-Bitensky, N., \& Levin, M. (2007). Virtual reality in stroke rehabilitation: A systematic review of its effectiveness for upper limb motor recovery. Top Stroke Rehabil, 14(2), 52-61.

Hickey, J. V. (2001). The clinical practice of neurological and neurosurgical nursing. New York: JB Lippincott Company.

Hickey, J. V. (2009). The clinical practice of neurological and neurosurgical nursing (6th ed.). New York: Lippincott Williams and Wilkins.

Hinojosa, M. S., \& Rittman, M. (2009). Association between health education needs and stroke caregiver injury. Journal of Aging and Health, 21(7), 1040-1058.

Hoffmann, T., \& Cochrane, T. (2009). What education do stroke patients receive in Australian hospital? Patient Education and Counseling, 77(2), 187-191.

Hoffmann, T., \& McKenna, K. (2006). Analysis of stroke patients' and carers' reading ability and the content and design of written materials: Recommendations for improving written stroke information. Patient Education and Counseling, 60(3), 286-293.

Hoffmann, T., McKenna, K., Herd, C., \& Wearing, S. (2007). Written education materials for stroke patients and their carers: Perspectives and practices of health professionals. Top Stroke Rehabil, 14, 88-97.

Hopmann, W. M., \& Verner, J. (2003). Quality of life during and after inpatient stroke rehabilitation. Stroke, 34(3), 801-805.

Holloway, I., \& Wheeler, S. (1996). Qualitative research for nurses. London: Blackwell Science Ltd.

Hroscikoski, M. C., Solberg, L.I., Sperl-Hillen, J. M., Harper, P. G., McGrail, M.P., \& Crabtree, B.F. (2006). Challenges of change: A qualitative study of Chronic Care Model implementation. Annals of Family Medicine 4:317-326.

Hung, D. Y., Rundall, T. G., Tallia, A. F., Cohen, D. J., \& Crabtree, B. F. (2007). Rethinking prevention in primary care: Applying the Chronic Care Model to address health risk behaviours. The Milbank Quarterly, 85(1), 69-91.

Hutchinson, S., \& Wilson, H. S. (1992). Validity threats in scheduled semistructured research interviews. Nursing Research, 41(2), 117-119.

Ismail, Z., Mohamed, R., Mohd, H. M. H., \& Wan, S. K. (2005). Usage of traditional medicine among elderly and the prevalence of prednisolone contamination. Malaysian Journal of Medical Science, 12(2), 50-55.

Jaya, F., Win, M. N., Abdullah, M. R., Abdullah, M. R., \& Abdullah, J. M. (2002). Stroke patterns in Northeast Malaysia: A hospital-based prospective study. Neuroepidemiology, 21(1), 28-35.

Jester, R., \& Hicks, C. (2003). Using cost-effectiveness analysis to compare hospital at home and in-patient interventions. Part 1. Journal of Clinical Nursing, 12, 13-19.

Johnson, A., \& Sandford, J. (2005). Written and verbal information versus verbal information only for patients being discharged from acute hospital settings to home: A systematic review. Health Education Research, 20(4), 423-429.

Johnson, P. D. (1998). Rural stroke caregivers: A qualitative study of the positive and negative response to the caregiver's role. Topics in Stroke Rehabilitation, 5, 51-68. 
Jonsson, A. C., Lindgren, I., Hallstrom, B., Norrving, B., \& Lingren, A. (2005).

Determinants of QOL in stroke survivors and their informal caregivers. Stroke, 36, 803-808.

Jorgensen, H. S., Nakayama, H., Reith, J., Raaschou, H. O., \& Olsen, T. S. (1996). Factors delaying hospital admission in acute stroke: The Copenhagen Stroke Study. Neurology, 47, 383-387.

Jullamate, P., Azeredo, Z. D., Paul, C., \& Subgranon, R. (2006). Thai stroke patient caregivers: Who they are and what they need. Cerebrovascular Disease, 21(1-2), 128-133.

Jullamate, P., de Azeredo, Z., Paul, C., \& Subgranon, R. (2006). Informal stroke rehabilitation: What do Thai caregivers perform. International Journal of Rehabilitation Research, 29(4), 309-314.

Kalra, L., Evans, A., Perez, I., Melbourn, A., Patel, A., Knapp, M., et al. (2004). Training carers of stroke patients: Randomized controlled trial. British Medical Journal, 328(7448), 1099-1101.

Kim, J. S., \& Yoon, S. S. (1997). Perspectives of stroke in persons living in Seoul, South Korea. A survey of 1000 subjects. Stroke, 28(6), 1165-1169.

Kimura, M., Murata, Y., Shimoda, K., \& Robinson, R. G. (2001). Sexual dysfunction following stroke. Comprehensive Psychiatry, 42(3), 217-222.

Kitzinger, J. (1994). The methodology of focus group: The importance of interaction between research participants. Sociology of Health and Illness, 16(1), 103-121.

Kitzinger, J. (1995). Introducing focus group. British Medical Journal, 311(7000), 299302.

Kitzinger, J., \& Barbour, R. S. (2001). Introduction: The challenge and promise of focus groups In R. S. Barbour \& J. Kitzinger (Eds.), Developing focus group research: Politics, theory and practice (2nd ed., pp. 1-20). London: SAGE Publications Ltd.

Knight, K., Worral, L., \& Rose, T. (2006). The provision of health information to stroke patients within an acute hospital setting: What actually happens and how do patient feel about it? Topics in stroke Rehabilitation, 13(1), 78-97.

Koch, L., Egbert, N., Coeling, H., \& Ayers, D. (2005). Returning to work after the onset of illness: Experience of right hemisphere stroke survivors. Rehabilitation Counseling Bulletin, 48(4), 209-218.

Korff, M. V., Gruman, J., Schaefer, J., Curry, S. J., \& Wagner, E. H. (1997). Collaborative management of chronic illness. Annals of Internal Medicine, 10971102.

Korpelainen, J. T., Kauhanen, M. L., Kemola, H., Malinen, U., \& Myllyla, V. V. (1998). Sexual dysfunction in stroke patients. Acta Neurol Scand, 98(6), 400-405.

Korpelainen, J. T., Niemen, P., \& Myllyla, V. V. (1999). Sexual functioning among stroke patients and their spouses. Stroke, 30(4), 715-719.

Kothari, R., Sauerbeck, L., Jauch, E., Broderick, J., Brott, T., Khoury, J., et al. (1997). Patients' awareness of stroke signs, symptoms, and risk factors. Stroke, 28(10), 1871-1875.

Krishnaswamy, S., Subramaniam, K., Low, W. Y., Aziz, J. A., Indran, T., Ramachandran, P., et al. (2009). Factors contributing to utilization of health care services in Malaysia: A population based study. Asia-Pacific J Public Health, 21(4), 442450. 
Krueger, R. A. (1994). A practical guide for applied research (2nd ed.). California: Sage Publications.

Krueger, R. A. (1995). The future of focus group. Qualitative health research, 5(4), 524530.

Kvale, S. (1996). Interviews. An introduction to qualitative research interviewing. London: Sage Publications.

Lam, T. P., Irwin, M., \& Chow, L. W. C. (2001). The use of focus group interviews in Asian medical education evaluative research. Medical Education, 35(5), 510-513.

Langhorne, P., \& Legg, L. (2003). Evidence behind stroke rehabilitation. J Neurol Neurosurg Psychiatry, 74, 18-21.

Langhorne, P., \& Rudd, A. G. (2009). Stroke services: A global perspectives. In J. Stein, R. L. Harvey \& R. F. Macko (Eds.), Stroke recovery and rehabilitation (pp. 597606). New York: Demos Medical Publishing.

Lapadat, J. C., \& Lindsay, A. C. (1999). Transcription in research and practice: From standardization of technique to interpretive positionings. Qualitative Inquiry, 5(1), 64-86.

Lateef, F., \& Anantharaman, V. (2005). Brain attack: The multifaceted potential for action Singapore Medical Journal, 39(7). Retrieved from http://www.sma.org.sg/smj/3907/articles/3907a5.htm

Lees, K. R., Bath, P. M. W., \& Naylor, A. R. (2000). 'ABC of arterial and venous disease: Secondary prevention of transient ischaemic attack and stroke'. British Medical Journal, 320, 991-994.

Leng, C. H., \& Barraclough, S. (2007). Health care in Malaysia: The dynamic of provision, financing and access. London: Routledge.

Liechty, J. A., \& Braun, M. E. (2006). Loss and hope: Strategies for coping with aphasia. Top Stroke Rehabil, 13(3), 84-86.

Liechty, J. A., \& Garber, D. W. (2004). Dealing with aphasia: Three simple rules. Rehabilitation Nursing, 29(1), 3-4.

Lincoln, N. B., Parry, R. H., \& Vass, C. D. (1999). Randomized controlled trial to evaluate increased intensity of physiotherapy treatment of arm function after stroke. Stroke, 30(3), 573-579.

Lincoln, N. B., Nicholl, C. R., Flannaghan, T., Leonard, M., \& Van der Gucht, E. (2003). The validity of questionnaire measures for assessing depression after stroke. Clinical Rehabilitation, 17(8), 840-846.

Litosseliti, L. (2005). Using focus groups in research. London: Continuum.

Lott, C., Hennes, J. H., \& Dick, W. (1999). Stroke - a medical emergency. Journal of Accident \& Emergency Medical, 16, 2-12.

Lutz, B. J., Chumbler, N. R., \& Roland, K. (2007). Care coordination/home-telehealth for veterans with stroke and their caregivers: Addressing an unmet need. Topics in Stroke Rehabilitation, 14(2), 32-43.

Mackenzie, A. E., Holroyd, E. E., \& Lui, H. L. (1998). Community nurse' assessment of the needs of Hong Kong family carers who are looking after patients. International Journal of Nursing Studies, 35(3), 132-140.

MacLean, L. M., Meyer, M., \& Estable, A. (2004). Improving accuracy of transcripts in qualitative research. Qualitative Health Research, 14(1), 113-123. 
Man, K., Kareem, A. M. M., Ahmad Alias, N. A., Tharakan, J., Abdullah, J. M., Prasad, A., et al. (2006). Computed tomography perfusion of ischaemic stroke patients in rural Malaysian tertiary referral centre. Singapore Medical Journal, 47(3), 194197.

Mant, J., Carter, J., Wade, D. T., \& Winner, S. (2000). Family support for stroke: A randomised controlled trial. The Lancet, 356(9232), 801-813.

Martin, L. G. (1989). Living arrangements of the elderly in Fiji, Korea, Malaysia and the Philippines. Demography, 26(4), 627-644.

Mayo, N. E., Dauphinee, S. W., Cote, R., Gayton, D., Carlton, J., Buttery, J., et al. (2000). There's no place like home. An evaluation of early supported discharge for stroke. Stroke, 31(5), 1016-1023.

Mays, N., \& Pope, C. (1995). Rigour and qualitative research. British Medical Journal, 311(6997), 109-112.

Mays, N., \& Pope, C. (2000). Assessing quality in qualitative research. British Medical Journal, 320(7226), 50-52.

McBride, K. L., White, C. L., Sourial, R., \& Mayo, N. (2004). Postdischarge nursing interventions for stroke survivors and their families. Journal of Advanced Nursing, 47(2), 192-200.

McCann, L., Groot, P., Charnley, C., \& Gardner, A. (2009). Excellence in regional stroke care: An evaluation of the implementation of a stroke care unit in regional Australia. Australian Journal of Rural Health, 17(5), 273-278.

Mcevoy, P., \& Barnes, P. (2007). Using the chronic care model to tackle depression among the older adults who have the long-term physical conditions. Journal of Psychiatric and Mental Health Nursing, 14, 233-238.

Mclean, J., Roper-Hall, A., Mayer, P., \& Main, A. (1991). Service needs of stroke survivors and their informal carers: A pilot study. Journal of Advanced Nursing, 16(5), 559-564.

Merican, H. I., \& Yon, R. (2002). Health care reform and changes: The Malaysian experience. Asia-Pacific J Public Health, 14(1), 17-22.

Miles, M. B., \& Huberman, M. A. (1994). An expanded sourcebook: Qualitative data analysis (2nd ed.). London: Sage Publication.

Minichiello, V. (1991). In-depth interviewing. Researching people. Melbourne: Longman Cheshire.

Minichiello, V., Aroni, R., \& Hays, T. (2008). In-depth Interviewing. Principles, techniques, analysis (3rd ed.). Sydney: Pearson Education Australia.

Ministry of Health (2006). Clinical practice guidelines: Management of stroke. Retrieved 10 August, 2007. from http://www.malaysiaheart.org.

Ministry of Health Malaysia (2005). Annual Report. Kuala Lumpur: Author.

Ministry of Health Malaysia (2006). Annual report. Kuala Lumpur: Author.

Ministry of Health Malaysia (2008). Health Facts 2008. Retrieved 24 September, 2009. from http://www.moh.gov.my/opencms/export/sites/default/moh.

Mitchell, E., \& Moore, K. (2004). Stroke: Holistic care and management. Nursing Standard, 18(33), 43-52.

Mitchell, P. H., Teri, L., Veith, R., Buzaitis, A., Tirschwell, D., Becker, K et al., (2008). Living well with stroke: Design and methods for randomized controlled trial of a 
psychosocial behavioural intervention for postroke depression. Journal of Stroke and Cerebrovascular Disease, 17(3), 109-115.

Mohamed, C. R. (1998). Komunikasi pesakit neuro di hospital (Communication among neuro patients at a hospital). Unpublished Masters thesis, University of Malaya, Kuala Lumpur, Malaysia.

Morgan, D. L. (1993). Qualitative content analysis. Qualitative Health Research, 3(1), 112-121.

Morgan, D. L. (1995). Why things (sometimes) go wrong in focus groups. Qualitative Health Research, 5(4), 516-523.

Morse, J. M., \& Field, P. A. (1996). Nursing Research. The application of qualitative approaches. London: Chapman \& Hall.

Myers, D. G. (2002). Social Psychology (7th ed.). Michigan: Mc Graw Hill.

National Institute of Health (2006). Health Research Priorities. 9th Malaysia Plan 20062010. Kuala Lumpur: Author.

National Stroke Association of Malaysia (2007). Stroke in Malaysia. Retrieved 9 February, 2007, from www.nasam.org/prevention-what_is_a_stroke.php

Nik Rosnah, W. A. (2005). Regulating the private health sector in Malaysia. Kuala Lumpur: University of Malaya Press.

Nyamathi, A., \& Shuler, P. (1990). Focus group interview: A research technique for informed nursing practice. Journal of Advanced Nursing, 15(11), 1281-1288.

O'Connell, B., Baker, L., \& Prosser, A. (2003). The educational needs of caregivers of stroke survivors in acute and community settings. Journal of Neuroscience Nursing, 35(1), 21-30.

O'Connell, B., Penney, W., \& Owen, M. (2001). Recovery after stroke: A qualitative perspective. J. Qual. Clin. Practice, 21, 120-125.

Oloffson, A., Anderson, S. O., \& Carlberg, B. (2005). 'If only I manage to get home I'll get better' - Interviews with stroke patients after emergency stay in hospital on their experiences and needs. Clinical Rehabilitation, 19(4), 433-440.

O'Mahoney, P. G., Rodgers, H., Thomson, R. G., Dobson, R., \& James, O. F. (1997). Satisfaction with information and advice received by stroke patients. Clinical Rehabilitation, 11(1), 68-72.

Ong, T. Z., \& Raymond, A. A. (2002). Risk factors for stroke and predictors of onemonth mortality. Singapore Medical Journal, 43(10), 517-521.

Pancioli, A., Broderick, J., Kothari, R., Brott, T., Tuchfarber, A., Miller, R., et al. (1997). Public perception of stroke warning signs and potential risk factors. JAMA, 279, 1288-1292.

Penington, G. R., \& Burry, H. C. (1990). Introduction to medical rehabilitation. Melbourne: Melbourne University Press.

Perry, L. (2001). Screening swallowing function of patients with acute stroke. Part one: Identification, implementation and initial evaluation of a screening tool for use by nurses. Journal of Clinical Nursing, 10, 463-473.

Perry, L., \& McLaren, S. (2003). Eating difficulties after stroke. Journal of Advanced Nursing, 43(4), 360-369.

Pierce, L. L., Finn, M. G., \& Steiner, V. (2004). Families dealing with desire information about self-care needs. Rehabilitation Nursing, 29(1), 14-17. 
Pierce, L. L., Steiner, V., Govoni, A. L., Hicks, B., Cervantez Thompson, T. L., \& Friedemann, M. L. (2004). Caregivers dealing with stroke pull together and feel connected. Journal of Neuroscience Nursing, 36(1), 32-39.

Pierce, L. L., Steiner, V., Hicks, B., \& Holzaedfel, A. L. (2006). Problems of new caregivers of persons with stroke. Rehabilitation Nursing, 31(4), 166-172.

Poi, P. J.-H., Forsyth, D. R., \& Chan, D. K. Y. (2004). Services for older people in Malaysia: issues and challenges. Age and Ageing, 33(5), 444-446.

Polit, D. F., \& Hungler, B. P. (1993). Essentials of nursing research. Methods, appraisal, and utilization (3rd ed.). Philadelphia: J. B Lippincott Company.

Pollack, M. R. P., \& Disler, P. B. (2002). Rehabilitations of patient after stroke. Medical Journal of Australia, 177(8), 444-448.

Pope, C., Ziebland, S., \& Mays, N. (2000). Qualitative research in healthcare: Analysing qualitative data. British Medical Journal, 320(7227), 114-116.

Pound, P. (1999). Does patient satisfaction reflect differences in care received after stroke? Stroke, 30(1), 49-55.

Pound, P., Gompertz, P., \& Ebrahim, S. (1999). Social and practical strategies described by people living at home with stroke. Health and Social Care in the Community, $7(2), 120-128$.

Rahman, A. A., Sulaiman, S. A., Ahmad, Z., Daud, W. N. W., \& Hamid, A. M. (2008). Prevalence and pattern of use of herbal medicines during pregnancy in Tumpat district, Kelantan. Malaysian Journal of Medical Science, 15(3), 40-48.

Ramli, A. S., \& Taher, S. W. (2008). Managing chronic diseases in the Malaysian primary health care - A need for Change. Malaysian Family Physician, 3(1), 7-13.

Rausch, M., \& Turkoski, B. (1999). Developing realistic treatment standards in today's economic climate: Stroke survivor education. Journal of Advanced Nursing, 30(2), 329-334.

Razali, S. M. (2009). Integrating Malay traditional healers into Primary Health Care services in Malaysia: Is it possible? International Medical Journal, 16(1), 13-17.

Reza, M. F., Urakami, Y., \& Mano, Y. (2002). Medical benefits of salat. Annals of Saudi Medicine, 22, 3-4.

Robinson, C. A., \& Wright, L. M. (1995). Family nursing intervention: What families say makes a difference. Journal of Family Nursing, 1(3), 327-345.

Rodgers, H., Atkinson, C., Bond, S., Suddes, M., Dobson, R., \& Curless, R. (1999). Randomized controlled trial of a comprehensive stroke education program for patients and caregivers. Stroke, 30(12), 2585-2591.

Rombough, R. E., Howse, E. L., Bagg, S. D., \& Bartfay, W. J. (2007). A comparison of studies on the quality of life of primary caregivers of stroke survivors: A systematic review of the literature. Top Stroke Rehabil, 14(3), 69-79.

Roomruangwong, C., \& Thavichachart, N. (2005). Prevalence of anxiety after stroke in physical rehabilitation patients in King Chulalongkorn Memorial Hospital. Chula Med Journal, 49(4), 213-223.

Rose, T. A., Worral, L. E., McKenna, K. T., Hickson, L. M., \& Hoffmann, T. C. (2009). Do people with aphasia receive written stroke and aphasia information. Aphasiology, 23(3), 364-392.

Royal College of Physician (2004). National Clinical Guidelines for Stroke., from http://www.strokeupdate.co.uk/stroke_guidelines_2ed[1].pdf $\geq$ 
Ryan-Nicholls, K. D., \& Will, C. I. (2009). Rigour in qualitative research. Nurse Researcher, 16(3), 70-85.

Samsa, G. P., Cohen, S. J., \& Goldstein, S. B. (1997). Knowledge of risk among patients at increased risk of stroke. Stroke, 28(5), 916-921.

Sandelowski, M. (1986). The problem of rigor in qualitative research. Advances in Nursing Science, 8(3), 27-37.

Sandelowski, M. (1995a). Sample size in qualitative research. Research in Nursing \& Health, 18(2), 179-183.

Sandelowski, M. (1995b). Qualitative analysis: What it is and how to begin. Research in Nursing \& Health, 18(4), 371-375.

Sandelowski, M. (2000). Whatever happened to qualitative description? Research in Nursing \& Health, 23(4), 334-340.

Secrest, J. (2000). Transformation of the relationship: The experience of primary support persons of stroke survivors. Rehabilitation Nursing, 25(3), 93-98.

Silverman, D. (1997). Qualitative research: Theory, method and practice. London: Sage publications.

Sim, J. (1998). Collecting and analysing qualitative data: Issues raised by the focus group. Journal of Advanced Nursing, 28(2), 345-352.

Ski, C., \& O'Connel, B. (2007). Stroke: The increasing complexity of carer needs. Journal of Neuroscience Nursing, 39(3), 172-179.

Smith, D. S., Gignac, M. A. M., Richardson, D., \& Cameron, J. I. (2008). Differences in the experiences and support needs of family caregivers to stroke survivors: Does age matter? Top Stroke Rehabilitation, 15(6), 593-601.

Smith, L. N., Lawrence, M., Kerr, S. M., Langhorne, P., \& Lees, K. R. (2004). Informal carers' experience of caring for stroke survivors. Journal of Advanced Nursing, 46(3), 235-244.

Smith, M. W. (1995). Ethics in Focus Groups: A few concerns. Qualitative Health Research, 5(4), 478-486.

Snape, D., \& Burton, C. (2002). Stroke rehabilitation: An agenda for development. Nursing Standard, 17(3), 35-38.

Solberg, L. I., Crain, A. L., Sperl-Hillen, J. M., Hroscikoski, M. C., Engebretson, K. I., \& O'Connor, P. J. (2006). Care quality and implementation of the Chronic Care Model: A quantitative study. Annals of Family Medicine, 4(4), 310-316.

Somerfield, J., Barber, P. A., Anderson, N. E., Spriggs, D., Charleston, A., \& Bennet, P. (2006). Changing attitude to the management of ischaemic stroke between 1997 and 2004: A survey of New Zealand Physicians. Internal Medicine Journal, 36(5), 276-280.

Streubert, H. J., \& Carpenter, D. R. (1999). Qualitative research in nursing. Advancing humanistic imperative (2nd ed.). Philadelphia: Lippincott Williams \& Wilkins.

Sundin, K., Jansson, L., \& Norberg, A. (2000). Communicating with people with stroke and aphasia: Understanding through sensation without words. Journal of Clinical Nursing, 9(4), 481-488.

Talbot, R. L., Viscogliosi, C., Desrosiers, J., Vincent, C., Rousseau, J., \& Robichaud, L. (2004). Identification of rehabilitation needs after a stroke : An exploratory study. Health and Quality of Life Outcomes, 2(53). Retrieved from http://www.hqlo.com/content/2/1/53 
Tan, K. S., Wong, K. S., \& Venketsubramaniam, N. (2006). Setting priorities in Asian stroke research. Neurology Asia, 11, 5-11.

Tatemichi, T. K., Desmond, D. W., Stern, Y., Paik, M., Sano, M., \& Bagiella, E. (1994). Cognitive impairment after stroke: Frequency, patterns, and relationship to functional abilities. Journal of Neurology, Neurosurgery and Psychiatry, 57(2), 202-207.

The Star Online (2007, 18 July 2009). Kepala Batas Hospital first to integrate traditional and modern medicine. The Star Online. Retrieved 29 July 2009, from http://thestar.com.my/news

Thibodeau, G. A., \& Patton, K. T. (2002). The human body in health and disease. London: Mosby.

Thrift, A. G., Dewey, H. M., Macdonell, R. A. L., McNeil, J. J., \& Donnan, G. A. (2001). Incidence of the major stroke subtypes. Initial findings from the north east incidence study (NEMESIS). Stroke, 32(8), 1732-1738.

Tilley, S. A. (2003). "Challenging" research practice: Turning a critical lens on the work of transcription. Qualitative Inquiry, 9(5), 750-773.

Twin, S. (1998). An analysis of the effectiveness of focus group as a method of qualitative data collection with Chinese populations in nursing research. Journal of Advanced Nursing, 28(3), 654-661.

Tyson, S. F., Hanley, M., Chillala, J., Selley, A., \& Tallis, C. (2006). Balance disability after stroke. Physical Therapy, 86(1), 30-38.

Van der Gaag, A., Smith, L., Davis, S., Moss, B., Cornelius, V., Laing, S., et al. (2005). Therapy and support services for people with long-term stroke and aphasia and their relatives: A six-month follow-up study. Clinical Rehabilitation, 19(4), 372380.

Vanco, J. D., \& Chan, A. (1994). Living arrangements of older Malaysians: Who coresides with their adult children? Demography, 31(1), 95-113.

Venketsubramaniam, V. (1998). Stroke in Singapore - An overview. Retrieved 20 October, 2005: http://www.sma.org.sg/smj/4001/articles/4001ra1.html

Wachters-Kaufmann, C., Schuling, J., The, H., \& Meyboom-de Jong, B. (2005). Actual and desired information provision after a stroke. Patient Education and Counseling, 56(2), 211-217.

Wagner, E. H. (1998). Chronic disease management: What will it take to improve care for chronic illness? Effective Clinical Practice, 1, 2-4.

Wagner, E. H., Austin, B. T., Davis, C., \& Hindmarsh, M. (2001). Improving chronic illness care: Translating evidence into action. Health Affairs, 20(6), 64-78.

Wagner, E. H., Austin, B. T., \& Von Korff, M. (1996). Organizing care for patients with chronic illness. The Milbank Quarterly, 74(4), 511-544.

Wagner, E. H., Davis, C., Schaefer, J., Korff, M. V., \& Austin, B. (1999). A survey of leading chronic disease management programs: Are they consistent with the literature. Managed Care Quarterly, 7(3), 56-66.

Walling, A. D. (2005). Caregiver training and outcomes in stroke patients. Family Physician, 71(5), 990-992.

Warren, C. A. B. (2002). Qualitative interviewing. In J. F. Gubrium \& J. A. Holstein (Eds.), Handbook of Interview research. Context and method (pp. 83-101). California: Sage Publications. 
Wellwood, I., Dennis, M. S., \& Warlow, C. P. (1994). Perceptions and knowledge of stroke among surviving patients with stroke and their carers. Age ageing, 23(4), 293-298.

Wiles, R., Pain, H., Buckland, S., \& McLellan, L. (1998). Providing appropriate information to patients and carers following a stroke. Journal of Advanced Nursing, 28(4), 794-801.

William, L. S. (1997). Stroke patients' knowledge of stroke: Influence on time to presentation. Stroke, 28(5), 912-915.

Williams, J. (2005). Advances in prevention and treatment of stroke and TIA. Nursing Times, 101(14), 30-32.

Winkens, L. (2006). Manifestations of mental slowness in the daily life of patients with stroke: A qualitative study. Clinical Rehabilitation, 20(9), 827-834.

Wong, K. S. (1999). Risk factors for early death in acute ischemic stroke and intracerebral hemorrhage. A prospective hospital-based study in Asia. Stroke, 30(5), 1065-1069.

World Health Organisation (2003). The World Health Report 2003: Shaping the Future. Geneva: Author.

Yim, J. P. G. (2005). Stroke rehab in Southeast Asia. Rehab Management International, 11(1), 40-41.

Yoon, S. S., \& Byles, J. (2002). Perceptions of stroke in the general public and patients with stroke: A qualitative study. British Medical Journal, 324(7345), 1065-1069.

Young, J., \& Forster, A. (2007). Rehabilitation after stroke. British Medical Journal, 334(7584), 86-90.

Zhu, H. F., Newcommon, N. N., Cooper, M. E., Green, T. L., Seal, B., Klein, G., et al. (2009). Impact of a Stroke Unit on length of hospital stay and in-hospital case fatality. Stroke, 40(I), 18-23.

Zwygart-Stauffacher, M., Lindquist, R., \& Savik, K. (2000). Development of health care delivery systems that are sensitive to the needs of stroke survivors and their caregivers. Nursing Administration Quarterly, 24(3), 33-42. 